\title{
Trojan Horses in the Marine Realm: Characterizing Protistan Parasite Ecology in Coastal Waters
}

\author{
By \\ Taylor Rae Sehein \\ B.S., Northeastern University, 2013 \\ Submitted to the Department of Biology \\ in partial fulfillment of the requirements for the degree of \\ Doctor of Philosophy \\ at the \\ MASSACHUSETTS INSTITUTE OF TECHNOLOGY \\ and the \\ WOODS HOLE OCEANOGRAPHIC INSTITUTION \\ February 2022
}

C2022 Taylor Rae Sehein. All rights reserved.

The author hereby grants to MIT and WHOI permission to reproduce and to distribute publicly paper and electronic copies of this thesis document

in whole or in part in any medium now known or hereafter created.

Author

Joint Program in Biological Oceanography Massachusetts Institute of Technology and Woods Hole Oceanographic Institution

December 14, 2021

Certified by

Dr. Virginia P. Edgcomb

Thesis Supervisor

Accepted by

Woods Hole Oceanographic Institution

Dr. Michael J. Follows

Chair, Joint Committee for Biological Oceanography Massachusetts Institute of Technology/

Woods Hole Oceanographic Institution 


\section{Trojan Horses in the Marine Realm: Characterizing Protistan Parasite Ecology in Coastal Waters}

By

Taylor Rae Sehein

Submitted to the Department of Biology
Massachusetts Institute of Technology
and

Woods Hole Oceanographic Institution

On November 30, 2021 in partial fulfillment of the requirements for the degree of Doctor of Philosophy in the field of Biological Oceanography

Protists are taxonomically and metabolically diverse drivers of energy and nutrient flow in the marine environment, with recent research suggesting significant roles in global carbon cycling throughout the water column. Top-down controls on planktonic protists include grazing and parasitism, processes that both contribute to nutrient transfer and biogeochemical cycling in the global ocean. Recent global surveys of eukaryotic small subunit ribosomal RNA molecular signatures have highlighted the fact that parasites belonging to the marine alveolate order Syndiniales are both abundant and ubiquitous in coastal and open ocean environments, suggesting a major role for this taxon in marine food webs. Two coastal sites, Saanich Inlet (Vancouver Island, BC) and Salt Pond (Falmouth, MA, USA) were selected as model ecosystems to examine the impacts of Syndinian parasitism on protist communities. Data presented in this thesis combines high-resolution sampling, water chemistry (including nutrients) analyses, molecular marker gene analyses, fluorescence in situ hybridization, and modeling to address key knowledge gaps regarding syndinian ecology. Information is presented on previously undescribed putative host taxa, the prevalence of syndinian parasites and infections on different hosts in coastal waters, and a framework for modeling host-parasite interactions based on field observations. 


\section{ACKNOWLEDGEMENTS}

There are many people and institutions to thank for the opportunity to pursue my research interests in this dissertation. First, thank you to the Massachusetts Institute of Technology and Woods Hole Oceanographic Institution for providing a supporting learning environment and funds to share my work at national and international conferences. Research was supported by the WHOI Ocean Venture Fund, the National Science Foundation Biological Oceanography OCE1851012, and the National Science Foundation Graduate Research Fellowship under Grant No. 1745302. Thank you to all the collaborators that lent their expertise to analyze and interpret the wealth of data presented here.

Thank you to the members of my thesis committee for their unwavering support throughout this experience. I am grateful to my advisor, Ginny Edgcomb, for welcoming me into her lab and helping me to navigate through the ups and downs of a PhD. She has always fostered my research interests and offered thoughtful advice both personally and professionally.

Thank you, Becky Gast for sharing your expertise in protist ecology, for supporting field work both in Salt Pond and MVCO, and for assisting in the endless microscopy work. I am thankful for the mentorship of Maria Pachiadaki and her guidance in bioinformatics to analyze large DNA datasets. Thank you to Laure Guillou, who's fundamental research studying syndinian parasites inspired this dissertation. I appreciate the warm welcome I received in Roscoff and the training in CARD-FISH techniques that were essential for this thesis. Last, thank you, Mick Follows, for your enthusiasm for this research and your expertise modeling plankton dynamics and carbon cycling.

Thank you to my dad, mom, and Gia, who always supported my pursuit of a career in science and encouraged me to keep going in tough times. You all now get to refer to me as "Dr. T-Dog," so I guess it was all worth it :)

I am also humbled by all the support from family and friends. Thank you for your love, kindness, and companionship that helped maintain the precarious work-life balance throughout my $\mathrm{PhD}$. 


\section{Table of Contents}

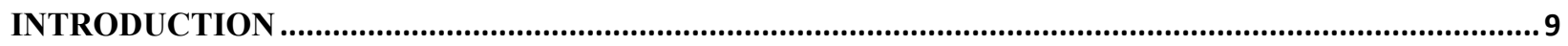

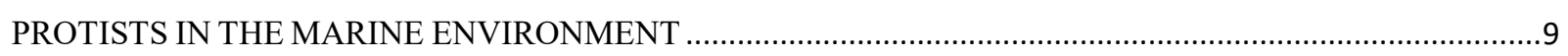

THE LIFESTYLES AND UNIQUE GENOMIC FEATURES OF DINOFLAGELLATES .................................11

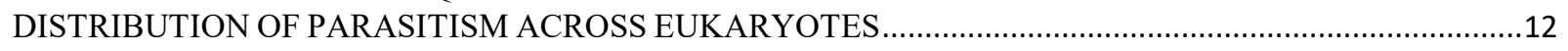

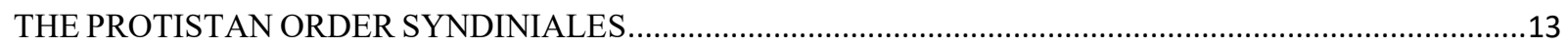

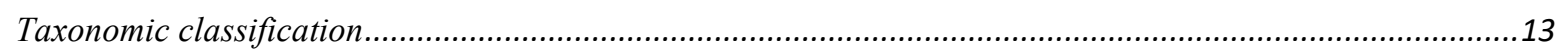

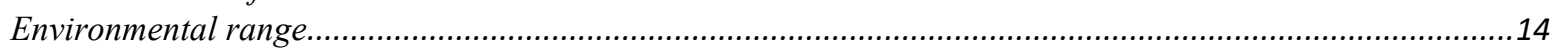

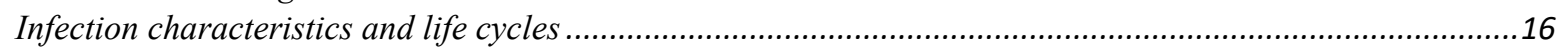

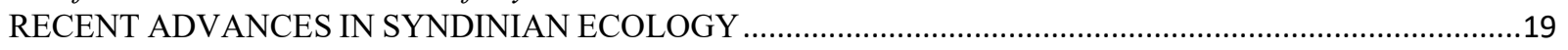

Correlation networks infer host taxa and food web interactions ................................................................19

Epifluorescent microscopy with catalyzed reporter deposition fluorescence in-situ hybridization (CARD-

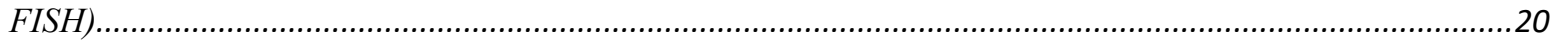

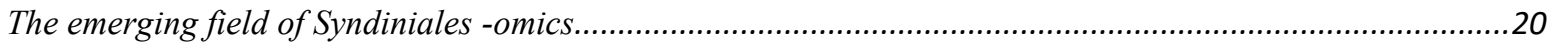

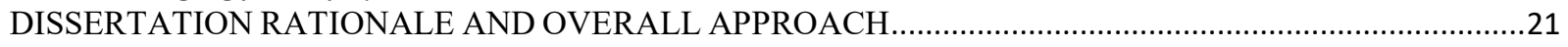

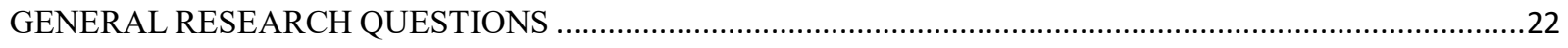

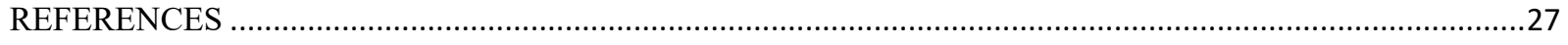

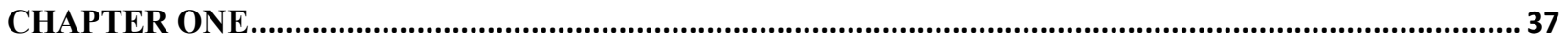

ORIGINALLy PUBLISHED IN DEEP SEA RESEARCH PART II: TOPICAL STUDIES IN OCEANOGRAPHY ..........................37

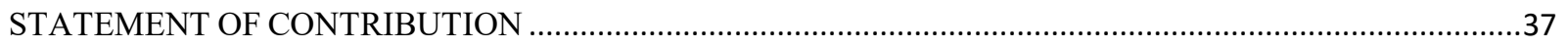

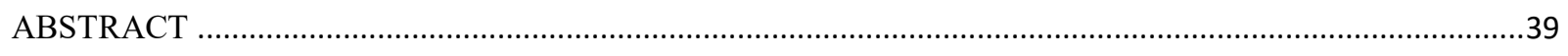

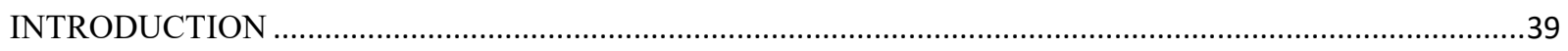

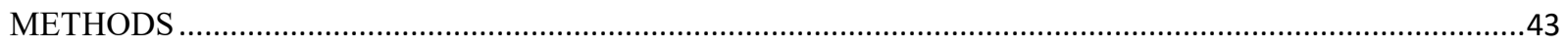

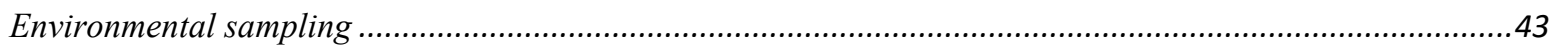

Nucleic acid sampling and extraction .....................................................................................................4

Small subunit ribosomal RNA and RNA gene sequencing and analysis ..................................................45

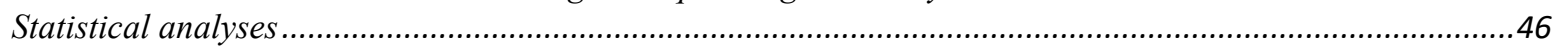

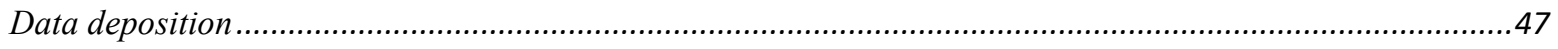

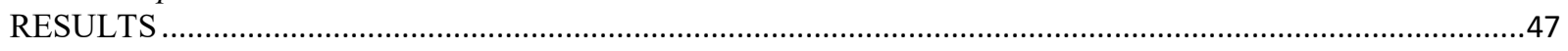

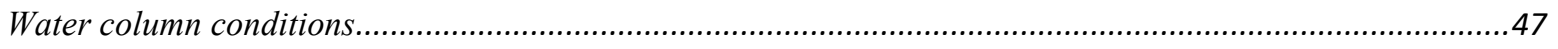

Eukaryotic community structure ……............................................................................................... 48

Exploring protistan co-occurrence patterns ……............................................................................... 49

Insight into potential Syndiniales parasitic interactions ...........................................................................

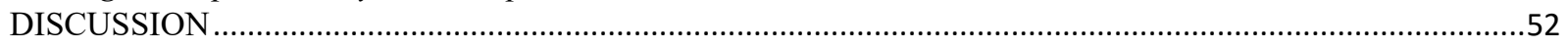

Syndiniales interactions and the significance of peak stratification ……....................................................5

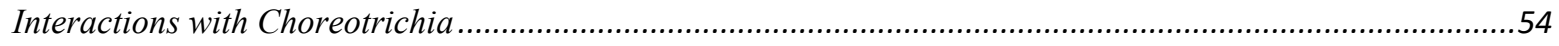

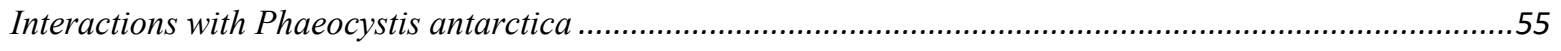

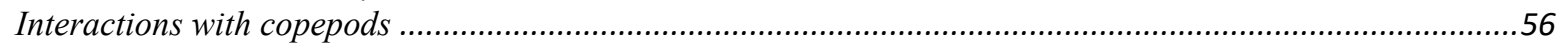

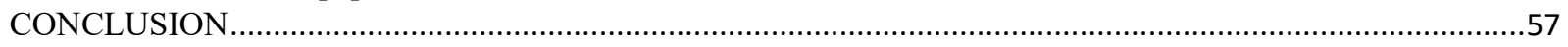

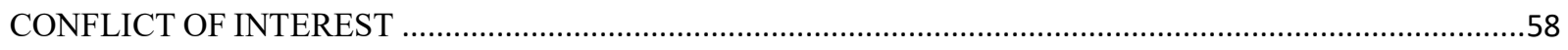

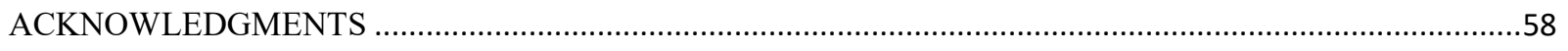

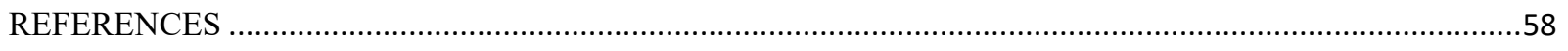

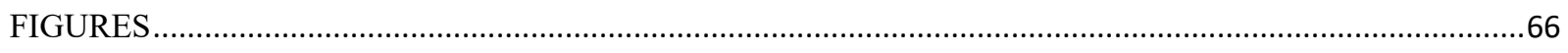

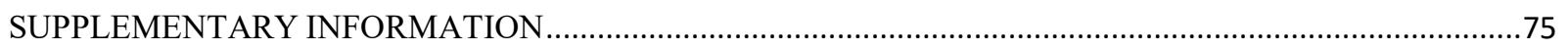

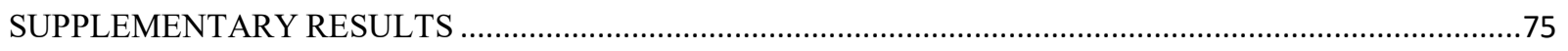

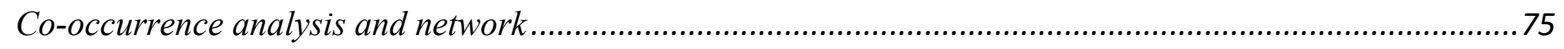

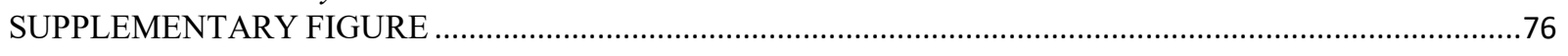

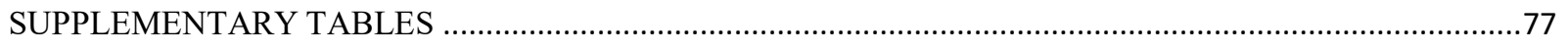

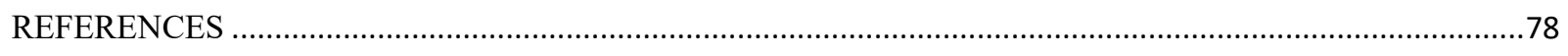

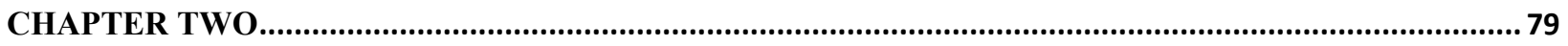




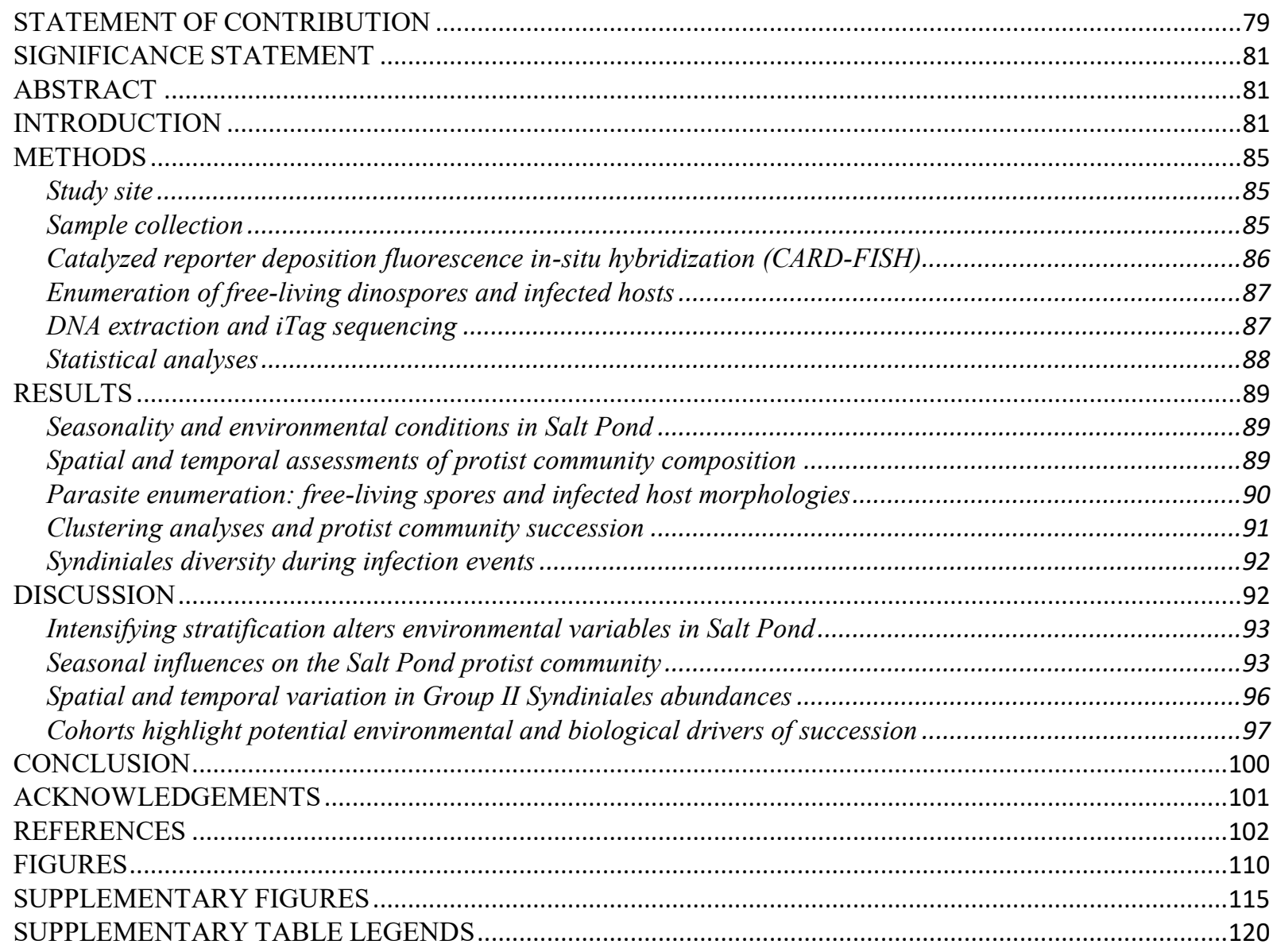

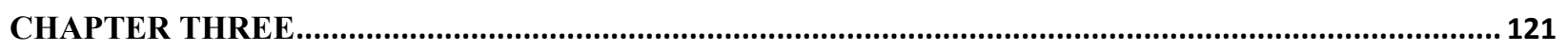

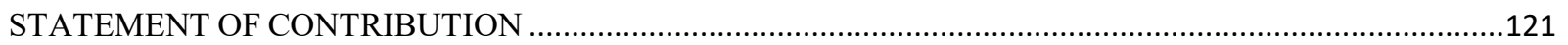

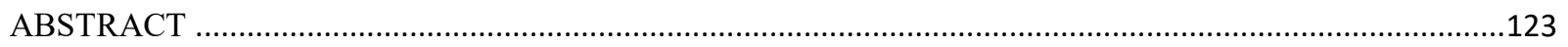

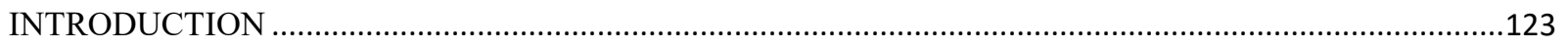

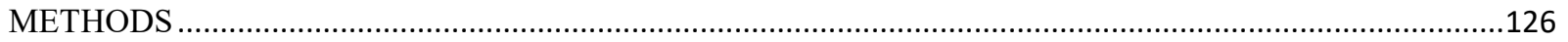

Host-parasite infection experiments and nutrient analyses sample preparation ...........................................126

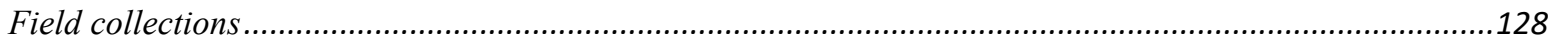

Enumeration of infections and free-living spores using CARD-FISH....................................................128

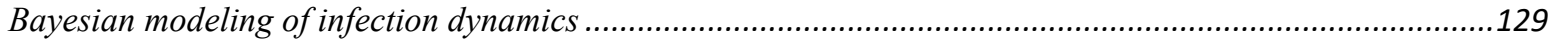

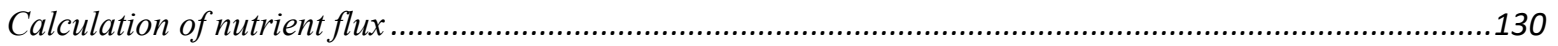

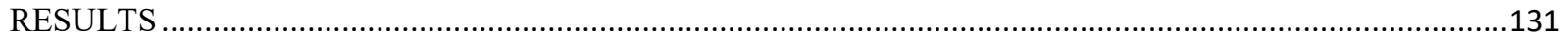

Model performance with host-parasite co-cultures ……...........................................................................131

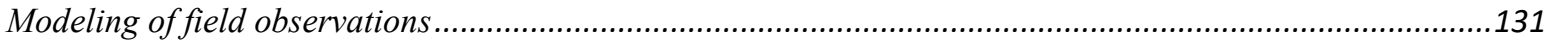

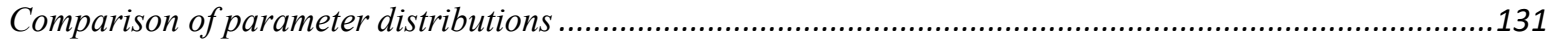

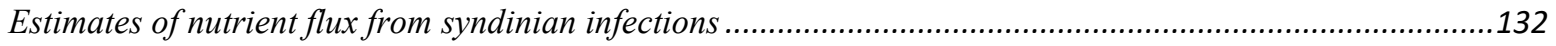

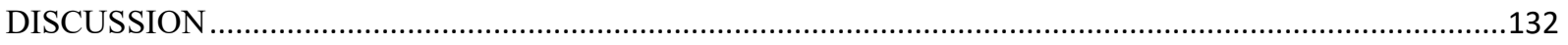

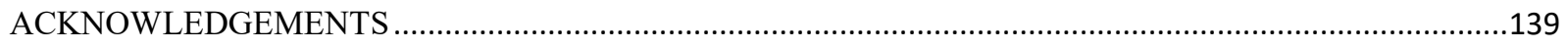

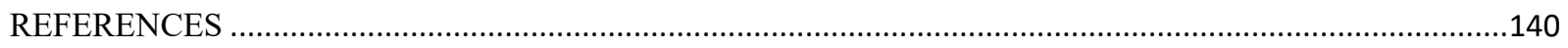

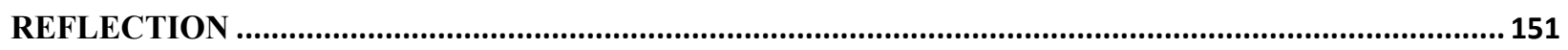

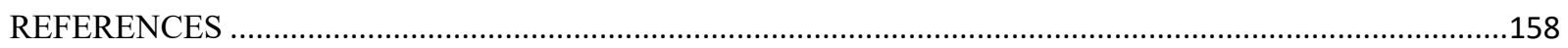




\section{INTRODUCTION}

\section{PROTISTS IN THE MARINE ENVIRONMENT}

The marine carbon cycle is a balancing force that mediates the habitability of the planet. Microbes from the three domains of life (Bacteria, Archaea, Eukarya) play critical roles in carbon fixation, remediation, and sequestration; however, the contributions of microbial eukaryotes to these processes are poorly understood in comparison to the other domains. The microbially-mediated interconversions of carbon species generate essential ecosystem services that impact all trophic levels. Early descriptions of the biological carbon pump recognized select photoautotrophs (diatoms and cocolithophores) as keystone species for the health of marine fisheries and exporters of carbon to the deep ocean (Field et al., 1998, Armbrust, 2009, Higgins et al., 2012, Guidi et al., 2016). However, the field of marine microbial ecology has expanded rapidly in recent decades, revealing much about the diverse metabolic functions and lifestyle of protists that significantly influence marine food webs (Pomeroy, 1974) and biogeochemistry (Azam et al., 1983) in marine systems.

The proportion of carbon sequestered in the deep ocean is impacted by biological interactions that modulate the partitioning between the particulate and dissolved fractions throughout the water column. Sloppy feeding by metazoan grazers (Lampert, 1978, Møller et al., 2003), phytoplankton exudates (Fogg, 1983, Biddanda and Benner, 1997, Biersmith and Benner, 1998), symbioses (including parasitism; Kagami et al., 2007, Jephcott et al., 2016, Scholz et al., 2016) and viral lysis (Middelboe and Lyck, 2002, Breitbart, 2012, Zhao et al., 2019, Kuhlisch et al., 2021) contribute to pools of particulate and dissolved organic matter. The release of carbon from these activities is incorporated into the biomass of heterotrophic bacteria and archaea and transferred up the food chain to small and large flagellates, ciliates, small metazoans, and ultimately, higher tropic levels in a cycle termed the "microbial loop" (Azam et al., 1983, Jumars et al., 1989, Falkowski et al., 1998).

Tracing carbon transformation through biological interactions is essential to understand the magnitude of different contributions to the carbon pump and to evaluate changes that may result from environmental forcing, such as climate change (Beardall et al., 2009, Boyd et al., 2013, Riebesell et al., 2009). Quantifying the uptake and remineralization of carbon and other essential nutrients in-situ remains an area of active research. Early studies in the sunlit ocean 
estimated up to $100 \%$ of primary production is consumed daily by phagotrophic protists, highlighting a major source of mortality for autotrophic and heterotrophic bacteria, as well as phytoplanktonic protists (Verity et al., 1993, Sherr and Sherr, 1994, Tamigneaux et al., 1997, Sherr and Sherr, 2002, Sherr and Sherr, 2009). Advances in in-situ instrumentation have changed the ways in which protist grazing activities are studied by allowing collection of water samples from the environment and execution of grazing studies in situ, thus minimizing the potential artifacts introduced by recovering samples to the sea surface which can expose microorganisms to more significant physico-chemical changes in their environment. These new in situ approaches paired with use of fluorescently-labeled prey (FIP) from the habitat were used to assess protist communities in meso- and bathypelagic habitats (Pachiadaki et al. 2016).

Chemoclines in deep-water masses were found to support enhanced growth of prokaryotes and elevated protist phagotrophy, suggesting these habitats may be important for carbon cycling at depth (Pachiadaki et al., 2016). In a recent study of hydrothermal vent fluids, heterotrophic grazing rates translated to turnover of approximately 1.45-3.77 ug of carbon $\mathrm{L}^{-1} \mathrm{day}^{-1}(\mathrm{Hu}$ et al., 2019). Collectively, such studies have concluded protistan grazing is a significant source of mortality across marine systems that likely influences the diversity and composition of microbial communities; therefore, quantifying grazing and other forms of carbon transformation across a variety of ecosystems remains crucial to our understanding of marine biogeochemical cycles.

Protists contribute to the cycling of other critical nutrients from the air-sea interface to the ocean bottom. Upwelling regions rich in nitrogen and phosphorus stimulate the growth of phytoplanktonic protists, which form the base of food webs for economically-important fisheries (Kudela et al., 2008, Thomas et al., 2009, Silva et al., 2009, Du and Peterson, 2014). Blooms of the marine prymnesiophyte Phaeocystis produce the algal osmolyte dimethylsulfoniopropionate (DMSP; Stefels and Van Boekel, 1993, Keller et al., 1989, van Duyl et al., 1998) and dimethylsulfide (DMS; Stefels et al., 1995, Turner et al., 1988), a volatile compound involved in the global sulfur cycle (Lovelock et al., 1972). DMS in the atmosphere can influence the acidity of rain and the climate by providing a source of nuclei for cloud condensation (Bates et al., 1987, Charlson et al., 1987, Lawrence, 1993). Contributions of protists to nutrient cycling can also be through the activities of their symbionts. In low-oxygen and anoxic environments, symbioses between protists and prokaryotes are common (Bernhard et al., 2000, Edgcomb, 2016). Many prokaryotic endosymbionts of ciliates, flagellates, amoebae, and foraminifera utilize 
chemosynthetic pathways including denitrification, sulfur oxidation, sulfate reduction, and methanogenesis to produce energy (Beinart et al., 2018), thereby connecting the host holobiome to marine nitrogen, sulfur, and methane cycles.

THE LIFESTYLES AND UNIQUE GENOMIC FEATURES OF DINOFLAGELLATES Marine alveolates represent approximately $42 \%$ of all assignable marker gene reads in global surveys of the surface sunlit waters (de Vargas et al., 2015). This supergroup consists mostly of parasitic protists, phototrophic and phagotrophic dinoflagellates, and ciliates, suggesting a significant role for these metabolic strategies across the global ocean (Smetacek, 2001). Dinoflagellates are characterized as taxonomically-diverse, genomically unique, and metabolically flexible, making them highly adaptive to diverse environments (Murray et al., 2016). The ecological success of dinoflagellates can be attributed to several trophic strategies, including alternation between carbon fixation and mixotrophic feeding on organic carbon sources (Bockstahler and Coats, 1993, Stoecker, 1999, Glibert et al., 2008, Mitra et al., 2016), formation of symbiotic relationships (Guillou et al., 2008, Stat et al., 2008), utilization of both planktonic and benthic ecological niches (Hoppenrath et al., 2014), and the production of toxins that impact interactions with competitors and predators (Selander et al., 2006, Tillmann et al., 2008).

Dinoflagellates are underrepresented in genomic repositories, representing just $0.02 \%$ of all genomes available. This paucity of data translates to a very limited understanding of the genetic basis for the myriad adaptations observed in dinoflagellates. Dinoflagellates can be difficult to grow in axenic cultures, which leads to co-sequencing of eukaryotic and bacterial genomes. Dinoflagellate genomes are also large (0.5-40 times the size of the human haploid genome) and contain unique nuclear and organelle features (Lin, 2011, Wisecaver and Hackett, 2011) that can be difficult to reconstruct with standard bioinformatic pipelines. Some dinoflagellates appear to use traditional stop codons to encode alternative amino acids, challenging previous assumptions of the genetic code (Bayer et al., 2012, Bachvaroff, 2019). Dinoflagellates may have also replaced TATA box promoter regions with TTTT sequences, which were noted in conserved regions of the Symbiodinium kawagutii genome (Lin et al., 2015). With continued research into the basic understanding of dinoflagellate genetics, we will 
continue to learn more about the unusual regulatory mechanisms present in individual taxa and determine the extent of these features across the phylum.

\section{DISTRIBUTION OF PARASITISM ACROSS EUKARYOTES}

Parasitism is ubiquitous, yet poorly understood, in marine systems. Protist parasites span eukaryotic lineages across the tree of life, including chytrids, aphelids, stramenopiles, dinoflagellates, cercozoans and phytomyxids (Raghukumar, 1996, Kuhn et al., 2004, James et al., 2006, Changtangsi et al., 2008, Bachvaroff et al., 2014, Karpov et al., 2014, Neuhauser et al., 2014, Chambouvet et al., 2015). Recent depictions of marine food webs and the biological carbon pump recognize that parasitic infections alter marine environments and microbial communities by controlling their host populations and releasing dissolved and particulate organic matter into the surrounding water column (Worden et al., 2015; Figure 1). Parasitism, therefore, is likely a major biological driver of plankton succession (van Donk, 1989) and continued efforts to characterize the host range, infection dynamics, and biogeography of parasitic taxa are warranted.

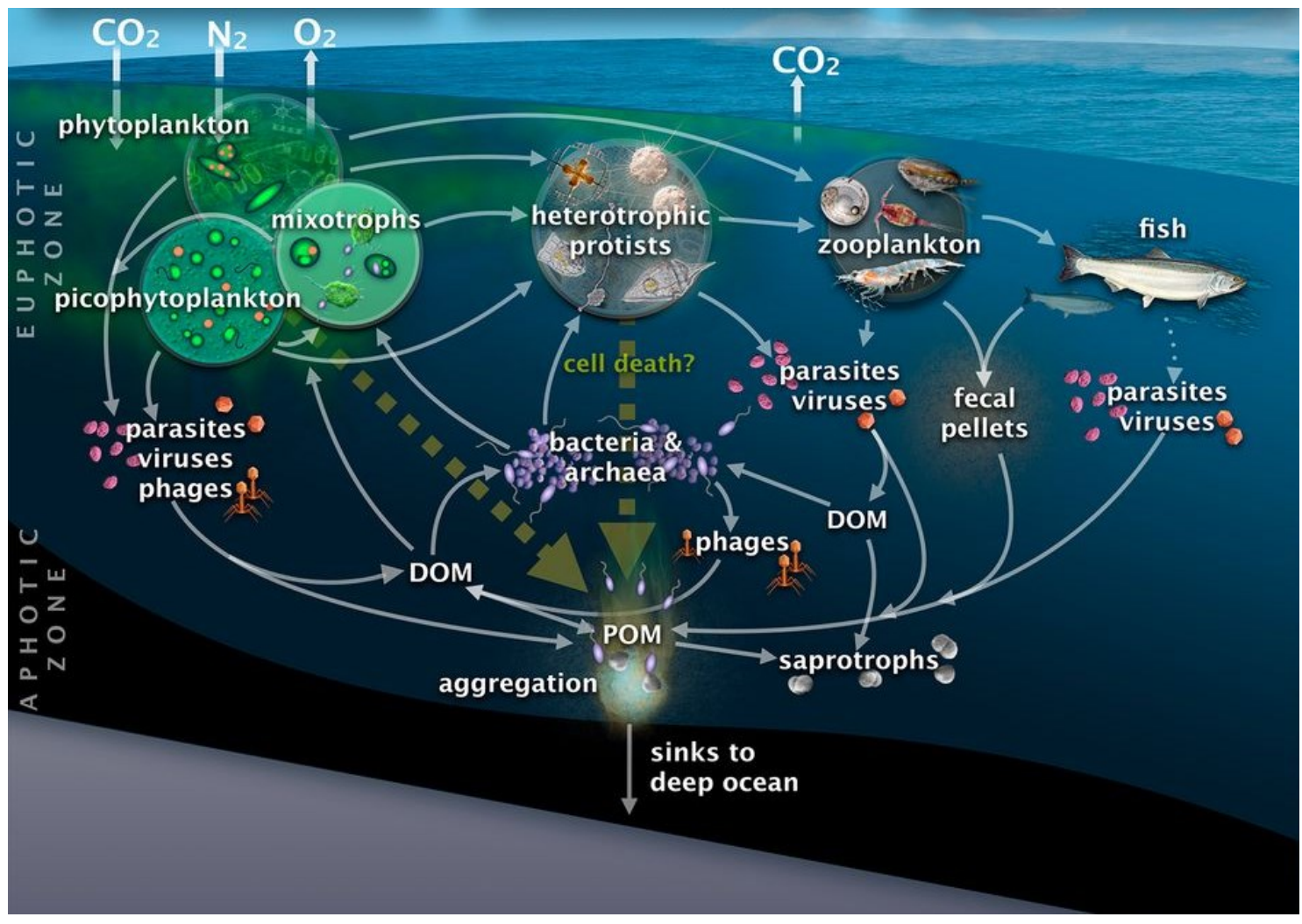

Figure 1. A marine food web depicting various trophic levels and their contributions to pools of particulate and dissolved organic matter in the water column (Worden et al., 2015). 


\section{THE PROTISTAN ORDER SYNDINIALES}

Taxonomic classification

Syndiniales represent a highly diverse order of protists, basal to the phylum Dinoflagellata

(Guillou et al., 2008). To date, few lineages have been formally described, but all known species

have demonstrated a parasitic lifestyle. The order Syndiniales is divided into five distinct taxonomic groups based on the phylogenies revealed by marker gene sequences (Guillou et al., 2008; Figure 2). Sequences from environmental datasets are most commonly annotated to Groups I and II, which can be further assigned to over 100 clades (Guillou et al., 2008. Cai et al., 2020). Limited studies of individual syndinian strains in culture have found clade and sub-clade variability can correspond to generalist and specialist host strategies (Cai et al., 2020). Recent expansion of environmental sequence data in public databases suggests one of the few described syndinian genera, Amoebophrya, spans multiple clades of Group II Syndiniales and includes unique strains that infect different dinoflagellate hosts in culture studies (Coats et al., 1996, Guillou et al., 2008, Kim et al., 2008). This suggests a taxonomic revision is needed to reflect the phylogenetic and ecological diversity of the genus. Groups III, IV and V are often less abundant and diverse in marker gene libraries (de Vargas et al., 2015); however, their distribution across varied marine environments suggests these taxa may also exert parasitic pressure on the surrounding protist and Metazoan populations (Skovgaard et al., 2005, Stentiford and Shields, 2005, Chambouvet et al., 2008). 


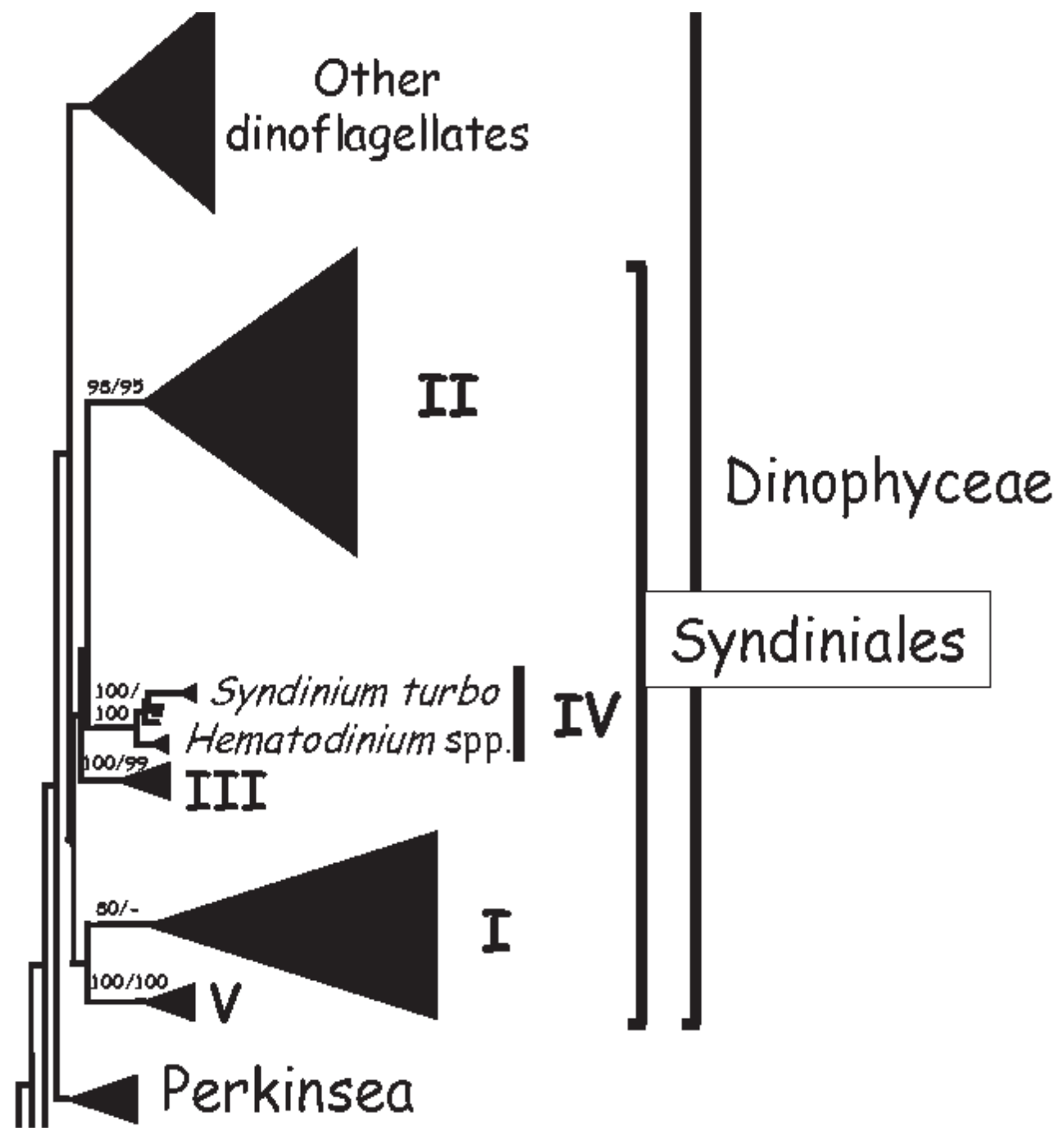

Figure 2. A phylogenetic tree depicting the five groups within the order Syndiniales, positioned basal to the dinoflagellates (Guillou et al., 2008).

Environmental range

Advances in next generation sequencing technologies led to the generation of global ocean marker gene datasets and revealed syndinian parasite sequences are highly abundant in protist small subunit ribosomal RNA (SSU rRNA) marker gene libraries. Tara Oceans surveyed the sunlight surface and mesopelagic waters and found parasitic protist groups accounted for up to $59 \%$ of total heterotrophic protist richness and $53 \%$ of sequence abundance. Among these parasitic sequences, $89 \%$ of picoplankton diversity and abundance were affiliated with Syndiniales Groups I and II (Pawlowski et al., 2012, de Vargas et al., 2015; Figure 3, bubble map). The Malaspina-2010 Expedition found that four protist groups accounted for $69.6 \%$ of sequencing tags in the bathypelagic global ocean, and Group II Syndiniales sequences were the 
most highly represented and phylogenetically diverse group in most samples (Pernice et al., 2015; Figure 3, bubble map). Similar results have been obtained in other environmental surveys of polar regions and oxygen-minimum zones (Cleary and Durbin, 2016, Orsi et al., 2012, Duret et al., 2015, Torres-Beltran et al., 2018). Collectively, surveys of SSU rRNA gene signatures suggest Syndiniales are adapted to both coastal and open ocean environments and likely play a major role in those marine food webs (e.g. Lopez-Garcia et al., 2001, Massana et al., 2004, Not et al., 2007, Edgcomb et al., 2011., Pernice et al., 2015., de Vargas et al., 2015, Christaki et al., 2017., Anderson and Harvey et al., 2020). However, marker gene surveys cannot resolve the extent of parasitic infections in these environments based on abundance of sequences in marker gene datasets because some protist groups, including dinoflagellates, are known to have high SSU rRNA gene copy numbers (Not et al., 2009, Galluzzi et al., 2010, Lin et al., 2011, Pernice et al., 2013), and this may lead to overestimation of their relative abundance in marker gene libraries.

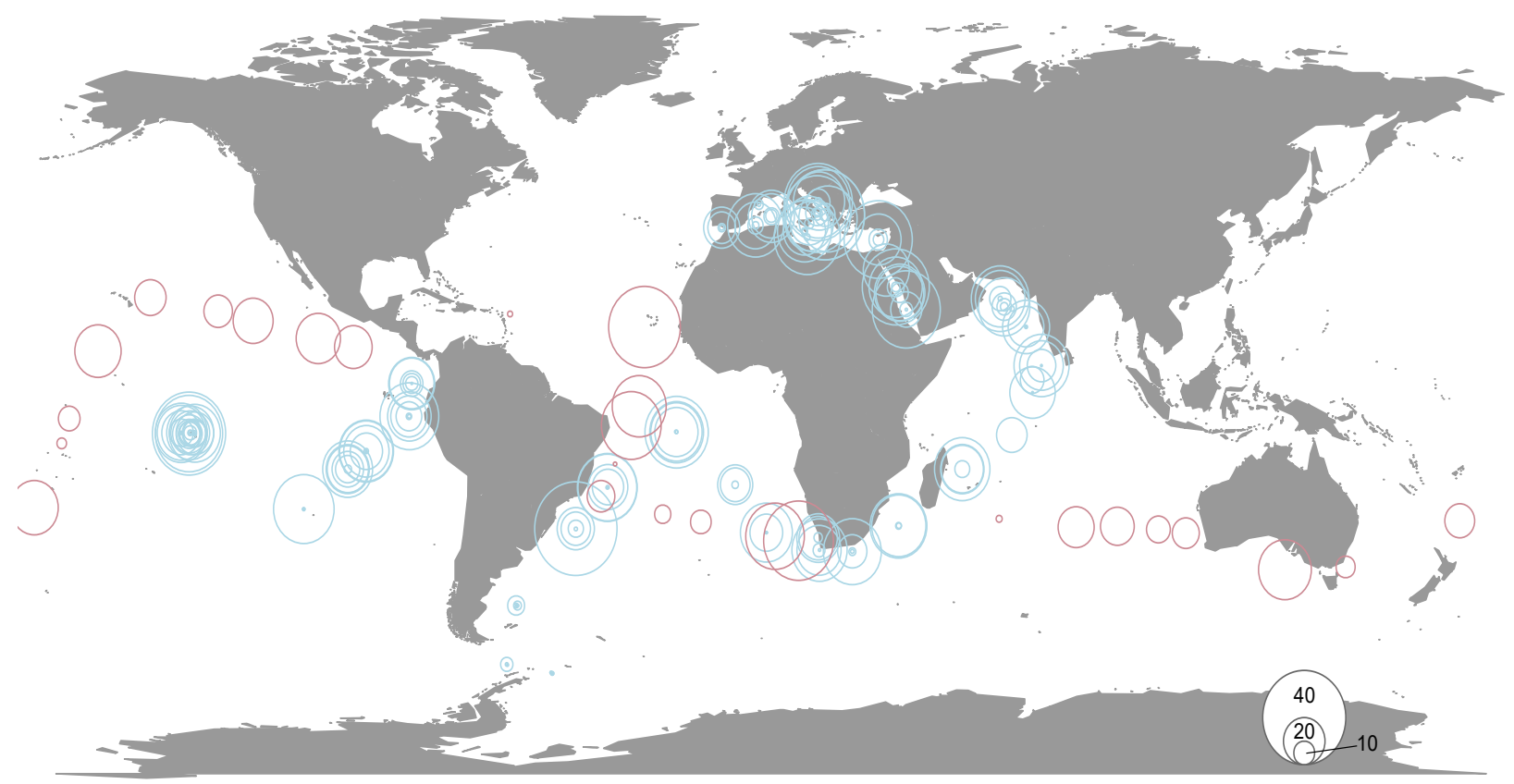

Figure 3. A summary of the relative abundance of Syndiniales sequences observed in samples collected from the sunlight (Tara Oceans, blue) and bathypelagic (Malaspina-2010, red) global ocean surveys. The size of bubble corresponds to the percent of the sequence reads annotated to Syndiniales for the marker gene library prepared at each station. Image credit: Maria Pachiadaki 
Infection characteristics and life cycles

The infection cycles of parasitic Syndiniales clades are described for a limited number of cultured isolates. Host ranges, spore production, infection duration and other variables differ between phylogenetically-defined groups and clades. A recent study found variations in infection strategies even occurred at the sub-clade level, with several individual Group II-Clade 2 parasite cultures found to infect different dinoflagellate species (Cai et al., 2020). It is likely the aforementioned infection properties correspond to broader ecological impacts of syndinian parasitism and, therefore, deserve further study. Given the number of uncultivated and cryptic clades and sub-clades, culture- and marker gene independent strategies appear necessary to determine infection cycle characteristics and intraspecies variability.

Most culture-based research also fails to capture long-lived resting stages of the parasite that likely allow individual strains to persist in the environment in the absence of available host taxa. Chambouvet et al. (2011) described active infections in Scrippsiella trochoidea resting cysts capable of germinating concurrently with host cells; however, there remains a paucity of information on dormant stages in environmental samples. It is possible sediments are a reservoir for resting propagules that emerge when water conditions and host concentrations are favorable. Research characterizing syndinian infections must continue to explore alternative life strategies that may help explain the abundance and ubiquity of Syndiniales parasites across the global ocean.

Among the taxonomically-defined groups, Group I contains eight clades and includes the species Ichthyodinium chaberladi (Group I-Clade 3), a parasitoid of fish eggs, and the genus Euduboscquella (Group I-Clade 4; Guillou et al., 2008). The infection cycle of Euduboscquella is well-studied and has provided some insight into parasite reproductive strategy of this group. The Group I parasites were first described as intracellular spores within a tintinnid ciliate (Duboscq and Collin, 1910, Chatton, 1920). Based on morphological characteristic, the host range was expanded to include aloricate ciliates and the dinoflagellates Noctiluca miliaris and Leptodiscus medusoides (Cachon 1964). Infective spores are bi-flagellated, sigmoid-shaped, 7-10 um long and contained prominent spherical nuclei measuring 4-5 um in diameter. Once initiated, infections develop in the host cytoplasm, forming mature trophonts that measure 60-70 um. Trophonts undergo sporogenesis, creating hundreds of new propagules that are released into the surrounding water column. Infections result in one of three spore types: male, female, and 
neutered. Neutered spores do not interact with male or female spores, but cells contain vacuoles and may facilitate extended survival outside of a host. Male spores are flagellated and swim actively, but quickly lose motility and degenerate in one to two days. Female spores are larger, spherical, contained ovoid nuclei and survive 2-3 days. Male and female spores can fuse and perform two successive divisions. The resulting spores resemble neutered spores (Coats et al., 2012). The production of multiple spore types with differing morphological and behavioral characteristics may indicate mechanisms of sexual reproduction and resting stages that could facilitate long-term parasite survival.

Syndiniales Group II is genetically more diverse than Group I, with over 100 putative clades. Prior to large-scale sequencing efforts, the genus Amoebophrya was formally described within Group II based on phylogenetic comparisons of sequences from cultures and single cells; however, all sequences previously within the monophyletic group Amoebophrya were reassigned to clades 1-5, 25, 33, 41 and 42 (Guillou et al., 2008). Hosts of Amoebophrya parasites include the thecate dinoflagellates Alexandrium sp. (Nishitani et al., 1985, Jacobson, 1987, Sengco et al., 2003), Ceratium tripos (Li et al., 2014), Dinophysis sp. (Fritz and Nass, 1992, Salomon et al., 2003, Salomon et al., 2009), Gonyaulax polygramma (Kim et al., 2008, Siano et al., 2011), Prorocentrum micans/minimum (Maranda, 2001, Salomon et al., 2009), and Scrippsiella sp. (Coats et al., 1996, Chambouvet et al., 2011b, Li et al., 2014), athecate dinoflagellates Akashiwo sanguinea (Coats and Bockstahler, 1994, Coats et al., 1996, Mazzillo et al., 2011) and Karlodinium veneficum (Gunderson et al., 2002), and the radiolarian Hexacontium gigantheum (Bråte et al., 2012). Host type and parasite strain diversity correspond with variations in infection characteristics, but infection cycles share similar properties. Like Group I strains, infective spores are small (8-10 um long) and biflagellate. Infections are initiated when a spore attaches to the surface of the host organism and passes through the host pellicle. Trophonts develop in the cytoplasm or nucleus and reach maturity in 2-3 days, forming a multinucleated structure known as a "beehive." As the beehive structure metamorphoses into an elongated vermiform structure, internal pressure ruptures the host cell membrane and releases the parasite into the surrounding water column. The temporary vermiform, which is comprised of individual spores, then disassociates into dozens to hundreds of new infective spores (Coats and Park, 2002; Figure 4). Microscopic confirmation across clades is needed to determine if these infection stages can be generalized for all Group II Syndiniales. 


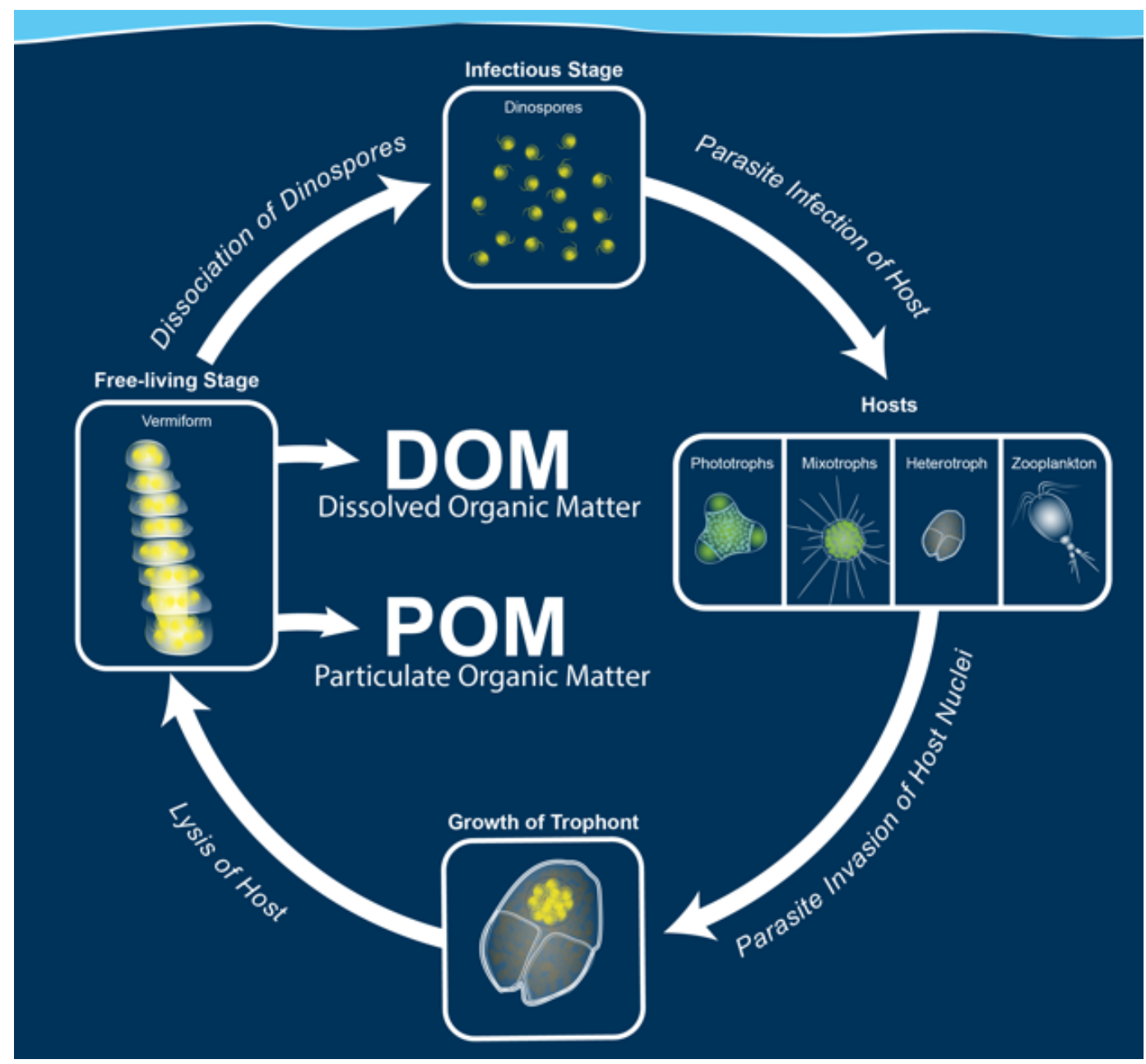

Figure 4. A generalized depiction of the parasitic Syndiniales life cycle. Image credit: WHOI Graphics

Unlike the diverse Groups I and II, organisms annotated to Group IV Syndiniales can be classified as belonging to either the genus Syndinium or the genus Hematodinium and target Metazoan hosts. Syndinium infects copepods, including Paracalanus parvus and Corycaeus sp (Skovgaard et al., 2005). Infections develop within the host body cavity and reach maturity when numerous spores are released from the exoskeleton, killing the host. Descriptions of micro- and macrospores suggest male/female sexes or vegetative/dormant states. Parasites belonging to the genus Hematodinium are important parasites of marine Crustacea (Stentiford and Shields, 2005). Hosts include the commercially-important Norway lobster Nephrops norvegicus, snow crab Chionoecetes opilio, Tanner crab C. bairdi, American blue crab Callinectes sapidus, and the velvet swimming crab Necora puber. Both the morphology and infection stages of Hematodinium differ from other syndinian taxa. Free-living spores develop into filamentous 
trophonts that increase in size and complexity as the parasite grows. Infections are localized to the hemolymph or hepatopancreas, depending on host species. When the infection reaches maturity, spores are formed; however, spores can either be released from the host or regrown into new filamentous colonies (Appleton and Vickerman, 1998). The proliferation of parasite spores drains the host of proteins and carbohydrates. The destruction of critical tissues results in starvation, lethargy, and hypoxia, which contribute to host morbidity (Taylor et al., 1996, Stentiford et al.,2000, 2001, Shields et al., 2003). Although the physiologies of free-living parasites spores are similar across syndinian groups I, II, and IV, the distinct host preferences and infection stages may correlate with the metabolic requirements and nutrient acquisition strategies of individual taxa. Host ranges and infection characteristics for Groups III and V are yet to be determined because taxa belonging to these groups are known only by marker gene sequences obtained from environmental samples.

\section{RECENT ADVANCES IN SYNDINIAN ECOLOGY}

Correlation networks infer host taxa and food web interactions

Marker gene datasets provide snapshots of microbial communities both across diverse habitats and through time. These data can be useful for inferring interactions between protists when direct observations cannot be made. Correlation networks leverage statistical relationships among organisms and environmental variables to hypothesize trophic interactions and other ecological processes. For example, the eastern English Channel is a coastal system characterized by seasonal blooms of the haptophyte Phaeocystis globosa and abundant populations of colonial diatoms and dinoflagellates, including syndinian parasites. A correlation network identified putative interactions between Group II Syndiniales parasites and the dinoflagellates Gyrodinium sp., Warnowia sp., and Lessardia elongate (Christaki et al., 2017). Infections of Gyrodinium by Syndiniales have been documented in Chesapeake Bay (Maryland, USA; Coats et al., 1996), but there are no records of the other two dinoflagellates hosting syndinian parasites. Other connections in the English Channel network analysis indicated associations between Syndiniales and diatoms, ciliates, MAST stramenopiles, and picobiliphytes. Some of these connections may represent interactions other than parasitism, including ciliates grazing on parasite spores or saprotrophy of released dissolved and particulate matter from host cell lysis (Anderson and Harvey, 2020). Other network studies from diverse marine systems documented positive and 
negative associations between syndinian parasites and diatom and ciliate taxa, suggesting interactions between the groups are complex and may be species- and site-specific.

Epifluorescent microscopy with catalyzed reporter deposition fluorescence in-situ hybridization (CARD-FISH)

Visualization of species interactions is important to confirm the hypotheses generated by correlation networks. Catalyzed reporter deposition fluorescence in-situ hybridization (CARDFISH) is a more sensitive approach than light microscopy and has been used to successfully quantify free-living dinospores and infections in coastal, open ocean, and oligotrophic ocean water masses (Chambouvet et al., 2011b, Siano et al., 2011, Velo-Suarez et al., 2013). Fluorescent probes have been designed to target Group II Syndiniales (73 of 125 clades; ALV01) and several individual clades (Chambouvet et al., 2008). Applications of CARD-FISH show that Group II parasite infections increase in abundance during the peak through the termination of some dinoflagellate blooms, including some harmful algal bloom taxa (Coats and Bockstahler, 1994, Coats and Park, 2002, Velo-Suarez et al., 2013). This suggests syndinian parasites may exert top-down pressure on bloom-forming taxa. In the Penzé Estuary (Roscoff, France), up to $46 \%$ of dinoflagellates were infected (average of $21 \%$ ) over a 3-month period during the summer (Chambouvet et al., 2008). CARD-FISH probes and epifluorescent microscopy can highlight interactions between hosts with distinct morphologies (Siano et al., 2011) and Group II Syndiniales parasites for which clade-specific probes have been designed (Chambouvet et al., 2008).

The emerging field of Syndiniales -omics

Advancements in single-cell sequencing and bioinformatics have expanded the tools available to study the mechanisms of parasitism and overcome challenges associated with the uncultivable microbial majority. Host Alexandrium fundyense gene expression was assessed in the physical presence of Amoebophrya parasites during exposure to filtrate containing waterborne cues from infected cultures (Lu et al., 2016). Both treatments resulted in changes to the host gene expression profiles, including the upregulation of genes associated with energy acquisition that reflected increased demand and signal transduction suggestive of a threat-sensing mechanism (Lu et al., 2016). Farhat et al. (2018) compared the gene expression of two Syndiniales strains, one that exhibited a generalist strategy by infecting multiple hosts and one that specialized in a 
single host taxon. The "generalist" strain expressed a greater variety of genes associated with cellular membrane reconstruction and adhesion protein synthesis that may facilitate interactions with a wider range of host species (Farhat et al., 2018). Another recent study found the host chloroplasts and mitochondria remained functional during the development of an Amoebophrya infection, which may support host fitness as nuclear material is degraded by the parasite (Kayal et al., 2020). It is unlikely these few transcriptome studies are representative of the diverse syndinian order overall; therefore, continued research is needed characterize the molecular underpinning of different infection strategies.

The first assembled syndinian genome, a Group II-Clade 2 strain, revealed that the parasite retained most genes essential for survival, with the exception of genes for the biosynthesis of a few amino acids and isoprenoids (John et al., 2019). Given that loss of genes or function and reduced genome size is common in other symbionts, these findings were unexpected but may reflect the retention of a free-living life stage of these parasites. Although the parasite contained functional mitochondria, essential mitochondrial genes associated with the electron transport system and oxidative phosphorylation had migrated to the parasite nucleus and the mitochondrial genome appeared to be absent, suggesting some evolutionary streamlining had occurred (John et al., 2019). Further characterization of Syndiniales genomes may uncover other interesting features relevant to eukaryotic evolution and ecological roles, including alternative metabolic strategies or pathways that extend life in the free-living stage when susceptible hosts are not prevalent in the water column.

\section{DISSERTATION RATIONALE AND OVERALL APPROACH}

Our fundamental understanding of protist parasitism has been inferred from a limited number of culture-based studies, marker gene surveys, and epifluorescent microscopy observations. These studies, however, often fall short of characterizing hosts for many syndinian clades known only by their marker gene sequences, quantifying the impacts on protist populations, and estimating contributions to pools of particulate and dissolved organic matter. Given the prevalence of syndinian sequences in marker gene datasets across the global ocean and the diverse range of hosts, Syndiniales parasites likely play key roles in marine food webs and biogeochemical cycling. The following research uses marker gene datasets to broadly assess all populations of syndinian parasites and infer putative host populations through correlation analyses. Targeted approaches focus on Group II Syndiniales because of their overall abundance in marine waters 
(Pernice et al., 2013, de Vargas et al., 2015), extensive diversity (Guillou et al., 2008, Cai et al., 2020), and documented impacts on dinoflagellate populations (Chambouvet et al., 2008, Chambouvet et al., 2011b, Siano et al., 2011), which are one of the most important planktonic groups (Zehr and Voytek, 2012). We aim to address persisting knowledge gaps in syndinian ecology by combining high-resolution sampling, water chemistry analyses, molecular marker gene analyses, fluorescence microscopy, culture-based studies, and modeling to produce the first focused assessment of host-Group II Syndiniales dynamics in coastal marine ecosystems.

\section{GENERAL RESEARCH QUESTIONS}

This dissertation leverages high resolutions datasets from two coastal field sites (Saanich Inlet, Vancouver Island, BC and Salt Pond, Falmouth, MA) to investigate syndinian parasites diversity and the potential trophic impacts of parasitism in marine systems. It utilizes robust DNA datasets to infer putative host-parasite interactions based on statistically significant co-occurrences. Infection prevalence among various protist populations is quantified using CARD-FISH. Last, culture-based experiments are used to quantify key nutrients released through host cell lysis, in addition to Bayesian modeling of host-parasite interactions, to estimate nutrient flux resulting from syndinian infection events.

To evaluate the ecological roles of syndinian parasites in coastal marine ecosystems, the dissertation research had three main goals:

1. Evaluate temporal dynamics of protistan community composition using high resolution sampling to elucidate how host and parasite diversity may shift seasonally, and how this may correlate with environmental variables (nutrients, temperature, salinity, dissolved oxygen, depth).

2. Examine temporal variation in levels of infection of the protist community and hostparasite specificity using high-resolution sampling in Salt Pond to inform on host-parasite dynamics, the impacts of Syndiniales infections on populations of their hosts at this site, and the range of hosts that are susceptible to infection by different Group II Syndiniales clades.

3. Determine the potential impact of infected protists on environmental pools of dissolved and particulate organic matter. 
Chapter 1: Generating hypotheses about putative parasite hosts within complex microbial communities using network analyses

Oxygen minimum zones, particularly in coastal environments, are expanding in response to anthropogenic additions of nutrients and the subsequent redox gradients established by oxygen depletion are considered hot spots for microbial activities. Protists participate in wide-ranging interactions that couple metabolic processes and behavior to nutrient and energy flow in these environments. Parasitism, in particular, may exert top-down control on susceptible host taxa and release dissolved and particulate organic matter back in to the environment, diverting energy away from grazers and higher trophic levels and fueling prokaryotic microbial metabolisms (Azam et al., 1983; Taylor et al., 1986). Saanich Inlet (Vancouver Island, B.C.), a seasonally stratified coastal fjord, was selected as a natural laboratory to examine how ocean deoxygenation shapes protist community dynamics and interactions, with a focus on Syndiniales and putative host taxa.

Previous studies revealed syndinian parasites are prevalent in Saanich Inlet, with molecular signatures representing approximately $90 \%$ of all Dinophyceae in the oxic surface waters across all seasons and about $80 \%$ of sequences at $200 \mathrm{~m}$ during periods of deep water renewal (Orsi et al., 2012). Molecular signatures of Syndiniales at depth could represent active infections, sinking of free-living spores, or transport to depth by water recirculation; however, the prevalence of molecular signatures at the surface suggest Syndiniales are actively infecting protist populations throughout the year. Methods for investigating top-down controls often require an in-depth understanding of microbial interactions that may not be available in understudied environments or for uncultured protist taxa. Co-occurrence networks, however, are powerful hypothesis-generating tools that utilize cost- and time-efficient barcoded DNA libraries generated from environmental samples to infer potential interactions between taxa based on statistically significant co-presence of molecular signatures. In this chapter, co-occurrence networks will be constructed from monthly surveys conducted during the period of peak stratification and deep-water renewal (May-October) in Saanich Inlet. It is hypothesized that, due to the host-dependent life cycle of described Syndiniales parasites, potential host taxa will have statistically- significant patterns of co-presence and absence with syndinian parasite molecular signatures in the water column. Observations of protistan taxa co-occurring with syndinian 
parasites across both spatial and temporal scales will shed light on putative protist hosts and on potential influences of parasitism on pools of organic matter released and on termination of phytoplankton bloom events, both of which have implications for coastal marine food webs and biogeochemical cycling.

Chapter 2: Parasite impacts on the overall protist community and strain-specific host-parasite interactions during periods of peak productivity Syndiniales parasites are suspected to infect a diverse range of hosts and infections may exert significant pressure on susceptible protist populations. While analyses of marker genes, mRNA pools (transcribed genes), and genomic DNA recovered from environmental samples can provide insights into what taxa are present, they do not provide definitive identification of Syndiniales hosts (Torres-Beltran et al. 2018). Microscopic observations of labeled cells can inform on overall infection rates, but it is often difficult obtain more than the general identity of hosts (e.g., phylum level). Molecular probes have been successfully applied to a subgroup of Syndiniales using fluorescence in situ hybridization (FISH) techniques to visualize infections in host cells and to characterize infection rates in selected bloom populations (Chambouvet et al., 2008; Chambouvet et al., 2011b; Velo-Suarez et al., 2013). However, most information on hostparasite specificity has come from studies of a few cultured isolates (e.g., Siano et al., 2011; Chambouvet et al., 2008). One of the best public sources of information, the Aquasymbio.fr website, synthesizes published information on Syndiniales and has information on only 10 out of over 100 clades of MALV Groups I and II (Guillou et al., 2008, Cai et al., 2020). Little information exists on the breadth of hosts for the majority of Syndiniales clades. For a greater understanding of the ecology of Syndiniales and of their impact on dominant protist plankton, significantly more information is required about host populations and host-parasite specificity.

To address these fundamental ecological questions, traditional molecular techniques will be applied to samples obtained from a coastal site (Salt Pond, Falmouth) to assess overall Syndiniales parasitism impacts on the protist community and to identify strain-specific interactions between hosts and parasites. Preliminary surveys of protist diversity and Syndiniales infection rates were conducted weekly in Salt Pond (Falmouth, MA) from July 10 to August 31 in 2017 using molecular methods and microscopy. Five MALV Group I and 22 MALV Group II clade molecular signatures were detected, with diversity varying across sampling dates. The 
relatively coarse sampling frequency of weekly sampling did not allow for estimation of rates of infection or insight into infection dynamics because this frequency was greater than the duration of a typical infection ( $2-3$ days).

These preliminary data suggest Salt Pond is a diverse and dynamic coastal system suitable for the in-depth study of Syndiniales diversity. Salt Pond is proximal to the laboratory at Woods Hole Oceanographic Institution, allowing for more frequent sampling, and thus, examination of host-parasite dynamics. High-resolution data sets will be generated for Salt Pond across periods of peak productivity, where host populations are expected to be most abundant. iTag libraries, quantification of free-living spores and host infections using FISH, and water column chemistry analyses will be used to gain new insights into the seasonal shifts in protist community composition and effects on Group II Syndiniales parasite diversity and infection prevalence.

Chapter 3: Culture-based assessments of carbon and nutrient release for key host taxa and modeling of parasite contributions to biogeochemical cycling in Salt Pond

Current paradigms of marine carbon cycling (e.g., Worden et al. 2015) now recognize and include parasites and viruses in depictions of transfers of carbon between the euphotic zone, aphotic zone, and the benthos, however practically no quantitative information exists on the impact of those marine protist parasites. Host cell lysis from parasitic infection releases key nutrients, like carbon and nitrogen, back in to the water column where they can be used to fuel microbial metabolic processes. Unfortunately, the source of dissolved and particulate nutrients cannot be easily traced back to specific origins in the marine environment, making it difficult to quantify the impacts of parasitism on biogeochemical cycling vs. viral lysis, cell death, and predation. Furthermore, the open ocean, and Salt Pond are open systems, where sources and sinks of nutrients vary continuously, making it impossible to quantify the release of nutrients from any specific individual process. Widespread and abundant molecular signatures of Syndiniales parasites suggest they may exert significant top-down pressures on protist populations throughout the global ocean; however, no quantitative data exist to describe the particulate and dissolved organic matter released by infection events. Salt Pond will be used as a model system to estimate the fraction of nutrients released that can be attributed to Syndiniales parasitism in a local estuarine environment. The following approach will integrate culture-based 
measurements, modeling methods, and environmental sampling. The overall hypothesis is that this parasitic protist group that has heretofore been largely overlooked in models of marine carbon cycling, plays a major role in affecting populations of its hosts and in the release of labile particulate and dissolved organic carbon and nitrogen in marine ecosystems. 


\section{REFERENCES}

Alves-de-Souza, C., Varela, D., Iriarte, J.L., González, H.E. and Guillou, L., 2012. Infection dynamics of Amoebophryidae parasitoids on harmful dinoflagellates in a southern Chilean fjord dominated by diatoms. Aquatic Microbial Ecology, 66(2), pp.183-197.

Anderson, S.R. and Harvey, E.L., 2020. Temporal Variability and Ecological Interactions of Parasitic Marine Syndiniales in Coastal Protist Communities. Msphere 5(3).

Appleton, P.L. and Vickerman, K., 1996. Presence of apicomplexan-type micropores in a parasitic dinoflagellate, Hematodinium sp. Parasitology Research, 82(3), pp.279-282.

Armbrust, E.V., 2009. The life of diatoms in the world's oceans. Nature, 459(7244), pp.185-192.

Azam, F., Fenchel, T., Field, J.G., Gray, J.S., Meyer-Reil, L.A. and Thingstad, F., 1983. The ecological role of water-column microbes in the sea. Marine Ecology Progress Series, pp.257263.

Bachvaroff, T.R., Gornik, S.G., Concepcion, G.T., Waller, R.F., Mendez, G.S., Lippmeier, J.C. and Delwiche, C.F., 2014. Dinoflagellate phylogeny revisited: Using ribosomal proteins to resolve deep branching dinoflagellate clades. Molecular Phylogenetics and Evolution, 70, pp.314-322.

Bachvaroff, T.R., 2019. A precedented nuclear genetic code with all three termination codons reassigned as sense codons in the syndinean Amoebophrya sp. ex Karlodinium veneficum. PloS One, 14(2), p.e0212912.

Bates, T.S., Charlson, R.J. and Gammon, R.H., 1987. Evidence for the climatic role of marine biogenic sulphur. Nature, 329(6137), pp.319-321.

Bayer, T., Aranda, M., Sunagawa, S., Yum, L.K., DeSalvo, M.K., Lindquist, E., Coffroth, M.A., Voolstra, C.R. and Medina, M., 2012. Symbiodinium transcriptomes: genome insights into the dinoflagellate symbionts of reef-building corals. PloS One, 7(4), p.e35269.

Beardall, J., Stojkovic, S. and Larsen, S., 2009. Living in a high CO2 world: impacts of global climate change on marine phytoplankton. Plant Ecology and Diversity, 2(2), pp.191-205.

Beinart, R.A., Rotterová, J., Čepička, I., Gast, R.J. and Edgcomb, V.P., 2018. The genome of an endosymbiotic methanogen is very similar to those of its free-living relatives. Environmental Microbiology, 20(7), pp.2538-2551.

Bernhard, J.M., Buck, K.R., Farmer, M.A. and Bowser, S.S., 2000. The Santa Barbara Basin is a symbiosis oasis. Nature, 403(6765), pp.77-80. 
Biddanda, B. and Benner, R., 1997. Carbon, nitrogen, and carbohydrate fluxes during the production of particulate and dissolved organic matter by marine phytoplankton. Limnology and Oceanography, 42(3), pp.506-518.

Bockstahler, K.R. and Coats, D.W., 1993. Spatial and temporal aspects of mixotrophy in Chesapeake Bay dinoflagellates. Journal of Eukaryotic Microbiology, 40(1), pp.49-60.

Boyd, P.W., Rynearson, T.A., Armstrong, E.A., Fu, F., Hayashi, K., Hu, Z., Hutchins, D.A., Kudela, R.M., Litchman, E., Mulholland, M.R. and Passow, U., 2013. Marine phytoplankton temperature versus growth responses from polar to tropical waters-outcome of a scientific community-wide study. PloS One, 8(5), p.e63091.

Bråte, J., Krabberød, A. K., Dolven, J. K., Ose, R. F., Kristensen, T., Bjørklund, K. R., Shalchian-Tabrizi, K., 2012. Radiolaria associated with large diversity of marine alveolates. Protist, 163, pp.767-777.

Breitbart, M., 2012. Marine viruses: truth or dare. Annual Review of Marine Science, 4, pp.425448.

Cachon, J., 1964. Contribution à l'étude des péridiniens parasites. Cytologie, cycles évolutifs. Annales des sciences naturalles zoologie, 6, pp.1-158.

Chambouvet, A., Morin, P., Marie, D., and Guillou, L., 2008. Control of toxic marine dinoflagellate blooms by serial parasitic killers. Science, 322, pp.1254-1257.

Chambouvet, A., Alves-de-Souza, C., Cueff, V., Marie, D., Karpov, S., and Guillou, L., 2011. Interplay between the parasite Amoebophrya sp. (Alveolata) and the cyst formation of the red tide dinoflagellate Scrippsiella trochoidea. Protist, 162(4), pp.637-649.

Chambouvet, A., Laabir, M., Sengco, M., Vaquer, A., and Guillou, L., 2011b. Genetic diversity of Amoebophryidae (Syndiniales) during Alexandrium catenella/tamarense (Dinophyceae) blooms in the Thau lagoon (Mediterranean Sea, France). Research in Microbiology, 162, pp.959968.

Chambouvet, A., Richards, T.A., Bass, D., Neuhauser, S., 2015. Parasite Diversity and Diversification: Evolutionary Ecology Meets Phylogenetics. Cambridge University Press; Revealing Microparasite Diversity in Aquatic Environments Using Brute Force Molecular Techniques and Subtle Microscopy; p. 93

Chantangsi, C., Esson, H.J. and Leander, B.S., 2008. Morphology and molecular phylogeny of a marine interstitial tetraflagellate with putative endosymbionts: Auranticordis quadriverberis n. gen. et sp. (Cercozoa). BMC Microbiology, 8(1), pp.1-16.

Charlson, R.J., Lovelock, J.E., Andreae, M.O. and Warren, S.G., 1987. Oceanic phytoplankton, atmospheric sulphur, cloud albedo and climate. Nature, 326(6114), pp.655-661. 
Chatton, É., 1920. Les peridiniens parasites: morphologie, reproduction, ethologie. Archives of Zoological Experimental Genetics, 59, pp.1-475.

Christaki, U., Genitsaris, S., Monchy, S., Li, L.L., Rachik, S., Breton, E. and Sime-Ngando, T., 2017. Parasitic eukaryotes in a meso-eutrophic coastal system with marked Phaeocystis globosa blooms. Frontiers in Marine Science, 4, pp.416.

Coats, D.W. and Bockstahler, K.R., 1994. Amoebophrya ceratii in Chesapeake Bay populations of Gymnodinium sanguineum. Journal of Eukaryotic Microbiology, 41, pp.586-593.

Coats, D.W., Adam, E.J., Gallegos, C.L. and Hedrick, S., 1996. Parasitism of photosynthetic dinoflagellates in a shallow subestuary of Chesapeake Bay, USA. Aquatic Microbial Ecology, 11(1), pp.1-9.

Coats, D.W. and Park M.G., 2002. Parasitism of photosynthetic dinoflagellates by three strains of Amoebophrya (Dinophyta): parasite survival, infectivity, generation time, and host specificity. Journal of Phycology, 38(3), pp.520-528.

Coats, D.W., Bachvaroff, T.R. and Delwiche, C.F., 2012. Revision of the family Duboscquellidae with description of Euduboscquella crenulata n. gen., n. sp. (Dinoflagellata, Syndinea), an intracellular parasite of the ciliate Favella panamensis Kofoid \& Campbell. Journal of Eukaryotic Microbiology, 59(1), pp.1-11.

de Vargas, C., Audic, S., Henry, N., Decelle, J., Mahé, F., Logares, R., et al., 2015. Eukaryotic plankton diversity in the sunlit ocean. Science, 348(6237).

Du, X. and Peterson, W.T., 2014. Seasonal cycle of phytoplankton community composition in the coastal upwelling system off central Oregon in 2009. Estuaries and Coasts, 37(2), pp.299311.

Duboscq, O. and Collin, B. 1910. Sur la reproduction sexuée d'un protiste parasite des tintinnides. C. R. Hebd. Séances Acadé. Sci., Paris, 151, pp.340-341.

Edgcomb, V.P. (2016) Marine protist associations and environmental impacts across trophic levels in the twilight zone and below. Current Opinions in Microbiology, 31, pp.169-175.

Falkowski, P.G., Barber, R.T. and Smetacek, V., 1998. Biogeochemical controls and feedbacks on ocean primary production. Science, 281(5374), pp.200-206.

Farhat, S., Florent, I., Noel, B., Kayal, E., Da Silva, C., Bigeard, E., Alberti, A., Labadie, K., Corre, E., Aury, J.M. and Rombauts, S., 2018. Comparative time-scale gene expression analysis highlights the infection processes of two Amoebophrya strains. Frontiers in Microbiology, 9, p.2251.

Field, C.B., Behrenfeld, M.J., Randerson, J.T. and Falkowski, P., 1998. Primary production of the biosphere: integrating terrestrial and oceanic components. Science, 281(5374), pp.237-240. 
Fogg, G.E., 1983. The ecological significance of extracellular products of phytoplankton photosynthesis. Botanica Marina, 26(1), pp.3-14.

Fritz, L. and Nass, M., 1992. Development of the endoparasitic dinoflagellate Amoebophrya ceratii within host dinoflagellate species. Journal of Phycology, 28(3), pp.312-320.

Glibert, P.M., Mayorga, E. and Seitzinger, S., 2008. Prorocentrum minimum tracks anthropogenic nitrogen and phosphorus inputs on a global basis: application of spatially explicit nutrient export models. Harmful Algae, 8(1), pp.33-38.

Guidi, L., Chaffron, S., Bittner, L., Eveillard, D., Larhlimi, A., Roux, S., Darzi, Y., Audic, S., Berline, L., Brum, J.R. and Coelho, L.P., 2016. Plankton networks driving carbon export in the oligotrophic ocean. Nature, 532(7600), pp.465-470.

Gunderson, J.H., John, S.A., Chanson Boman, W. and Coats, D.W., 2002. Multiple strains of the parasitic dinoflagellate Amoebophrya exist in Chesapeake Bay. Journal of Eukaryotic Microbiology, 49(6), pp.469-474.

Higgins, M.B., Robinson, R.S., Husson, J.M., Carter, S.J. and Pearson, A., 2012. Dominant eukaryotic export production during ocean anoxic events reflects the importance of recycled $\mathrm{NH}_{4}{ }^{+}$. Proceedings of the National Academy of Sciences, 109(7), pp.2269-2274.

Hoppenrath, M., Murray, S.A., Chomérat, N., and Horiguchi, T., 2014. Marine benthic dinoflagellates - unveiling their worldwide biodiversity. - Kleine Senckenberg Reihe 54: 1-276.

Hu, S.K., Herrera, E.L., Smith, A.R., Pachiadaki, M.G., Edgcomb, V.P., Sylva, S.P., Chan, E.W., Seewald, J.S., German, C.R. and Huber, J.A., 2021. Protistan grazing impacts microbial communities and carbon cycling at deep-sea hydrothermal vents. bioRxiv.

Jacobson, D.M., 1987. The ecology and feeding biology of thecate heterotrophic dinoflagellates. Doctoral dissertation, Woods Hole Oceanographic Institution.

James, T.Y., Kauff, F., Schoch, C.L., Matheny, P.B., Hofstetter, V., Cox, C.J., Celio, G., Gueidan, C., Fraker, E., Miadlikowska, J. and Lumbsch, H.T., 2006. Reconstructing the early evolution of Fungi using a six-gene phylogeny. Nature, 443(7113), pp.818-822.

Jephcott, T.G., Alves-de-Souza, C., Gleason, F.H., Van Ogtrop, F.F., Sime-Ngando, T., Karpov, S.A. and Guillou, L., 2016. Ecological impacts of parasitic chytrids, syndiniales and perkinsids on populations of marine photosynthetic dinoflagellates. Fungal Ecology, 19, pp.47-58.

John, U., Lu, Y., Wohlrab, S., Groth, M., Janouškovec, J., Kohli, G.S., Mark, F.C., Bickmeyer, U., Farhat, S., Felder, M. and Frickenhaus, S., 2019. An aerobic eukaryotic parasite with functional mitochondria that likely lacks a mitochondrial genome. Science Advances, 5(4), p.eaav1110. 
Kagami, M., de Bruin, A., Ibelings, B.W. and Van Donk, E., 2007. Parasitic chytrids: their effects on phytoplankton communities and food-web dynamics. Hydrobiologia, 578(1), pp.113129.

Karpov, S., Mamkaeva, M.A., Aleoshin, V., Nassonova, E., Lilje, O. and Gleason, F.H., 2014. Morphology, phylogeny, and ecology of the aphelids (Aphelidea, Opisthokonta) and proposal for the new superphylum Opisthosporidia. Frontiers in Microbiology, 5, p.112.

Kayal, E., Alves-De-Souza, C., Farhat, S., Velo-Suarez, L., Monjol, J., Szymczak, J., Bigeard, E., Marie, D., Noel, B., Porcel, B.M. and Corre, E., 2020. Dinoflagellate host chloroplasts and mitochondria remain functional during Amoebophrya infection. Frontiers in Microbiology, 11, p.600823.

Keller, M., Bellows, W.K., Guillard, R.R.L., 1989. Dimethyl sulfideproduction in marine phytoplankton. In: In: Saltzman, E.S., Cooper, W.J. (Eds.), Biogenic Sulfur in the Environment. Symp. Ser. 393, American Chemical Society, Washington, DC, pp. 167-181.

Kim, S., Park, M.G., Kim, K.Y., Kim, C.H., Yih, W., Park, J.S. and Coats, D.W., 2008. Genetic diversity of parasitic dinoflagellates in the genus Amoebophrya and its relationship to parasite biology and biogeography. Journal of Eukaryotic Microbiology, 55(1), pp.1-8.

Kuhlisch, C., Schleyer, G., Shahaf, N., Vincent, F., Schatz, D. and Vardi, A., 2021. Viral infection of algal blooms leaves a unique metabolic footprint on the dissolved organic matter in the ocean. Science Advances, 7(25), p.eabf4680.

Kühn S., Medlin L., Eller G., 2004. Phylogenetic position of the parasitoid nanoflagellate Pirsonia inferred from nuclear-encoded small subunit ribosomal DNA and a description of Pseudopirsonia n. gen. and Pseudopirsonia mucosa (Drebes) comb. nov. Protist, 155(2):143-156.

Lampert, W., 1978. Release of dissolved organic carbon by grazing zooplankton. Limnology and Oceanography, 23(4), pp.831-834.

Lawrence, M.G., 1993. An empirical analysis of the strength of the phytoplanktondimethylsulfide-cloud-climate feedback cycle. Journal of Geophysical Research: Atmospheres, 98(D11), pp.20663-20673.

Li, C., Song, S., Liu, Y. and Chen, T., 2014. Occurrence of Amoebophrya spp. infection in planktonic dinoflagellates in Changjiang (Yangtze River) Estuary, China. Harmful Algae, 37, pp.117-124.

Lin, S., 2011. Genomic understanding of dinoflagellates. Research in Microbiology, 162(6), pp.551-569.

Lin, S., Cheng, S., Song, B., Zhong, X., Lin, X., Li, W., Li, L., Zhang, Y., Zhang, H., Ji, Z. and Cai, M., 2015. The Symbiodinium kawagutii genome illuminates dinoflagellate gene expression and coral symbiosis. Science, 350(6261), pp.691-694. 
Lovelock, J.E., Maggs, R.J. and Rasmussen, R.A., 1972. Atmospheric dimethyl sulphide and the natural sulphur cycle. Nature, 237(5356), pp.452-453.

Lu, Y., Wohlrab, S., Groth, M., Glöckner, G., Guillou, L. and John, U., 2016. Transcriptomic profiling of Alexandrium fundyense during physical interaction with or exposure to chemical signals from the parasite Amoebophrya. Molecular Ecology, 25(6), pp.1294-1307.

Maranda, L., 2001. Infection of Prorocentrum minimum (Dinophyceae) by the parasite Amoebophrya sp. (Dinoflagellata). Journal of Phycology, 37(2), pp.245-248.

Mazzillo, F.F., Ryan, J.P. and Silver, M.W., 2011. Parasitism as a biological control agent of dinoflagellate blooms in the California Current System. Harmful Algae, 10(6), pp.763-773.

Middelboe, M. and Lyck, P.G., 2002. Regeneration of dissolved organic matter by viral lysis in marine microbial communities. Aquatic Microbial Ecology, 27(2), pp.187-194.

Mitra, A., Flynn, K.J., Tillmann, U., Raven, J.A., Caron, D., Stoecker, D.K., Not, F., Hansen, P.J., Hallegraeff, G., Sanders, R. and Wilken, S., 2016. Defining planktonic protist functional groups on mechanisms for energy and nutrient acquisition: incorporation of diverse mixotrophic strategies. Protist, 167(2), pp.106-120.

Møller, E.F., Thor, P. and Nielsen, T.G., 2003. Production of DOC by Calanus finmarchicus, C. glacialis and $C$. hyperboreus through sloppy feeding and leakage from fecal pellets. Marine Ecology Progress Series, 262, pp.185-191.

Murray, S.A., Suggett, D.J., Doblin, M.A., Kohli, G.S., Seymour, J.R., Fabris, M. and Ralph, P.J., 2016. Unravelling the functional genetics of dinoflagellates: a review of approaches and opportunities. Perspectives in Phycology, 3(1), pp.37-52.

Neuhauser, S., Kirchmair, M., Bulman, S. and Bass, D., 2014. Cross-kingdom host shifts of phytomyxid parasites. BMC Evolutionary Biology, 14(1), pp.1-13.

Nishitani, L., Erickson, G., and Chew, K.K. (1985) Role of the parasitic dinoflagellate Amoebophrya ceratii in control of Gonyaulax catenella populations. Toxic Dinoflagellates pp.225-230.

Orsi, W., Y.C. Song, S. Hallam, and Edgcomb, V.P. 2012. Effect of oxygen minimum zone formation on communities of marine protists. The ISME Journal, 6(8), pp.1586-1601.

Pachiadaki, M.G., Taylor, C., Oikonomou, A., Yakimov, M.M., Stoeck, T. and Edgcomb, V., 2016. In situ grazing experiments apply new technology to gain insights into deep-sea microbial food webs. Deep Sea Research Part II: Topical Studies in Oceanography, 129, pp.223-231.

Pernice, M.C., Logares, R., Guillou, L. and Massana, R., 2013. General patterns of diversity in major marine microeukaryote lineages. PLoS One 8(2):e57170. 
Pomeroy, L.R., 1974. The ocean's food web, a changing paradigm. Bioscience, 24(9), pp.499504.

Raghukumar, S., 1996. Morphology, taxonomy and ecology of thraustochytrids and labyrinthulids, the marine counterparts of zoosporic fungi. In: Dayal R, editor. Advances in Zoosporic Fungi. M.D. Publications Pvt. Ltd; New Delhi: pp. 35-60.

Riebesell, U., Körtzinger, A. and Oschlies, A., 2009. Sensitivities of marine carbon fluxes to ocean change. Proceedings of the National Academy of Sciences, 106(49), pp.20602-20609.

Salomon, P.S., Janson, S. and Granéli, E., 2003. Parasitism of Dinophysis norvegica by Amoebophrya sp. in the Baltic Sea. Aquatic Microbial Ecology, 33(2), pp.163-172.

Salomon, P.S., Granéli, E., Neves, M.H. and Rodriguez, E.G., 2009. Infection by Amoebophrya spp. parasitoids of dinoflagellates in a tropical marine coastal area. Aquatic Microbial Ecology, 55(2), pp.143-153.

Scholz, B., Guillou, L., Marano, A.V., Neuhauser, S., Sullivan, B.K., Karsten, U., Küpper, F.C. and Gleason, F.H., 2016. Zoosporic parasites infecting marine diatoms-a black box that needs to be opened. Fungal Ecology, 19, pp.59-76.

Sengco, M.R., Coats, D.W., Popendorf, K.J., Erdner, D.L., Gribble, K.E. and Anderson, D.M. (2003) Biological and phylogenetic characterization of Amoebophrya sp. ex Alexandrium tamarense. In Second Symposium on Harmful Marine Algae in the US, Abstract (Vol. 57).

Selander, E., Thor, P., Toth, G. and Pavia, H., 2006. Copepods induce paralytic shellfish toxin production in marine dinoflagellates. Proceedings of the Royal Society: Biological Sciences, 273(1594), pp.1673-1680.

Sherr, E.B. and Sherr, B.F., 1994. Bacterivory and herbivory: key roles of phagotrophic protists in pelagic food webs. Microbial Ecology, 28(2), pp.223-235.

Sherr, E.B. and Sherr, B.F., 2002. Significance of predation by protists in aquatic microbial food webs. Antonie Van Leeuwenhoek, 81(1), pp.293-308.

Sherr, E.B. and Sherr, B.F., 2009. Capacity of herbivorous protists to control initiation and development of mass phytoplankton blooms. Aquatic Microbial Ecology, 57(3), pp.253-262.

Shields, J.D., Scanlon, C. and Volety, A., 2003. Aspects of the pathophysiology of blue crabs, Callinectes sapidus, infected with the parasitic dinoflagellate Hematodinium perezi. Bulletin of Marine Science, 72(2), pp.519-535.

Siano, R., Alves-de-Souza, C., Foulon, E., Bendif, E1 M. Simon, N., Guillou, L., Not, F., 2011. Distribution and diversity of Amoebophryidae parasites across oligotrophic waters of the Mediterranean Sea. Biogeosciences, 8, pp.267-278. 
Skovgaard, A., Massana, R., Balague, V. and Saiz, E., 2005. Phylogenetic position of the copepod-infesting parasite Syndinium turbo (Dinoflagellata, Syndinea). Protist, 156(4), pp.413423.

Smetacek, V., 2001. A watery arms race. Nature, 411(6839), pp.745-745.

Stat, M., Morris, E. and Gates, R.D., 2008. Functional diversity in coral-dinoflagellate symbiosis. Proceedings of the National Academy of Sciences, 105(27), pp.9256-9261.

Stefels, J., Dijkhuizen, L. and Gieskes, W.W.C., 1995. DMSP-lyase activity in a spring phytoplankton bloom off the Dutch coast, related to Phaeocystis sp. abundance. Marine Ecology Progress Series, 123, pp.235-243.

Stentiford, G.D., Neil, D.M. and Coombs, G.H., 2000. Alterations in the biochemistry and ultrastructure of the deep abdominal flexor muscle of the Norway lobster Nephrops norvegicus during infection by a parasitic dinoflagellate of the genus Hematodinium. Diseases of Aquatic Organisms, 42(2), pp.133-141.

Stentiford, G.D., Chang, E.S., Chang, S.A. and Neil, D.M., 2001. Carbohydrate dynamics and the crustacean hyperglycemic hormone $(\mathrm{CHH})$ : effects of parasitic infection in Norway lobsters (Nephrops norvegicus). General and Comparative Endocrinology, 121(1), pp.13-22.

Stentiford, G.D. and Shields, J.D. (2005) A review of the parasitic dinoflagellates Hematodinium species and Hematodinium-like infections in marine crustaceans. Diseases of Aquatic Organisms, 66(1), pp.47-70.

Stoecker, D.K., 1999. Mixotrophy among dinoflagellates. Journal of Eukaryotic Microbiology, 46(4), pp.397-401.

Strassert, J.F., Karnkowska, A., Hehenberger, E., del Campo, J., Kolisko, M., Okamoto, N., Burki, F., Janouškovec, J., Poirier, C., Leonard, G. and Hallam, S.J., 2017. Single cell genomics of uncultured marine alveolates shows paraphyly of basal dinoflagellates. The ISME Journal, $12(1)$, p.304.

Sunagawa, S., Coelho, L.P., Chaffron, S., Kultima, J.R., Labadie, K., Salazar, G., Djahanschiri, B., Zeller, G., Mende, D.R., Alberti, A. and Cornejo-Castillo, F.M., 2015. Structure and function of the global ocean microbiome. Science, 348(6237), p.1261359.

Taylor, A.C., Field, R.H. and Parslow-Williams, P.J., 1996. The effects of Hematodinium sp.infection on aspects of the respiratory physiology of the Norway lobster, Nephrops norvegicus. Journal of Experimental Marine Biology and Ecology, 207(1-2), pp.217-228.

Taylor, G.T., Karl, D.M., and Pace, M.L., 1986. Impact of bacteria and zooflagellates on the composition of sinking particles: An in-situ experiment. Marine Ecology Progress Series, 29, pp.141-155. 
Tamigneaux, E., Mingelbier, M., Klein, B. and Legendre, L., 1997. Grazing by protists and seasonal changes in the size structure of protozooplankton and phytoplankton in a temperate nearshore environment (western Gulf of St. Lawrence, Canada). Marine Ecology Progress Series, 146, pp.231-247.

Tillmann, U., Alpermann, T., John, U. and Cembella, A., 2008. Allelochemical interactions and short-term effects of the dinoflagellate Alexandrium on selected photoautotrophic and heterotrophic protists. Harmful Algae, 7(1), pp.52-64.

Torres-Beltrán, M., Sehein, T., Pachiadaki, M., Hallam, S.J. and Edgcomb, V., 2018. Protistan parasites along oxygen gradients in a seasonally anoxic fjord: a network approach to assessing potential host-parasite interactions. Deep Sea Research Part II: Topical Studies in Oceanography, 156, pp.97-110.

Turner, S.M., Malin, G., Liss, P.S., Harbour, D.S. and Holligan, P.M., 1988. The seasonal variation of dimethyl sulfide and dimethylsulfoniopropionate concentrations in nearshore waters. Limnology and Oceanography, 33(3), pp. 364-375.

Van Boekel, J.S.W. and Stefels, W.H.M., 1993. Production of DMS from dissolved DMSP in axenic cultures of the marine phytoplankton species Phaeocystis sp. Marine Ecology Progress Series, 97, pp.11-18.

Van Donk, E., 1989. The role of fungal parasites in phytoplankton succession. In Plankton ecology (pp. 171-194). Springer, Berlin, Heidelberg.

van Duyl, F.C., Gieskes, W.W., Kop, A.J. and Lewis, W.E., 1998. Biological control of shortterm variations in the concentration of DMSP and DMS during a Phaeocystis spring bloom. Journal of Sea Research, 40(3-4), pp.221-231.

Velo-Suárez L., Brosnahan, M. L., Anderson, D. M., McGillicuddy, D. J. Jr., 2013. A quantitative assessment of the role of the parasite Amoebophrya in the termination of Alexandrium fundyense blooms within a small coastal embayment. PLoS One, 8(12), e81150.

Verity, P.G., Stoecker, D.K., Sieracki, M.E. and Nelson, J.R., 1993. Grazing, growth and mortality of microzooplankton during the 1989 North Atlantic spring bloom at $47^{\circ} \mathrm{N}, 18^{\circ} \mathrm{W}$. Deep Sea Research Part I: Oceanographic Research Papers, 40(9), pp.1793-1814.

Wisecaver, J.H. and Hackett, J.D., 2011. Dinoflagellate genome evolution. Annual Review of Microbiology, 65, pp.369-387.

Worden, A.Z., Follows, M.J., Giovannoni, S.J., Wilken, S., Zimmerman, A.E. and Keeling, P.J., 2015. Rethinking the marine carbon cycle: factoring in the multifarious lifestyles of microbes. Science, 347(6223), pp.735-735. 
Zehr, J.P. and Voytek, M. eds., 2012. Molecular ecology of aquatic communities (Vol. 138). Springer Science \& Business Media.

Zhao, Z., Gonsior, M., Schmitt-Kopplin, P., Zhan, Y., Zhang, R., Jiao, N. and Chen, F., 2019. Microbial transformation of virus-induced dissolved organic matter from picocyanobacteria: coupling of bacterial diversity and DOM chemodiversity. The ISME Journal, 13(10), pp.25512565 . 


\section{CHAPTER ONE}

Protistan parasites along oxygen gradients in a seasonally anoxic fjord: a network approach to assessing potential host-parasite interactions

Mónica Torres-Beltrán ${ }^{1 *}$, Taylor Sehein ${ }^{2 *}$, Maria Pachiadaki ${ }^{2,3}$, Steven J. Hallam ${ }^{1,4,5,6}$, Virginia Edgcomb $^{2 \wedge}$

${ }^{1}$ Department of Microbiology \& Immunology, University of British Columbia, Vancouver, British Columbia, Canada

${ }^{2}$ Geology and Geophysics Department, Woods Hole Oceanographic Institute, Woods Hole, MA, USA.

${ }^{3}$ Bigelow Laboratory for Ocean Sciences, East Boothbay, ME, USA

${ }^{4}$ Graduate Program in Bioinformatics, University of British Columbia, Vancouver, British Columbia, Canada

${ }^{5}$ Peter Wall Institute for Advanced Studies, University of British Columbia, Canada

${ }^{6}$ ECOSCOPE Training Program, University of British Columbia, Vancouver, British Columbia, Canada

*Authors contributed equally to this work

^ Correspondence: Virginia Edgcomb, vedgcomb@whoi.edu

Key words:

Syndiniales, parasitic protists, network analyses, OMZ, stratified water column

Originally Published in Deep Sea Research Part II: Topical Studies in Oceanography https://doi.org/10.1016/j.dsr2.2017.12.026

\section{STATEMENT OF CONTRIBUTION}

Samples were collected and processed by Mónica Torres-Beltrán with Steven J. Hallam and other members of the Hallam Lab. Sequence data was processed and co-occurrence networks were constructed by MTB. TS, MTB, VE, MP and SJH analyzed and interpreted the cooccurrence network data. TS selected taxa of interest associated with syndinian parasites and wrote portions of the manuscript describing these associations. TS, MTB, SJH, and VE wrote and edited the paper. 


\begin{abstract}
The seasonally stratified water column of Saanich Inlet, Vancouver, B.C., Canada provides a natural laboratory for examining how ocean deoxygenation shapes microbial community population dynamics and interactions. Microbial eukaryotes within these communities play significant roles as phototrophs, mixotrophs, symbionts, predators, parasites, and saprotrophs, with population abundances and interactions changing seasonally, and along water column redox gradients. Here, we use time series observations of ribosomal RNA and DNA diversity in combination with co-occurrence correlation and indicator species analyses to observe microbial eukaryote community dynamics over a 12-month period, uncovering significant correlations between parasitic dinoflagellates within Syndiniales and other eukaryotic taxa during months of peak water column stratification. Syndiniales operational taxonomic units (OTUs) were the most abundant indicator OTUs during stratification. Syndiniales OTUs exhibited significant correlations with a broad range of taxa, including members of Apicomplexa, Ciliophora, Dinoflagellata, Chlorophyta, Metazoa and a variety of Stramenopiles including MAST groups. Correlation analyses suggest Syndiniales may influence phytoplankton bloom dynamics in Saanich Inlet as well as abundances of protist and metazoan grazers that are indicator species for peak stratification periods. These observations shed light on the potential role of parasitic protists on organic matter release and bloom termination events during stratification and renewal periods in Saanich Inlet with potential implications for coastal marine food webs and fisheries productivity.
\end{abstract}

\title{
INTRODUCTION
}

Marine oxygen minimum zones (OMZs) are regions in the ocean where oxygen concentrations range between $\sim 20 \mu \mathrm{M}$ and undetectable levels (Ulloa and Pantoja, 2009). OMZs cover about $8 \%$ of ocean surface area (Paulmier and Ruiz-Pino, 2009) and $\sim 1 \%$ of current ocean volume (Ulloa and Pantoja, 2009). The vertical and horizontal extent of these regions has increased over the last five decades (Schmidtko et al., 2017; Stramma et al., 2008). OMZs are typically located near eastern boundary upwelling systems, and some of the most prominent include the northern Indian Ocean and Arabian Sea, and the eastern north and south Pacific. Coastal OMZs also occur in locations where water circulation is reduced and/or oxygen-depleted conditions are exacerbated by anthropogenic additions of nutrients to coastal waters. As oxygen levels decline, energy in OMZs is increasingly diverted into microbial community metabolism with resulting 
feedback on metazoan organisms through habitat compression. This has a disproportionate impact on large multicellular eukaryotes that cannot permanently occupy anoxic OMZ cores (Parris et al., 2014). Despite this tendency, some vertically-migrating crustacea, zooplankton, chaetognaths and fish are known to seek temporary refuge from predation in OMZ waters (Escribano et al., 2009; Wishner et al., 1998; Wishner et al., 2013). Moreover, a diverse community of microeukaryotes and zooplankton can operate in OMZ waters where they help shape biogeochemistry and ecology. Protists in particular, participate in wide-ranging interactions that couple metabolic processes and behaviour to nutrient and energy flow patterns in the environment (Arístegui et al., 2009).

Throughout marine water columns and sediments, protists shape pools of bioavailable carbon and other nutrients through production, grazing and symbiotic associations. Phagotrophic protists and viruses are considered as the main sources of mortality for marine organisms (Arístegui et al., 2009; Suttle, 2005). Organic carbon in the dissolved fraction is transferred to higher trophic levels (to Metazoa) in a heterotrophic food chain involving bacteria, small and large nanoflagellates $(2-20 \mu \mathrm{m})$, and larger flagellates and ciliates, termed the "microbial loop" (Azam et al., 1983; Taylor GT, 1986). The relative contributions of protist grazing and viral lysis as top-down controls on marine bacterioplankton are still debated (e.g. (Chow et al., 2014; Cuevas and Morales, 2006; Pedrós-Alió et al., 2000), and undoubtedly vary depending on sitespecific physico-chemical conditions and the physiological state of individual populations. Diverse communities of phagotrophic and parasitic protists have been described under low oxygen conditions, many of which exhibit putative symbiotic relationships with prokaryotes, and protist grazing has been shown to shape specific prey populations along the redoxcline (Lin et al., 2007; Edgcomb et al., 2011; Orsi et al., 2011, 2012; Wright et al., 2012; Parris et al. 2014; Jing et al. 2015).

Parasitic protists are taxonomically diverse and a major source of mortality for other microbial eukaryotes as well as Metazoa (Chambouvet et al., 2008), likely making them important shapers of food web structure (Jephcott et al., 2016). The Alveolata comprise one of the largest eukaryotic lineages, which includes four protist groups, the Ciliophora, Dinoflagellata, Protalveolata, and Apicomplexa, the last two of which are comprised primarily of parasites (Edgcomb, 2016). Exclusive marine parasitoid protists consistently recovered in highthroughput molecular studies were initially assigned to novel marine alveolate (MALV) Groups I 
and II within the Syndiniales (Edgcomb et al., 2011; Lopez-Garcia et al., 2001; Massana et al., 2004b; Moon-van der Staay et al., 2001; Not et al., 2007; Romari and Vaulot, 2004). Group I consists of numerous undescribed species while Group II contains sequences affiliated with the genus Amoebophrya, including species influencing 'red tide' blooms (Chambouvet et al., 2008). Over time the original two groups have expanded to include five (Guillou et al., 2008) or eight (Richards and Bass, 2005) deeply diverging and abundant clades. For example, in euphotic zone samples from the Tara Oceans expedition $36 \%$ of protistan operational taxonomic units (OTUs) were attributed to parasites, almost half of which were affiliated with a single MALV Group I clade (de Vargas et al., 2015). Molecular signatures of Syndiniales are almost universally retrieved from picoplankton $(<3 \mathrm{um})$ samples, although some clades within these groups appear to be restricted to specific habitats (Guillou et al., 2008). Variations in sequence abundances in ribosomal RNA- vs. DNA-based libraries may indicate variability in the impact of parasitism in different habitats; however, read abundances do not necessarily equate to cell concentrations due to PCR amplification biases and variations in taxon-specific rDNA copy numbers (Not et al., 2009).

Infection of host cells by members of Syndiniales typically leads to host mortality. Known hosts include other Syndiniales, photosynthetic dinoflagellates, ciliates, rhizarians and metazoans (Bråte et al., 2012; Guillou et al., 2008; Massana et al., 2014). The most studied syndinian parasites belong to the species complex, Amoebophrya. Amoebophrya infect new hosts by forming motile spores or weakly motile spores (dinospores) that penetrate the host cell membrane and travel to the host nucleus or cytoplasm to replicate (Chambouvet et al., 2008). Over the course of several days, the trophont grows and ultimately ruptures the host cell membrane, releasing the ephemeral, multinucleate, (often) multiflagellate parasite vermiform into the water column, which subsequently fragments into individual dinospores (Chambouvet et al., 2008). In the case of some syndinian parasites (e.g., infection of tintinnid ciliates by Euduboscquella species), infection is thought to be a passive process, whereby the host ingests but does not digest the spores (Dolan, 2013). Other syndinian trophonts, such as those formed by Euduboscquella species emerge through a more elaborate process involving creation of a food vacuole encompassing the remains of the host cell, digestion of host remains, followed by serial nuclear and cytoplasmic divisions to form spores (Coats et al., 2012; Dolan, 2013). Increased syndinian parasite abundance during the decline of harmful algal blooms (i.e. Alexandruim 
catanella) suggest potential top-down control of phytoplankton species during bloom events (Bai et al., 2007; Choi et al., 2017; Velo-Suárez et al., 2013a). Studies of parasitism of other taxa suggest syndinian parasites infect their hosts throughout the year, but studies of ciliates indicate that infections of ciliate hosts are most common in summer months when phytoplankton biomass is apparently sufficient to support elevated grazer densities (Cachon, 1964; Coats, 1989; Coats et al., 1994). Thus, Syndiniales may have the potential to contribute significantly to releases of organic carbon through cell lysis during bloom termination events and may influence protist community diversity by exerting top-down control on select species within a population.

Fluorescence in situ hybridization (FISH) analyses has shown that Syndiniales alternate between two life stages: the free-swimming, infective dinospore that can survive without a host for up to three days, and the multinuclear trophont phase within a host (Velo-Suárez et al., 2013b). Most infections lead to lysis of the host cell; however, one study of the Syndiniales Group II taxon Amoebophrya, revealed dinospores could lie dormant within the resting stage of the dinoflagellate, Scrippsiella trochoidea and re-emerging simultaneously when the host entered the vegetative state, suggesting potentially complex life histories that can impact protist populations at multiple life stages (Chambouvet et al., 2011). In molecular surveys of protistan diversity, Syndiniales are grouped taxonomically with the novel marine alveolate (MALV) lineages I and II and can form the majority of sequences from diverse marine habitats including coastal waters, open oceans, and stratified systems (Guillou et al., 2008; Massana et al., 2004a; Not et al., 2007). These parasites are thought to exhibit top-down pressures on host populations within the eukaryotic community, such as, radiolarians, dinoflagellates, ciliates, and metazoa; however, there is a paucity of data on the range of host species for different Syndiniales groups or on net ecosystem effects of parasitism by these taxa.

Saanich Inlet is a fjord on the coast of Vancouver Island British Columbia that undergoes seasonal stratification resulting in progressive deoxygenation followed by deep-water renewal events that inject oxygen and nutrients into deep basin waters. The Inlets' seasonally stratified water column serves as a well-characterized model ecosystem for examining how deoxygenation shapes microbial community population dynamics and interactions along defined redox gradients in the ocean. Stratification and deep-water renewal cycles are known to shape microbial community composition and activity, and to lead to seasonal blooms of not only various phytoplankton (some of which are toxic species) but also metazoan species that serve as prey for 
commercially important fisheries in the area. A previous study indicated that protist populations occupy different niches along the redoxcline over a 12-month cycle including abundant Dinoflagellata OTUs (Orsi et al. 2012). In oxic waters at 10m depth, Syndiniales OTUs represented $\sim 90 \%$ of all Dinoflagellata-affiliated sequences in samples from all seasons (Orsi et al. 2012). At 200m depth an increase in Dinoflagellata OTUs (80\% affiliated with Syndiniales) was observed during deep water renewal periods (Orsi et al. 2012). While this may reflect active infection of a deep-water bloom of non-phototrophic hosts, this may alternatively reflect dinospores released from sinking infected and lysed phototrophic hosts originating from the upper water column as a response to seasonal blooms.

Here we explore eukaryotic small subunit ribosomal RNA- and DNA-based pyrotag datasets generated from monthly filtered samples collected at depths throughout the Saanich Inlet water column over a 12-month period to elucidate changes in microbial eukaryote populations and eukaryote-eukaryote interactions that have the potential to influence OMZ biogeochemistry and ecology. We focus on population dynamics of Syndiniales OTUs to better constrain their impact on organic matter release and bloom termination during seasonal stratification and renewal.

\section{METHODS}

\section{Environmental sampling}

Environmental monitoring and sample collection were carried out once per month aboard the MSV John Strickland at station SI03 (480 35.500 N, 123030.300 W) in Saanich Inlet, B.C. From May 2008 to April 2009 and July 2014 a total of 175 water samples were collected at 16 high-resolution depths $(10,20,40,60,75,80,90,97,100,110,120,135,150,165,185$ and 200

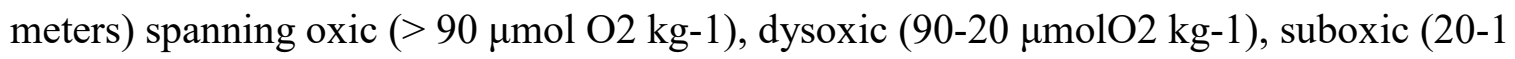
$\mu \mathrm{molO} 2 \mathrm{~kg}-1)$ anoxic $(<1 \mu \mathrm{mol} \mathrm{O} 2 \mathrm{~kg}-1)$ and sulfidic water column conditions (Wright et al., 2012). Samples were processed and analyzed as previously reported for the Saanich Inlet timeseries (Hawley et al., 2017; Torres-Beltrán et al., 2017; Zaikova et al., 2010). Briefly, water samples were collected from Niskin or Go-Flow bottles for DNA and RNA (Hawley et al., 2017). Conductivity, temperature, and depth were measured using a Seabird SBE19 CTD-device (Sea-Bird Electronics Inc., Bellevue USA), with a PAR and O2 sensor attached. An oxygen probe attached to the CTD was also used to measure dissolved $\mathrm{O} 2$ throughout the water column as previously described (Torres-Beltrán et al., 2017). 
Nucleic acid sampling and extraction

Biomass to generate SSU rDNA pyrotag datasets for microbial community composition profiling (2L) was filtered directly onto a $0.22 \square \mathrm{m}$ Sterivex polycarbonate cartridge filter from highresolution depths collected from May 2008 to April 2009, and July 2014. Biomass to generate SSU rRNA pyrotag datasets for potentially active microbial community composition profiling analysis (2L) was collected on July 2014 at high-resolution depths and filtered directly onto a $0.22 \square \mathrm{m}$ Sterivex polycarbonate cartridge filter within 20 minutes of shipboard sample collection.

DNA was extracted from the Sterivex filters as previously described (Hawley et al., 2017; Zaikova et al., 2010). Briefly, after defrosting the filter on ice, $100 \mu 1$ lysozyme $(0.125 \mathrm{mg}$

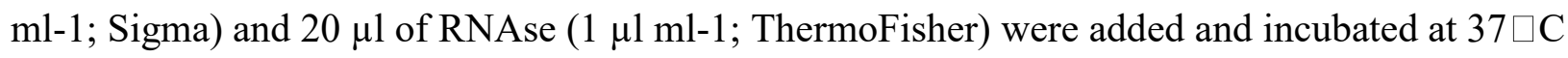
for $1 \mathrm{~h}$ with rotation followed by addition of $50 \mu 1$ Proteinase K (Sigma) and $100 \mu 1$ 20\% SDS and incubated at $55 \square \mathrm{C}$ for $2 \mathrm{~h}$ with rotation. Lysate was removed by pushing through with a syringe into $15 \mathrm{~mL}$ falcon tube (Corning) and with an additional rinse of $1 \mathrm{~mL}$ of lysis buffer. Filtrate was subject to chloroform extraction (Sigma) and the aqueous layer was collected and loaded onto a 10K $15 \mathrm{ml}$ Amicon filter cartridge (Millipore), washed three times with TE buffer ( $\mathrm{pH}$ 8.0) and concentrated to a final volume of between 150-400 $\mu 1$. Total DNA concentration was determined by PicoGreen assay (Life Technologies) and genomic DNA quality determined by visualization on $0.8 \%$ agarose gel (overnight at $16 \mathrm{~V}$ ).

RNA was extracted from the Sterivex filters using a modified protocol (Hawley et al., 2017) of the mirVana Isolation kit (Ambion) (Shi et al., 2009; Stewart et al., 2010). Briefly, after thawing the filter cartridge on ice RNAlater was removed by pushing through with a $3 \mathrm{ml}$ syringe followed by rinsing with an additional $1.8 \mathrm{~mL}$ of Ringer's solution and incubated at room temperature for $20 \mathrm{~min}$ with rotation. Ringer's solution was evacuated with a $3 \mathrm{ml}$ syringe followed by addition of $100 \mu \mathrm{l}$ of $0.125 \mathrm{mg} \mathrm{ml}-1$ lysozyme and incubated at $37^{\circ} \mathrm{C}$ for $30 \mathrm{~min}$ with rotation. Lysate was removed from the filter cartridge and subjected to organic extraction following the mirVana kit protocol. DNA removal and clean up and purification of total RNA were conducted following the TURBO DNA-free kit (ThermoFisher) and the RNeasy MinElute Cleanup kit (Qiagen) protocols respectively. Total RNA concentration was determined by RiboGreen analysis (Life Technologies) prior to first strand synthesis of cDNA using the 
SuperScript III First-Strand Synthesis System for RT-qPCR (Invitrogen) according to manufacturer instructions.

Small subunit ribosomal $R N A$ and $R N A$ gene sequencing and analysis

All pyrotag libraries were generated by PCR amplification using multi-domain primers targeting the V6-V8 region of the SSU rRNA gene (Allers et al., 2013): 926F (5'-cct atc ccc tgt gtg cct tgg cag tct cag AAA CTY AAA KGA ATT GRC GG-3') and 1392R (5'-cca tct cat cce tgc gtg tct ccg act cag- $<$ XXXXX $>$-ACG GGC GGT GTG TRC-3'). Primer sequences were modified by the addition of $454 \mathrm{~A}$ or $\mathrm{B}$ adapter sequences (lower case). In addition, the reverse primer included a 5 bp barcode designated $<\mathrm{XXXXX}>$ for multiplexing of samples during sequencing. Twenty-five microliter PCR reactions were performed in triplicate and pooled to minimize PCR bias. Each reaction contained between 1 and $10 \mathrm{ng}$ of target DNA, $0.5 \mu \mathrm{l}$ Taq DNA polymerase (Bioshop inc. Canada), $2.5 \mu \mathrm{L}$ Bioshop $10 \mathrm{x}$ buffer, $1.5 \mathrm{uL} 25 \mathrm{mM}$ Bioshop MgCl2, $2.5 \mu \mathrm{L} 10$ $\mathrm{mM}$ dNTPs (Agilent Technologies) and $0.5 \mu \mathrm{L} 10 \mathrm{mM}$ of each primer. The thermal cycler protocol started with an initial denaturation at $95^{\circ} \mathrm{C}$ for 3 minutes, followed by 25 cycles of $30 \mathrm{~s}$ at $95^{\circ} \mathrm{C}, 45 \mathrm{~s}$ at $55^{\circ} \mathrm{C}, 90 \mathrm{~s}$ at $72^{\circ} \mathrm{C}$ and $45 \mathrm{~s}$ at $55^{\circ} \mathrm{C}$, and a final extension at $72^{\circ} \mathrm{C}$ for $10 \mathrm{~min}$. PCR products were purified using the QiaQuick PCR purification kit (Qiagen), eluted ( $25 \mu \mathrm{L})$, and quantified using PicoGreen assay (Life Technologies). SSU rRNA and SSU rRNA gene (rDNA) amplicons were pooled at $100 \mathrm{ng}$ for each sample. PCR amplicons were sequenced on Roche 454 GS FLX Titanium at the McGill University and Génome Québec Innovation Center.

Pyrotag sequences from May 2008 to April 2009 were processed using the Quantitative Insights Into Microbial Ecology (QIIME) software package (Caporaso et al., 2010). To minimize the removal of false positive reads all 2,501,489 pyrotag sequences generated from the 159 samples during this time interval were clustered together. Reads with a length shorter than 200 bases, ambiguous bases, and homopolymer sequences were removed prior to chimera detection. Chimeras were detected and removed using chimera slayer provided in the QIIME software package. Sequences were then clustered into operational taxonomic units (OTUs) at 97\% identity using uclust with average linkage algorithm. Prior to taxonomic assignment singleton OTUs (OTUs represented by one read) were omitted, leaving 251,337 protistan OTUs. Representative sequences from each non-singleton OTU were queried against the SILVA database (Pruesse et al., 2007) using BLAST (Altschul et al., 1990). Pyrotag sequences from July 2014 were recruited 
to the OTUs sequences from the May 2008 to April 2009 dataset using BLAST (Altschul et al., 1990) with an identity threshold higher than 98\%. Only protistan OTUs showing the highest match ( $>98 \%$ identity) across the reference dataset were selected to support their potential activity under defined water column conditions.

\section{Statistical analyses}

Unless otherwise indicated all statistical analyses were performed in the R software environment (RCoreTeam, 2013). Pyrotag datasets were Hellinger transformed (Legendre and Gallagher, 2001) using the vegan package to the square root of observed total number of reads per sample. Hierarchical cluster analysis (HCA) was conducted to identify groups associated with discrete water column conditions using the pvclust (Suzuki and Shimodaira, 2015) package with Manhattan Distance measures, and statistical significance to the resulting clusters was computed as bootstrap score distributions with 1,000 iterations.

Multi-level indicator species analysis (ISA) using the indicspecies package (De Caceres and Legendre, 2009) was performed to identify OTUs specifically associated with different water column conditions defined by HCA that may be known by their parasite-host interaction. The ISA/multi-level pattern analysis calculates $\mathrm{p}$ values with Monte Carlo simulations and returns indicator values (IV) and p-values $\leq 0.05$. The IVs range between 0 and 1 , where indicator OTUs considered in the present study for further community analysis show an IV $\geq 0.6$ and $\mathrm{p}$-value $\leq 0.001$

To generate a robust matrix of significant protistan co-occurrences between prevalent OTUs at water column conditions that may represent parasite-host interactions, correlation coefficients from May 2008 to April 2009 pyrotag samples were calculated using the Bray-Curtis and Spearman's rank correlations in the CoNet software (Faust et al., 2012) with OTU abundance as count data as previously used for pyrotag data (Torres-Beltrán et al., 2016). Prior to analysis in CoNet, the OTU matrix derived from pyrotag taxonomic analysis was transformed into presence-absence data to remove OTUs with less than 1/3 zero counts, leaving 556 OTUs for all samples that were selected in the OTU abundance matrix for correlation analysis. To construct ensemble networks, thresholds for Bray-Curtis and Spearman's rank correlations measures were set to 0.6 correlation value as a pre-filter for OTU pairs, followed by computing edge scores only between highly correlated OTU pairs. To assign statistical significance to the 
resulting scores, edge and correlation measure-specific permutation and bootstrap score distributions with 1,000 iterations each were computed. P-values were tail-adjusted so that low p-values correspond to co-presence and high p-values to exclusion, $p$-values on each final edge were corrected to q-values (cut-off of 0.05). Finally, only edges with at least two supporting pieces of evidence i.e. high correlation value on both correlation measures and q-value below threshold were retained. The final edges and nodes matrices were exported using Cytoscape 2.8.3 (Shannon et al., 2003). Nodes corresponded to individual OTUs and edges were defined by computed correlations between corresponding OTU pairs. Edges were selected and exported based on specific paired OTUs such as those affiliated with parasite-host taxonomic groups.

To gain insight into potential parasite-host activity, SSU rRNA:rDNA ratios were calculated as previously described for pyrosequencing data (Stewart et al., 2012). We selected OTUs from the July 2014 dataset based on their taxonomic affiliation (BLAST-based $>98 \%$ similarity) with taxa of interest. In addition, only OTUs affiliated to indicators showing significant correlations were selected.

\section{Data deposition}

The SSU rDNA and rRNA pyrotag sequences reported in this study have been deposited in the NCBI under the accession numbers: SAMN03387532 - SAMN03387915 and SAMN05392441 SAMN05392453.

\section{RESULTS}

\section{Water column conditions}

Water column properties were monitored through the progression of stratification and deepwater renewal over a one-year period (May 2008 to April 2009). Throughout the year the water column surface temperature ranged between 7 to $16^{\circ} \mathrm{C}$ during winter and summer months respectively, reaching an average of $9^{\circ} \mathrm{C}$ at bottom waters. Salinity between surface and bottom waters ranged between 28 to 31 ppt throughout the year, showing the lowest values at the surface during spring months. In addition, as previously described for OMZs (Wright et al., 2012), in this study we define water column conditions on the basis of $\mathrm{O} 2$ concentration ranges: oxic $(>90 \mu \mathrm{M}$

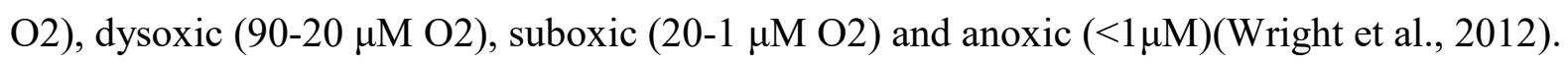
Between May and August 2008, as water column stratification peaked, suboxic conditions intensified corresponding with the development of deep-water anoxia. The concentration of 
NO3- between the surface and $100 \mathrm{~m}$ ranged between 5 to $20 \mu \mathrm{M}$, decreasing rapidly between 100 and $135 \mathrm{~m}$ before reaching a minimum of $\leq 1 \mu \mathrm{M}$ in anoxic bottom waters (Fig 1). The beginning of 2008 deep-water renewal occurred toward the end of September and continued through November. Initially, dissolved O2 was observed throughout the water column, although upwards shoaling of $\mathrm{O} 2$ and $\mathrm{NO} 3$ - depleted bottom waters produced an intermediate suboxic layer between 100 and 135m. By October, dissolved O2 concentrations between 150 and 200m ranged between 14 and $28 \mu \mathrm{M}$ consistent with complete oxygenation of the water column. Over the same time interval, NO3- concentrations between surface and 100m ranged between 4 to 29 $\mu \mathrm{M}$, decreasing rapidly below $100 \mathrm{~m}$ before reaching a second maximum ranging between 21 to $24 \mu \mathrm{M}$ at 200m (Fig 1). In November, O2 and NO3-concentrations continued to increase above 100 and below $135 \mathrm{~m}$ with intervening depth intervals experiencing increased oxygen decline. In December water column O2 deficiency intensified below $100 \mathrm{~m}$ as the water column become increasingly stratified through April 2009.

\section{Eukaryotic community structure}

To explore eukaryotic community structure in the Saanich Inlet water column we analyzed SSU rRNA gene pyrotag sequences from 159 samples collected at 16 depths ranging between 10 and 200m over the time interval between May 2008 and April 2009. Overall, the opisthokont community was dominated by OTUs affiliated with metazoan sequences within the phylum Arthropoda (34\% total community) (Fig 2A). Protistan community composition was dominated by OTUs affiliated with taxonomic groups previously identified in the Saanich Inlet water column, using different identification approaches (Orsi et al., 2012) including the Alveolata (41\%), Stramenopiles (10\%), Hacrobia (3\%) and Rhizaria (2\%) (Fig 2A). Nineteen percent of Alveolata OTUs were affiliated with Dinoflagellata sequences within Syndiniales groups (I, II, III and V) (Fig 2B). Groups I (27\% Syndiniales OTUs) and II (66\% Syndiniales OTUs) dominated throughout the year showing peaks of abundance during the stratification period (May- August) in the inlet (Fig 3B) reaching up to $19 \%$ and $43 \%$ of relative abundance, respectively, from the total protist sequences.

To describe protistan community partitioning along water column $\mathrm{O} 2$ gradients we conducted hierarchical clustering analysis using rDNA pyrotag profiles from each sample. Results revealed four major groups or clusters ( $\mathrm{AU} \geq 70,1,000$ iterations) associated with oxic 
(group I), dysoxic-suboxic (group II and III), and anoxic (group IV) water column conditions that were previously observed to occur with seasonal stratification and deep water renewal events (Orsi et al., 2012) (Fig 3A). To further resolve OTUs occurring under specific water column $\mathrm{O} 2$ conditions and to reveal protistan co-occurrence patterns between taxa i.e. Syndiniales and potential hosts, multi-level indicator species analysis (ISA) was conducted based on HCA groups (Supplementary Information Table S1-4). Indicator OTUs affiliated with Arthropoda, Cnidaria, Dinoflagellata and Stramenopiles were characteristic of the oxic water column compartment while indicator OTUs affiliated with Choanoflagellatea, Colpodea, Cryptophyceae, Dinoflagellata, Picozoa, Prymnesiophyceae, Stramenopiles and Telonemia were characteristic of the dysoxic-suboxic water column conditions (Fig 3B). Syndiniales OTUs were the most abundant indicator OTUs found in these water column conditions during peak stratification in summer (56\% of total indicator OTUs) (Fig 3C). In addition, OTUs affiliated with potential hosts such as the ciliate subclass Choreotrichia and the metazoan class Maxillopoda, were also found as indicators for the dysoxic-suboxic water column conditions along with the Syndiniales OTUs. In comparison, indicator OTUs affiliated with Dinoflagellata, Stramenopiles and Arthropoda were identified for the anoxic water column compartment during the stratification period.

\section{Exploring protistan co-occurrence patterns}

To explore community co-occurrence patterns focusing primarily on potential parasitic interactions between Syndiniales and protists throughout water column O2 gradients, we conducted a co-occurrence analysis based on Bray-Curtis and Spearman correlations among OTUs. Resulting significant (Correlation Value $>0.6, \mathrm{p}<0.001$ ) pairs were used to determine potential interactions i.e. parasitism dynamics over water column stratification period.

Correlation analysis resulted in a total of 6,273 significant pairs corresponding to co-occurrences between prevalent OTUs throughout water column conditions over time. Significant pairs were observed among different taxonomic groups suggesting a broad range of potential interactions resulting from 325 unique source OTUs affiliated with Alveolata (3,765), Apusozoa (3), Archeaplastida (139), Excavata (250), Hacrobia (483), Opisthokonta (418), Rhizaria (224), and Stramenopiles (986). 
Syndiniales correlations corresponded to $63 \%$ of the total pairs observed, showing cooccurrence with OTUs affiliated with Alveolata (including Apicomplexa, Ciliophora, Dinoflagellata), Chlorophyta, Metazoa and a variety of Stramenopiles including MAST groups (Fig 4; Supplementary Information S1 and Table S5). The relative proportion of taxa correlating with Syndiniales OTUs was similar between groups; however, the number of unique OTUs associated with the different Syndiniales groups varied significantly. For instance, group II was the most taxonomically diverse assemblage, exhibited the most correlations with other protistan OTUs, while Group V exhibited the fewest (Fig 5A). The occurrence of OTUs affiliated with amoebae, green algae, haptophytes, and Metazoa was exclusively statistically correlated with Syndiniales groups I and II (Fig 5B). Interestingly, significant positive correlations between Syndiniales and OTUs affiliated with taxa described as potential hosts i.e. Choreotrichia, Phaeocystis sp. and Maxillopoda, were observed in different oxygen regimes. For instance, while Syndiniales (groups I-V) and Choreotrichia OTUs co-occurred throughout the water column, Syndiniales groups II, III and V, and Chroreotrichia OTUs co-occurred mostly in dysoxicsuboxic (90-150m) and anoxic (below 150m) water column conditions as water column stratification intensified during summer months (Fig 6). Syndiniales-Choreotrichia co-occurrence patterns were supported by ISA results showing these OTUs to be characteristic of dysoxicsuboxic water column conditions during the same time intervals (Supplementary Table S1 and S3). In addition, Syndiniales groups I and II, and Phaeocystis sp. OTUs co-occurred in oxic waters in May and become progressively associated with dysoxic-suboxic waters as the water column became increasingly stratified in July and August (Fig 7). Syndiniales groups I and II, and Maxillopoda OTUs exhibited a similar temporal co-occurrence pattern (Fig 8) that were supported by ISA results indicating that these OTUs were characteristic of oxic and dysoxic water column conditions during the same time intervals (Supplementary Table S1 and S3). Moreover, we observed that OTUs affiliated with Syndiniales and the three target taxa were evenly distributed throughout the water column in September and October possibly reflecting vertical transport and mixing during deep water renewal.

Insight into potential Syndiniales parasitic interactions Given the observed co-occurrence patterns between rDNA of Syndiniales and Choreotrichia, Phaeocystis sp. and Maxillopoda during the 2008 stratification period, we wanted to evaluate the 
potential activity of these groups under low oxygen conditions. We made this connection by comparing pyrotag SSU rDNA observations and SSU rRNA: rDNA ratios from samples collected at the same water column depths during peak water column stratification in July 2014.

Consistent with observed patterns in 2008, rDNA observations in 2014 showed Syndiniales group I and II OTUs were evenly distributed throughout the water column exhibiting highest relative abundance, 10 and 38\% respectively, in suboxic and anoxic water column conditions (Fig 9). Syndiniales group III OTUs distributed from surface (4\% relative abundance) to the suboxic boundary of the water column, while Syndiniales group V OTUs were primarily observed in dysoxic-suboxic water column conditions with a relative abundance $<0.1 \%$ (Fig 9). Choreotrichia OTUs were also found evenly distributed throughout the water column and showed higher relative abundance values $(\sim 6 \%)$ below the dysoxic boundary of the water column and highest abundance in anoxic waters (16\%) (Fig 9). Maxillopoda OTUs were found evenly distributed throughout the water column with a relative abundance $\sim 0.45 \%$, while Phaeocystis sp. OTUs were mostly distributed at dysoxic-suboxic water column conditions with a relative abundance $\sim 0.01 \%$ (Fig 9).

Potential activity of these OTUs was determined by calculating rRNA:rDNA ratios across depth intervals $(10,100,120,135,150,165$ and 185m) spanning the $\mathrm{O} 2$ concentration ranges from oxic to anoxic. In contrast with rDNA observations alone, rRNA observations can provide a robust proxy for past, present or emerging cellular activities (Blazewicz et al., 2013). Moreover, monitoring rRNA dynamics over time can inform hypotheses related to life strategies within communities (Barnard et al., 2013; Lepp and Schmidt, 1998). We selected OTUs affiliated with indicator OTUs showing significant correlations and considered values $\geq 1$ as potentially occurring interactions among likely active OTUs in the same depth interval. For instance, Syndiniales group II OTUs were active throughout the water column exhibiting the highest ratio (9.3) at 120m corresponding to the suboxic water column compartment (Fig 8). Similarly, Choreotrichia, Phaeocystis and Maxillopoda OTUs were found throughout the water column but exhibited the highest ratios in suboxic waters. For instance, Choreotrichia OTUs showed the highest ratio (7.1) at $135 \mathrm{~m}$, while Phaeocystis and Maxillopoda OTUs at 150m with ratio values equal to 14 and 8.6, respectively (Fig 8). Thus, it could be hypothesized that potential interactions among active OTUs could occur primarily at dysoxic-suboxic water column conditions between the $100 \mathrm{~m}$ and $150 \mathrm{~m}$. 


\section{DISCUSSION}

This study explores protistan diversity, and seasonal changes in abundance and distribution throughout the water column in Saanich Inlet, a seasonally anoxic fjord that serves as a wellcharacterized model ecosystem for examining how deoxygenation shapes microbial community population dynamics and interactions along defined redox gradients in the ocean. Our observations primarily focused on identifying statistically significant correlations that hint to potential interactions between the protistan parasite Syndiniales and potential host taxa. Proposed interactions were based on correlational edges between OTUs obtained from Bray-Curtis and Spearman's rank correlations and reinforced by determining potential activity from rRNA:rDNA ratios for these groups based on the assumption that potential parasitic interactions are most likely to occur at depths where the host is likely to be active/alive. Our results provide insights into the potential for parasitic interactions occurring under suboxic conditions during the summer stratification period with possible implications for carbon cycling and bloom termination dynamics.

Water column seasonal stratification and deep-water renewal restructures microbial eukaryotic communities that respond to changing $\mathrm{O} 2$ concentrations by altering their vertical distribution and activities (Duret et al., 2015; Jing et al., 2015; Parris et al., 2014). For instance, a previous study in Saanich Inlet highlighted seasonal differences in eukaryotic community structure correlated to water column $\mathrm{O} 2$ conditions (Orsi et al. 2012). Similarly, the use of time resolved SSU rDNA pyrotag observations over this seasonal cycle allowed us to observe changes in abundance and distribution patterns of the eukaryote community along defined water column $\mathrm{O} 2$ conditions. Although, the use of high-throughput sequencing data allowed detailed exploration of eukaryote diversity, we focused this analysis on Syndiniales, that comprise a diverse and abundant ( $43 \%$ during the summer stratification period) group of parasitic dinoflagellates that are responsive to water column stratification and deep-water renewal, and potentially play a significant role in shaping microbial biogeochemistry and ecology in diverse water column conditions. As an initial approach to select potential OTU pairs that likely showed predator-prey or parasitic interactions, we used ISA and co-occurrence correlation analyses in complementary manner. For instance, Syndiniales OTUs exhibited concurrent patterns with three likely host taxa i.e. Choreotrichia, Phaeocystis sp. and Maxillopoda and were selected based on 
statistical significance then further analyzed to provide baseline evidence for developing ecological hypotheses on predator-prey or parasitic interactions that may be relevant in stratified and $\mathrm{O} 2$ deficient marine environments.

Syndiniales interactions and the significance of peak stratification

The initial co-occurrence correlation analysis considered the eukaryotic community throughout 12-month period and identified five Syndiniales OTUs that have statistically significant correlations with other cosmopolitan eukaryotic taxa including Stramenopiles from the MAST clades, picomonads, and other Syndiniales (Supplementary Information Table S1). These taxa are small $(2-8 \mu \mathrm{m})$ and not previously documented in the literature as hosts of Syndiniales; however, spatial and temporal trends of these interactions reveal Syndiniales OTUs track the water column distribution of other microbial eukaryotes from surface water spring blooms to mid-waters as the inlet stratification strengthens in July (Supplementary Information Table S24). Syndiniales OTUs were abundant throughout the year, yet there was an absence of significant correlations detected in the 12-month analysis between Syndiniales and known host taxa. To reveal more ephemeral interactions between Syndiniales and common bloom-forming taxa (i.e. diatoms and dinoflagellates), we repeated our co-occurrence correlation analysis by targeting the period May through August, encompassing the peak stratification period.

Co-occurrence analysis of data collected during peak stratification revealed correlations among members of a diverse protistan community. Syndiniales OTUs were at the core of complex significant correlations with other protist taxa. The number of significant correlations for this period increased 10-fold and involved more diverse taxa, including several species of diatoms, the athecate dinoflagellate Gymnodinium, the bloom-forming haptophyte Phaeocystis, and heterotrophs, including several species of ciliates, stramenopiles, groups of small flagellates, and Metazoa. Many eukaryotic OTUs showed significant correlations with multiple Syndiniales OTUs (Supplementary Information Table S5), suggesting co-occurrence might drive host plasticity as a survival strategy that allows parasites to respond to host community shifts. Given the short-lived, free-swimming infective stage, we hypothesized co-occurrences between Syndiniales and other eukaryotes in the Saanich Inlet water column are more likely to reflect parasitism or predator-prey relationships than simple co-occurrences under specific water column conditions. Indicator analyses were compared with results from the co-occurrence 
correlation analysis to gain insights about taxa that were uniquely abundant during the spring and summer months and that showed significant correlation with Syndiniales over the same time period.

Based on these criteria, the distributions of three taxa were examined in detail:

Phaeocystis antarctica, a recurring marine haptophyte in Saanich Inlet (Orsi et al., 2012, 2018), which produced a bloom in 2008 providing an opportunity to investigate if parasitic dinoflagellates were involved in the termination of the bloom; a ciliate OTU affiliated to Choreotrichia, an indicator taxon during peak stratification in micro-oxic waters; and a metazoan copepod taxon, an indicator OTU during peak stratification. Ciliates, phytoplankton, and copepod Metazoa are all known to be potential hosts of Syndiniales (Dolan, 2013; Guillou et al., 2008; Massana et al., 2014). Observations of spatial and temporal distribution of OTUs affiliated to these taxa suggested that several types of parasitic Syndiniales could be potentially infecting the same host, and/or in the case of heterotrophic protistan taxa, that the heterotrophs may be potentially feeding on Syndiniales dinospores released from previously infected sinking cells. Nonetheless, we acknowledge the possibility that a fraction of these OTUs may not be involved in predator/prey or parasitic relationships and may simply co-occur as a community under specific environmental conditions. Furthermore, single-cell and microscopic analyses could be used in the future to verify specific interactions proposed by our observations in this study.

\section{Interactions with Choreotrichia}

Tintinnid ciliates belonging to the order Choreotrichida are ubiquitous in the marine plankton, particularly in coastal waters where their numbers are generally higher. Tintinnids play different roles in eukaryotic communities; they are preyed upon by planktonic zooplankton (Montagnes et al., 2010), as well as larger protist taxa (Dolan, 2013). Also, as nanoplankton consumers, they have an important role in bloom termination and/or as vectors for transferring bloom-associated toxins to higher trophic levels (Dolan, 2013). Furthermore, some tintinnid ciliates have been observed to serve as hosts for at least two different parasites, including Syndiniales, and even single cells may be co-infected (Dolan, 2013). In our study we observed ciliates belonging to the subclass Choreotrichia were most abundant between $75 \mathrm{~m}$ and $150 \mathrm{~m}$ in August 2008, and these OTUs were indicator OTUs for micro-oxic mid-waters during peak stratification. Correlation 
analysis results showed seven indicator Choreotrichia OTUs that were significantly correlated with Syndiniales groups (I-V) OTUs (Supplementary Information Table S3). However, the distribution of Syndiniales Group V OTUs most closely matched the distribution patterns of the OTUs affiliated with Choreotrichia throughout the water column between May and August (Fig 6). For example, in July 2008, the four Syndiniales groups and the ciliate subclass Choreotrichia were present at the surface $10 \mathrm{~m}$ and in micro-oxic waters between $85 \mathrm{~m}$ and $120 \mathrm{~m}$ (Fig. 6). while in August, only Syndiniales Group V matched Choreotrichia abundance and distribution patterns in the mid- and deep-water samples (Fig 6). Analysis of rRNA:rDNA ratios during peak stratification in 2014 indicated potential activity of Choreotrichia at 10m, 97m, 135m, and 165m depths in waters that ranged from oxic to anoxic. Syndiniales Group II was also active at these depths, which may suggest either parasitism or grazing could take place under different water column $\mathrm{O} 2$ conditions. Interestingly, rRNA:rDNA ratios for Syndiniales Group I, III, and V indicated cells were not likely active in the upper oxic layer and less active in the suboxic (not present or RNA:DNA ratio $<1$ ), which supports the hypothesis that Choreotrichia may be feeding on dinospores from these groups during peak stratification (Fig 9). The correlations between OTUs affiliated with Choreotrichia and the four known Syndiniales groups in Saanich Inlet indicated by the correlation analysis suggest complex interactions could occur that are associated with season water column dynamics. Further single-cell analyses are required to reveal the extent to which parasitism versus grazing is contributing to these detected potential interactions.

Interactions with Phaeocystis antarctica

Phaeocystis antarctica is a colony-forming haptophyte that contributes to primary production and dimethylsulfide formation (Kettle et al., 1999). The dimethylsulfide produced by bloom events can be oxidized to sulphuric acid in the atmosphere where it can function as nuclei for cloud condensation, impacting global albedo (Charlson et al., 1987). P. antarctica has a complex life cycle that includes colonial, bloom-forming aggregates, individual flagellates (microzoospores), and a recently discovered zygotic "benthic stage" (Gaebler-Schwarz et al., 2010). In our study, we observed OTUs affiliated with $P$. antarctica comprised $6 \%$ of the pyrotag library at $10 \mathrm{~m}$ depth in May 2008, consistent with a late spring surface bloom. Based on the depth of detection of $P$. antarctica pyrotags in subsequent months, cells appeared to sink through the water column and signatures of this taxon disappeared following deep-water renewal 
in October 2008 (Fig 7). Four and eight OTUs affiliated with Syndiniales groups I and II, respectively, were identified as having significant correlations with this haptophyte, with OTUs from these two groups of Syndiniales tracking signatures of $P$. antarctica through the water column during peak stratification. rRNA:rDNA ratio analysis indicated $P$. antarctica was not potentially active at $10 \mathrm{~m}$, however its highest potential activity was observed at 150 meters in July 2014 as also reflected in its abundance based on rDNA observations alone. This suggests an ephemerally active bloom followed by sinking of likely infected cells and "benthic stage" zygotes (Fig 9). Following renewal (which occurred on October 14, 2008; Ocean Networks Canada VENUS node http://www.oceannetworks.ca) the co-occurrence between Group I and II Syndiniales OTUs and $P$. antarctica was not detected, however those two groups of Syndiniales appeared to correlate with different putative hosts in the surface waters, i.e., OTUs affiliated with Pseudocalanus copepods and maxillopods. Parasites of P. antarctica blooms have not been previously identified; however, based on our co-occurrence correlation analyses we hypothesize this haptophyte could host several strains of Group I and II Syndiniales, and that these two groups of parasites locate and infect new hosts when $P$. antarctica is absent from the water column. Our observation hints at a testable hypothesis for future research is that parasitism of Phaeocystis blooms in Saanich Inlet could play a role in controlling the duration of phytoplankton blooms that play a major role in spring primary production, and that influence dimethylsulfide synthesis.

\section{Interactions with copepods}

Common taxonomic groups of mesozooplankton in Saanich Inlet include calanoid copepods, chaetognaths, euphausiids, cnidarians, and ctenophores. These taxa are critical for trophic transfer of carbon in marine food webs (e.g. (Jónasdóttir et al., 2015) as mesozooplankton are predators of phytoplankton, heterotrophic protists, and bacteria, as well as prey items for larger metazoans including fish. Zooplankton observed from a time series collected off southern Vancouver Island undergo annual variations in abundance, leading to changes in overall biomass and relative species composition that can further influence the pelagic food web (Beamish et al., 1997; Bertram et al., 2001; Hargreaves et al., 1994). Copepods typically found in shelf and slope waters off the coast of British Columbia have regionally specific ranges, driven primarily by water temperature, in addition to inter-annual shifts in species abundance. A few occurrences of 
dinoflagellate syndinian parasites from marine alveolate Group II (MALV II) infecting copepods and copepod eggs have been reported in the literature (Gómez et al., 2009; Kimmerer and McKinnon, 1990; Skovgaard et al., 2005); however, the overall ecological effects of these infections are not known. Our co-occurrence correlation analysis shows that Metazoa exhibit significant correlations with Syndiniales in Saanich Inlet. While the observed correlations may imply Metazoa may be feeding directly on dinospores or more likely, infected prey, these also suggest that Syndiniales OTUs could parasitize certain Metazoa. In our study, we observed OTUs affiliated with the genera Corycaeus and Oncaea were indicator OTUs for surface waters in May when prey taxa including Thlassiosira aestivalis, Chrysochromulina rotalis, Phaeocystis antarctica, Micromonas sp., and Florenciellales were abundant, suggesting higher metazoan grazing activity during peak phytoplankton growth. Additionally, these copepods showed significant correlations with Syndiniales from Groups I and II and, like the other taxa discussed, Syndiniales OTUs appears to track the grazers throughout the water column (Fig. 8). The corresponding rRNA:rDNA ratios for OTUs affiliated with the crustacean class Maxillopoda (copepods) indicated potential activity of this group of metazoans in the upper oxic layer during peak stratification in July 2014 (Fig. 9). Syndiniales Group II rRNA:rDNA ratios indicated potential activity in the water column at the same depths as the maxillopods, while Group I Syndiniales was likely inactive. The RNA-based analyses thus suggest Syndiniales Group I OTUs are likely sinking cells, while Group II could potentially have parasitic or prey interactions with members of Maxillopoda below the surface. Given the apparent significance of the interactions between metazoan groups known to play important roles in marine food webs, we consider it is important to understand the extent to which members of Syndiniales parasitize metazoans and the impact that this may have on carbon and nutrient turnover parasitize metazoans.

\section{CONCLUSION}

Our study used molecular time series observations to detect interactions between groups of primarily uncultured, parasitic dinoflagellates within the Syndiniales and other eukaryotes along defined oxygen gradients in Saanich Inlet. Co-occurrence correlation and indicator species analyses revealed the potential for significant interactions may occur between four known Syndiniales groups and different protistan and metazoan taxa including the stramenopile MAST 
clades not previously known to be parasitized by Syndiniales. We focused our analysis on several eukaryotic OTUs showing significant correlations with Syndiniales during peak stratification, including Phaeocystis antarctica, ciliates affiliated with Choreotrichia, and copepods. Our observations provide baseline understanding on the potential host range of the major parasitic Syndiniales groups that could infect key primary producing and heterotrophic populations along stratified water columns. Our results give insight into possible impacts these infections may have on population dynamics, extensible to nutrient cycling processes, during seasonal water column stratification in Saanich Inlet.

\section{CONFLICT OF INTEREST}

The authors declare that the research was conducted in the absence of any commercial or financial relationships that could be construed as a potential conflict of interest.

\section{ACKNOWLEDGMENTS}

We thank Captain Ken Brown and his crew for all their support aboard The RSV Strickland, as well as our sea-going technicians at UBC, Chris Payne and Larysa Pakhomova. We thank Rich Pawlowicz and the UBC Department of Earth, Ocean \& Atmospheric Sciences (EOAS) for the generous use of the EOAS CTD. We also thank past and present members of the Hallam lab, especially the many undergraduate trainees for their contributions to cruise preparation and clean up, water filtration, analytic chemistry and sequencing support. MTB was funded by Consejo Nacional de Ciencia y Tecnología (CONACyT) scholarship and the Tula Foundation funded Centre for Microbial Diversity and Evolution. This work was performed under the auspices of the G. Unger Vetlesen and Ambrose Monell Foundations, the Tula Foundation-funded Centre for Microbial Diversity and Evolution, the Natural Sciences and Engineering Research Council of Canada, Genome British Columbia, the Canada Foundation for Innovation, and the Canadian Institute for Advanced Research through grants awarded to S.J.H.

\section{REFERENCES}

Allers, E., Wright, J.J., Konwar, K.M., Howes, C.G., Beneze, E., Hallam, S.J., and Sullivan, M.B., 2013. Diversity and population structure of Marine Group A bacteria in the Northeast subarctic Pacific Ocean. The ISME Journal, 7, pp.256-268.

Altschul, S.F., Gish, W., Miller, W., Myers, E.W., and Lipman, D.J., 1990. Basic local alignment search tool. Journal of Molecular Biology, 215, pp.403-410. 
Arístegui, J., Gasol, J.M., Duarte, C.M., and Herndld, G.J., 2009. Microbial oceanography of the dark ocean's pelagic realm. Limnology and Oceanography, 54, pp.1501-1529.

Azam, F., Fenchel, T., Field, J.G., Gray, J.S., Meyer-Reil, L.A., and Thingstad, F., 1983. The ecological role of water-column microbes in the sea. Marine Ecology Progress Series, 10, pp.257-263.

Bai, X., Adolf, J.E., Bachvaroff, T., Place, A.R., and Coats, D.W., 2007. The interplay between host toxins and parasitism by Amoebophrya. Harmful Algae, 6, pp.670-678.

Barnard, R.L., Osborne, C.A., and Firestone, M.K., 2013. Responses of soil bacterial and fungal communities to extreme desiccation and rewetting. The ISME journal, 7, pp.2229-2241.

Beamish, R.J., Neville, C.M., and Cass, A.J., 1997. Production of Fraser River sockeye salmon (Oncorhynchus nerka) in relation to decadal-scale changes in the climate and the ocean. Canadian Journal of Fisheries and Aquatic Sciences, 54, pp.543-554.

Bertram, D.F., Mackas, D.L., and McKinnell, S.M., 2001. The seasonal cycle revisited: interannual variation and ecosystem consequences. Progress in Oceanography, 49, pp.283-307.

Blazewicz, S.J., Barnard, R.L., Daly, R.A., and Firestone, M.K., 2013. Evaluating rRNA as an indicator of microbial activity in environmental communities: limitations and uses. The ISME Journal, 7, pp.2061-2068.

Bråte, J., Krabberød, A.K., Dolven, J.K., Ose, R.F., Kristensen, T., Bjørklund, K.R., and Shalchian-Tabrizi, K., 2012. Radiolaria Associated with Large Diversity of Marine Alveolates. Protist, 163, pp.767-777.

Cachon, J., 1964 Contribution a l'etude des peridiniens parasites. Cytologie, cycles evolutifs. Annales des Sciences Naturelles -Zoologie et Biologie Animale, 6, pp.1-158.

Caporaso, J.G., Kuczynski, J., Stombaugh, J., Bittinger, K., Bushman, F.D., Costello, E.K., Fierer, N., Pena, A.G., Goodrich, J.K., Gordon, J.I., Huttley, G.A., Kelley, S.T., Knights, D., Koenig, J.E., Ley, R.E., Lozupone, C.A., McDonald, D., Muegge, B.D., Pirrung, M., Reeder, J., Sevinsky, J.R., Turnbaugh, P.J., Walters, W.A., Widmann, J., Yatsunenko, T., Zaneveld, J., and Knight, R., 2010. QIIME allows analysis of high-throughput community sequencing data. Nature Methods, 7, pp.335-336.

Chambouvet, A., Alves-de-Souza, C., Cueff, V., Marie, D., Karpov, S., and Guillou, L., 2011. Interplay between the parasite Amoebophrya sp. (Alveolata) and the cyst formation of the red tide dinoflagellate Scrippsiella trochoidea. Protist, 162, pp.637-649.

Chambouvet, A., Morin, P., Marie, D., and Guillou, L., 2008. Control of toxic marine dinoflagellate blooms by serial parasitic killers. Science, 322, pp.1254-1257. 
Charlson, R.J., Lovelock, J.E., Andreae, M.O., and Warren, S.G., 1987. Oceanic phytoplankton, atmospheric sulphur, cloud albedo and climate. Nature, 326, pp.655-661.

Choi, C.J., Brosnahan, M.L., Sehein, T.R., Anderson, D.M., and Erdner, D.L., 2017. Insights into the loss factors of phytoplankton blooms: The role of cell mortality in the decline of two inshore Alexandrium blooms. Limnology and Oceanography, 62, pp.1742-1753.

Chow, C.-E.T., Kim, D.Y., Sachdeva, R., Caron, D.A., and Fuhrman, J.A., 2014. Top-down controls on bacterial community structure: microbial network analysis of bacteria, T4-like viruses and protists. The ISME Journal, 8, pp.816-829.

Coats, D.W., and Heisler, J.J., 1989. Spatial and temporal occurrence of the parasitic dinoflagellate Dubusquella cachoni and its tintinnine host Eutintinnus pectinis in Chesapeake Bay. Marine Biology, 101, pp.401-409.

Coats, D.W., Bachvaroff, T.R., and Delwiche, C.F., 2012. Revision of the family Duboscquellidae with a description of Euduboscquella crenulata n. gen., n. sp. (Dinoflagellata, Syndinea), an intracellular parasite of the cilliate Favella panamensis Kofoid \& Campbell, 1929. Journal of Eukaryotic Microbiology, 59, pp.1-11.

Coats, D.W., Bockstahler, K.R., Berg, G.M., and Sniezek, J.H., 1994. Dinoflagellate infections of Favella panamensis from two North American estuaries. Marine Biology, 119, pp.105-113.

Cuevas, L.A. and Morales, C.E., 2006. Nanoheterotroph grazing on bacteria and cyanobacteria in oxic and suboxic waters in coastal upwelling areas off northern Chile. Journal of Plankton Research, 28, pp.385-397.

De Caceres, M. and Legendre, P., 2009. Associations between species and groups of sites: indices and statistical inference. Ecology. http://sites.google.com/site/miqueldecaceres/.

de Vargas, C., Audic, S., Henry, N., Decelle, J., Mahe, F., Logares, R., Lara, E., Berney, C., Bescot, N.L., Probert, I., Carmichael, M., Poulain, J., Romac, S., Colin, S., Aury, J.-M., Bittner, L., Chaffron, S., Dunthorn, M., Engelen, S., Flegontova, O., Guidi, L., Horak, A., Jaillon, O., Lima-Mendez, G., Lukes, J., Malviya, S., Morard, R., Mulot, M., Scalco, E., Siano, R., Vincent, F., Zingone, A., Dimier, C., Picheral, M., Searson, S., Kandels-Lewis, S., Coordinators, T.O., Acinas, S.G., Bork, P., Bowler, C., Gorsky, G., Grimsley, N., Hingamp, P., Iudicone, D., Not, F., Ogata, H., Pesant, S., Raes, J., Sieracki, M.E., Speich, S., Stemmann, L., Sunagawa, S., Weissenbach, J., Wincker, P., and Karsenti, E., 2015. Eukaryotic planktonic diversity in the sunlit ocean. Science, 348(6237).

Dolan, J.R., Montagnes, D.J.S., Agathgam S., Coats, D.W., and Stoecker, D.K. eds., 2013. The Biology and Ecology of Tintinnid Ciliates, Models for Marine Plankton. Wiley-Blackwell, Hoboken, NJ, USA. 
Duret, M.T., Pachiadaki, M.G., Stewart, F.J., Sarode, N., Christaki, U., Monchy, S., Srivastava, A., and Edgcomb, V.P., 2015. Size-fractionated diversity of eukaryotic microbial communities in the Eastern Tropical North Pacific oxygen minimum zone. FEMS Microbiology Ecology, 91(5).

Edgcomb, V., Orsi, W., Bunge, J., Jeon, S., Christen, R., Leslin, C., Holder, M., Taylor, G.T., Suarez, P., Varela, R., and Epstein, S., 2011. Protistan microbial observatory in the Cariaco Basin, Caribbean. I. Pyrosequencing vs Sanger insights into species richness. The ISME Journal, 5, pp.1344-1356.

Edgcomb, V.P., 2016. Marine protist associations and environmental impacts across trophic levels in the twilight zone and below. Current Opinion in Microbiology, 31, pp.169-175.

Escribano, R., Hidalgo, P., and Krautz, C., 2009. Zooplankton associated with the oxygen minimum zone system in the northern upwelling region of Chile during March 2000. Deep Sea Research Part II: Topical Studies in Oceanography, 56, pp.1083-1094.

Faust, K., Sathirapongsasuti, J.F., Izard, J., Segata, N., Gevers, D., Raes, J., and Huttenhower, C., 2012. Microbial Co-occurrence Relationships in the Human Microbiome. PLoS Computational Biology, 8, e1002606.

Gaebler-Schwarz, S., Davidson, A., Assmy, P., Chen, J., Henjes, J., Nöthig, E.M., Lunau, M., and Medlin, L.K., 2010. A new cell stage in the haploid-diploid life cycle of the colony-forming haptophyte Phaeocystis antarctica and its ecological implications. Journal of Phycology, 46, pp.1006-1016.

Gómez, F., Moreira, D., and López-García, P., 2009. Life cycle and molecular phylogeny of the dinoflagellates Chytriodinium and Dissodinium, ectoparasites of copepod eggs. European journal of protistology, 45, pp.260-270.

Guillou, L., Viprey, M., Chambouvet, A., Welsh, R., Kirkham, A., Massana, R., Scanlan, D.J., and Worden, A., 2008. Widespread occurrence and genetic diversity of marine parasitoids belonging to Syndiniales (Alveolata). Environmental Microbiology, 10, pp.3349-3365.

Hargreaves, N., Ware, D., and McFarlane, G., 1994. Return of Pacific sardine (Sardinops sagax) to the British Columbia coast in 1992. Canadian Journal of Fisheries and Aquatic Sciences, 51, pp.460-463.

Hawley, A.K., Torres-Beltrán, M., Zaikova, E., Walsh, D.A., Mueller, A., Scofield, M., Kheirandish, S., Payne, C., Pakhomova, L., Bhatia, M., Shevchuk, O., Gies, E.A., Fairley, D., Malfatti, S.A., Norbeck, A.D., Brewer, H.M., Pasa-Tolic, L., del Rio, T.G., Suttle, C.A., Tringe, S., and Hallam, S.J., 2017. A compendium of multi-omic sequence information from the Saanich Inlet water column. Scientific Data, 4, 170160.

Jephcott, T.G., Alves-de-Souza, C., Gleason, F.H., van Ogtrop, F.F., Sime-Ngando, T., Karpov, S.A., and Guillou, L., 2016. Ecological impacts of parasitic chytrids, syndiniales and perkinsids on populations of marine photosynthetic dinoflagellates. Fungal Ecology, 19, pp.47-58. 
Jing, H., Rocke, E., Kong, L., Xia, X., Liu, H., and Landry, M.R., 2015. Protist communities in a marine oxygen minimum zone off Costa Rica by 454 pyrosequencing. Biogeosciences Discussions, 12(16), pp.13483-13509.

Jónasdóttir, S.H., Visser, A.W., Richardson, K., and Heath, M.R., 2015. Seasonal copepod lipid pump promotes carbon sequestration in the deep North Atlantic. Proceedings of the National Academy of Sciences, 112, pp.12122-12126.

Kettle, A., Andreae, M., Amouroux, D., Andreae, T., Bates, T., Berresheim, H., Bingemer, H., Boniforti, R., Curran, M., and DiTullio, G., 1999. A global database of sea surface dimethylsulfide (DMS) measurements and a procedure to predict sea surface DMS as a function of latitude, longitude, and month. Global Biogeochemical Cycles, 13, pp.399-444.

Kimmerer, W. and McKinnon, A., 1990. High mortality in a copepod population caused by a parasitic dinoflagellate. Marine Biology, 107, pp.449-452.

Legendre, P. and Gallagher, E.D., 2001. Ecologically meaningful transformations for ordination of species data. Oecologia, 129, pp.271-280.

Lepp, P.W. and Schmidt, T.M., 1998. Nucleic acid content of Synechococcus spp. during growth in continuous light and light/dark cycles. Archives of Microbiology, 170, pp.201-207.

Lin, X., Scranton, M.I., Varela, R., Chistoserdov, A., and Taylor, G.T., 2007. Compositional responses of bacterial communities to redox gradients and grazing in the anoxic Cariaco Basin. Aquatic Microbial Ecology, 47, pp.57-72.

Lopez-Garcia, P., Rodriguez-Valera, F., Pedros-Alio, C., and Moreira, D., 2001. Unexpected diversity of small eukaryotes in deep-sea Antarctic plankton. Nature, 409, pp.603-607.

Massana, R., Balagué, V., Guillou, L., and Pedros-Alio, C., 2004a. Picoeukaryotic diversity in an oligotrophic coastal site studied by molecular and culturing approaches. FEMS Microbiology Ecology, 50, pp.231-243.

Massana, R., Castresana, J., Balagué, V., Guillou, L., Romari, K., Groisillier, A., Valentin, K., and Pedrós-Alió, C., 2004b. Phylogenetic and ecological analysis of novel marine stramenopiles. Applied and Environmental Microbiology, 70, pp.3528-3534.

Massana, R., del Campo, J., Sieracki, M.E., Audic, S., and Logares, R., 2014. Exploring the uncultured microeukaryote majority in the oceans: reevaluation of ribogroups within stramenopiles. The ISME Journal, 8, pp.854-866.

Montagnes, D.J.S., Dower, J.F., and Figueiredo, G.M., 2010. The ProtozooplanktonIchthyoplankton Trophic Link: An Overlooked Aspect of Aquatic Food Webs. Journal of Eukaryotic Microbiology, 57, pp.223-228. 
Moon-van der Staay, S.Y., De Wachter, R., and Vaulot, D., 2001. Oceanic 18S rDNA sequences from picoplankton reveal unsuspected eukaryotic diversity. Nature, 409, pp.607-610.

Not, F., Campo, J.d., Balague, V., Vargas, C.d., and Massana, R., 2009. New insights into the diversity of marine picoeukaryotes. PLoS One, 4(9), p.e7143.

Not, F., Valentin, K., Romari, K., Lovejoy, C., Massana, R., Töbe, K., Vaulot, D., and Medlin, L.K., 2007. Picobiliphytes: a marine picoplanktonic algal group with unknown affinities to other eukaryotes. Science, 315, pp.253-255.

Orsi, W., Song, Y.C., Hallam, S., and Edgcomb, V., 2012. Effect of oxygen minimum zone formation on communities of marine protists. The ISME Journal, 6, pp.1586-1601.

Orsi, W.D., Wilken, S., Del Campo, J., Heger, T., James, E., Richards, T.A., Keeling, P.J., Worden, A.Z. and Santoro, A.E., 2018. Identifying protist consumers of photosynthetic picoeukaryotes in the surface ocean using stable isotope probing. Environmental microbiology, 20(2), pp.815-827.

Parris, D.J., Ganesh, S., Edgcomb, V.P., DeLong, E.F., and Stewart, F.J., 2014. Microbial eukaryote diversity in the marine oxygen minimum zone off northern Chile. Frontiers in Microbiology, 5, pp.543.

Paulmier, A. and Ruiz-Pino, D., 2009. Oxygen minimum zones (OMZs) in the modern ocean. Progress in Oceanography, 80, pp.113-128.

Pedrós-Alió, C., Calderón-Paz, J.I., and Gasol, J.M., 2000. Comparative analysis shows that bacterivory, not viral lysis, controls the abundance of heterotrophic prokaryotic plankton. FEMS Microbiology Ecology, 32, pp.157-165.

Pruesse, E., Quast, C., Knittel, K., Fuchs, B.M., Ludwig, W., Peplies, J., and Glöckner, F.O., 2007. SILVA: a comprehensive online resource for quality checked and aligned ribosomal RNA sequence data compatible with ARB. Nucleic Acids Research, 35, pp.7188-7196.

RCoreTeam, 2013. R: A language and environment for statistical computing. R Foundation for Statistical Computing, Vienna, Austria. URL http://www.R-project.org/.

Richards, T.A.and Bass, D., 2005. Molecular screening of free-living microbial eukaryotes: diversity and distribution using a meta-analysis. Current Opinion in Microbiology, 8, pp.240252.

Romari, K. and Vaulot, D., 2004. Composition and temporal variability of picoeukaryote communities at a coastal site of the English Channel from 18S rDNA sequences. Limnology and Oceanography, 49, pp.784-798.

Schmidtko, S., Stramma, L., and Visbeck, M., 2017. Decline in global oceanic oxygen content during the past five decades. Nature, 542, pp.335-339. 
Shannon, P., Markiel, A., Ozier, O., Baliga, N.S., Wang, J.T., Ramage, D., Amin, N., Schwikowski, B., and Ideker, T., 2003. Cytoscape: A Software Environment for Integrated Models of Biomolecular Interaction Networks. Genome Research, 13, pp.2498-2504.

Shi, Y., Tyson, G.W., and DeLong, E.F., 2009. Metatranscriptomics reveals unique microbial small RNAs in the ocean's water column. Nature, 459, pp.266-269.

Skovgaard, A., Massana, R., Balague, V., and Saiz, E., 2005. Phylogenetic position of the copepod-infesting parasite Syndinium turbo (Dinoflagellata, Syndinea). Protist, 156, pp.413-423.

Stewart, F.J., Ottesen, E.A., and DeLong, E.F., 2010. Development and quantitative analyses of a universal rRNA-subtraction protocol for microbial metatranscriptomics. The ISME Journal, 4, pp.896-907.

Stramma, L., Johnson, G.C., Sprintall, J., and Mohrholz, V., 2008. Expanding Oxygen-Minimum Zones in the Tropical Oceans. Science, 320, pp.655.

Suttle, C.A., 2005. Viruses in the sea. Nature, 437, pp.356-361.

Suzuki, R. and Shimodaira, H., 2015. pvclust: Hierarchical Clustering with P-Values via Multiscale Bootstrap Resampling. R package version 2.0-0. http://CRAN.Rproject.org/package $=$ pvclust.

Taylor, G.T., Karl, D.L., and Pace, M.L., 1986. Impact of bacteria and zooflagellates on the composition of sinking particles: An in-situ experiment. Marine Ecology Progress Series, 29, pp.141-155.

Torres-Beltrán, M., Hawley, A., Capelle, D., Bhatia, M., Durno, E., Tortell, P., and Hallam, S., 2016. Methanotrophic community dynamics in a seasonally anoxic fjord: Saanich Inlet, British Columbia. Frontiers in Marine Science, 3, pp.268.

Torres-Beltrán, M., Hawley, A.K., Capelle, D., Zaikova, E., Walsh, D.A., Mueller, A., Scofield, M., Payne, C., Pakhomova, L., Kheirandish, S., Finke, J., Bhatia, M., Shevchuk, O., Gies, E.A., Fairley, D., Michiels, C., Suttle, C.A., Whitney, F., Crowe, S.A., Tortell, P.D., and Hallam, S.J., 2017. A compendium of geochemical information from the Saanich Inlet water column.

Scientific Data, 4, pp.170159.

Ulloa, O. and Pantoja, S., 2009. The oxygen minimum zone of the eastern South Pacific. Deep Sea Research Part II: Topical Studies in Oceanography, 56, pp.987-991.

Velo-Suárez, L., Brosnahan, M.L., Anderson, D.M., and McGillicuddy, D.J., Jr., 2013. A quantitative assessment of the role of the parasite Amoebophrya in the termination of Alexandrium fundyense blooms within a small coastal embayment. PLOS One, 8, e81150. 
Wishner, K.F., Gowing, M.M., and Gelfman, C., 1998. Mesozooplankton biomass in the upper $1000 \mathrm{~m}$ in the Arabian Sea: overall seasonal and geographic patterns, and relationship to oxygen gradients. Deep Sea Research Part II: Topical Studies in Oceanography, 45, pp.2405-2432.

Wishner, K.F., Outram, D.M., Seibel, B.A., Daly, K.L., and Williams, R.L., 2013. Zooplankton in the eastern tropical north Pacific: Boundary effects of oxygen minimum zone expansion. Deep Sea Research Part I: Oceanographic Research Papers, 79, pp.122-140.

Wright, J.J., Konwar, K.M., and Hallam, S.J., 2012. Microbial ecology of expanding oxygen minimum zones. Nature Reviews Microbiology, 10, pp.381-394.

Zaikova, E., Walsh, D.A., Stilwell, C.P., Mohn, W.W., Tortell, P.D., and Hallam, S.J., 2010. Microbial community dynamics in a seasonally anoxic fjord: Saanich Inlet, British Columbia. Environmental Microbiology, 12, pp.172-191. 


\section{FIGURES}
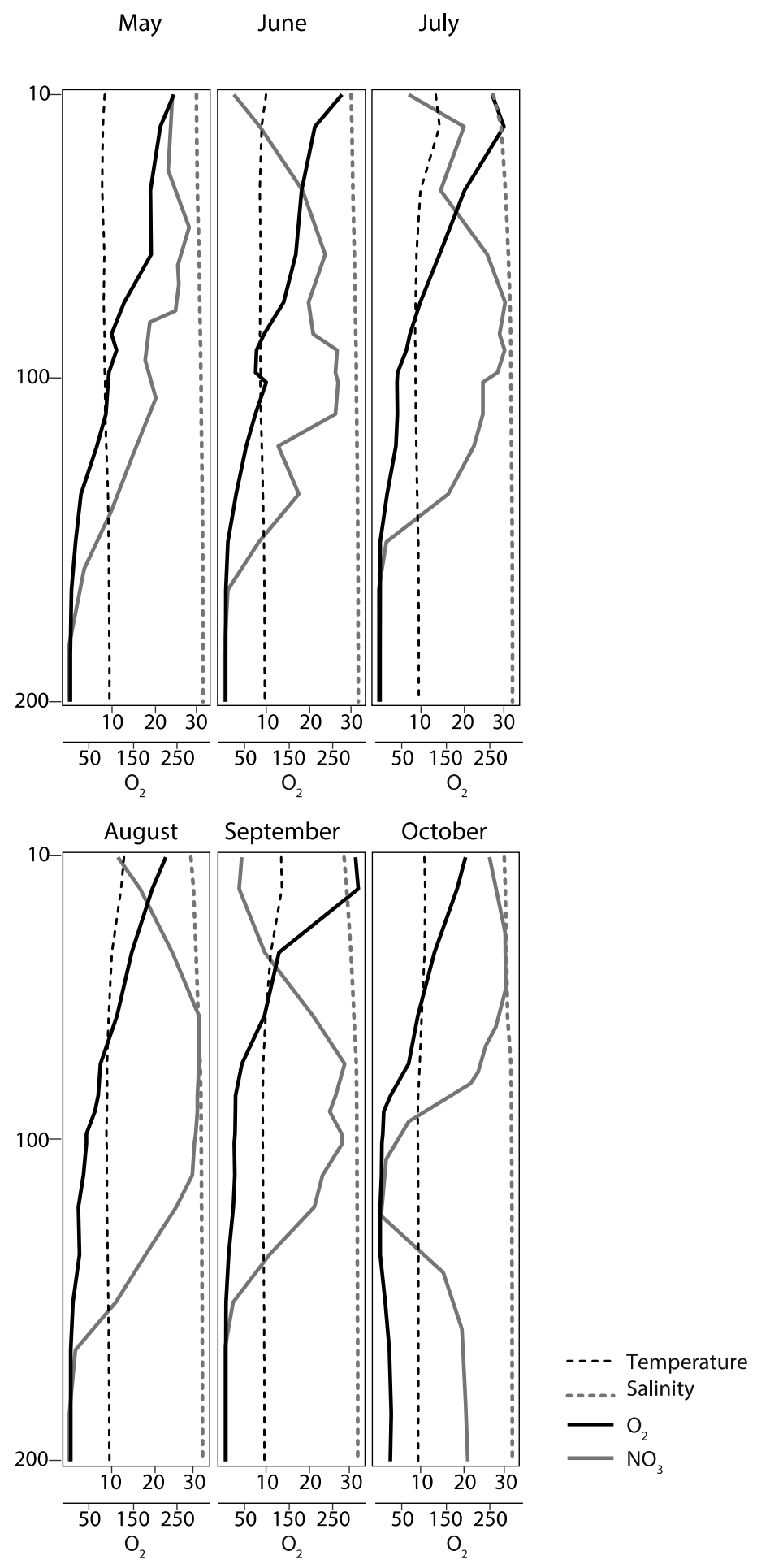

Figure 1. CTD and chemical data shown as monthly panels for temperature $\left({ }^{\circ} \mathrm{C}\right)$, Salinity (ppt), Oxygen $(\mu \mathrm{M})$ and nitrate $(\mu \mathrm{M})$ along the depth profile for samples taken from May to September 2008 at Station S3 in Saanich Inlet. 
A

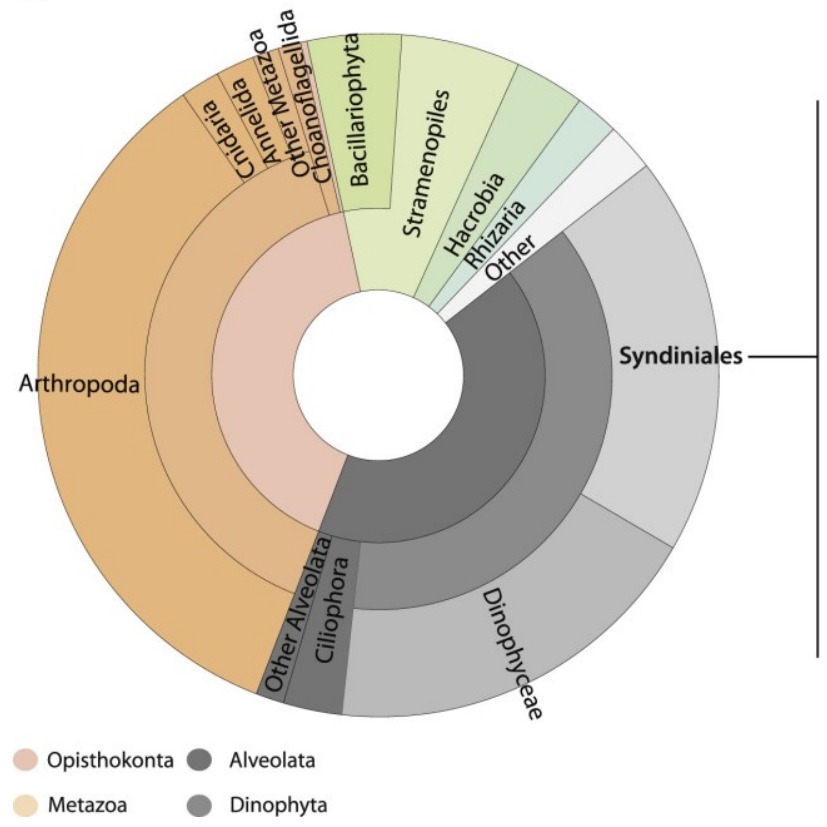

B

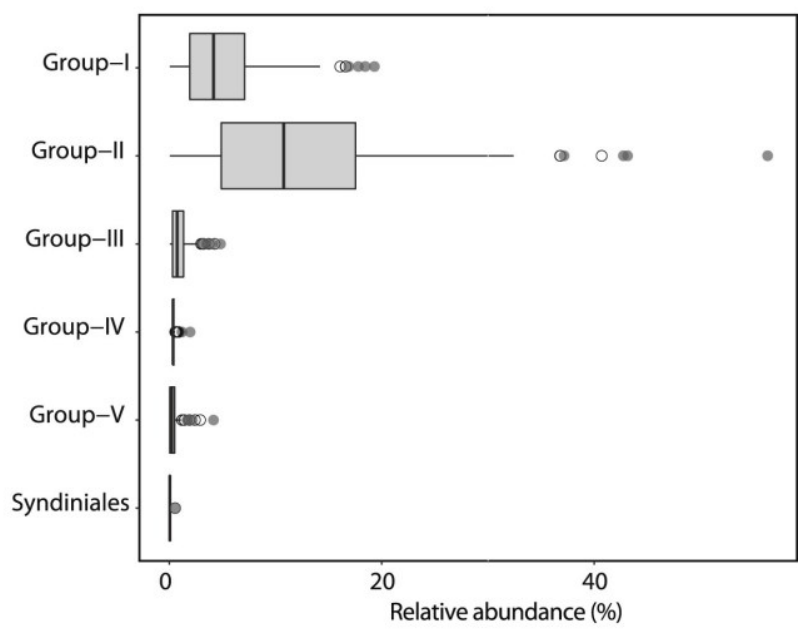

Figure 2. A) Krona chart showing the taxonomic composition of eukaryotic OTUs found in the rDNA pyrotag datasets from May 2008 to April 2009. Layers represent hierarchical taxonomy from the upper (centre) to the lowest (outer) taxonomic level. B) Relative abundance for Syndiniales OTUs divided by taxonomic groups found in pyrotag dataset. The size of each box represents the average of relative abundance (\%) calculated from the total number of eukaryotic reads throughout the water column over this period. Extended dashed lines (whiskers) represent at the base the lower and upper quartiles $(25 \%$ and $75 \%)$ and at the end the minimum and maximum values encountered. The middle line represents the median 
A

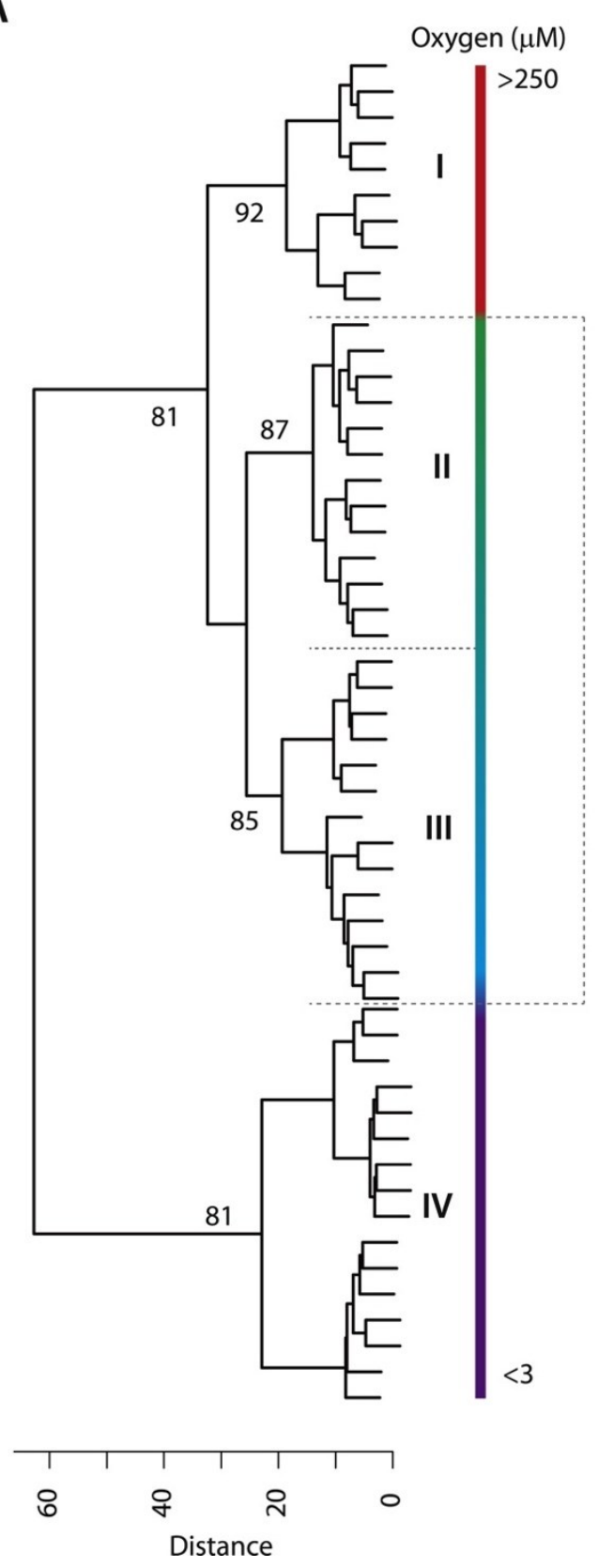

B

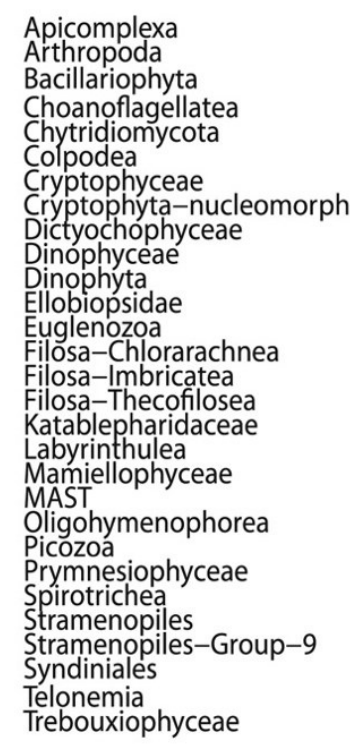

C

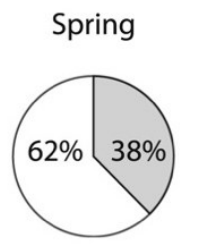

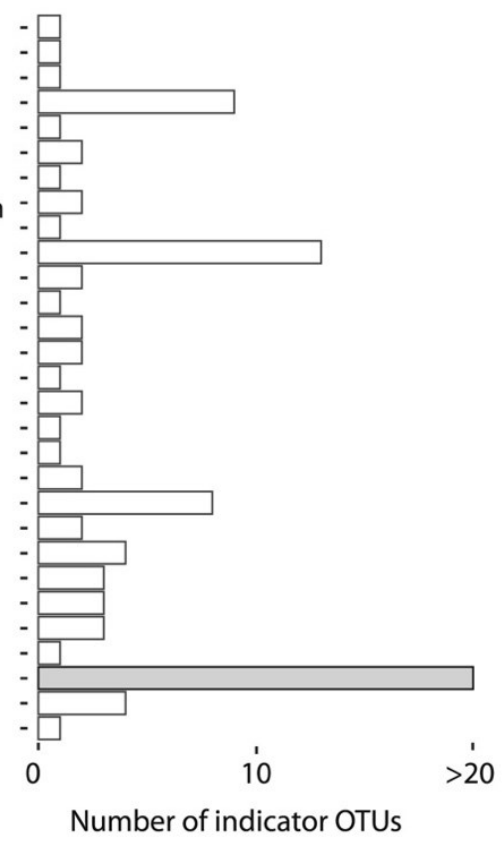

Summer (Stratification)

Fall (Renewal)
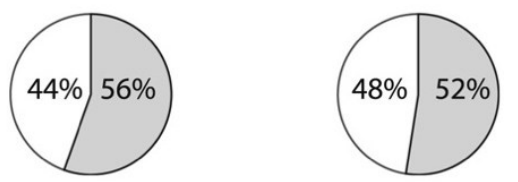

Figure 3. A) Hierarchical clustering of eukaryotic pyrotag data during peak stratification based on Manhattan distance. Clusters are delimited by $\mathrm{O} 2$ concentration range represented by number from I to IV: oxic = I (red), dysoxic- suboxic = II and III (green and blue, respectively), and anoxic $=$ IV (purple). Bootstrap values (1000 iterations) are shown in gray. B) Indicator OTUs (Indicator value $0.6, p=0.05, \alpha \leq 0.01$ ) for suboxic-dysoxic water column conditions during peak stratification. Bars depict the total number of OTUs for each taxonomic group observed. Total

Syndiniales indicator OTUs $(n=54)$ are shown in dark gray. C) Distribution of Syndiniales indicator OTUs over three different water column periods in the inlet (spring, summer stratification and fall deep-water renewal). Pie charts depict the percentage of Syndiniales OTUs (dark gray) out of the total number of indicator OTUs for each period. 


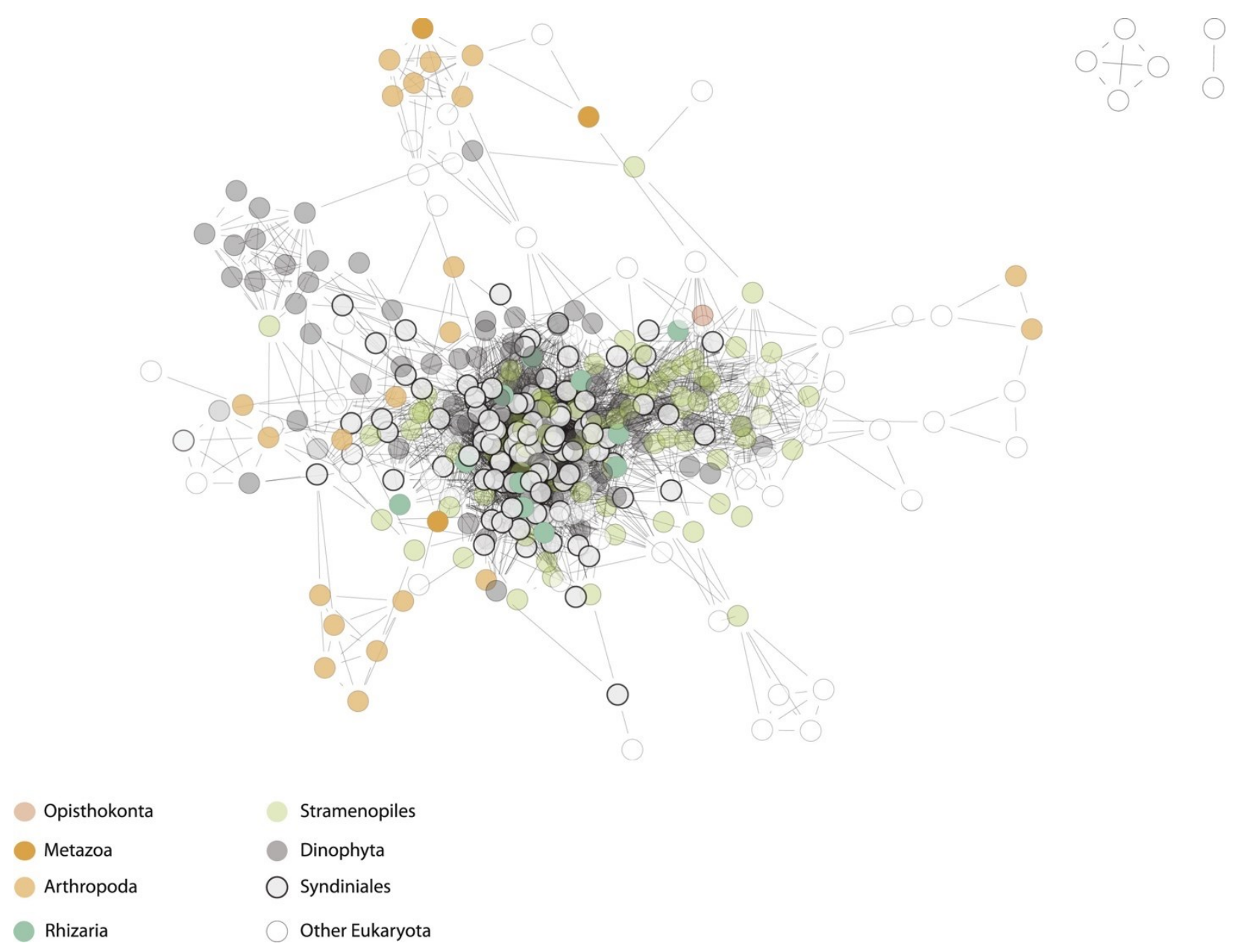

Figure 4. Co-occurrence network derived from Bray-Curtis and Spearman correlation measures on rDNA pyrotag protist data from May-August 2008. Nodes depict OTUs and edges cooccurrence correlations. Syndiniales OTUs are highlighted in gray and other protist OTUs coloured as indicated in color key. 
A
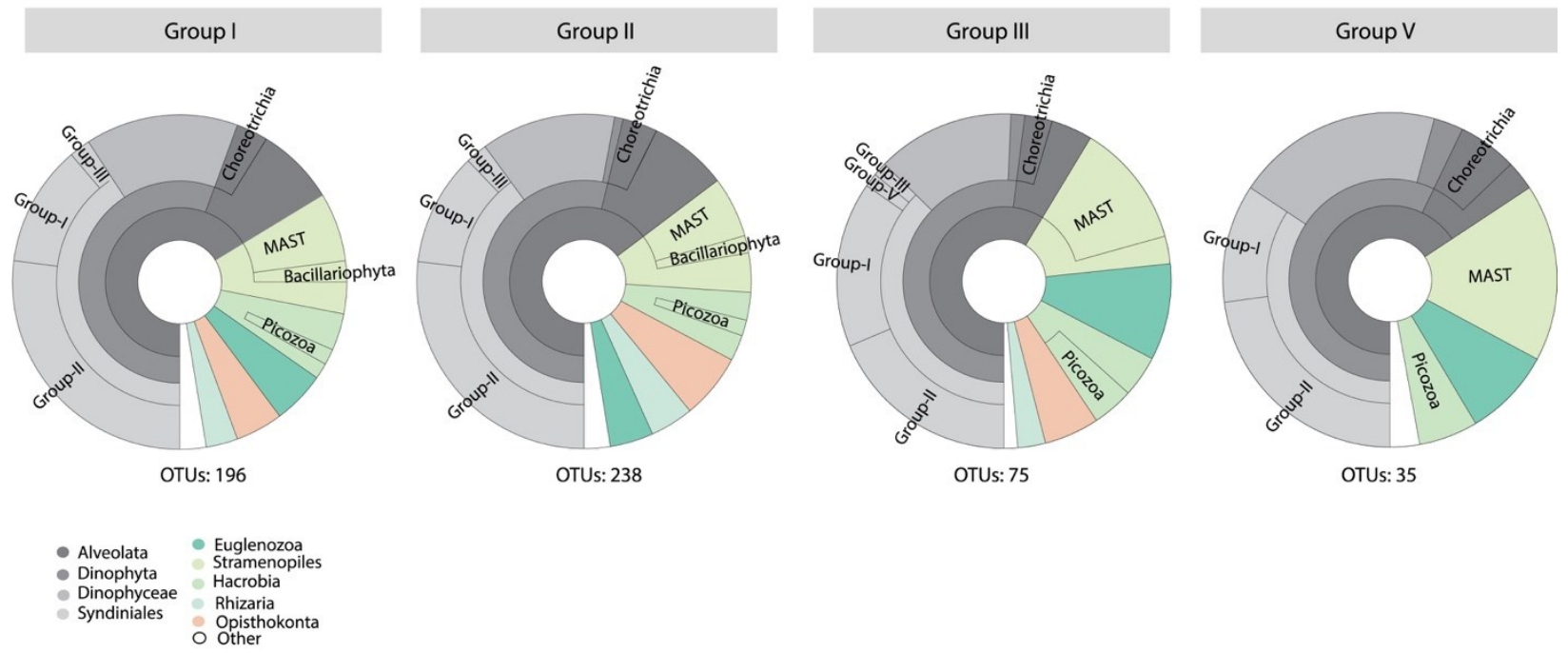

\section{B}

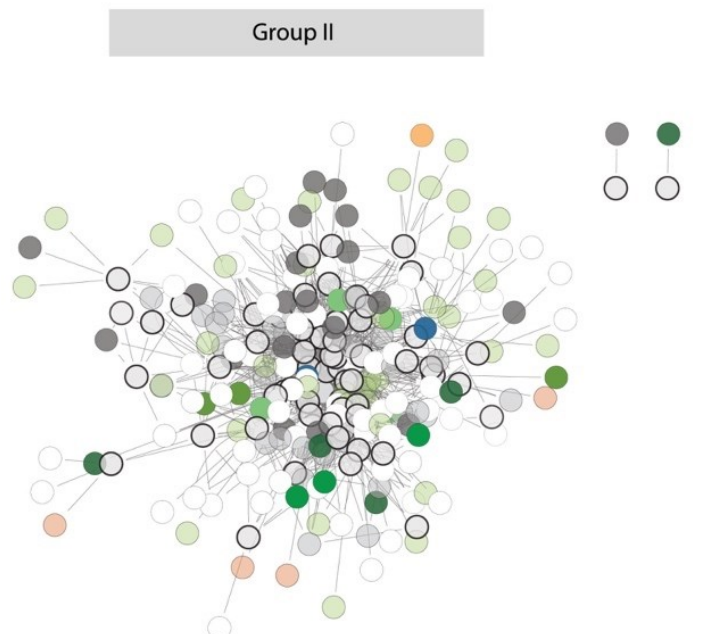

Group I
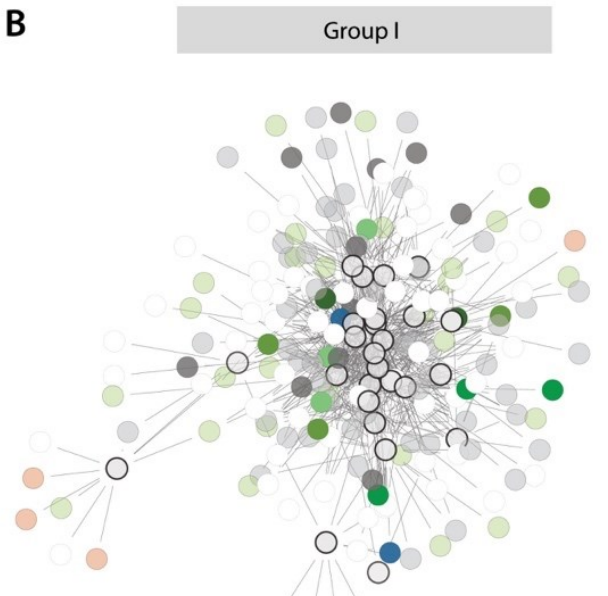

Opisthokonta
Metazoa
Arthropoda
Rhizaria

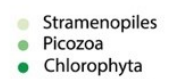

- Chlorophyta

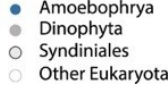

Figure 5. A) Eukaryotic taxa diversity associated with each Syndiniales group. Krona charts depict total unique OTUs with significant interactions for each Syndiniales group (I, II, III and

V). Layers represent hierarchical taxonomy from the upper (centre) to the lowest (outer) taxonomic level. Total number of unique OTUs is indicated at the bottom of each chart. B) Cooccurrence network derived from Bray-Curtis and Spearman correlation measures on Sydiniales Group I and II derived from rDNA pyrotag protist data from May-August 2008. Nodes depict OTUs and edges co-occurrence correlations. Syndiniales OTUs are highlighted in gray and other protist OTUs coloured as indicated in color key. 


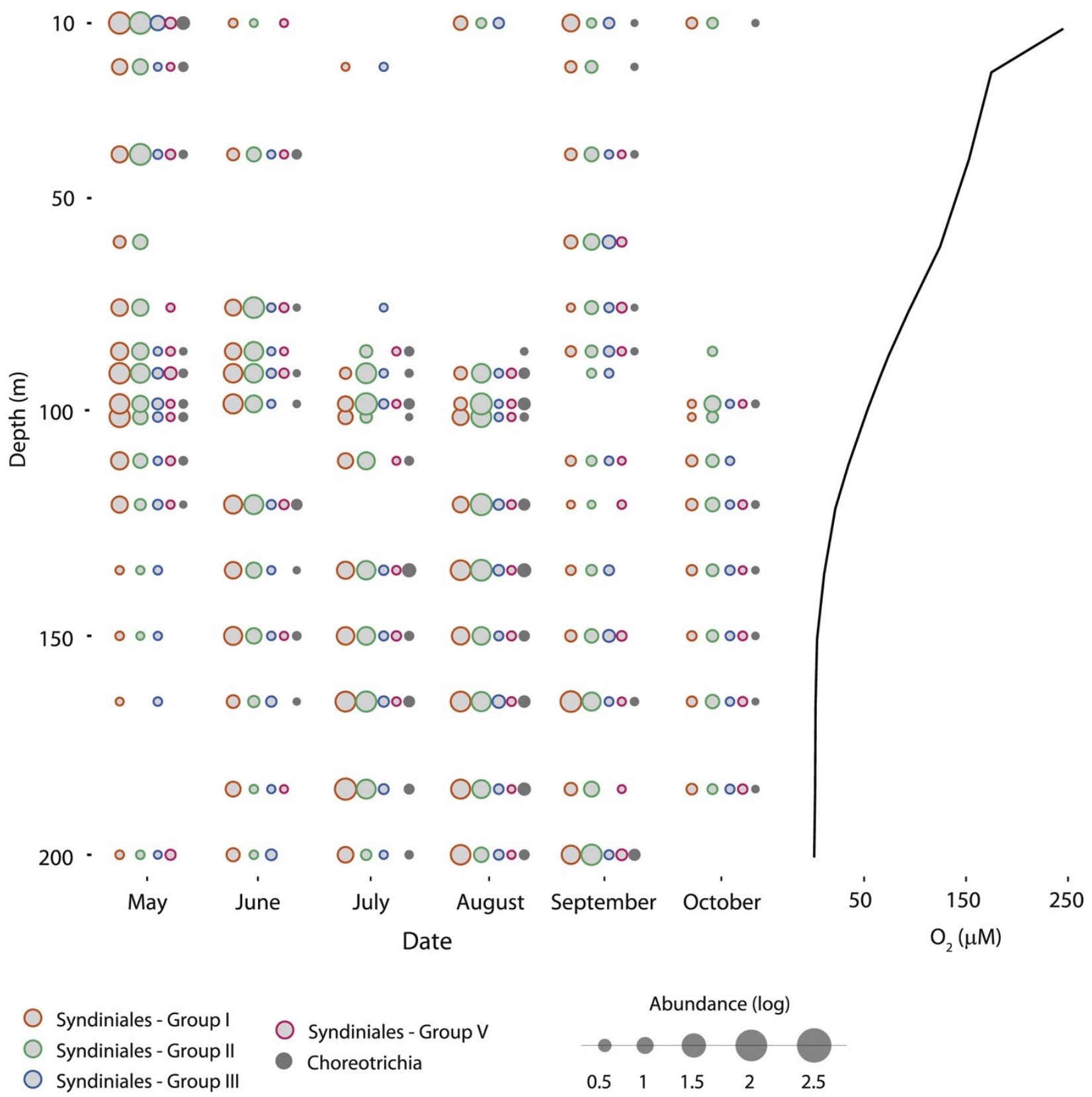

Figure 6. Vertical distribution and abundance of Choreotrichia and Syndiniales OTUs through the water column from May-October 2008. Dots size depicts OTUs relative abundance in logarithmic scale. 


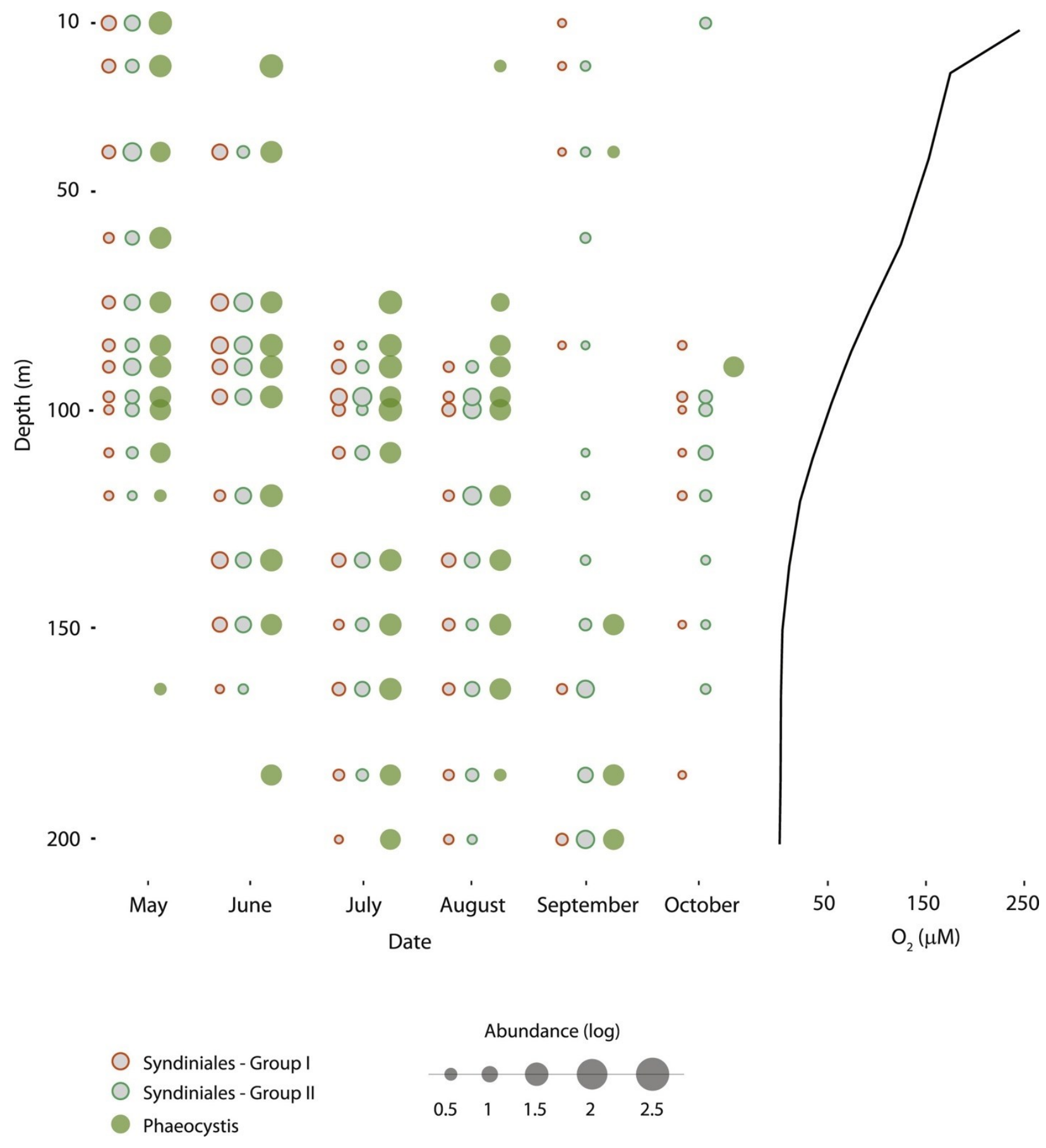

Figure 7. Vertical distribution and abundance of Phaeocystis antarctica and Syndiniales OTUs through the water column from May-October 2008. Bars depict OTUs relative abundance in logarithmic scale. 


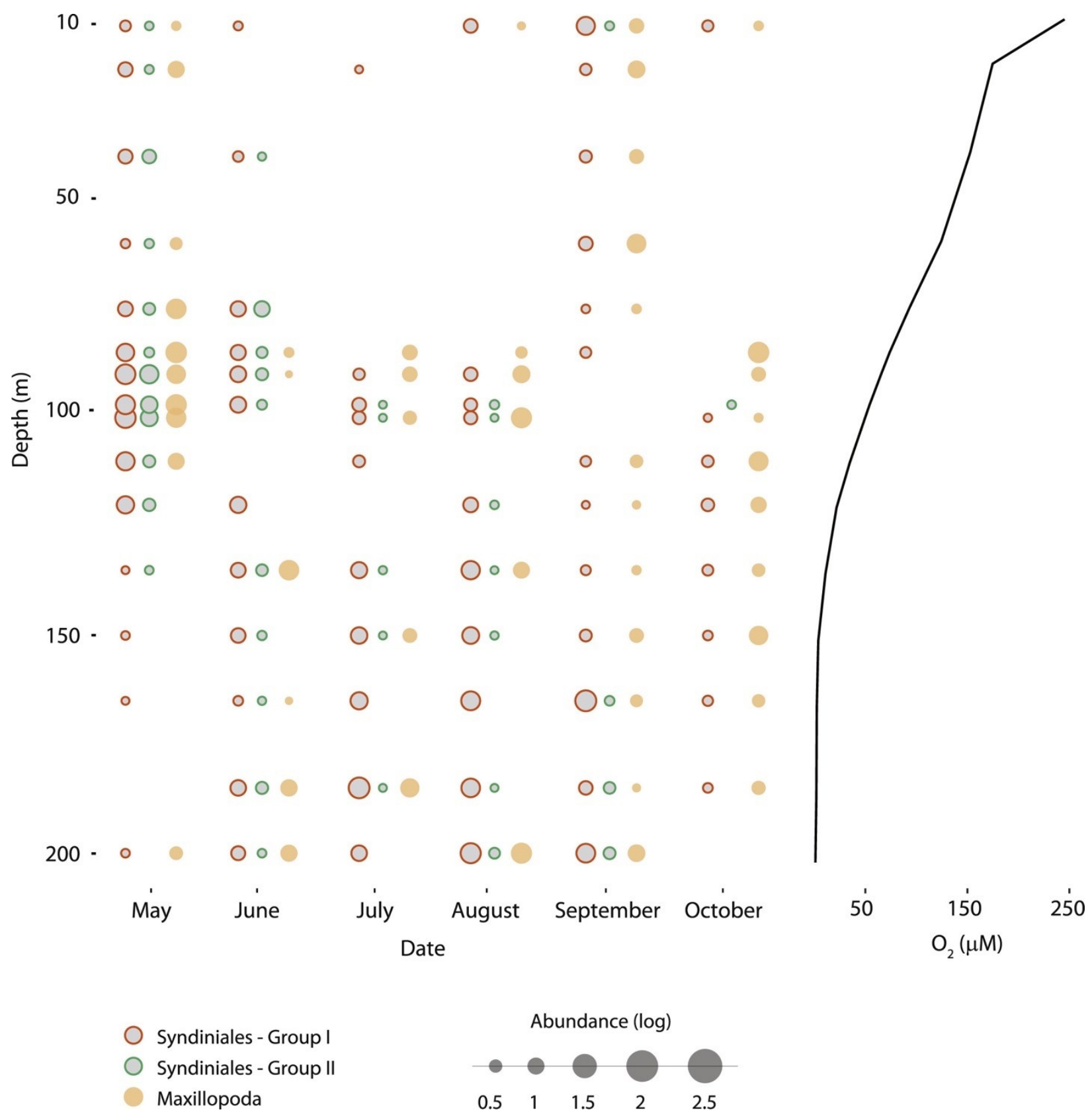

Figure 8. Vertical distribution and abundance of Maxillopoda and Syndiniales OTUs through the water column from May-October 2008. Dots size depicts OTUs relative abundance in logarithmic scale. 


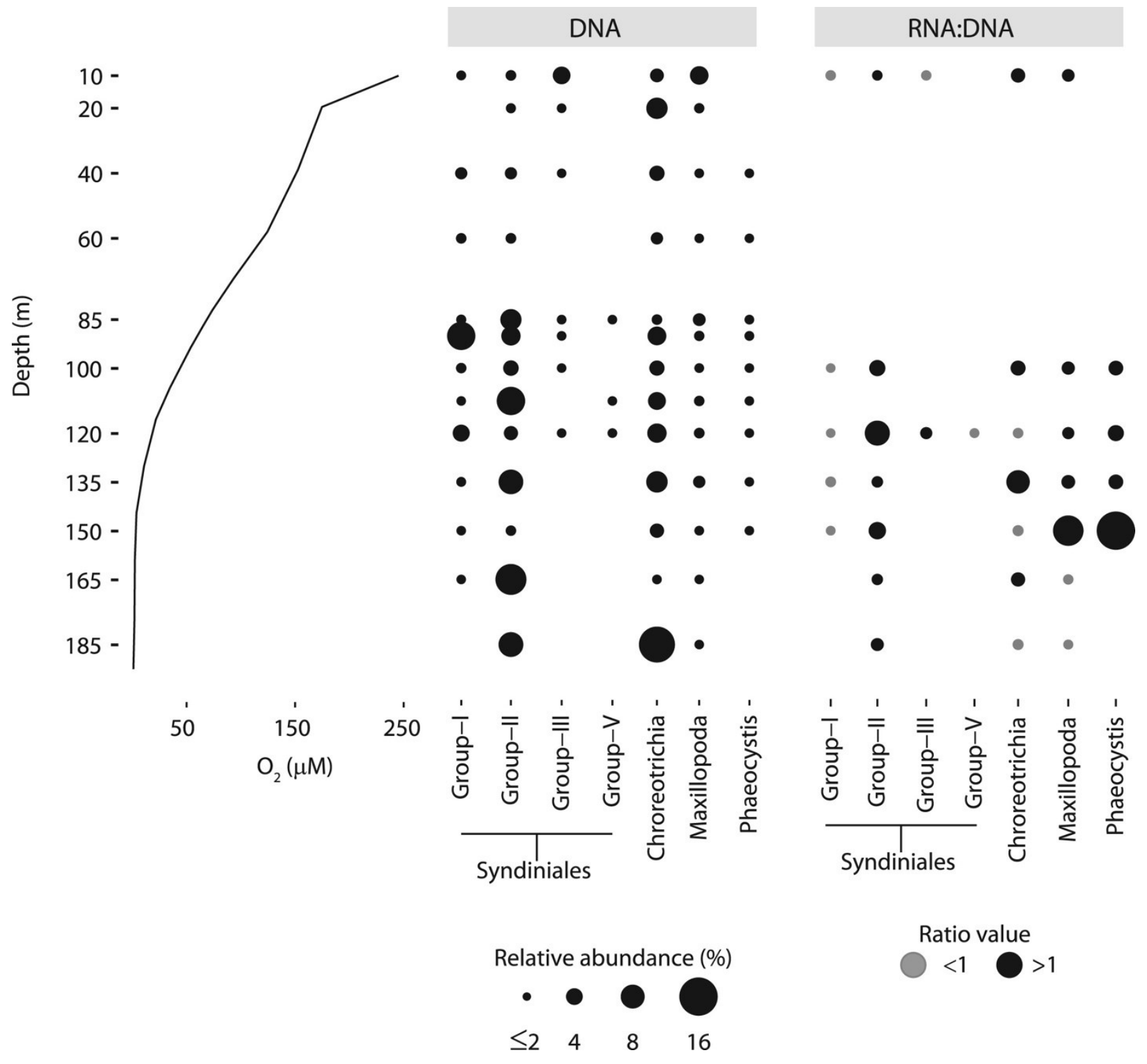

Figure 9. Distribution of Syndiniales and interacting Choreotrichia, Phaeocystis sp., and Maxillopoda OTUs throughout the water column oxygen gradient during the stratification period of July 2014. Dissolved oxygen concentration $(\mu \mathrm{M})$ profile is shown as a black sparkline on the left figure panel. OTUs vertical distribution is shown as dots which size depicts the relative abundance (\%) of the OTUs affiliated to each taxonomic group calculated out of the total number of reads in the SSU rDNA pyrotag dataset. The rRNA:rDNA ratio throughout the water column for the OTUs affiliated to each taxonomic group is shown as size and color constrained dots $($ gray $<1$; black $>1)$. 


\section{SUPPLEMENTARY INFORMATION}

Protistan parasites along oxygen gradients in a seasonally anoxic fjord: a network approach to assessing potential host-parasite interactions

Mónica Torres-Beltrán ${ }^{1 *}$, Taylor Sehein ${ }^{2 *}$, Maria Pachiadaki ${ }^{2,3}$, Steven Hallam ${ }^{1,4,5,6}$, Virginia $\mathrm{Edgcomb}^{2 \wedge}$

*Authors contributed equally to this work

$\wedge^{\wedge}$ Correspondence: Virginia Edgcomb, vedgcomb@whoi.edu

\section{SUPPLEMENTARY RESULTS}

Co-occurrence analysis and network

To determine potential interactions between OTUs throughout the water column over the stratification period (May-August) in Saanich Inlet, a co-occurrence network was constructed using both Bray-Curtis and Spearman correlation measures. All statistically significant correlations among OTUs resulting after permutations and bootstrap score distributions were included in the downstream analyses. The final edges matrix was visualized as a force directed network using Cytoscape 2.8.3 (Shannon et al., 2003). Each node represents an OTU and each edge a statistically significant positive correlation indicating co-occurrence. The resulting network contains 325 nodes, connected by 6,273 edges. Average node degree (mean edges per node) (Proulx et al., 2005) was 3, the average path length (the expected distance between two connected nodes) (Latora and Marchiori, 2009) was 2.73, and the network diameter (longest path between two nodes) (Cardoso et al., 2009) was equal to 8 . The clustering coefficient (connectedness of a node's neighbour) (Proulx et al., 2005) was 0.518, and connectance (proportion of all possible links realized) (Dunne et al., 2002) was 0.05 .

To describe the potential interactions occurring among Syndiniales OTUs and protists, OTUs were taxonomically identified in the Cytoscape network. Nodes corresponding to Syndiniales and their pairs were highlighted to summarize interactions (Supplementary Table 5). Based on nodes OTU number correlation data was extracted and exported for co-occurrence description and visualization. 


\section{SUPPLEMENTARY FIGURE}
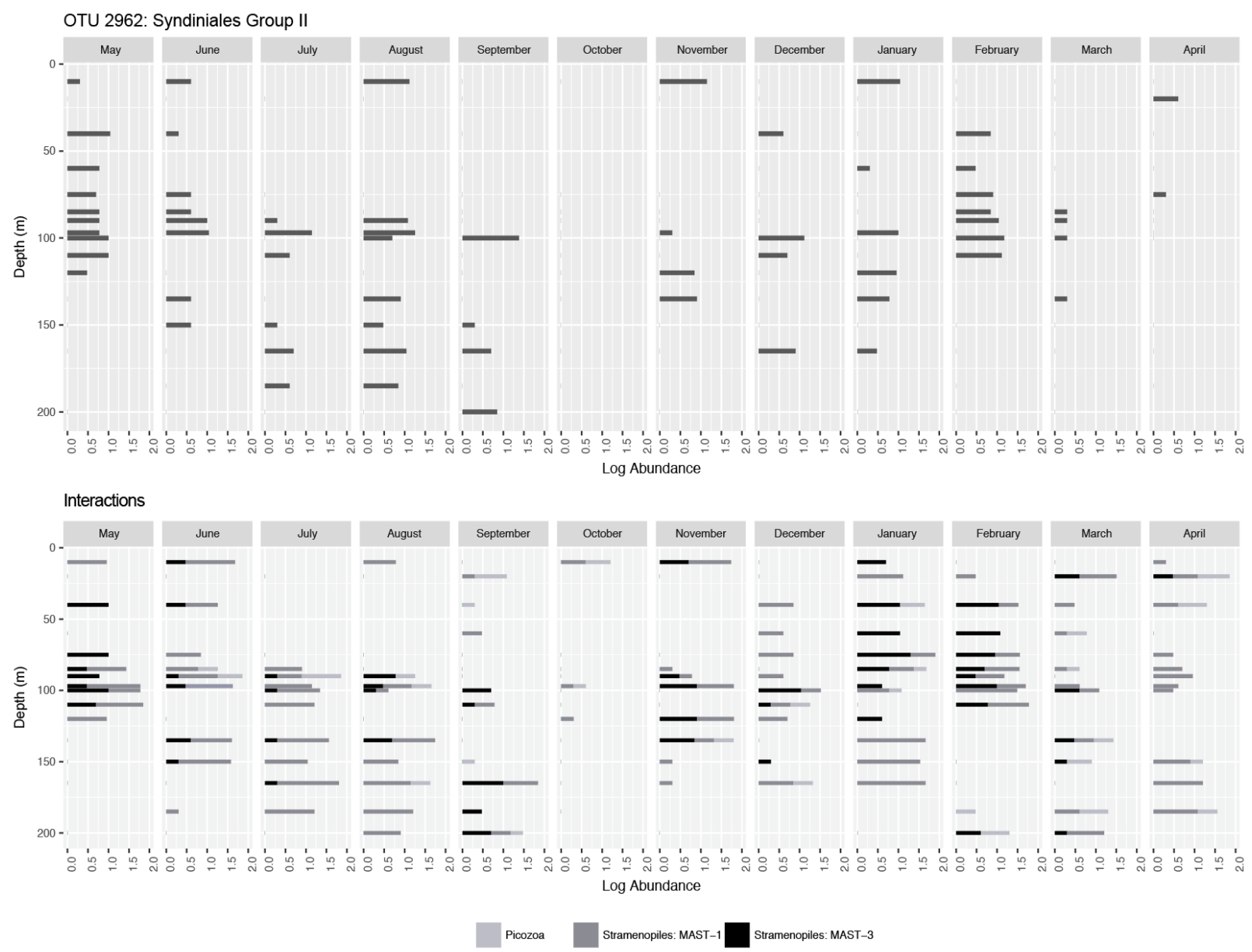

Supplementary Figure 1. Vertical distribution overtime for Syndiniales, Picobiliphyta, and Stramenopiles MAST1 and 3 OTUs. Top: Bars depict the abundance of Syndiniales OTUs found to correlate with Picobiliphyta, and Stramenopiles MAST1 and 3 throughout the water column from May 2008 to April 2009. Bottom: Stacked bars depict the abundance (log transformed) for the taxa OTUs found to correlate with Syndiniales throughout the water column from May 2008 to April 2009. 


\section{SUPPLEMENTARY TABLES}

Supplementary Table 1. Indicator OTUs for summer stratification suboxic-dysoxic water column conditions from May to August 2008. The OTU Id number, and significant ( $\mathrm{p}=0.05, \alpha \leq 0.01$ ) indicator value are shown. Selected OTUs potentially showing a parasite-type interaction are highlighted.

Supplementary Table 2. List of Spring indicator OTUs (May-June 2008). Table shows the OTU Id number, indicator value (IV $\geq 0.6$, $p$-value $\leq 0.05$ and $\alpha$ value $\leq 0.01$ ), and BLAST-based taxonomic assignment.

Supplementary Table 3. List of Summer stratification indicator OTUs (July- August 2008). Table shows the OTU Id number, indicator value (IV $\geq 0.6$, $p$-value $\leq 0.05$ and $\alpha$ value $\leq 0.01$ ), and BLAST-based taxonomic assignment.

Supplementary Table 4. List of early Fall renewal indicator OTUs (September-October 2008). Table shows the OTU Id number, indicator value (IV $\geq 0.6$, $p$-value $\leq 0.05$ and $\alpha$ value $\leq 0.01$ ), and BLAST-based taxonomic assignment.

Supplementary Table 5. Complete list of interaction found over the stratification water column conditions from May to August 2008. The OTU Id number for Paired OTUs (source and pair) with their corresponding taxonomic assignment is shown. All interactions listed were found significant $(p=0.05, \alpha \leq 0.01)$ with correlation values higher than 0.6 for Bray-Curtis and Spearman correlations.

*Supplementary material available online 


\section{REFERENCES}

Cardoso, J.C.S., Baquero, C., and Almeida, P.S., 2009. Probabilistic Estimation of Network Size and Diameter, Dependable Computing, 2009. LADC '09. Fourth Latin-American Symposium on, pp.33-40.

Dunne, J.A., Williams, R.J., and Martinez, N.D., 2002. Food-web structure and network theory: The role of connectance and size. Proceedings of the National Academy of Sciences, 99, pp.12917-12922.

Latora, V. and Marchiori, M., 2002. Is the Boston subway a small-world network? Physica A: Statistical Mechanics and its Applications, 314(1-4), pp.109-113.

Proulx, S.R., Promislow, D.E.L., and Phillips, P.C., 2005. Network thinking in ecology and evolution. Trends in Ecology and Evolution, 20, pp.345-353.

Shannon, P., Markiel, A., Ozier, O., Baliga, N.S., Wang, J.T., Ramage, D., Amin, N., Schwikowski, B., and Ideker, T., 2003. Cytoscape: A Software Environment for Integrated Models of Biomolecular Interaction Networks. Genome Research, 13, pp.2498-2504. 


\section{CHAPTER TWO}

Parasitic infections by Group II Syndiniales target select dinoflagellate host populations within diverse protist assemblages in a model coastal pond

Taylor R. Sehein ${ }^{1}$, Rebecca J. Gast ${ }^{2}$, Maria Pachiadaki ${ }^{2}$, Laure Guillou ${ }^{3}$, Virginia P. Edgcomb ${ }^{4}$

${ }^{1}$ MIT-WHOI Joint Program in Biological Oceanography, Cambridge and Woods Hole, MA, United States

${ }^{2}$ Department of Biology, Woods Hole Oceanographic Institution, Woods Hole, MA, United States

${ }^{3}$ Sorbonne Université \& Centre National pour la Recherche Scientifique, Station Biologique de Roscoff, Roscoff, France

${ }^{4}$ Department of Geology and Geophysics, Woods Hole Oceanographic Institution, Woods Hole, MA, United States

Corresponding Author:

Taylor Sehein

266 Woods Hole Road, MS\#8, Woods Hole, MA 02543

(508) 289-3064

tsehein@whoi.edu

\section{STATEMENT OF CONTRIBUTION}

Samples were collected and processed by Taylor Sehein with assistance from Virginia Edgcomb, Rebecca Gast, Maria Pachiadaki, and other members of the Edgcomb lab. Sequence data was processed and analyzed by TS with input from Laure, Guillou, RG, MP, and VE. Microscopy work and cell counts were performed by TS. TS wrote the manuscript and created the figures with input from the co-authors. 


\title{
SIGNIFICANCE STATEMENT
}

High-resolution time series data in a shallow, stratified, coastal saline pond revealed a seasonal diversity of syndinian parasite and host morphotypes, with both specific and generalist parasitic infections. These events occurred in mixed protist communities, and the absence of blooms dominated by a single species provided a new perspective on the activity of syndinian parasites in marine environments.

\begin{abstract}
Protists are integral to marine food webs and biogeochemical cycles; however, there is a paucity of data describing specific ecological niches for some of the most abundant taxa in marker gene libraries. Syndiniales are one such group, often representing the majority of sequence reads recovered from picoplankton samples. Across the global ocean, however, the prevalence and impacts of syndinian parasitism in marine environments remains unclear. We began to address these critical knowledge gaps by generating a high-resolution time series (March-October 2018) in a productive coastal pond. Seasonal shifts in protist populations, including parasitic Syndiniales, were documented during periods of higher primary productivity and increased summer temperature-driven stratification. Elevated concentrations of infected hosts and freeliving parasite spores occurred at nearly monthly intervals in July, August, and September. We suggest intensifying stratification during this period correlated with the increased prevalence of dinoflagellates that were parasitized by Group II Syndiniales. Infections in some protist populations were comparable to previously reported large single-taxon dinoflagellate blooms. Infection dynamics in Salt Pond demonstrated the propagation of syndinian parasites through mixed protist assemblages and highlighted patterns of host/parasite interactions that better reflect many other marine environments where single taxon blooms are uncommon.
\end{abstract}

\section{INTRODUCTION}

Coastal oceans are highly productive systems that support complex marine food webs and play important roles in the cycling of carbon and other nutrients. Within these environments, protists are taxonomically diverse drivers of energy and nutrient flow through their activities as primary producers or as parasites, heterotrophs or mixotrophs that facilitate the trophic transfer of energy between prokaryotes (Bacteria, Archaea) and Metazoa (Aristegui et al., 2009) and can exert topdown controls on certain populations. Protists occupy varied ecological niches; however, the 
common trophic strategies of mixotrophy and symbioses (parasitism and symbiotic mutualistic associations), are often poorly understood and unrecognized in models of marine food webs (Jephcott et al., 2016, Scholz et al., 2016, Frenken et al., 2017, Stoecker et al., 2017). Elucidating the extent and consequences of specific protistan interactions is critical for understanding microbial community structure and dynamics, as well as overall ecosystem function in the global ocean.

Molecular marker surveys of the eukaryotic small subunit ribosomal RNA (SSU rRNA) gene over the past two decades indicate protist parasites are abundant and likely to play significant roles in diverse marine ecosystems. For example, the Tara Oceans project surveyed the tropical sunlit and mesopelagic ocean and showed that parasitic protist groups accounted for up to $59 \%$ of total heterotrophic protist SSU rRNA gene richness and approximately $53 \%$ of abundance. Among these parasitic sequences, $89 \%$ of picoplankton diversity and abundance were affiliated with Syndiniales Groups I and II (phylum Dinoflagellata) (Pawlowski et al., 2012, de Vargas et al., 2015). The Malaspina-2010 Expedition found that four protist groups accounted for $69.6 \%$ of sequencing tags in the bathypelagic global ocean, and Group II Syndiniales sequences were the most highly represented, and phylogenetically diverse group in most Malaspina samples (Pernice et al., 2015). Similar results were obtained in other environmental surveys of polar regions and oxygen-minimum zones (Cleary and Durbin, 2016, Orsi et al., 2012, Duret et al., 2015). Collectively, surveys of SSU rRNA gene signatures suggest Syndiniales are abundant and ubiquitous in both coastal and open ocean environments, and likely play a major role in those marine food webs (e.g. Lopez-Garcia et al., 2001, Massana et al., 2004, Not et al., 2007, Edgcomb et al., 2011, Pernice et al., 2015, de Vargas et al., 2015). Very little is known about the enigmatic Syndiniales lineages, beside the fact that all species formally described to date have a parasitic lifestyle (Cachon 1964, Guillou et al., 2008). A few of them are known to be an important cause of mortality for microbial eukaryotes and Metazoa (Skovgaard et al., 2005, Stentiford and Shields, 2005, Chambouvet et al., 2008).

Fundamental challenges when studying syndinian parasites and putative host interactions are their unresolved taxonomy and the paucity of morphological markers for different clades within this order. Syndiniales includes five distinct taxonomic groups (Guillou et al., 2008). Sequences from environmental datasets are most commonly assigned to Syndiniales Groups I and II, which include eight and >100 clades, respectively (Guillou et al., 2008, Cai et al 2020). 
Group I, which includes the syndinian genus Eudoboscquella, is associated with infections of ciliates (Bachvaroff et al., 2012). The Amoebophrya species complex is the only described Group II Syndiniales genus, and this taxon is known to infect other dinoflagellates (Cai et al., 2020, Guillou et al., 2008, Chambouvet et al., 2008). Recent expansion of environmental sequence data in public databases suggests the Amoebophrya species complex is affiliated with multiple clades within Group II, indicating that the group's taxonomy should be revisited, as well as the definition of the genus (Guillou et al., 2008, Cai et al. 2020). Other known hosts of syndinian parasites include radiolarians and copepods (Skovgaard, 2014, Brate et al., 2012); however, there are many clades within both Groups I and II that remain known only by their marker gene sequences and no information is available on host taxa.

Group II Syndiniales infection cycles have been described for a limited number of cultured isolates infecting dinoflagellates, with most belonging to the Amoebophrya species complex (Cachon, 1964, Miller et al., 2012, Cachon and Cachon, 1970). Infections lead to host mortality within several days, releasing nutrients to the environment both as particulate and dissolved organic matter; however, co-dormant infections within the resting stage of the dinoflagellate host, Scrippsiella acuminata, have been observed in culture (Chambouvet et al., 2011). Infections generate dozens to hundreds of new propagules (dinospores) that are between 1 and $12 \mu \mathrm{m}$ in diameter. Culture-based studies suggest dinospores survive only a few days after their release (Coats and Park, 2002). Assuming the life cycles of other uncultured Group II Syndiniales are similarly short, the dominance of their sequences in molecular datasets suggests their production in marine ecosystems is fairly constant.

An outstanding ecological question regards the impact of syndinian infections on protist populations and on the overall protist community. Survival of syndinian parasites, however, appears dependent on the availability of susceptible hosts. Previous studies of syndinian ecology have focused on a limited number of hosts through microscopic observations; however, the small size and lack of defining morphological features make dinospores and early stage infections difficult to identify and enumerate. Catalyzed reporter deposition fluorescence in-situ hybridization (CARD-FISH) is a more sensitive approach than light microscopy and has been used to successfully quantify free-living dinospores and infections in coastal, open ocean, and oligotrophic ocean water masses (Chambouvet et al., 2011b, Siano et al., 2011, Velo-Suarez et al., 2013). To date, several CARD-FISH probes have been designed to detect syndinian parasites, 
and one probe targets 73 of the 125 clades of Group II Syndiniales (ALV01, Chambouvet et al., 2008). Applications of CARD-FISH show that Group II parasite infections increase in abundance during the peak through the termination of some dinoflagellate blooms, including some harmful algal bloom taxa (Coats and Bockstahler, 1994, Coats and Park, 2002, VeloSuarez et al., 2013). This suggests syndinian parasites may exert top-down pressure on bloomforming taxa. In the Penzé Estuary (Roscoff, France), up to $46 \%$ of dinoflagellates were infected (average of 21\%) over a 3-month period during the summer (Chambouvet et al., 2008). Infection ratios were similar among the five dinoflagellate species that could be distinguished based on morphology, including the toxic alga Alexandrium minutum. Based on observed characteristic predator-prey dynamics in coastal settings, proliferation of parasite infections should occur when there is a high concentration of preferred hosts (Velo-Suarez et al., 2013).

CARD-FISH probes and epifluorescent microscopy can highlight interactions between hosts with distinct morphologies (Siano et al., 2011) and Group II Syndiniales parasites for which clade-specific probes have been designed (Chambouvet et al., 2008). Co-occurrence network analyses can be used to infer putative host-parasite interactions based on the copresence of host and parasite taxa. Recent research has hypothesized syndinian parasites may infect diatoms (Sassenhagen et al., 2020) as well as the marine haptophyte, Phaeocystis (Christaki et al., 2017, Torres-Beltran et al., 2018). These findings indicate the host range for Group II Syndiniales could be broader than previously thought and encourages exploration of a broader range of marine systems.

Salt Pond (Falmouth, MA, USA) was selected for a high-resolution time series study of syndinian infection dynamics because it is a good model for a typical, productive coastal site in the temperate Atlantic. Unlike previous studies of Group II Syndiniales that targeted regions where blooms of dinoflagellate hosts were prominent, little was known about the protist communities in Salt Pond and the impacts of Group II Syndiniales on individual protist populations. We combined molecular marker gene analyses, fluorescence in-situ hybridization microscopy, and data on water chemistry to water samples collected every three days from March-October 2018. This timeframe was selected to capture periods of increased putative host cell densities, including anticipated spring and fall blooms of primary producers that are typical of the region, as well as mid-summer, temperature-driven stratification that concentrates protist cells across the oxic/anoxic interface (Bazylinski et al., 2000). We hypothesized that in Salt 
Pond, Group II Syndiniales infections would propagate more efficiently when there were higher concentrations of host populations.

\section{METHODS}

Study site

Salt Pond (Falmouth, MA; $41.5430^{\circ} \mathrm{N}, 70.6269^{\circ} \mathrm{W}$, Supplementary Figure S1) is within two miles of Woods Hole Oceanographic Institution and easily accessible by small row boat, thus facilitating the sampling frequency needed for a high-resolution time series that can capture most parasite infection dynamics. This estuarine system is approximately $5.5 \mathrm{~m}$ deep at the sampling site for this study, tidally influenced by the Vineyard Sound, and receives freshwater inputs primarily from groundwater. Salt Pond is seasonally stratified, with oxygen depleted waters that occur within 2-3 meters of the water surface in late summer, and bottom waters that become increasingly sulfidic as stratification intensifies in late summer (reaching up to $\sim 5 \mathrm{mM}$; Wakeham et al., 1984, 1987, Zemmelink et al., 2006).

\section{Sample collection}

Water samples were collected approximately every three days between March and October 2018, which captured periods of increased primary productivity (spring and fall blooms) and seasonal stratification (summer). The pond was sampled at three depths at every time point. These depths correspond to the depths of oxic $(2 \mathrm{~m})$, oxycline $(3 \mathrm{~m})$, and anoxic $(4 \mathrm{~m})$ water conditions that occur during peak stratification. Temperature, salinity, and dissolved oxygen concentrations were profiled using a handheld YSI (YSI Pro2030, Ohio, USA). Two replicate 1.7L GoFlo (General Oceanics, Florida, USA) casts were performed at each depth. From each cast, 600mL of seawater was preserved using a final concentration of $4 \%$ unbuffered formalin. 1L of seawater from each cast was kept on ice and returned to the laboratory within one hour of collection.

In the laboratory, samples from each $1 \mathrm{~L}$ sample were processed and stored for amplicon and nitrogen analyses. For each cast, a $20 \mathrm{~mL}$ subsample of seawater was filtered onto a $47 \mathrm{~mm}$ $0.2 \mu \mathrm{m}$ Millipore Isopore filter that was placed directly in a DNeasy PowerWater Kit (Qiagen, USA) bead tube for DNA extraction. Bead tubes were stored at $-20^{\circ} \mathrm{C}$ until extractions were performed. Seawater was processed for water chemistry analyses by filtering through a $0.2 \mu \mathrm{m}$ Sterivex filter, and collecting 50mL in a HDPE Nalgene bottle for measuring ammonium, nitrate and nitrite concentrations. These HDPE bottles were stored at $-20^{\circ} \mathrm{C}$ until analyzed on an O.I. 
Analytical Flow Solutions IV auto analyzer at the Louisiana State University Wetland Biogeochemistry Analytical Services facility. Corresponding formalin-fixed seawater samples were subsampled to visualize both free-living parasites and infected hosts. $20 \mathrm{~mL}$ of formalinfixed seawater was filtered onto a $47 \mathrm{~mm} 0.2 \mu \mathrm{m}$ Millipore Isopore filter (black) for enumeration of free-living parasite spores. Filters were rinsed three times with $5 \mathrm{~mL}$ phosphate buffered saline (PBS, $1 \mathrm{x}$ ) and stored at $-20^{\circ} \mathrm{C}$. To enumerate infected hosts, $100 \mathrm{~mL}$ or $200 \mathrm{~mL}$ (determined by filtering time) of fixed seawater was filtered onto a $47 \mathrm{~mm} 3.0 \mu \mathrm{m}$ Millipore Isopore filter, rinsed three times with PBS and stored at $-20^{\circ} \mathrm{C}$.

Catalyzed reporter deposition fluorescence in-situ hybridization (CARD-FISH) Filters for enumerating infected hosts and numbers of dinospores were hybridized to a horseradish peroxidase oligonucleotide probe targeting 73 of the 125 clades of Group II Syndiniales (Cai et al., 2020; ALV01). Filters were cut into 8 wedges and one wedge per sample was placed on a glass slide. $20 \mu \mathrm{L}$ of hybridization buffer containing $40 \%$ formamide (Chambouvet et al. 2008) and the probe ALV01 (5'- GCC TGC CGT GAA CAC TCT -3'; Chambouvet et al. 2008) in a 9:1 ratio (final concentration 50ng $\mu \mathrm{L}^{-1}$ ) was placed on each filter piece and spread using the side of a pipette tip. Slides were placed into $50 \mathrm{~mL}$ Falcon tubes on their side with a Kimwipe placed at the bottom of the chamber saturated with $1 \mathrm{~mL}$ of hybridization buffer. Samples were incubated for a minimum of 8 hours at $37^{\circ} \mathrm{C}$. Following the initial hybridization, filter pieces were washed in $3 \mathrm{ml}$ of a freshly-prepared wash solution twice at $46^{\circ} \mathrm{C}$ to remove unhybridized probe, then soaked in $3 \mathrm{~mL}$ of Tris-HCl-NaCl-Tween $20(1 \mathrm{M}$ Tris- $\mathrm{HCl} \mathrm{pH} 7.5$, Tween, $30 \mathrm{ml} / \mathrm{L} 5 \mathrm{M} \mathrm{NaCl}$; TNT) buffer at room temperature and placed on a second glass slide. Filters were transferred to new glass slides and fluorescence was amplified using the Perkin Elmer tyramide signal amplification kit (Lot: NEL741001KT) according to the manufacturer's instructions, and filters were then washed twice in $3 \mathrm{ml}$ of TNT buffer at $55^{\circ} \mathrm{C}$ to neutralize any residual fluorescein. Filters were placed on a glass slide and Calcofluor White (Sigma Aldrich; final concentration 2.5\% [vol/vol]) was used to stain cellulose within the cell wall of thecate dinoflagellates. Filters were stained for 7 minutes and then washed in $3 \mathrm{ml}$ of water twice for 1 minute. Filters were counterstained with the nucleic acid stain propidium iodide and mounted on glass slides with Citifluor mounting media $(10 \mu \mathrm{g}$ per mL Citifluor; $20 \mu \mathrm{L}$ 
mounting solution per slide). Hybridized filters were stored at $4{ }^{\circ} \mathrm{C}$. A more detailed description of the protocol can be found at dx.doi.org/10.17504/protocols.io.bsxmnfk6.

Enumeration of free-living dinospores and infected hosts

All microscope analyses were performed on an Axio Imager M2 epifluorescence microscope (Carl Zeiss, Germany). Hybridized filters were viewed using the FITC filter set, which excites the fluorescein stain coupled to the CARD-FISH probe targeting both free-living parasites and infected cells. The DAPI filter set was used to visualize the presence or absence of host thecae stained with Calcofluor White, and the Cy3 filter set was used to detect the propidium iodide stain and confirm the presence of a nucleus in all putative parasite cells. Free-living parasite spores, hybridized with the ALV01 probe, were enumerated under 400x magnification by counting all spores on 1/8-filter wedges. To calculate overall protist community infection levels within the fraction of the eukaryotic community $10 \mu \mathrm{m}$ or larger), cells on filters were also hybridized with the ALV01 probe. All infected protist cells showing one or more infective spores within the cell were counted at 400x magnification. All eukaryotic cells within the ocular grid observed at 1000x were counted until either 400 protists or 40 grids were reached. A "cells per grid" value was calculated and multiplied by the total number of grids on $1 / 8$ of the filter, which was calibrated by comparing the ocular grid to a stage micrometer slide, to estimate total protist abundance. Values for numbers of infected hosts are expressed as the average percent of the total protist community infected based on counts performed for the two replicate casts at each depth.

DNA extraction and iTag sequencing DNA was extracted with the Qiagen DNeasy PowerWater kit following the manufacturer's protocols, eluted in a final volume of $50 \mu \mathrm{L}$, quantified using a Qubit Fluorometer, and stored at $20^{\circ}$ C. 339 samples with sufficient DNA extracted were selected for sequencing and shipped to Georgia Genomics and Bioinformatics Core (University of Georgia) for iTag barcoding and sequencing on the Illumina MiSeq platform using primers for the V4 hypervariable region of the eukaryotic 18S rRNA gene, V4F (5'-CCA GCA SCY GCG GTA ATT CC-3'; Stoeck et al. 2010) and V4RB (5'-ACT TTC GTT CTT GAT YRR-3'; Balzano et al. 2015). The QIIME2 platform and the dada2 pipeline were used to process the sequence data (Bolyen et al., 2019). Reads were quality checked and chimeras were removed. Amplicon sequence variants (ASVs) 
were clustered based on $100 \%$ similarity and representative sequences were aligned and annotated using the PR2 (Guillou et al. 2013) database following standard procedures (Bolyen et al. 2019). All sequences were submitted to GenBank and made publicly available (BioProject ID PRJNA736596).

\section{Statistical analyses}

All statistical analyses were performed in RStudio (Version 1.1.456) using R (Version 3.6.1).

ITag barcode libraries were manually curated, removing amplicon sequence variants (ASVs) that could not be identified to phylum and those annotated to Metazoa and macroalgae. Libraries from replicate casts collected at the same depth on each sampling date were combined.

To evaluate changes in community composition across the time series, non-metric multidimensional scaling was performed using the MASS package (v7.3-51.6; Venables and Ripley, 2002) in R. ASV read counts were transformed using the Hellinger method using decostand in the vegan package (v2.4-2; Oksanen et al., 2017). A dissimilarity index was then constructed using the Bray-Curtis method using vegdist. Points were fitted using isoMDS in the MASS package, which is based on the Kruskal method. Environmental parameters, including temperature, salinity, dissolved oxygen, ammonium, nitrate and nitrite, moon phase, day length, average wind speed, maximum gust, and wind direction were fitted to the points on the ordination plot using the envfit function in the vegan package with 999 permutations. Abiotic factors with p-values less than 0.05 are presented. A correlation matrix of environmental parameters was constructed using the rcorr function in the Hmisc package (v4.6-0; Harrell, 2021) in R. Significantly correlated parameters $(\mathrm{p}<0.05)$ are summarized in Figure S2.

The ASV read count table was then used to determine clusters of co-occurring protist taxa and the temporal dynamics of these community clusters using methods outlined in Coenen et al. (2019). Read counts were normalized to scale and detrend the data. Variances were stabilized so that they no longer correlated with ASV abundances using the varianceStabilizingTransformation function in the DeSeq2 package (v1.39.0; Love et al., 2014). Linear trends were removed using the detrend function in the pracama package (v2.3.3; Borchers, H.W.) and count values were rescaled. The normalized data, or Z-scores, were used to generate a Euclidean distance matrix with the dist function in the stats package (v3.6.2; R Core Team), and to subsequently define protistan community clusters. Clusters were defined using the 
k-medoids method with the pam function in the cluster package (v2.1.2; Maechler et al., 2021). All scripts used for this analysis can be found at https://github.com/trsehein/SaltPond_iTagAnalysis.

\section{RESULTS}

Seasonality and environmental conditions in Salt Pond

Temperature and salinity increased from spring into summer at the shallowest depth we sampled in Salt Pond ( $2 \mathrm{~m}$ below the surface), with salinity values at $3 \mathrm{~m}$ and $4 \mathrm{~m}$ more uniform across the time series (Figure 1; Table S1). Oxygen concentrations declined with depth beginning in May, with undetectable values shoaling to approximately two meters on August 16 and these conditions persisting until September 4. Ammonium was depleted from all three depths beginning on May 14, with concentrations remaining low until July 30. Ammonium then began to accumulate in low-oxygen and anoxic samples collected at four meters. Concentrations of nitrate and nitrite were highest in spring months and were depleted at all sample depths in the summer and fall (Figure 1, Table S1).

\section{Spatial and temporal assessments of protist community composition}

Barcoded iTag libraries targeted the hypervariable V4 region of the 18S SSU rRNA gene. Across the time series, libraries for 339 samples were sequenced, generating 9,317,051 reads that were catalogued into 9,196 amplicon sequence variants (ASVs; Table S2) based on 100\% sequence similarity. ASVs were manually curated to remove sequences annotated only to Eukaryota, Metazoa, and red and green macroalgae, leaving 6,570 ASVs for protistan community analyses. Trends in community composition revealed seasonal shifts in the relative abundances of major groups including Cercozoa, chlorophytes, ciliates, dinoflagellates, and stramenopiles (Figure 2b). The relative abundances of these groups were similar across the three depths at each sampling event, with several exceptions. Chlorophytes were more abundant at the two-meter depth in early spring, ciliates were most abundant at four meters across most of the time series, and cryptophytes were most abundant at four meters in late July and early August.

The relative abundances of Group II Syndiniales parasite sequences (Figure 2a) exhibited periodicity across the times series. Local peaks in Group II Syndiniales sequences coincided with occurrence of dinoflagellate-dominated protist communities and with elevated infection events recorded using CARD-FISH and epifluorescence microscopy in July, August, and September. 
Group I Syndiniales sequences, commonly associated with ciliate infections, were most abundant in sample data sets at three and four meters in September and October. Sequences annotated to Group III Syndiniales, whose parasitic function and hosts are unknown, were nearly undetectable at two meters throughout the time series, but their sequences were abundant at three and four meters from mid-May to mid-June.

Non-metric multidimensional scaling analysis of the iTag libraries indicated protist communities grouped by sampling month (Figure 3). Abiotic factors related to seasonal changes, including temperature, salinity, dissolved oxygen, wind direction, maximum wind gust, and combined nitrate and nitrite concentrations, were all significantly associated with sample clustering. We calculated positive correlations between dissolved oxygen and nitrate+nitrite, day length, and wind direction, which likely covary due to seasonal patterns at temperate latitudes. Strong negative correlations were identified between temperature and dissolved oxygen and nitrate+nitrite, corresponding to changes in the water column associated with thermohaline stratification (Figure S2). Depth did not significantly influence the grouping of samples across the ordination plot.

Parasite enumeration: free-living spores and infected host morphologies The seasonal impacts of Syndiniales parasites on protist populations were evaluated by using CARD-FISH and fluorescence microscopy to enumerate both infected hosts and free-living dinospores. For infected host counts, 283 filters spanning the March-October 2018 sampling dates were hybridized from the three depths sampled (Figure 4). Filters from April 27 to May 11 were not inspected because no Syndiniales amplicons were present in the corresponding iTag samples. In-silico analysis of the ALV01 probe sequence revealed the probe matched 341 of 438 Group II ASVs from Salt Pond, representing 93.4\% of Group II Syndiniales reads. Infections were detected in a small fraction of the overall protist community throughout most of the time series, with three infection events occurring significantly above background levels at nearly monthly intervals in July, August, and September. The highest prevalence of infected protists, 2.56\%, was observed on August 13 at 3 meters depth. Additional peaks in infections occurred on July 3 and September 14 at the 2 and 3-meter depths, illustrating periodicity in infection cycles across the summer months of 2018. Host morphologies were diverse and represented a range of athecate and thecate dinoflagellates, including heterocapsids, scrippsielloids, and dominant large 
athecate cells based on microscopic observations of characteristic morphologies (Figure S3). Infection frequencies were calculated for the three morphotypes (Figure S4). No infections of other dominant protist groups were evident, including ciliates and diatoms.

Because most infections occurred at the two meters depth, a subset of filters around the three elevated infection periods was also selected to quantify free-living spore concentrations. Dinospore concentrations were enumerated in 34 samples and were found to reach similar local maxima concurrent with host infections on July 3 (335,000 spores/L) and September 14 (139,200 spores $/ \mathrm{L}$ ). No increase in spore concentration was observed for sampling dates on or around the August 13 infection peak (Figure 4).

\section{Clustering analyses and protist community succession}

Additional statistical analyses examined the relative abundance of protist taxa in the iTag libraries for samples collected from two meters depth where most infections were observed (Figure 4). "Universal" primers have the potential to miss some fraction of the in-situ community, and in this case, it appeared to miss a dominant euglenoid morphotype that microscopy revealed formed blooms of millions of cells/L at multiple time points across the time series at the three- and four-meter depths. These euglenoids were not infected by Group II Syndiniales. Given the general similarities of protist communities across depths illustrated by the NMDS analysis, we focused attention on iTag libraries from the two-meter samples where observed infections were highest, to characterize cohorts of co-occurring protist populations.

The k-medoids method was used to parse the iTag libraries into patterns of co-occurring protist taxa. This analysis revealed four cohorts representing distinct protist communities that appeared at different times across the sampling period (Figure 5). The taxonomic diversity of each cluster is listed in Table S3. Clusters were identified across the time series by calculating zscores, which describe the combined presence (positive values) and absence (negative values) of all taxa within each cluster. Here, only the positive values are presented, indicating the taxa within a cluster were present above mean baseline values on a given sampling date. Cluster four was present in both early spring and early fall samples, which suggests these protist taxa exhibit temporal periodicity in the pond, perhaps influenced by temperature. Other clusters, including clusters one, two, and three, occurred once during the time series, and persisted for approximately one month each. 
Syndiniales diversity during infection events

470 ASVs were annotated to the order Syndiniales, representing 7.15\% of all protist taxonomic annotations across the time series. The abundance of Syndiniales reads within individual iTag barcode libraries ranged from zero on multiple dates to $19.39 \%$ on April 8 (Figure 2a). Like the overall protist community, the relative abundance of sequences from Syndiniales strains varied across the time series. In particular, Group II Syndiniales clade diversity differed during the three infection events observed in July, August, and September (Figure S5). Reads annotated to Group II Clade 2 represented approximately 97\% of all Group II Syndiniales on July 3. Likewise, over 94\% of Group II Syndiniales were annotated to Clade 30 on September 14. These mono-clade infection events provide key information about specific host-parasite interactions when putative

hosts can be identified morphologically or by using additional FISH analyses. On the other hand, the August 13 infection peak was uniquely diverse, with reads represented by six Group II Syndiniales clades $(1,2,4,5,14,22)$ and unidentified Group II sequences.

\section{DISCUSSION}

The coastal tidal pond, Salt Pond (Falmouth, MA, USA, Figure S1) is an example of a highlyproductive coastal ecosystem, with features resembling many other coastal ponds formed by glacial activity in the region. Salt Pond is a natural laboratory for studying parasitism by Syndiniales during the seasonal succession of protist populations, as well as the effects of stratification on parasitic protist interactions. Salt Pond has been studied extensively by researchers interested in magnetotactic bacteria (Simmons et al., 2004, Simmons et al., 2007, Moskowitz et al., 2008) and sulfur compounds that accumulate in the chemocline (Wakeham et al., 1984, Zimmelink et al., 2006); however, little was known about its protist community, including the diversity of Group II Syndiniales, or how stratification might influence protist distribution and syndinian interactions with hosts in the water column. Based on previous studies of syndinian impacts on bloom-forming dinoflagellate populations (Chambouvet et al., 2008, Siano et al., 2011, Velo-Suarez et al., 2013), we hypothesized infections would be more prevalent during seasonal bloom cycles and periods of stratification when most hosts were concentrated in the upper water column. Our time series sampling captured periods of increased primary production (spring and fall blooms) as well as stratification in the late summer. 
Intensifying stratification alters environmental variables in Salt Pond

Salt Pond is approximately 5.5 meters deep at its deepest point and the water column is structured by thermohaline stratification between mid-July and mid-September. Tidal mixing of freshwater from groundwater inputs and saltwater from the neighboring Vineyard Sound results in waters that are more saline with depth, and often cooler (Figure 1). Differences in the temperatures recorded at two-meters and four-meters increased from mid-June to midSeptember, with intensifying stratification of the water column.

Oxygen concentrations in the pond varied across the time series. Oxygen increased with depth in the early spring (March 19-May 7) suggesting elevated primary production and vertical mixing with oxygenated surface waters, but concentrations declined rapidly at four meters through May. Concentrations at three-meters declined in mid-June, concurrent with increased stratification of the water column. Concentrations of ammonium and both nitrate and nitrite were measured at the three sampling depths across the time series, and exhibited declines beginning in mid-May. Ammonium concentrations later increased at four meters depth between September and the end of the time series. These trends in oxygen and nitrogen concentrations during the intensification of thermohaline stratification in the summer months are consistent with publications on Salt Pond from decades ago (Wakeham et al., 1984, 1987), suggesting Salt Pond is a relatively stable system with changes in environmental parameters primarily driven by seasonal forcing.

Seasonal influences on the Salt Pond protist community

Salt Pond protist communities included diverse assemblages of dinoflagellates, diatoms, ciliates, chlorophytes, cryptophytes, and cercozoa (Figure $2 b$ ). While the relative abundance of taxonomic groups in iTag libraries can be biased by primer choice (Balzano et al., 2015), the number of gene copies per cell (Prokopowich et al., 2003, Gong and Marchetti, 2019), and amplification methods; examination of relative abundances of different protist taxa provides a view into general protist community trends. In Salt Pond, ASVs affiliated with dinoflagellates and diatoms comprised the two most abundant protist groups and exhibited niche differentiation across the time series. Shifts from diatom-dominated to dinoflagellate-dominated communities have been observed in many other marine settings (Trigueros and Orive, 2001, Zhou et al., 2017, Spilling et al., 2018); however, the timing of these shifts in association with biotic interactions (such as parasitism) is less understood. 
In temperate coastal settings, a period of elevated primary production termed the spring bloom occurs in response to warming waters, decreased vertical mixing, and replete nutrients (Sverdrup, 1953, Mallin et al., 1991, Yoshie et al., 2003, Silva et al., 2009, Jin et al., 2013, Carstensen et al., 2015). Spring protist communities described by the iTag libraries in Salt Pond were dominated by diatoms and chlorophytes (Figure 2), suggesting a bloom of these primary producers. The elevated oxygen concentrations throughout the water column recorded between March 19 and May 7 also indicate increased primary production. A second shift toward a diatom-dominated community occurred in the transition from late August to early September when peak stratification occurred (August 9-September 24) and sub-oxic conditions shoaled to approximately two meters. During this peak stratification ammonium, nitrate and nitrite were depleted in the upper water column $(0.0-2.89 \mathrm{mg} / \mathrm{L}$, average $1.23 \mathrm{mg} / \mathrm{L} ; 0.01-0.85 \mathrm{mg} / \mathrm{L}$, average $0.14 \mathrm{mg} / \mathrm{L}$ for ammonium and nitrate+nitrite, respectively). Diatoms abundant in August and September (Chaetoceros, Navicula, and Skeletonema) differed from species present during the spring bloom (Thalassiosira, Pinnularia, and Leptocylindrus), and may have adaptations to nutrient-limited conditions or the ability to scavenge nutrient hot-spots created by biotic interactions (i.e. parasitism) between other protists.

Many dinoflagellates are adapted to nutrient-limited environments due to their metabolic flexibility (mixotrophy in many species). iTag libraries from late June until mid-August and between September and the end of October indicated protist communities were dominated by dinoflagellates at all depths sampled. Dinoflagellates are known to have high numbers of SSU rRNA gene copies (Not et al., 2009, Galluzzi et al., 2010, Lin, 2011, Pernice et al., 2013), and this may lead to overestimation of their relative abundance; therefore, we enumerated the dominant morphotypes (heterocapsid, scrippsielloid, and large athecate cells; Figure S3). Dinoflagellate populations included known Group II Syndiniales hosts such as Alexandrium sp. (Nishitani et al., 1985, Jacobson, 1987, Sengco et al., 2003), Gonyaulax sp. (Kim et al., 2008, Siano et al., 2011), Gyrodinium sp. (Jacobson, 1987, Coats et al., 1996), Heterocapsa sp. (Kim, 2006, Chambouvet et al., 2008), Scrippsiella sp. (Coats et al., 1996, Chambouvet et al., 2011, Li et al., 2014), and Prorocentrum sp. (Maranda, 2001, Salomon et al., 2009). These findings suggest increased stratification observed in the summer months promoted proliferation of primarily dinoflagellate protist populations that are susceptible to Group II Syndiniales infections. 
Reads affiliated to Group II Syndiniales ASVs (Figure 2a) and parasitic infections (Figure 4) were most abundant when other dinoflagellate groups dominated. There are many factors that can contribute to Syndiniales cell concentrations and their representation in marker gene libraries across the time series, including infections of host populations, changes in gene content during different life stages of both the host and the parasite, and the short-lived survival of free-living spores. Syndinian diversity in Salt Pond also included sequences annotated to Groups I and III. Reads annotated to these groups were more abundant in early spring and from late May to mid-June, when communities were not dominated by dinoflagellates. Ciliates, thought to be the preferred host phylum for Group I Syndiniales (Guillou et al., 2008, Bachvaroff et al., 2012), were present in iTag libraries across the time series; however, there was no clear pattern of co-occurrence between individual ciliate and Group I Syndiniales ASVs. Reads annotated to Group III Syndiniales were more abundant in the late spring and, unlike reads annotated to Group II, were more highly represented in libraries collected at four meters depth than at two meters depth. There are no known hosts for Group III Syndiniales; therefore, observations of this taxon require additional investigation to better understand the ecological relevance in Salt Pond.

A seasonal impact on community composition was evident. Non-metric multidimensional scaling (NMDS, Figure 3) analysis of iTAG libraries showed that samples grouped closely by month, with no distinction between sampling depths. Environmental vectors fitted to the NMDS ordination indicated that temperature, salinity, dissolved oxygen, wind direction, maximum wind gust, and combined nitrate and nitrite concentrations were all significant factors structuring community composition. Among these, the most significant variables either directly enhanced seasonal stratification (e.g. temperature, salinity) or were the result of it (e.g. dissolved oxygen, nutrient concentrations). Previous research suggested stratification (Margalef et al., 1979) and diverse metabolic capabilities of dinoflagellates (Smayda and Reynolds, 2003, Kremp et al., 2008) promote dinoflagellate-dominated communities in coastal systems. In Salt Pond, intensifying stratification led to a shift in the protist community toward dinoflagellates in July, August, and September. 
Spatial and temporal variation in Group II Syndiniales abundances

Although ALV01 did not target all Salt Pond Group II ASVs (it covered 341 out of 440), it was

still useful for observing high resolution changes in abundance of members of this protist group in a coastal setting. Infections were sparse in the early spring, but as biomass increased and stratification intensified in the summer months, abundances and infections increased (Figure 4). Periodic infections coincided with alternating blooms and crashes of putative host taxa ASVs observed at near-monthly intervals. Infections often concentrated nearer to the surface and were more abundant at the two- and three-meters depths than at the 4m depth in July, August, and September, suggesting Group II Syndiniales in Salt Pond preferentially target thecate and athecate dinoflagellate hosts that were found in more oxic waters. Microscopy revealed common host morphotypes that were consistent with heterocapsids, scrippsielloids, and large athecate cells (Figure S3). These same infected morphotypes were also observed in samples from fourmeter depth, but were less abundant there, and may have been sinking cells from the surface. Consistent with previous studies, no ciliate morphotypes appeared to be infected by Group II syndinian parasites targeted by the ALV01 probe.

A lower proportion of infections observed at the four meters depth in the summer months (June to September) could be partially explained by abundant unidentified and possibly mixotrophic, green euglenoids $\left(\sim 10^{6}\right.$ cells/L) present in the lower oxygen samples (at 3 and $4 \mathrm{~m}$ depth), which were not infected by the Group II Syndiniales parasites. These euglenoids were not detected by our eukaryotic primers in the iTag libraries; therefore, a future study is needed to evaluate the role of these euglenoids in micro-oxic to anoxic marine systems like Salt Pond.

Infections lead to the release of dozens to hundreds of infective parasite spores per lysed host, which could be an important source of particulate organic matter for the local microbial community (Edgcomb, 2016). Group II Syndiniales dinospore concentrations were enumerated in samples collected from the $2 \mathrm{~m}$ depth around the July, August and September infection events, using the ALV01 CARD-FISH probe. Counts of dinospores were highest on the sample dates concurrent with the July and September infection peaks (Figure 4). These observations differ from previous studies that showed peaks in dinospore concentrations that lagged peaks in infections by several days. The lag is caused because spores are released from infections approximately 24 to 48 hours after initiation of an infection (Chambouvet et al., 2008, VeloSuarez et al., 2013). Given the 2- to 3-day interval between our sampling dates, it is possible that 
spore concentrations were higher than we observed in the day(s) following the infection events, on days that we did not sample. It is also possible that released spores were rapidly removed from the system by mortality, grazing, or hydrodynamic processes. No peak in dinospores was observed during the August infection event (Figure 4), which may indicate removal processes that exceeded production during this time period.

Cohorts highlight potential environmental and biological drivers of succession Four cohorts of co-occurring protist taxa, consisting of 284 ASV taxonomic bins annotated to the highest level possible (Table S3), were classified using the k-medoids clustering method. The timing and composition of these cohorts was compared to the environmental measurements and biological observations to generate new hypotheses about the factors influencing protist community structure and succession in Salt Pond.

The timing of the July and August infection events coincided with the presence of cohort one, which extended from early June to mid-August. Cohort one consisted of 31 species of dinoflagellates, including the genera Alexandrium sp., Gyrodinium sp., Heterocapsa sp., and Scrippsiella sp. These taxa are known to host Group II Syndiniales parasites and have been associated with reports of infections in diverse coastal systems including the Chesapeake Bay (Maryland, USA; Coats and Bockstahler, 1994, Coats et al., 1996), Thau Lagoon (Sete, France; Chambouvet et al., 2011b), Nauset Marsh system (Massachusetts, USA; Velo-Suarez et al., 2013), Penzé Estuary (Bretagne, France; Chambouvet et al., 2008) and the oligotrophic Mediterranean Sea (Siano et al., 2011). Morphologies of infected hosts are consistent with the aforementioned taxonomic groups in Salt Pond. Nine Group II Syndiniales clades were found to co-occur and included clades 16,39, and 44, which have no known hosts.

Infections in multiple dinoflagellate morphotypes and the mixed assemblage of Group II Syndiniales parasites obscured specific host-parasite infection dynamics within cohort one. Sequences annotated to Group II Clade 2 were abundant on July $3^{\text {rd }}$ and appeared to infect thecate dinoflagellate morphotypes, like Heterocapsa sp. and Scrippsiella sp., and small $(\sim 10 \mu \mathrm{m})$ athecate cells that could not be identified microscopically. Heterocapsid morphotypes (Figure S3 a-g) represented $17.76 \%$ of protists $(\sim 130,000$ cells/L), and of these cells, $4.72 \%$ were infected by Group II syndinian parasites. Scrippsielloid morphotypes (Figure S3 h-m) were 9.60\% of all protists enumerated ( $\sim 16,000$ cells/L), and of these cells, $16.52 \%$ were infected (Figure S4). 
Multiple host morphologies suggest that Clade 2 parasites are either generalists or the clade is composed of multiple species, collectively capable of infecting multiple protist hosts. Indeed, 51 ASV sequences are annotated to Clade 2 parasites, which suggests sub-clade variability (Cai et al., 2020). The August $13^{\text {th }}$ infection event involved mixed assemblages of both hosts and parasite clades. Notably, increased parasite diversity (Figure S4) was correlated with higher infection levels (Figure 4). This infection event impacted the largest fraction of the in-situ protist community $(2.56 \%)$ and was associated with multiple clades of Group II Syndiniales. Of the dominant morphotypes, $15.75 \%$ of the community was heterocapsid cells $(\sim 160,000$ cells/L) and $15.1 \%$ of cells with this morphology was infected. Clades 1, 2, 4, 5, 14, 22 and other unassigned Group II sequences were present in iTag libraries and may have contributed to observed infections of hosts with diverse morphologies (Figure S5). Putative hosts for clades 1, 2, 4, and 14 include Alexandrium sp. Gonyaulax sp., Gymnodinium sp., Heterocapsa sp., and Scrippsiella sp. (Coats et al., 1996, Chambouvet et al., 2008, Siano et al., 2011). Observed infections of small thecate and athecate cells suggest the host ranges of these parasites may include other taxa that cannot be easily identified morphologically (Figure S3 t-z). To date, Clades 5 and 22 have no identified hosts and our observations of these strains in Salt Pond underscore the technical challenges that remain when characterizing ecological niches for many syndinian parasites.

Syndinian parasite infections impacted the largest fraction of the protist community on August $13^{\text {th }}$. In addition, the protist community transitioned from dinoflagellate-dominated to diatom- and ciliate-dominated. Syndinian parasites can exert top-down pressure on their protist hosts and may stimulate microbial growth through the release of dissolved and particulate organic matter during host cell lysis (Guillou et al., 2008). Other studies of host-parasite systems have shown host-cell lysis contributes to increased nutrient availability and the growth of microbes in the surrounding water column (Salomon et al., 2009, Warren et al., 2010, Berdjeb et al., 2018, Frainer et al., 2018). Parasitism may have influenced the shift toward diatom genera in cohort two in mid-August, where lysis of host dinoflagellates contributed to nutrient hotspots, although other driving forces, such as temperature and nutrient shifts driven by intensified stratification, cannot be ruled out. Ammonium, nitrate and nitrite measurements collected during this transition do not indicate large fluxes in nitrogen concentrations. The diatom genera in cohort two differed from early spring diatoms (dominated by Thalassiosira, Pinnularia, and Leptocylindrus) and included the cosmopolitan and chain-forming taxa Chaetoceros, Navicula, 
and Skeletonema. Growth, chain-formation (in the case of Skeletonema and Chaetoceros) and photosynthetic activity of these diatoms are influenced by factors that include nutrient availability (e.g.. nitrate, phosphate, silicate; Karthikeyan et al., 2013, Liu et al., 2013, Zhao et al., 2014, Wang et al., 2020). The diatoms observed in cohort two did not appear to be parasitized by Group II Syndiniales targeted by the ALV01 probes.

Ciliate taxa were also abundant and diverse in cohort two, although no parasitism by Group II Syndiniales was observed in samples from our study. These included Frontonia, Aspidisca, and Strombidium. Parasitic relationships between ciliates and Group II Syndiniales have been hypothesized for coastal ciliate populations based on co-occurrence patterns (TorresBeltran et al., 2018). It is possible that ciliate species targeted by Group II Syndiniales were not present in Salt Pond, or similarly, that ciliates may have been infected by Syndiniales Group II clades that are not targeted by the CARD-FISH probe we used. Another possibility is that ciliates and Group II Syndiniales may co-occur in other datasets because of grazing and not parasitic interactions (Anderson and Harvey, 2020). Microscopy data before and after August 13 ${ }^{\text {th }}$ suggest free-living Syndiniales parasite spores were rapidly removed (Figure 4). Although we do not have direct evidence for increased grazing on parasite spores by the ciliates in cohort two, grazing may explain their rapid removal following this infection event.

Cohort three spanned from late April to late May and represented a period of time with very low infection prevalence based on microscopy, as well as relatively fewer Syndiniales sequences in marker gene libraries. The composition of cohort three included many species of chlorophytes and stramenopiles (Table S3). Of course, parasitism by other parasitic groups may also occur throughout the year. Chytrid fungi, known to infect diatoms (Peacock et al., 2014, Frenken et al., 2017, Kilias et al., 2020), were among cohort three. The community shifts from diatom-chytrid to dinoflagellate-Syndiniales co-occurrences documented in Salt Pond may represent population succession mediated by seasonal environmental triggers and/ or the effects of top-down control of Syndiniales and fungal parasitism on host populations.

The community shift toward cohort four occurred around September $14^{\text {th }}$, and was composed of recurring protist taxa including ciliates, chlorophytes, diatoms, MAST stramenopiles, and dinoflagellates that were present in both early spring and early fall. Cohort four was distinct from communities during the July and August infection events. Microscopic observations confirmed infected hosts included larger athecate dinoflagellates $(\sim 25 \mu \mathrm{m}$ size; 
Figure S3 n-s) compared to smaller athecate hosts and thecate hosts observed during July and August. The large athecate cell morphotype represented $25.21 \%$ of the protist community during the peak of the infection event, although cells were not heavily infected $(2.43 \%$ on September $14^{\text {th }}$; Figure S4). Cells with this morphology increased to $73.52 \%$ of the protist community by September $20^{\text {th }}$, suggesting the parasitic infections observed on September $14^{\text {th }}$ did not exert significant top-down pressure on this specific protist population. Cohort four included Group II Syndiniales clades $(26,30,32)$ that differed from the other cohorts, suggesting host selectivity and/or specific ecological niches. Abiotic factors, including cooler temperatures, shorter day lengths, increased vertical mixing, and nutrient availability are known to influence the periodicity of protists (Lawrence and Menden-Deuer, 2012, Simon et al., 2015) and may explain the appearance of cohort four communities both in spring and fall in Salt Pond, and distinct host/parasite assemblages.

\section{CONCLUSION}

Syndinian parasites are some of the most abundant and poorly-characterized protists across the global ocean, representing an ecological "black-box" in models of marine food webs and biogeochemical cycling. Molecular and microscopy observations from this high-resolution time series study of water samples from Salt Pond reveal both subtle and more prominent shifts in diversity of Group II Syndiniales as well as putative host populations in a coastal marine setting between March and October 2018. Infections were observed predominantly in small (10$25 \mu \mathrm{m}$ cell size) thecate and athecate dinoflagellate hosts throughout most of the time series, with larger infection events occurring in July, August, and September. Host morphologies and analyses of co-occurring protist taxa suggested unique host-parasite interactions during each infection event across the time series. Infection events coincided most often with dinoflagellatedominated protist communities that emerged as stratification intensified in Salt Pond; however, large blooms of individual dinoflagellate populations were not observed in this system. Salt Pond represents a different perspective on the activity of Syndiniales parasites, where infections occurred in host communities that were generally lower in abundance than the intensively studied one-species bloom scenario, but still able to propagate efficiently in complex protist communities. This pattern of host/parasite interaction may be more representative of many other marine environments where blooms of single taxa are not the general observation. This theory, 
and hypotheses regarding generalist versus specific host/parasite associations, will benefit from expanding microscopy studies to other sites and applying single cell genomic approaches.

\section{ACKNOWLEDGEMENTS}

The authors would like to thank David Beaudoin, Emily Maness, Sarah Lott, Emma Keeler, Rebecca Cox, and Carmine Riccardi for their assistance collecting and processing samples. Thank you to Dr. Jeff Donnelly and Ed Lott for donating boats for sample collection and the occasional rescue. Thank you to Dr. Sarah Hu for her guidance analyzing marker gene libraries and for determining patterns of co-occurring taxa. We thank Dr. Paraskevi Mara for her helpful comments on the manuscript and assistance in the field. This material is based upon work supported by the National Science Foundation Biological Oceanography OCE-1851012 to RG and VE, and by the National Science Foundation Graduate Research Fellowship under Grant No. 1745302. 


\section{REFERENCES}

Anderson, S.R. and Harvey, E.L., 2020. Temporal variability and ecological interactions of parasitic marine Syndiniales in coastal protist communities. Msphere 5(3).

Arístegui, J., Gasol, J.M., Duarte, C.M., Herndl, G.J., 2009. Microbial oceanography of the dark ocean's pelagic realm. Limnology and Oceanography, 54, pp.1501-1529.

Bachvaroff, T.R., Kim, S., Guillou, L., Delwiche, C. F., and Coats, D.W., 2012. Molecular diversity of the syndinean genus Euduboscquella based on single-cell PCR analysis. Applied Environmental Microbiology, 78, pp.334-345.

Balzano, S., Abs, E. and Leterme, S.C., 2015. Protist diversity along a salinity gradient in a coastal lagoon. Aquatic Microbial Ecology, 74(3), pp.263-277.

Bazylinski, D.A., Schlezinger, D.R., Howes, B.H., Frankel, R.B. and Epstein, S.S., 2000. Occurrence and distribution of diverse populations of magnetic protists in a chemically stratified coastal salt pond. Chemical Geology, 169, pp.319-328.

Berdjeb, L., Parada, A., Needham, D.M. and Fuhrman, J.A., 2018. Short-term dynamics and interactions of marine protist communities during the spring-summer transition. The ISME Journal, 12(8), pp.1907-1917.

Borchers, H.W., 2021. pracama: Practical Numerical Math Functions. R package version 2.3.3.

Bråte, J., Krabberød, A. K., Dolven, J. K., Ose, R. F., Kristensen, T., Bjørklund, K. R., and Shalchian-Tabrizi, K., 2012. Radiolaria associated with large diversity of marine alveolates. Protist, 163, pp.767-777.

Cachon, J., 1964. Contribution à l'étude des péridiniens parasites. Cytologie, cycles évolutifs. Annales des Sciences Naturelles Zoologies, 6, pp.1-158.

Cachon, J. and Cachon, M., 1970. Ultrastructure des Amoebophryidae (Péridiniens Duboscquodinida) II Systèmes atractophriens et microtubulaires; leur intervention dans la mitose. Protistologica, 6, pp.57-70.

Cai, R., Kayal, E., Alves-De-Souza, C., Bigeard, E., Corre, E., Jeanthon, C., Marie, D., Porcel, B.M., Siano, R., Szymczak, J. and Wolf, M., 2020. Cryptic species in the parasitic Amoebophrya species complex revealed by a polyphasic approach. Scientific Reports, 10(1), pp.1-11.

Carstensen, J., Klais, R. and Cloern, J.E., 2015. Phytoplankton blooms in estuarine and coastal waters: Seasonal patterns and key species. Estuary and Coastal Shelf Science, 162, pp.98-109.

Chambouvet, A., Morin, P., Marie, D., and Guillou, L., 2008. Control of toxic marine dinoflagellate blooms by serial parasitic killers. Science, 322, pp.1254-1257. 
Chambouvet, A., Alves-de-Souza, C., Cueff, V., Marie, D., Karpov, S., and Guillou, L., 2011. Interplay between the parasite Amoebophrya sp. (Alveolata) and the cyst formation of the red tide dinoflagellate Scrippsiella trochoidea. Protist, 162(4), pp.637-649.

Chambouvet, A., Laabir, M., Sengco, M., Vaquer, A., and Guillou, L. (2011)b Genetic diversity of Amoebophryidae (Syndiniales) during Alexandrium catenella/tamarense (Dinophyceae) blooms in the Thau lagoon (Mediterranean Sea, France). Research in Microbiology, 162, pp.959968.

Christaki, U., Genitsaris, S., Monchy, S., Li, L.L., Rachik, S., Breton, E. and Sime-Ngando, T., 2017. Parasitic eukaryotes in a meso-eutrophic coastal system with marked Phaeocystis globosa blooms. Frontiers in Marine Science, 4, pp.416.

Cleary, A.C. and Durbin, E.G., 2016. Unexpected prevalence of parasite 18S rDNA sequences in winter among Antarctic marine protists. Journal of Plankton Research, 38(3), pp.401-417.

Coats, D.W. and Bockstahler, K.R., 1994. Amoebophrya ceratii in Chesapeake Bay populations of Gymnodinium sanguineum. Journal of Eukaryotic Microbiology, 41, pp.586-593.

Coats, D.W., Adam, E.J., Gallegos, C.L. and Hedrick, S., 1996. Parasitism of photosynthetic dinoflagellates in a shallow subestuary of Chesapeake Bay, USA. Aquatic Microbial Ecology, 11(1), pp.1-9.

Coats, D.W. and Park M.G., 2002. Parasitism of photosynthetic dinoflagellates by three strains of Amoebophrya (Dinophyta): parasite survival, infectivity, generation time, and host specificity. Journal of Phycology, 38(3), pp.520-528.

Coenen, A.R., Hu, S.K., Luo, E., Muratore, D., and Weitz, J.W., 2019. A Primer for Microbiome Time-Series Analysis. https://github.com/shu251/analyzing microbiome timeseries

De Vargas, C., Audic, S., Henry, N., Decelle, J., Mahé, F., Logares, R., et al., 2015. Eukaryotic plankton diversity in the sunlit ocean. Science, 348(6237).

Duret, M.T., Pachiadaki, M.G., Stewart, F.J., Sarode, N., Christaki, U., Monchy, S., Srivastava, A., and Edgcomb, V.P., 2015. Size-fractionated diversity of eukaryotic microbial communities in the Eastern Tropical North Pacific oxygen minimum zone. FEMS Microbiology Ecology, 91(5).

Edgcomb, V.P., Orsi, W., Bunge, J., Jeon, S.-O., Christen, R., Leslin, C., et al., 2011. Protistan microbial observatory in the Cariaco Basin, Caribbean. I. Pyrosequencing vs. Sanger insights into species richness. The ISME Journal, 5, pp.1344-1356.

Edgcomb, V.P., 2016. Marine protist associations and environmental impacts across trophic levels in the twilight zone and below. Current Opinions in Microbiology, 31, pp.169-175. 
Frainer, A., McKie, B.G., Amundsen, P.A., Knudsen, R. and Lafferty, K.D., 2018. Parasitism and the biodiversity-functioning relationship. Trends in Ecology and Evolution, 33(4), pp.260268.

Frenken, T., Alacid, E., Berger, S. A., Bourne, E. C., Gerphagnon, M., Grossart, H. P., et al., 2017. Integrating chytrid fungal parasites into plankton ecology: research gaps and needs.

Environmental Microbiology, 19(10), pp.3802-3822.

Galluzzi, L., Bertozzini, E., Penna, A., Perini, F., Garcés, E. and Magnani, M., 2010. Analysis of rRNA gene content in the Mediterranean dinoflagellate Alexandrium catenella and Alexandrium taylori: implications for the quantitative real-time PCR-based monitoring methods. Journal of Applied Phycology, 22(1), pp.1-9.

Giblin, A.E., Tobias, C.R., Song, B., Weston, N., Banta, G.T., Rivera-Monroy, V.H., 2013. The importance of dissimilatory nitrate reduction to ammonium (DNRA) in the nitrogen cycle of coastal ecosystems. Oceanography, 26(3), pp.124-131.

Gong, W. and Marchetti, A., 2019. Estimation of 18S gene copy number in marine eukaryotic plankton using a next-generation sequencing approach. Frontiers in Marine Science, 6, pp.219.

Guillou, L., Viprey, M., Chambouvet, A., Welsh, R., Kirkham, A.R., Massana, R., Scanlan, D.J., and Worden, A.Z., 2008. Widespread occurrence and genetic diversity of marine parasitoids belonging to Syndiniales (Alveolata). Environmental Microbiology, 10, pp.3349-3365.

Frank E Harrell Jr (2021). Hmisc: Harrell Miscellaneous. R package version 4.6-0. https://CRAN.R-project.org/package $=$ Hmisc

Jacobson, D.M., 1987. The ecology and feeding biology of thecate heterotrophic dinoflagellates. Doctoral dissertation, Woods Hole Oceanographic Institution.

Jephcott, T.G., Alves-de-Souza, C., Gleason, F.H., van Ogtrop, F.F., Sime-Ngando, T., Karpov, S.A., and Guillou, L., 2016. Ecological impacts of parasitic chytrids, syndiniales and perkinsids on populations of marine photosynthetic dinoflagellates. Fungal Ecology, 19, pp.47-58.

Jin, J., Liu, S.M., Ren, J.L., Liu, C.G., Zhang, J. and Zhang, G.L., 2013. Nutrient dynamics and coupling with phytoplankton species composition during the spring blooms in the Yellow Sea. Deep Sea Research Part II: Topical Studies in Oceanography, 97, pp.16-32.

Karthikeyan, P., Manimaran, K., Sampathkumar, P. and Rameshkumar, L., 2013. Growth and nutrient removal properties of the diatoms, Chaetoceros curvisetus and C. simplex under different nitrogen sources. Applied Water Science, 3(1), pp.49-55.

Kilias, E.S., Junges, L., Šupraha, L., Leonard, G., Metfies, K. and Richards, T.A., 2020. Chytrid fungi distribution and co-occurrence with diatoms correlate with sea ice melt in the Arctic Ocean. Communications Biology, 3(1), pp.1-13. 
Kim, S., 2006. Patterns in host range for two strains of Amoebophrya (Dinophyta) infecting thecate dinoflagellates: Amoebophrya spp. ex Alexandrium affine and ex Gonyaulax polygramma. Journal of Phycology, 42(6), pp.1170-1173.

Kim, S., Park, M.G., Kim, K.Y., Kim, C.H., Yih, W., Park, J.S. and Coats, D.W., 2008. Genetic diversity of parasitic dinoflagellates in the genus Amoebophrya and its relationship to parasite biology and biogeography. Journal of Eukaryotic Microbiology, 55(1), pp.1-8.

Kremp, A., Tamminen, T. and Spilling, K., 2008. Dinoflagellate bloom formation in natural assemblages with diatoms: nutrient competition and growth strategies in Baltic spring phytoplankton. Aquatic Microbial Ecology, 50(2), pp.181-196.

Lawrence, C. and Menden-Deuer, S., 2012. Drivers of protistan grazing pressure: seasonal signals of plankton community composition and environmental conditions. Marine Ecology Progress Series, 459, pp.39-52.

Li, C., Song, S., Liu, Y. and Chen, T., 2014. Occurrence of Amoebophrya spp. infection in planktonic dinoflagellates in Changjiang (Yangtze River) Estuary, China. Harmful Algae, 37 , pp.117-124.

Lin, S., 2011. Genomic understanding of dinoflagellates. Research in Microbiology, 162(6), pp.551-569.

Liu, Y., Song, X., Cao, X. and Yu, Z., 2013. Responses of photosynthetic characters of Skeletonema costatum to different nutrient conditions. Journal of Plankton Research, 35(1), pp.165-176.

López-García, P., Rodríguez-Valera, F., Pedrós-Alió, C., Moreira, D., 2001. Unexpected diversity of small eukaryotes in deep-sea Antarctic plankton. Nature, 409, pp.603-607.

Love, M.I., Huber, W., and Anders, S., 2014. Moderated estimation of fold change and dispersion for RNA-seq data with DESeq2. Genome Biology, 15(550).

Maechler, M., Rousseeuw, P., Struyf, A., Hubert, M., and Hornik, K., 2021. cluster: Cluster Analysis Basics and Extensions. R package version 2.1.2.

Mallin, M.A., Paerl, H.W. and Rudek, J., 1991. Seasonal phytoplankton composition, productivity and biomass in the Neuse River estuary, North Carolina. Estuary and Coastal Shelf Science, 32(6), pp.609-623.

Maranda, L., 2001. Infection of Prorocentrum minimum (Dinophyceae) by the parasite Amoebophrya sp.(Dinoflagellata). Journal of Phycology, 37(2), pp.245-248.

Margalef, R., Estrada, M., and Blasco, D., 1979. Functional morphology of organisms involved in red tides, as adapted to decaying turbulence. In: Taylor D, Seliger H, editors. Toxic Dinoflagellate Blooms. New York: Elsevier. pp. 89-94. 
Massana, R., Balagué, V., Guillou, L., and Pedros-Alio, C., 2004. Picoeukaryotic diversity in an oligotrophic coastal site studied by molecular and culturing approaches. FEMS Microbiology Ecology, 50(3), pp.231-243.

Miller, J.J., Delwiche, C.F., and Coats, D.W., 2012. Ultrastructure of Amoebophrya sp. and its changes during the course of infection. Protist, 163, pp.720-745.

Moskowitz, B.M., Bazylinski, D.A., Egli, R., Frankel, R.B. and Edwards, K.J., 2008. Magnetic properties of marine magnetotactic bacteria in a seasonally stratified coastal pond (Salt Pond, MA, USA). Geophysical Journal International, 174(1), pp.75-92.

Nishitani, L., Erickson, G., and Chew, K.K., 1985. Role of the parasitic dinoflagellate Amoebophrya ceratii in control of Gonyaulax catenella populations. Toxic Dinoflagellates, pp.225-230.

Not, F., Gausling, R., Azam, F., Heidelberg, J.F., and Worden, A.Z., 2007. Vertical distribution of picoeukaryotic diversity in the Sargasso Sea. Environmental Microbiology, 9, pp.1233-1252.

Not, F., del Campo, J., Balagué, V., de Vargas, C. and Massana, R., 2009. New insights into the diversity of marine picoeukaryotes. PloS One, 4(9), e7143.

Orsi, W., Y.C. Song, S. Hallam, and Edgcomb, V.P., 2012. Effect of oxygen minimum zone formation on communities of marine protists. The ISME Journal, 6(8), pp.1586-1601.

Pawlowski, J., Audic, S., Adl, S., Bass, D., Belbahri, L., Berney, C., et al., 2012. CBOL protist working group: barcoding eukaryotic richness beyond the animal, plant, and fungal kingdoms. PLoS Biology, 10(11), e1001419.

Peacock, E.E., Olson, R.J. and Sosik, H.M., 2014. Parasitic infection of the diatom Guinardia delicatula, a recurrent and ecologically important phenomenon on the New England Shelf. Marine Ecology Progress Series, 503, pp.1-10.

Pernice, M.C., Logares, R., Guillou, L. and Massana, R., 2013. General patterns of diversity in major marine microeukaryote lineages. PLoS One, 8(2), e57170.

Pernice, M.C., Giner, C.R., Logares, R., Perera-Bel, J., Acinas, S.G., Duarte, C.M., Gasol, J.M., and Massana, R., 2015. Large variability of bathypelagic microbial eukaryotic communities across the world's oceans. The ISME Journal, 10, pp.945-958.

Prokopowich, C.D., Gregory, T.R. and Crease, T.J., 2003. The correlation between rDNA copy number and genome size in eukaryotes. Genome, 46(1), pp.48-50.

Salomon, P.S., Granéli, E., Neves, M.H. and Rodriguez, E.G., 2009. Infection by Amoebophrya spp. parasitoids of dinoflagellates in a tropical marine coastal area. Aquatic Microbial Ecology, 55(2), pp.143-153. 
Sassenhagen, I., Irion, S., Jardillier, L., Moreira, D. and Christaki, U., 2020. Protist interactions and community structure during early autumn in the Kerguelen region (Southern Ocean). Protist, 171(1), 125709.

Scholz, B., Guillou, L., Marano, A. V., Neuhauser, S., Sullivan, B. K., Karsten, U., et al., 2016. Zoosporic parasites infecting marine diatoms-a black box that needs to be opened. Fungal Ecology, 19, pp.59-76.

Sengco, M.R., Coats, D.W., Popendorf, K.J., Erdner, D.L., Gribble, K.E. and Anderson, D.M., 2003. Biological and phylogenetic characterization of Amoebophrya sp. ex Alexandrium tamarense. In Second Symposium on Harmful Marine Algae in the US, Abstract (Vol. 57).

Siano, R., Alves-de-Souza, C., Foulon, E., Bendif, El M. Simon, N., Guillou, L., and Not, F., 2011. Distribution and diversity of Amoebophryidae parasites across oligotrophic waters of the Mediterranean Sea. Biogeosciences, 8, pp.267-278.

Silva, A., Palma, S., Oliveira, P.B. and Moita, M.T., 2009. Composition and interannual variability of phytoplankton in a coastal upwelling region (Lisbon Bay, Portugal). Journal of Sea Research, 62(4), pp.238-249.

Simmons, S.L., Sievert, S.M., Frankel, R.B., Bazylinski, D.A. and Edwards, K.J., 2004. Spatiotemporal distribution of marine magnetotactic bacteria in a seasonally stratified coastal salt pond. Applied Environmental Microbiology, 70(10), pp.6230-6239.

Simmons, S.L., Bazylinski, D.A. and Edwards, K.J., 2007. Population dynamics of marine magnetotactic bacteria in a meromictic salt pond described with qPCR. Environmental Microbiology, 9(9), pp.2162-2174.

Simon, M., López-García, P., Deschamps, P., Moreira, D., Restoux, G., Bertolino, P. and Jardillier, L., 2015. Marked seasonality and high spatial variability of protist communities in shallow freshwater systems. The ISME Journal, 9(9), pp.1941-1953.

Skovgaard, A., Massana, R., Balague, V. and Saiz, E., 2005. Phylogenetic position of the copepod-infesting parasite Syndinium turbo (Dinoflagellata, Syndinea). Protist, 156(4), 413-423.

Skovgaard, A., 2014. Dirty tricks in the plankton: Diversity and role of marine parasitic protists. Acta Protozoologica, 53, pp.51-62.

Smayda, T.J. and Reynolds, C.S., 2003. Strategies of marine dinoflagellate survival and some rules of assembly. Journal of Sea Research, 49(2), pp.95-106.

Song, B., Lisa, J.A. and Tobias, C.R., 2014. Linking DNRA community structure and activity in a shallow lagoonal estuarine system. Frontiers in Microbiology, 5, pp.460. 
Spilling, K., Olli, K., Lehtoranta, J., Kremp, A., Tedesco, L., Tamelander, T., Klais, R., Peltonen, H. and Tamminen, T., 2018. Shifting diatom - dinoflagellate dominance during spring bloom in the Baltic Sea and its potential effects on biogeochemical cycling. Frontiers in Marine Science, 5, pp.327.

Stentiford, G.D. and Shields, J.D., 2005. A review of the parasitic dinoflagellates Hematodinium species and Hematodinium-like infections in marine crustaceans. Diseases of Aquatic Organisms, 66(1), pp.47-70.

Stoecker, D. K., Hansen, P. J., Caron, D. A., and Mitra, A., 2017. Mixotrophy in the marine plankton. Annual Review of Marine Science, 9, pp.311-335.

Sverdrup, H.U., 1953. On conditions for the vernal blooming of phytoplankton. Journal du Conseil International pour l'Exploration de la Mer, 18(3), pp.287-295.

Torres-Beltran, M., Sehein, T., Pachiadaki, M.G., Hallam, S.J. and Edgcomb, V., 2018. Protistan parasites along oxygen gradients in a seasonally anoxic fjord: a network approach to assessing potential host-parasite interactions. Deep Sea Research Part II: Topical Studies in Oceanography, 156, pp.97-110.

Trigueros, J.M. and Orive, E., 2001. Seasonal variations of diatoms and dinoflagellates in a shallow, temperate estuary, with emphasis on neritic assemblages. Hydrobiologia, 444(1), pp.119-133.

Velo-Suárez L., Brosnahan, M. L., Anderson, D. M., and McGillicuddy, D. J. Jr., 2013. A quantitative assessment of the role of the parasite Amoebophrya in the termination of Alexandrium fundyense blooms within a small coastal embayment. PLoS One, 8(12), e81150.

Venables, W.N. and Ripley, B.D., 2002. Modern Applied Statistics with S, Fourth edition. Springer, New York. ISBN 0-387-95457-0

Wakeham, S.G., Howes, B.L. and Dacey, J.W., 1984. Dimethyl sulphide in a stratified coastal salt pond. Nature, 310, pp.770-772.

Wakeham, S.G., Howes, B.L., Dacey, J.W.H., Schwarzenbach, R.P. and Zeyer, J., 1987. Biogeochemistry of dimethylsulfide in a seasonally stratified coastal salt pond. Geochimica et Cosmochimica Acta, 51, pp.1675-1684.

Wang, H., Chen, F., Mi, T., Liu, Q., Yu, Z. and Zhen, Y., 2020. Responses of marine diatom Skeletonema marinoi to nutrient deficiency: programmed cell death. Applied Environmental Microbiology, 86(3).

Warren, C.P., Pascual, M., Lafferty, K.D. and Kuris, A.M., 2010. The inverse niche model for food webs with parasites. Theoretical Ecology, 3(4), pp.285-294. 
Yoshie, N., Yamanaka, Y., Kishi, M.J. and Saito, H., 2003. One dimensional ecosystem model simulation of the effects of vertical dilution by the winter mixing on the spring diatom bloom. Journal of Oceanography, 59(5), pp.563-571.

Zemmelink, H.J., Houghton, L., Frew, N.M. and Dacey, J.W.H., 2006. Dimethylsulfide and major sulfur compounds in a stratified coastal salt pond. Limnology and Oceanography, 51(1), pp.271-279.

Zhao, X., Pang, S., Liu, F., Shan, T. and Li, J., 2014. Biological identification and determination of optimum growth conditions for four species of Navicula. Acta Oceanologica Sinica, 33(8), pp.111-118.

Zhou, Y., Zhang, Y., Li, F., Tan, L. and Wang, J., 2017. Nutrients structure changes impact the competition and succession between diatom and dinoflagellate in the East China Sea. Science of the Total Environment, 574, pp.499-508. 


\section{FIGURES}
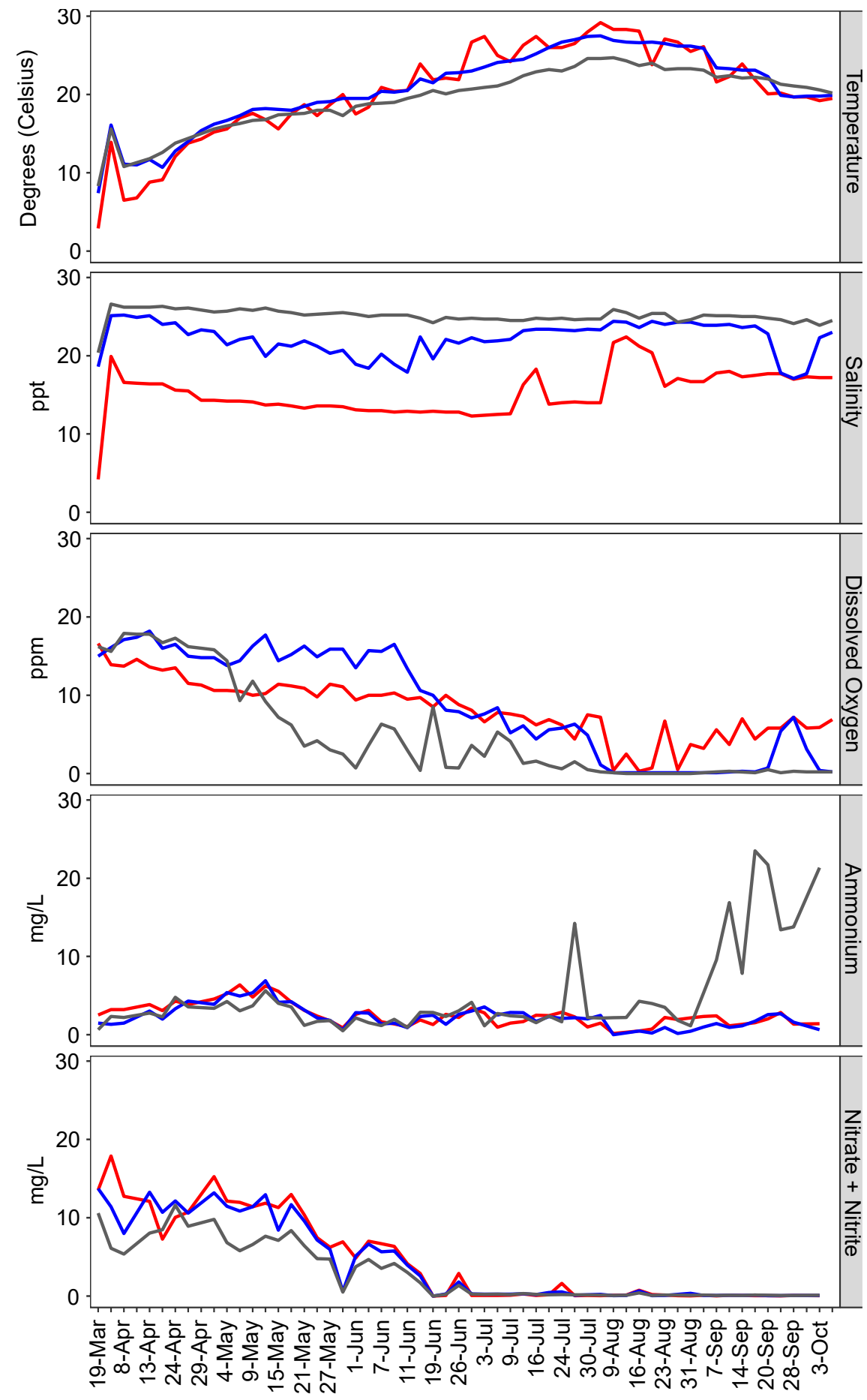

Figure 1. Abiotic variables measured across the time series from the three sampling depths.

Temperature, salinity, and dissolved oxygen were measured by hand-held YSI (YSI Pro2030, Ohio, USA). Water chemistry samples for ammonium and nitrate and nitrite were filtered and analyzed on an O.I. Analytical Flow Solutions IV auto analyzer. 
a.

b.
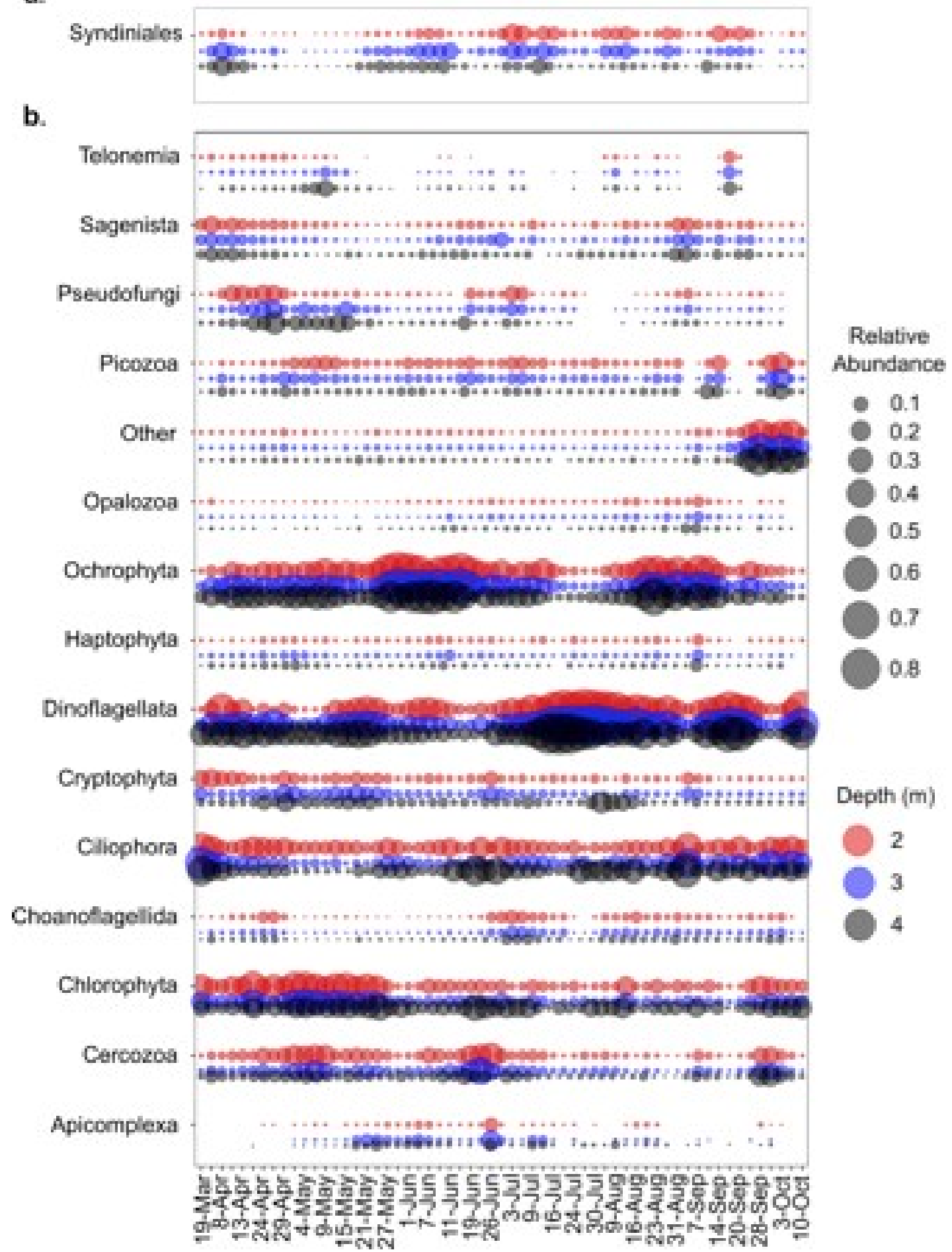

Figure 2. Relative abundances of reads annotated to a) Syndiniales and b) phyla in 18S rRNA marker gene libraries collected across the times series. Reads annotated to unassigned alveolates,

Perkinsea, Amoebozoa, Apusozoa, unassigned Archaeplastida, Streptophyta, Discoba, Centroheliozoa, Katablepharidophyta, unassigned opisthokonts, fungi, Mesomycetozoa, and unassigned stramenopiles are included in "Other." 


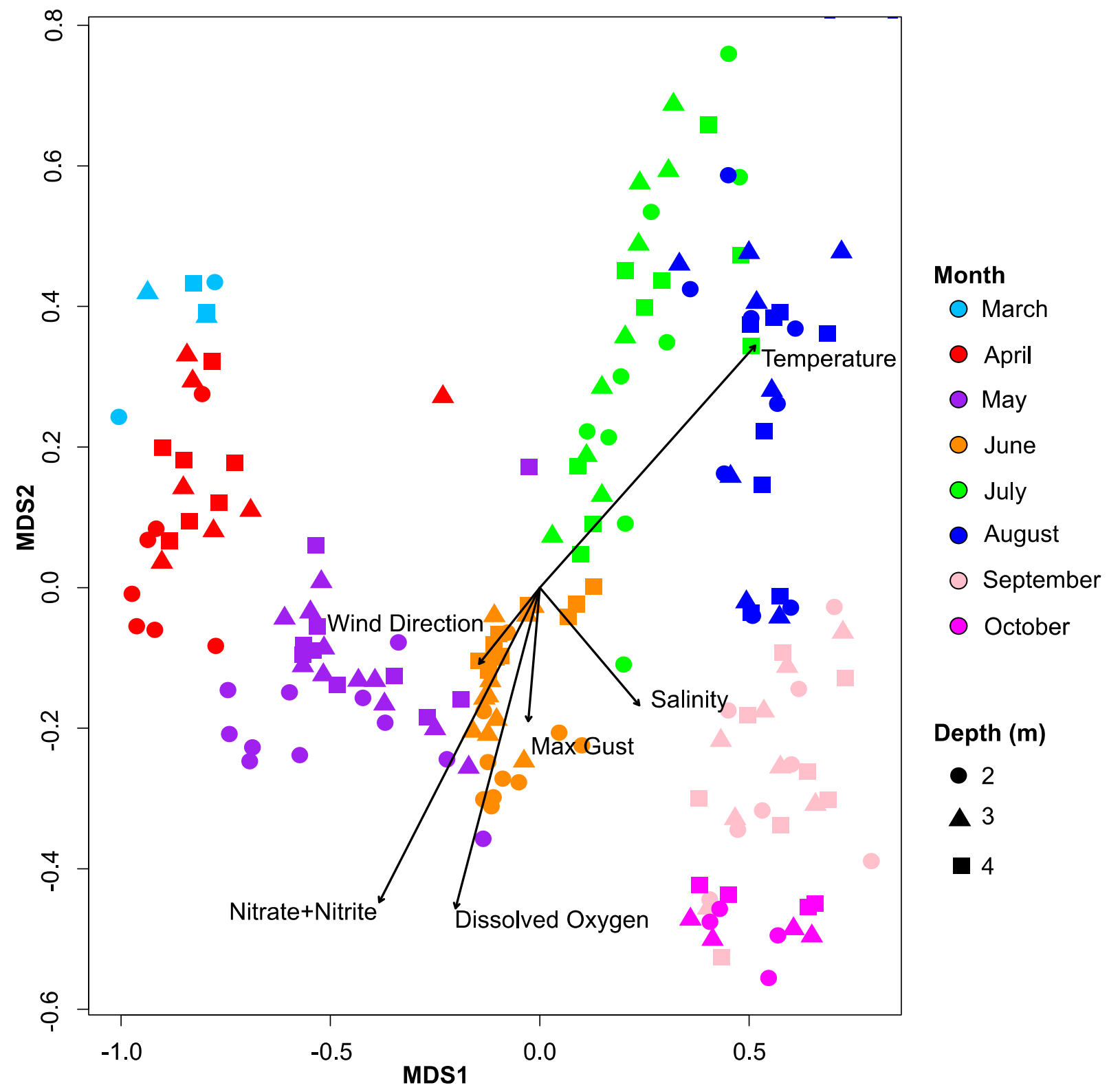

Figure 3. A nonmetric multidimensional scaling (NMDS) plot of 18S rRNA marker gene libraries collected across the time series and three depths. The distance matrix was constructed using Bray-Curtis dissimilarity. Vectors indicating significant correlations with environmental variables are displayed $(\mathrm{p}<0.05)$. 


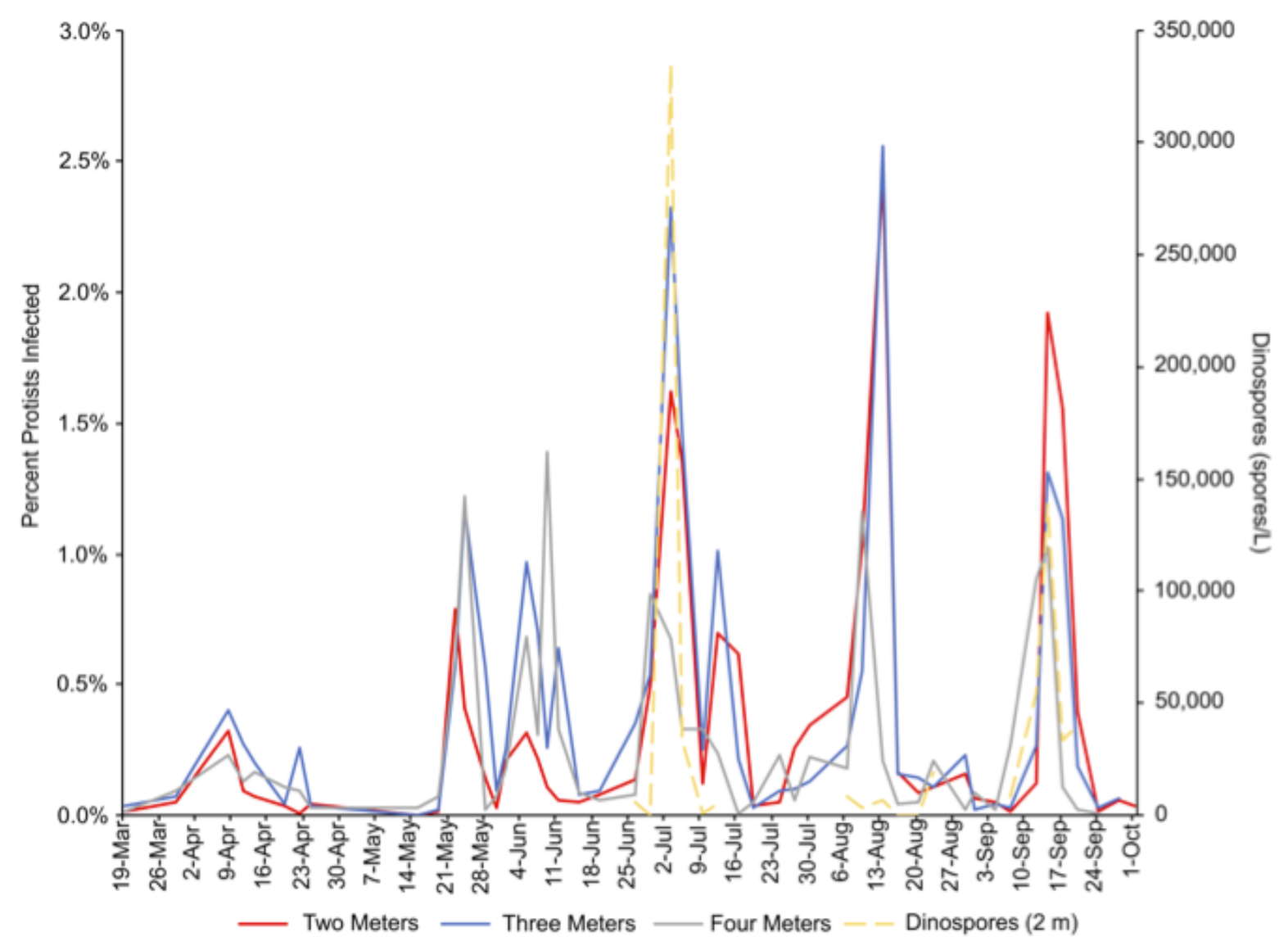

Figure 4. Group II Syndiniales infection prevalence and free-living spore concentrations labeled using the ALV01 CARD-FISH probe and enumerated by epifluorescent microscopy. Fractions of protists infected from the three sampling depths across the time series are presented (solid lines, primary axis) in addition to the concentration of parasite spores collected at two meters during the infection peaks in July, August, and September (dashed line, secondary axis). 


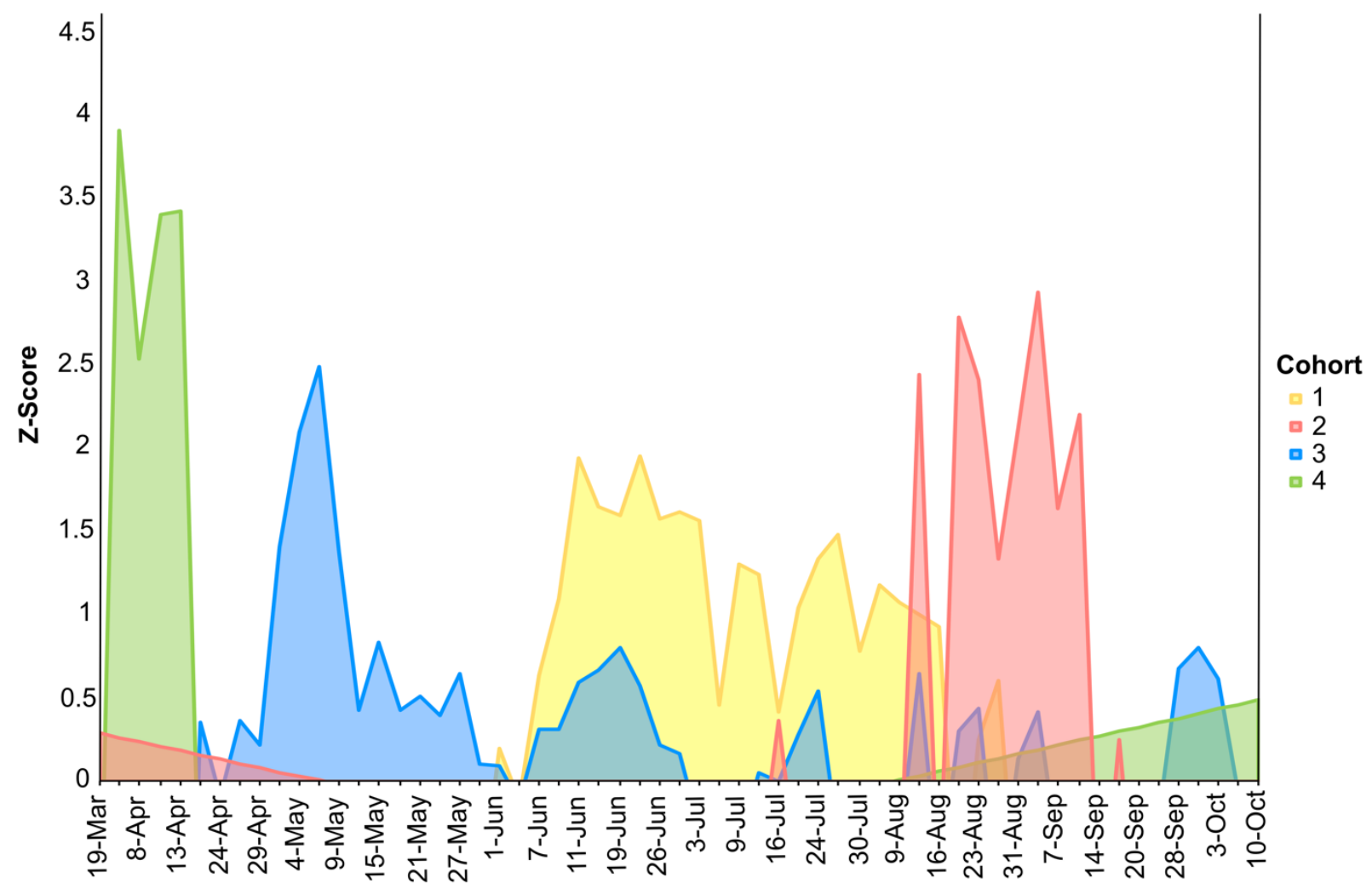

Figure 5. The summed z-scores show the succession of eukaryotic cohorts across the time series based on the read abundances of taxa within each cohort. The z-score for each taxon was calculated as the difference between the observed and mean number of reads divided by the standard deviation and described the frequency of reads that differed from the averaged baseline. Positive z-scores described when taxa from the cohorts were present at two meters. 


\section{SUPPLEMENTARY FIGURES}

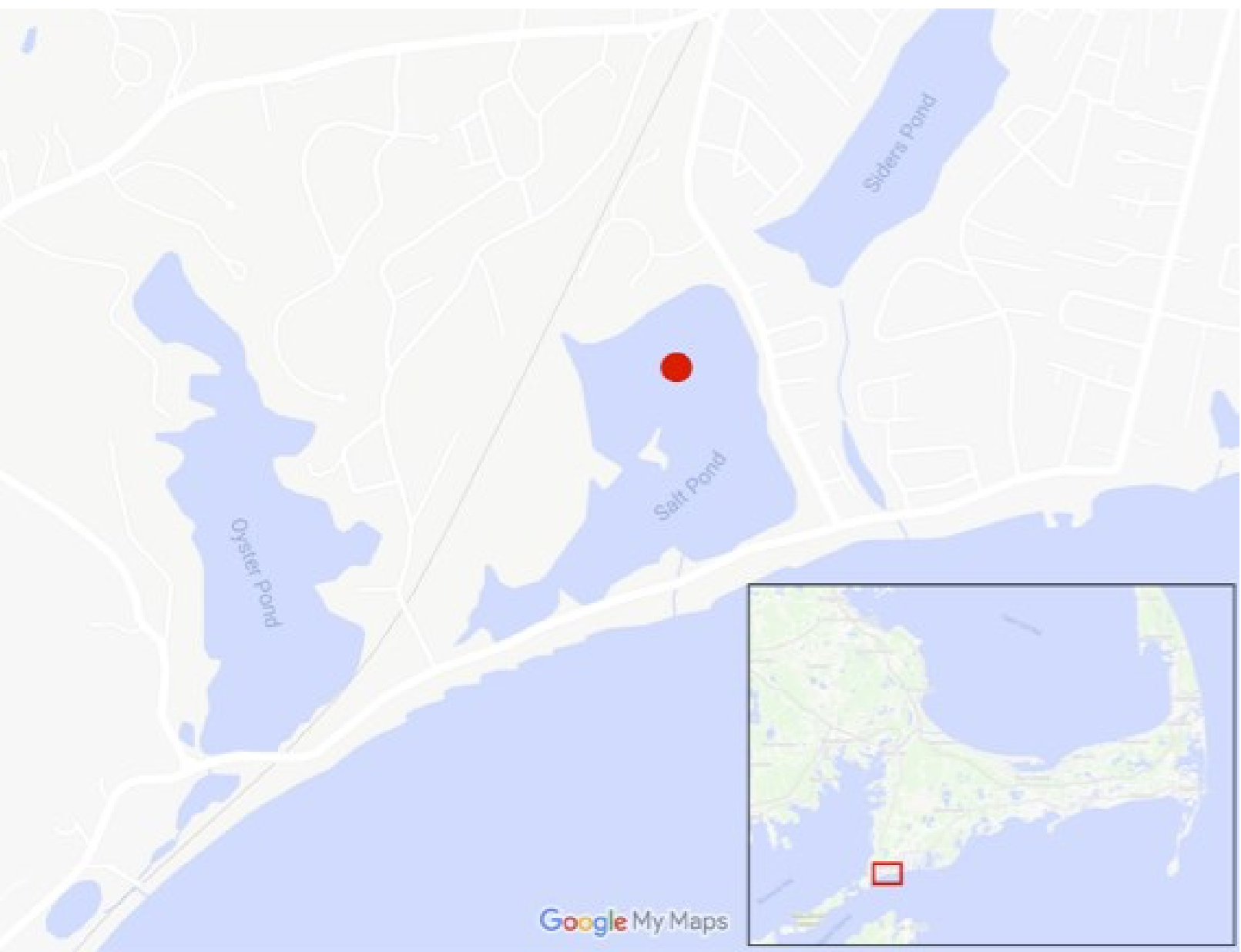

Figure S1. A map displaying the sampling point and the location of Salt Pond (Falmouth, MA; $41.5430^{\circ} \mathrm{N}, 70.6269^{\circ} \mathrm{W}$ ). 


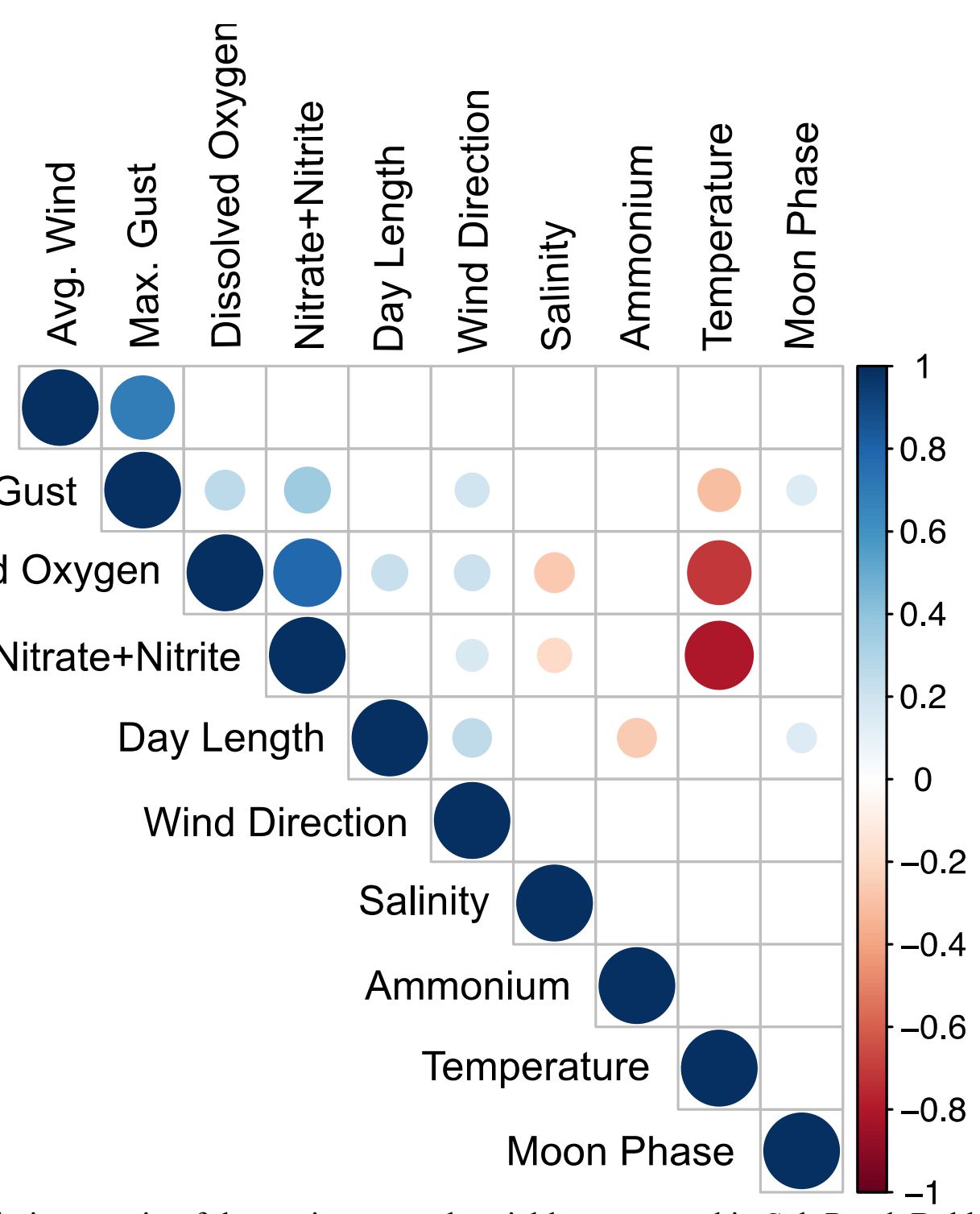

Figure S2. Correlation matrix of the environmental variables measured in Salt Pond. Bubble color and size are proportional to the correlation coefficients. Bubbles are only presented for correlated variables with significance values $\mathrm{p}<0.05$. 

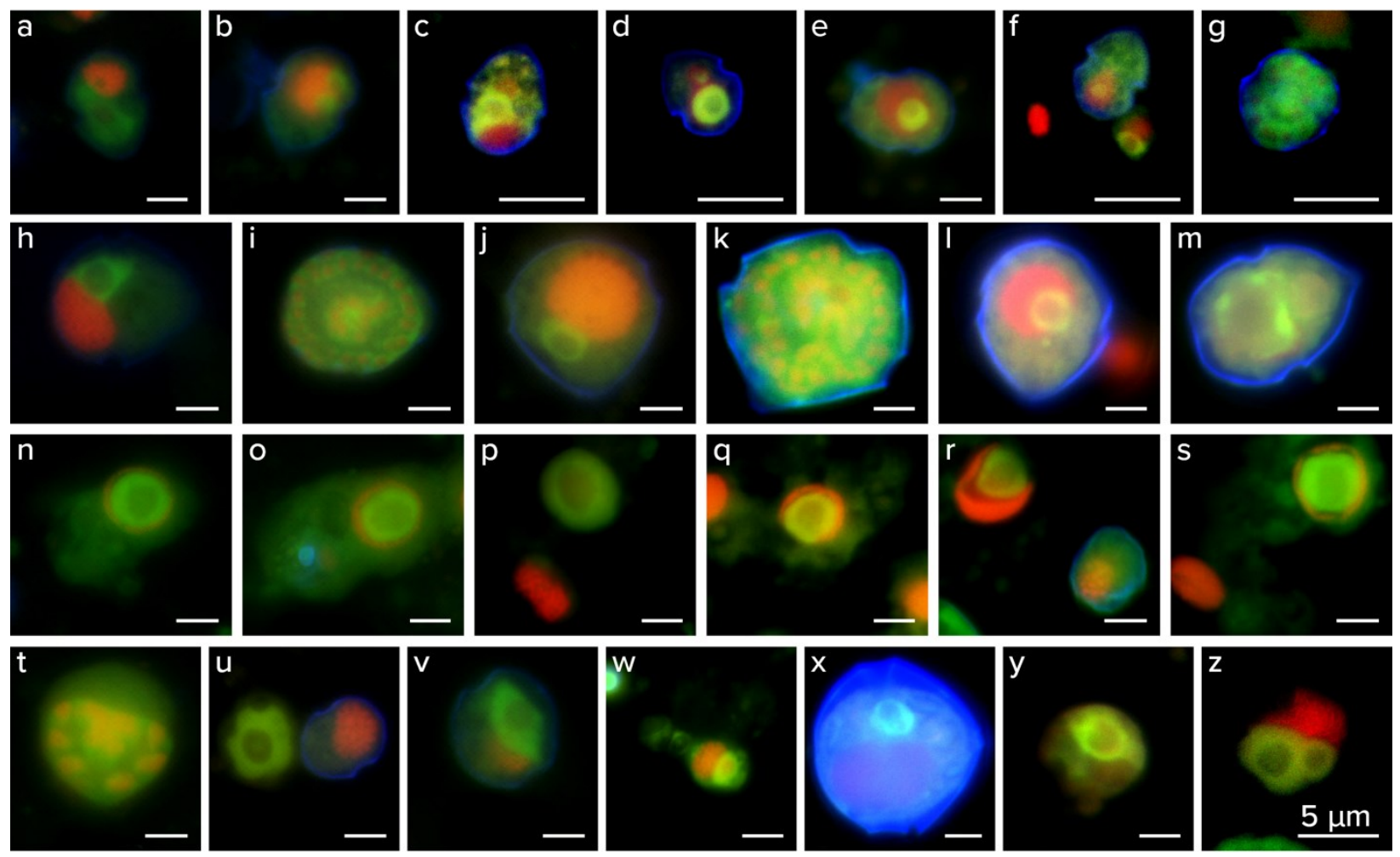

Figure S3. A selection of infected cells imaged during the three larger infection events. Parasites are labeled with the CARD-FISH probe ALV01 and stained green when observed with a FITC filter set. Host theca are stained with Calcofluor when present and blue when visualized with a DAPI filter. Both host and parasite nuclei are stained with propidium iodide and are red when viewed with a Cy3 filter set. (a-g) cells with heterocapsid morphology, (h-m) cells with scrippsielloid morphology, (n-s) cells with shared athecate morphology, and (t-z) other interesting, but less common, infection morphotypes. All scale bars denote $5 \mu \mathrm{m}$. 

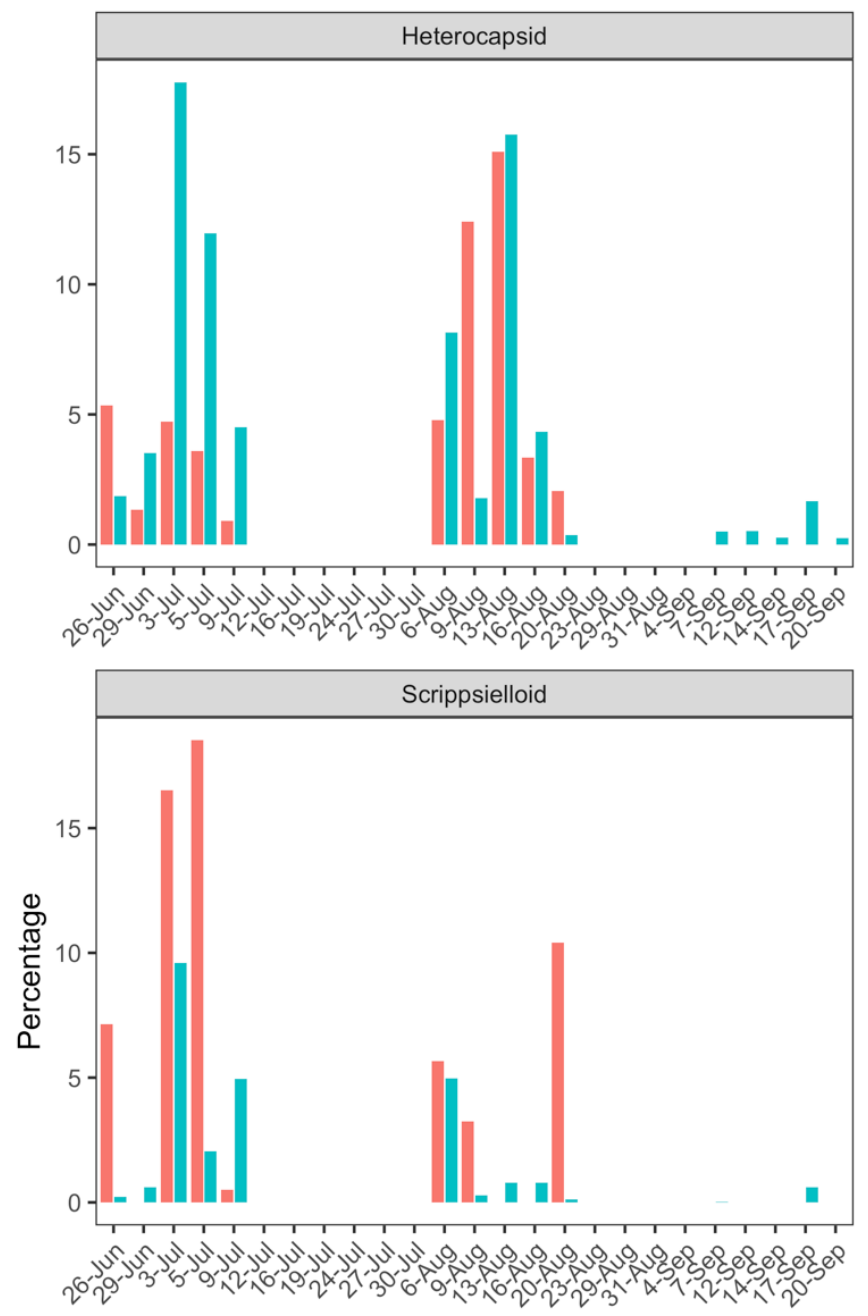

$$
\begin{aligned}
& \text { Type } \\
& \text { Percent Infected } \\
& \text { Percent Morphotype }
\end{aligned}
$$

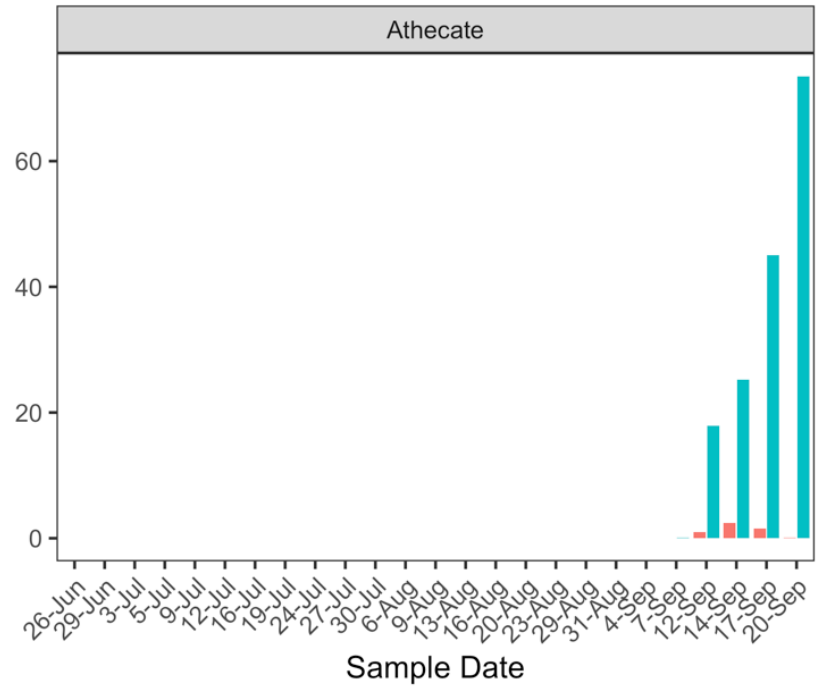

Figure S4. Group II Syndiniales infection prevalence in three host morphotypes present during infection events in July, August, and September. Infections were detected by CARD-FISH with the Group II Syndiniales probe, ALV01, and epifluorescent microscopy. 


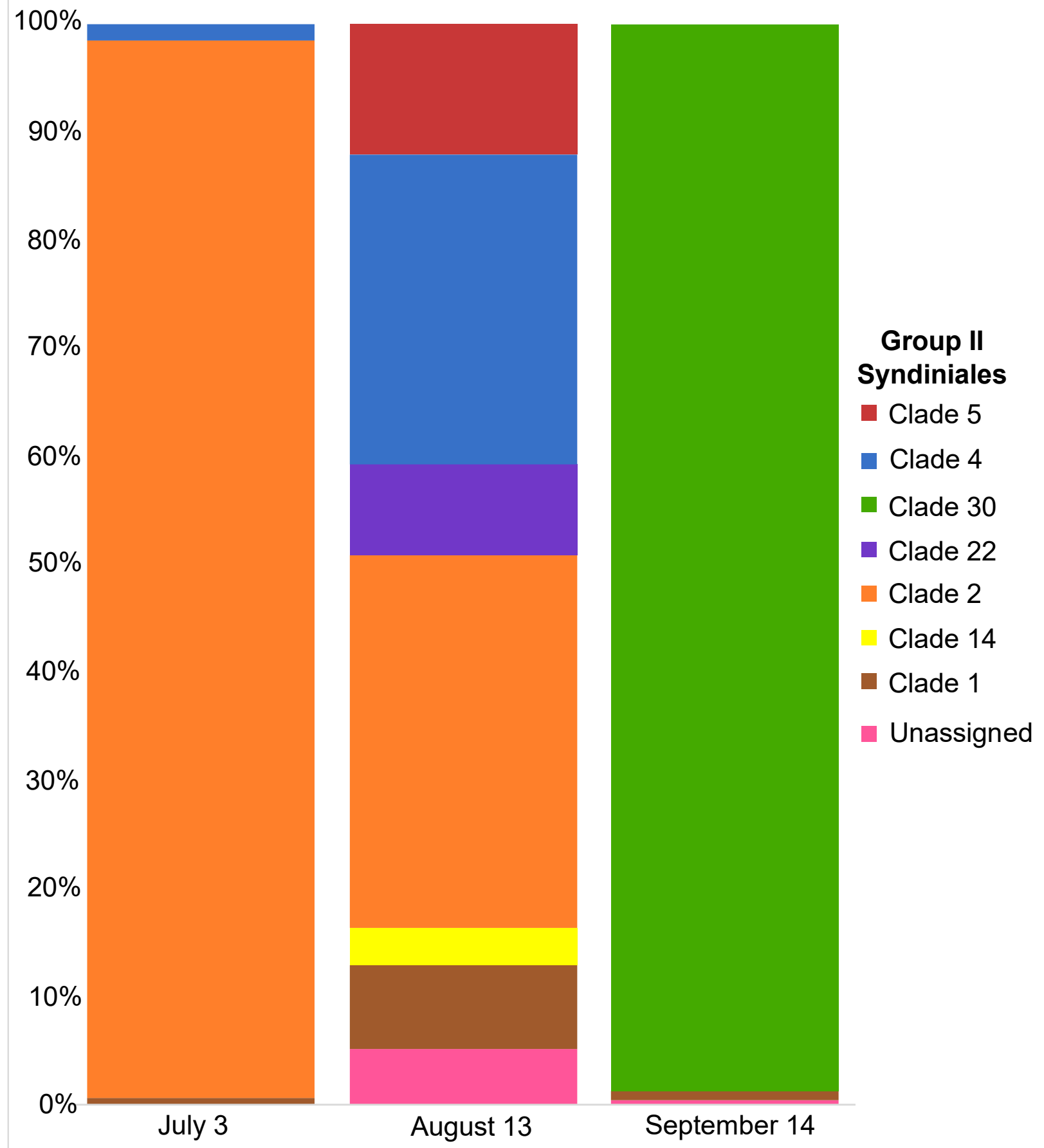

Figure S5. The relative abundance of Group II Syndiniales clade reads at two meters during three infection events. 


\section{SUPPLEMENTARY TABLE LEGENDS}

Table S1. A summary of the environmental parameters and water column chemistry measured for each sample cast. Sample identifiers correspond to the date, followed by the cast (C) replicate number and the depth ( $D ; 1=2$ meters, $2=3$ meters, $3=4$ meters).

Table S2. Raw read counts from all ASVs generated by the dada2 pipeline and annotated using the PR2 database. Sample identifiers correspond to the date, followed by the cast (C) replicate number and the depth (D; $1=2$ meters, $2=3$ meters, $3=4$ meters).

Table S3. The taxonomic composition of the co-occurring protist cohorts generated by the kmedoids clustering analysis.

*Supplementary tables available upon request 


\section{CHAPTER THREE}

Modeling of Group II Syndiniales infections using Bayesian inference estimates nutrient flux associated with infections in a shallow coastal pond

Taylor R. Sehein ${ }^{1}$, Gregory L Britten², Jeremy Szymczak ${ }^{3}$, Mick Follows², Laure Guillou ${ }^{3}$, Rebecca Gast ${ }^{4}$, Virginia P. Edgcomb ${ }^{5}$

${ }^{1}$ MIT-WHOI Joint Program in Biological Oceanography, Cambridge and Woods Hole, MA, United States

${ }^{2}$ Department of Earth, Atmosphere, and Planetary Science, Massachusetts Institute of Technology, Cambridge, MA, United States

${ }^{3}$ Sorbonne Université \& Centre National pour la Recherche Scientifique, Station Biologique de Roscoff, Roscoff, France

${ }^{4}$ Department of Biology, Woods Hole Oceanographic Institution, Woods Hole, MA, United States

${ }^{5}$ Department of Geology and Geophysics, Woods Hole Oceanographic Institution, Woods Hole, MA, United States

\section{STATEMENT OF CONTRIBUTION}

Cultures were maintained by the Roscoff culture collection with infection experiments performed by Jeremy Szymczak under the guidance of Laure Guillou. Cell counts for these experiments were performed by Taylor Sehein and Rebecca Gast. Samples were collected and processed from Salt Pond by TS, RG, Maria Pachiadaki, and Virginia Edgcomb. TS performed all environmental cell counts. Model design was overseen by Mick Follows. Bayesian analyses were performed by Gregory L. Britten. TS, GLB, and MF analyzed the model performance. TS wrote the paper with input from GLB and comments from the other co-authors. 


\begin{abstract}
The order Syndiniales (phylum Dinoflagellata) represents one of the most hyperdiverse lineages recovered in global ocean microeukaryote marker gene surveys; however, only a fraction of this diversity has been cultivated. Formally described Syndiniales are obligate intracellular parasites of protists and metazoa. Termination of infections results in host death and cell lysis, which releases dissolved and particulate matter into the surrounding water column. Observations of infection events in marine systems are limited by sampling frequency, and quantifications of total impacts on host populations and nutrient fluxes are complicated by spatial heterogeneity (between depths and areal extent) of host and parasite populations. In this study, laboratory and field observations of syndinian infections were used to inform models of host-parasite interactions based on equations first proposed by Salomon and Stolte (2010). A Bayesian approach was used to fit the model equations to observed concentrations of hosts and parasites in culture and in coastal waters. The distributions of estimated parameter values characterizing host and parasite dynamics showed potential interspecies variation. For interactions resulting in the collapse of the host population, dissolved nutrients were measured at the start and termination of the culture experiment. Infections were inferred over time and integrated to estimate the total infections per culture experiment and field infection event. For the culture-based experiment, the concentrations of dissolved nutrients were divided by the total infections to estimate the dissolved organic carbon, ammonium, nitrate and nitrite released from an individual infected cell. The per cell quantities were then used to calculate first estimates of select nutrients released per syndinian infection event observed in a coastal pond.
\end{abstract}

\title{
INTRODUCTION
}

Protists belonging to the order Syndiniales are ubiquitous in marine microbial communities. This taxonomically diverse lineage is represented by over 100 clades defined by phylogenetic analyses (Guillou et al., 2008, Cai et al., 2020); however, limited ecological information is available to differentiate the niches of individual clades. All strains observed to date have exhibited a parasitic lifestyle that results in the death of the host at the completion of the life cycle (Coats and Park, 2002, Guillou et al., 2008, Chambouvet et al., 2008, Chambouvet et al., 2011, Siano et al., 2011). In microeukaryotes, infections are initiated when a free-living spore attaches to a host cell and invades the nucleus or cytoplasm. The parasite develops into a mature trophont within the host and metamorphoses via sporogenesis, producing dozens to hundreds of 
new bi-flagellated spores that are released into the surrounding water column (Coats and Park, 2002). Syndinian infections have the potential to influence the succession of plankton populations by targeting specific hosts (Cai et al., 2020) and impact local nutrient concentrations through the release of dissolved and particulate organic matter from host cell lysis (Chambouvet et al., 2008, Siano et al., 2011, Velo-Suarez et al., 2013). Therefore, elucidating the extent of infections by Syndiniales in different marine systems is essential for estimating their influences on microbial community structure and on releases of nutrients into the water column.

Current paradigms of marine carbon cycling (e.g., Worden et al. 2015) now recognize and include parasites, protist grazing, and viral lysis in depictions of transfers of carbon between the euphotic zone, aphotic zone, and the benthos, however little quantitative information exists on the impact of marine protist parasites. In contrast, there have been many studies of the impacts of protist grazing (Sherr and Sherr, 2002, 2018, Rocke et al., 2015, Pachiadaki et al., 2016, Orsi et al., 2018, Hu et al., 2021), and viruses have been studied in a number of marine and aquatic environments, with estimates of carbon released from viral lysis ranging from $0.1-0.6 \mu \mathrm{g}$ L-1 d-1 (offshore Gulf of Mexico; Proctor and Fuhrman, 1991) to $8.08 \mu \mathrm{g} \mathrm{L}-1 \mathrm{~d}-1$ in the anoxic hypolimnion of Plufisee, a eutrophic lake in northern Germany (Weinbauer and Hofle, 1998). Similar to the viral shunt, host cell lysis from parasitic infections releases labile forms of carbon and other nutrients back into the water column where they can be used to fuel microbial metabolic processes (Yih and Coats, 2000, Salomon et al., 2009, Skovgaard, 2014). Unfortunately, individual sources of dissolved and particulate nutrients cannot be easily traced in the marine environment, making it difficult to quantify the specific impacts of parasitism on biogeochemical cycling. Furthermore, temporal variation in sources and sinks of nutrients in open-ocean systems also complicates quantification of the release of nutrients from any specific process. Widespread and abundant molecular signatures of marine Syndiniales parasites (e.g. Lopez-Garcia et al., 2001, Edgcomb et al., 2011, Pernice et al., 2015, de Vargas et al., 2015, Clarke et al., 2019, Anderson and Harvey, 2020) suggest they may exert significant top-down pressures on protist populations throughout the global ocean; however, no quantitative data exist to describe the particulate and dissolved organic matter released by infection events.

Known host phyla of Syndiniales parasites include dinoflagellates, ciliates, radiolarians, copepods, and other crustacea (Cachon and Cachon, 1970, Coats et al., 1996, Stentiford and Shields, 2005, Guillou et al., 2008, Bachvaroff et al., 2012, Coats et al., 2012, Brate et al., 2012); 
however, the host ranges of most individual clades remains undefined. Studies of some established cultures of syndinian parasites indicate some strains are generalists that infect multiple hosts, while others are specialized to infect specific taxa (Cai et al., 2020). Variations in these strategies can occur at the sub-clade level, suggesting cryptic diversity that may have ecological implications. Current methods of identifying infections in environmental samples, including fluorescence in-situ hybridization and epifluorescent microscopy (Chambouvet et al., 2008), are insufficient alone for characterizing specific host-parasite interactions at the strain level. Isolation and culturing of syndinian parasites has been challenging, and has been successful for representatives of only a few clades because it requires establishing co-cultures with compatible hosts.

High spatial and temporal sampling of marine communities can be used to detect the presence of parasite populations and infection rates among susceptible hosts. However, such studies are time- and labor-intensive and often not practical when sites of interest are far from shore. Models of host-parasite systems, on the other hand, can simulate infection dynamics and can be used to estimate the total impacts of these interactions when high-resolution spatial and temporal datasets are not readily available. Equations for parasitic interactions, first proposed by Anderson and May (May, 1978, Anderson and May, 1980, 1981), were based on elements of epidemiology and predator-prey systems. Lotka-Volterra formulations assume mortality of the prey, which is comparable to the lysis of host cells from syndinian infections (Coats and Bockstahler, 1994, Coats and Park, 2002, Kim et al., 2004). Based on these assumptions, Salomon and Stolte (2010) proposed equations to characterize the interactions of syndinian parasites with three dinoflagellate hosts based on the Lotka-Volterra predation model with a modified Rosenzweig-MacArthur model (Rosenzweig, 1971). This seminal work identified three "state" variables (hosts, free-living parasites, and infections in-progress) that best reflected the natural behavior of host-parasite systems. Differences in measured parameter values, such as host growth rate, parasitoid encounter rate, spore production, and parasite mortality, influenced variation in temporal dynamics for individual dinoflagellate-parasite systems (Salomon and Stolte, 2010). The equations proposed by Salomon and Stolte greatly simplified host-parasite systems by excluding other possible mortality factors such as grazing by other protists and Metazoa, hydrodynamic processes, and alternative life stages (such as dormancy; Chambouvet et 
al., 2011b); however, it provided an easily interpretable framework for examining host-parasite interactions in culture and for making comparisons to parasitic infections in the field.

Few studies of Syndiniales in marine systems have generated sufficient data sets for testing the parasitism model proposed by Salomon and Stolte. Here, we leverage Bayesian inference to evaluate the Salomon and Stolte model using culture-based and field observations from a high-resolution time series performed in a shallow coastal pond (Salt Pond, Falmouth, MA, USA) during the summer of 2018 (Sehein et al., in review). Two culture-based experiments were used to assess the model's ability to capture infection dynamics between two small coastal dinoflagellate species, Heterocapsa triquetra and Scrippsiella acuminata, and a generalist Group II-Clade 2 Syndiniales parasite capable of infecting both taxa (A120). The model was fit to three infection events observed in Salt Pond at nearly monthly intervals in July, August, and September 2018. The boom-bust infection dynamics recorded in Salt Pond are consistent with other observations of host-parasite interactions, including those used to parameterize the Salomon and Stolte model (Coats and Park, 2002) and studies of syndinian infections in marine systems (Chambouvet et al., 2008, Siano et al., 2011, Velo-Suarez et al., 2013). If the model of host-parasite interactions accurately describes the observations in Salt Pond, we hypothesize that parameter values for each infection event can be estimated, providing an overall characterization of the mixed host and parasite populations for which direct measurements are not possible. Furthermore, nutrient analyses from the culture-based experiments and estimates of total parasitized cells generated by tracking the state variables over time provided per cell estimates of carbon and nitrogen released during host cell lysis that were used to make estimates of nutrient flux in the environment.

\section{METHODS}

Host-parasite infection experiments and nutrient analyses sample preparation

Cultures of two host and parasite strains were isolated and maintained by the Roscoff Culture Collection. Host strains (Scrippsiella acuminata, ST147, and Heterocapsa triquetra, HT150) were grown in batch cultures. The parasite strain, A120, was maintained in separate batch cocultures with each host (ST147 and HT150) prior to inoculation at the start of the experiment. At the start of each experiment, 1.07L of host culture (ST147: $5.5 \times 10^{6}$ cells/L, HT150: 1.1 $10^{7}$ cells/L) was mixed with $535 \mathrm{~mL}$ of dinospores filtered from the corresponding parasite co-culture 
(ST147-A120: $6.7 \times 10^{8}$ cells/L, HT150-A120: $1.8 \times 10^{8}$ cells/L), resulting in an initial parasite:host ratio of 120:1 for the Scrippsiella acuminata:A120 culture and 16:1 for the Heterocapsa triquetra:A120 culture. Aliquots of $125 \mathrm{~mL}$ were transferred to $15200 \mathrm{~mL}$ ventilated culture flasks and incubated for 12 hours in a culture cabinet set at $20^{\circ} \mathrm{C}$ with a light intensity of $100 \mathrm{uE}$.

After 12 hours, bacteria and any free-living parasite spores that had not initiated an infection in a host cell were removed by filtering the individual culture flasks and resuspending cells in new media that included an antibiotic mixture. First, host cells were filtered through

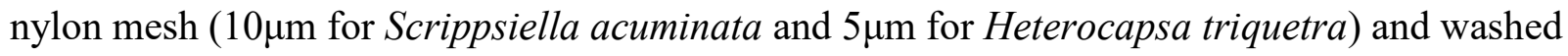
into a $50 \mathrm{~mL}$ Falcon tube with $30 \mathrm{~mL}$ of new media. This step was repeated for all individual culture flasks. Cells suspended in the new culture medium were then transferred to a large flask and $83 \mathrm{~mL}$ of a penicillin-streptomycin-neomycin antibiotic mixture (final concentration of $2500 \mathrm{U}$ penicillin, $2.5 \mu \mathrm{g} / \mathrm{mL}$ streptomycin, $5 \mu \mathrm{g} / \mathrm{mL}$ neomycin) was introduced to the culture. Bacterial concentrations were monitored throughout the experiments, ensuring growth of nontarget prokaryotes was minimal. The medium was adjusted to a final volume of $1.66 \mathrm{~L}$ and $110 \mathrm{~mL}$ aliquots of culture were placed into 15 new $200 \mathrm{~mL}$ ventilated culture flasks and incubated in a culture cabinet at $20^{\circ} \mathrm{C}$. Three flasks each were removed at the time points 0,16 , 25, 49 and 86 hours for the Scrippsiella acuminata-A120 (Syndiniales Group II Clade 2) culture and $0,25,49,67.5$, and 86 hours for the Heterocapsa triquetra-A120 culture. When three flasks were chosen at random for each time point, the remaining cultures were rotated in the culture cabinet to reduce the influence of any light variability.

At each time point, samples for catalyzed reporter deposition fluorescence in-situ hybridization (CARD-FISH) and nutrient analyses were collected. The cultures were first mixed gently to homogenize the cells and media. Cells were preserved by removing $5 \mathrm{~mL}$ aliquots from individual culture flasks and adding $500 \mu \mathrm{L}$ of paraformaldehyde (4\% final concentration) to each sample in a Falcon tube. Samples were incubated for 1-3 hours at $3^{\circ} \mathrm{C} .5 \mathrm{~mL}$ of preserved sample were filtered through $25 \mathrm{~mm} 0.2 \mu \mathrm{m}$ Millipore Isopore filters using gentle vacuum pressure and rinsed using a series of ethanol washes $(5 \mathrm{~mL}$ of chilled $50 \%, 80 \%$ and $100 \%$ ethanol, incubated for 3 minutes each at room temperature). Filters were allowed to dry, then stored at $-20^{\circ} \mathrm{C}$. Timepoint nutrient analyses were processed for ammonium, nitrate + nitrite, and dissolved organic carbon (DOC) analyses after filtering culture media through a sterile $0.2 \mu \mathrm{m}$ Sterivex filter using 
a sterile syringe. $15 \mathrm{~mL}$ of filtered media was collected in sterile HDPE Nalgene bottles and stored at $-20^{\circ} \mathrm{C}$ for nitrogen anion analyses. Samples were measured on an AQ2 Discrete Analyzer at the Florida Atlantic University Water Analysis Lab. 40mL of filtered media were collected in glass septa-capped vials with no headspace and stored at $4^{\circ} \mathrm{C}$ for dissolved carbon analyses. $40 \mathrm{~mL}$ of unfiltered media was collected in a separate glass septa-capped vials with no headspace and stored at $4^{\circ} \mathrm{C}$ for total carbon analysis. Carbon analyses were performed at the Florida Atlantic University Water Analysis Lab on a Shimadzu TOC-L system with Total Nitrogen module using the non-purgeable organic carbon method.

\section{Field collections}

Seawater samples were collected from a shallow coastal pond (maximum depth 5.5 meters; Salt Pond, Falmouth, MA, USA) every two to three days from March to October 2018. A handheld YSI (YSI Pro2030, Ohio, USA) was used to record temperature, salinity, and dissolved oxygen profiles at the sampling site located at the deepest point in the pond $(5.5 \mathrm{~m})$. Two replicate casts were performed at two meters depth using a 1.7L GoFlo bottle (General Oceanics, Florida, USA). From each cast, $600 \mathrm{~mL}$ of seawater was preserved with a final concentration of $4 \%$ formalin and stored on ice for transport to the laboratory. Environmental measurements from each sampling date are described in detail in Sehein et al. (in review, Table S1). Free-living parasite spores and infected host cells were collected on filters for CARD-FISH. To enumerate free-living spores, $20 \mathrm{~mL}$ of formalin-fixed seawater from each cast was filtered on a black $47 \mathrm{~mm} 0.2 \mu \mathrm{m}$ Millipore Isopore filter and washed three times with sterile $5 \mathrm{~mL} 1 \mathrm{x}$ phosphate buffered saline (PBS). Infected hosts were concentrated on white $47 \mathrm{~mm} 3.0 \mu \mathrm{m}$ Millipore Isopore filters and washed three times with $5 \mathrm{~mL} 1 \mathrm{x}$ PBS. All filters were stored at $-20^{\circ} \mathrm{C}$.

\section{Enumeration of infections and free-living spores using CARD-FISH}

Filters for enumerating infected hosts and free-living spores were hybridized using the horseradish peroxidase oligonucleotide probe, ALV01, that targets 75 of 125 clades of Group II Syndiniales. The parasite strain used for the culture experiments was compatible with the ALV01 probe; however, we acknowledge that use of this probe may have missed some strains of Group II parasites present in Salt Pond, resulting in an undercount of infections and free-living spores in our environmental samples. The hybridization protocol is described in Sehein et al. (in review) 
and detailed at protocols.io (dx.doi.org/10.17504/protocols.io.bsxmnfk6). To enumerate freeliving spores, $20 \mathrm{~mL}$ of each Salt Pond water sample was filtered onto $0.22 \mu \mathrm{m}$ Millipore Isopore $47 \mathrm{~mm}$ filters. $200 \mathrm{~mL}$ of each water sample was filtered onto $3.0 \mu \mathrm{m}$ Millipore Isopore $47 \mathrm{~mm}$ filters to enumerate infected hosts. Hybridized cells were visualized on an Axio Imager M2 epifluorescence microscope at 400x magnification using the FITC filter set to visualize the fluorescein-labeled ALV01 probe. The DAPI filter set was used to view the presence or absence of host theca stained with Calcofluor White, and the Cy3 filter set was used to confirm the presence of nuclei stained with propidium iodide in all putative parasite cells. To enumerate freeliving parasite spores hybridized with the ALV01 probe, spore cells were visualized at 400x magnification. All spores were counted on $1 / 8$ filter wedges when spores were low-density (less than 1,000 spores per filter wedge). Higher density spore concentrations were estimated by enumerating labeled cells in the grid of the ocular micrometer of the microscope eyepiece until either 400 spores or 40 grids were counted. A "cells per grid" value was calculated and multiplied by the total number of grids on $1 / 8$ of the filter, which was calibrated by comparing the ocular grid to a stage micrometer slide. Infected hosts were enumerated by counting all cells labeled with the ALV01 probe on $1 / 8$ of the filter. Uninfected hosts were estimated by using the same technique used for enumerating high concentrations of free-living spores.

\section{Bayesian modeling of infection dynamics}

Modified equations from Salomon and Stolte (2010) were fit to the observed counts of host cells, infected cells, and spores. The equations are:

$$
\begin{aligned}
& \text { 1. } \frac{d H}{d t}= r H\left(1-\frac{H}{K}\right)-\left(\frac{a H}{1+a h_{1} H}\right) P \\
& \text { 2. } \frac{d I}{d t}=\left(\frac{a H}{1+a h_{1} H}\right) P-\frac{I}{h_{2}} \\
& \text { 3. } \frac{d P}{d t}=\frac{e I}{h_{2}}-m P
\end{aligned}
$$

where state variables $\mathrm{H}$, I, and P are host counts, infected cell counts, and spore counts, respectively, $\mathrm{r}$ is the maximum growth rate of the host (per day), $\mathrm{K}$ is the carrying capacity of the host (cells/L), a is the parasitoid attack rate (liters per dinospore per day), $\mathrm{h}_{1}$ is the handling time (days), $\mathrm{h}_{2}$ is the turnover timescale of the infected cells (days), $\mathrm{e}$ is the reproductive output (spores per infection), and $\mathrm{m}$ is the mortality rate (per day). Where Salomon and Stolte (2010) had $h_{1}=h_{2}$, we have the more general case $h_{1} \neq h_{2}$. We estimate the parameters of the model via 
Bayesian inference using Markov Chain Monte Carlo (MCMC) sampling. A priori parameter distributions were calculated from values observed by Coats and Park (2002) for the hosts Akashiwo sanguinea, Levanderina fissa (formerly Gymnodinium instriatum), and Karlodinium micrum and corresponding parasite strains. We used normal prior distributions for each parameter with means and standard deviations given by the means and standard deviations of the Coats and Park (2002) estimates, respectively. The inference was implemented in the Stan programming language (Carpenter et al. 2017) and called from the R package rstan (Stan Development Team 2020). Four MCMC chains were run with 2000 iterations each. The GelmanRubin R statistic was monitored to ensure $\mathrm{R}<1.1$, indicating MCMC convergence (Gelman and Rubin 1992). The carrying capacity parameters were fixed at $10^{7}$ (above the largest observed host count) and were not included in the inference due to poor estimability in the model. Sensitivity analyses showed that variations in the assumed carrying capacity did not impact the results, except when set at unreasonably low values (Figure S1). Our statistical approach extends Salomon and Stolte (2010) by constraining the underlying parameter distributions using the host cell, infected cell, and spore time series. The Bayesian aspect allows us to combine parameter information from Coats and Park (2002) with new observations and formally quantify underlying uncertainties. Stan and R code to fit the model and make the figures is available at https://github.com/gregbritten/host parasite_public.

\section{Calculation of nutrient flux}

Nutrient samples were collected from the Scrippsiella acuminata-A120 co-culture at T12 (after removal of free-living spores and addition of antibiotics) and at T96 when most infections had terminated, lysing the host cells and releasing free-living parasite spores. The change in concentration of DOC, ammonium, and nitrate and nitrite were calculated between these two timepoints. The change in nutrient concentrations between T12 and T96 represented the transformation in nutrient pools from the initiation to the termination of an infection event. The modeled rates of infection were examined for the host-parasite experiments and field infection events where the model fit closely agreed with the observed concentrations of host infections and free-living parasites. We time-integrated the term $\mathrm{I} / \mathrm{h}$ over the course of the events which described the number of cells that had completed the infection cycle. Each of the nutrient concentration changes $(\mu \mathrm{g} / \mathrm{L})$, calculated as described above, were divided by the total number of 
infected S. acuminata cells estimated by the model (infections/L), generating a "per cell" nutrient flux value for cells of a similar size to Scrippsiella acuminata ( $\mu \mathrm{g} / \mathrm{infection})$. Although all infected cells in an environmental sample were likely not the same size and nutrient composition as the culture of $S$. acuminata, the per cell nutrient concentrations were used as a rough estimate of the nutrient flux resulting from all observed infected cells during each infection event in Salt Pond.

\section{RESULTS}

\section{Model performance with host-parasite co-cultures}

Concentrations of uninfected hosts, infected hosts, and free-living parasite spores observed across the five experimental time points (red markers) were compared to the modeled dynamics. The means for the state variables (across the MCMC iterations) and the $95 \%$ credible intervals were calculated and are displayed as solid black and dashed lines, respectively, in Figure 1.

\section{Modeling of field observations}

Observations of infected hosts and free-living parasite spores in Salt Pond were used to test the model's applicability to host-parasite systems outside of the laboratory. Data corresponding to three infection events that occurred in July (Event 1), August (Event 2), and September (Event 3 ), were separated into individual model runs. A priori parameter distributions were the same $a$ priori distributions as derived from Coats and Park (2002) in all model runs. Unlike the culturebased experiments, concentrations of uninfected host cells could not be directly measured because samples contained an assemblage of susceptible and non-susceptible protistan taxa that could not be distinguished morphologically. Uninfected host cell concentrations were therefore inferred as an unobserved variable by the model based on the concentrations on infected hosts and free-living spores. The observed concentrations of infected hosts and free-living parasite spores (red markers) are presented in Figure 2 along with the model-generated mean (solid black) and $95 \%$ credible interval (dashed lines).

\section{Comparison of parameter distributions}

The values of the model parameters $r$ (host growth rate; per day), $h_{2}$ (infection turnover time; days), $m$ (parasite mortality rate; per day), and $e$ (parasite reproductive output; spores per 
infection) were plotted for infection events for which model fits indicated higher degrees of certainty (described by narrower $95 \%$ credible intervals) and in-situ observations closely matched model fits. The parameters $a$ (parasitoid search rate; per day) and $h_{1}$ (infection initiation time; days) were plotted together in the combined term a/ $\left(1+a_{1}\right)$ to represent the initiation of new infections. The solid lines represent the mean parameter values, with $95 \%$ credible intervals represented by the dashed lines. Narrower distributions of parameter values indicate higher degrees of certainty for the given values. The overlap between parameter distributions indicates the degree of similarity for a given parameter.

\section{Estimates of nutrient flux from syndinian infections}

The total infected cells integrated over time are reported in Table 1 for the S. acuminata-A120 culture and the Salt Pond infection events 1 and 3. Total infected cells for the H. triquetra-A120 culture and event 2 in Salt Pond could not be accurately calculated due to poor model fit. Estimates of the dissolved carbon and nitrogen concentrations per cell assumed limited host cell growth and nutrient uptake because host cells do not appear to divide when infected by syndinian parasites and infections were pervasive in the S. acuminata-A120 cultures. The per cell estimate of dissolved organic carbon released was $31.66 \mathrm{ng} /$ cell, which equaled $1130 \mu \mathrm{gC} / \mathrm{L}$ (Event 1) and $920 \mu \mathrm{gC} / \mathrm{L}$ (Event 3) released per infection event in Salt Pond. The concentrations of ammonium $(0.14 \mathrm{ng} / \mathrm{cell})$ and nitrate plus nitrate $(0.05 \mathrm{ng} / \mathrm{cell})$ resulted in estimates of $4.4 \mathrm{ug} / \mathrm{L}$ and $3.6 \mathrm{ug} / \mathrm{L}$ ammonium and $1.6 \mathrm{ug} / \mathrm{L}$ and $1.3 \mathrm{ug} / \mathrm{L}$ nitrate plus nitrite released in Salt Pond in total for events 1 and 3, respectively.

\section{DISCUSSION}

Marker gene signatures of marine parasites are ubiquitous in samples collected across the global ocean (Pernice et al., 2015, de Vargas et al., 2015); however, overarching questions remain regarding the degree to which parasitism both impacts biogeochemical cycling and exerts topdown controls on host populations. It can be challenging to quantify host infections in aquatic ecosystems and to trace the sources and sinks of nutrients resulting from host cell lysis due to parasitism. Here, data from culture-based experiments and field observations were used to inform a model using Bayesian approaches and gain new insights into the impacts of syndinian 
parasitism on protistan hosts and to make estimates of nutrients released from the termination of an infection cycle. The non-linear equations for host-parasite infection dynamics proposed by Salomon and Stolte (2010) provided the initial framework for modeling interactions between two hosts, Heterocapsa triquetra and Scrippsiella acuminata, and the Syndiniales Group II Clade 2 parasite strain A120. The selected dinoflagellates are common coastal species that have been found to host various clades of Group II Syndiniales parasites across geographically-distinct environments (Coats et al., 1996, Gunderson et al., 2002, Kim, 2006, Chambouvet et al., 2008, Chambouvet et al., 2011, Li et al., 2014). Co-cultures of each host with the parasite were introduced to new cultures of each host and infections were monitored at approximately $24 \mathrm{hr}$. intervals over four days. Three replicates were removed at each timepoint to quantify cell concentrations and nutrients. Bayesian approaches were then used to fit the modified Salomon and Stolte model equations to observations of uninfected hosts, infected hosts, and free-living spores over the course of the culture-based experiments.

Parasite virulence differed between the two host populations. Infections in H. triquetra reached $13.66 \%$ of cells in the culture at 25 hours and declined as a percentage of host cells thereafter. The reduction in top-down pressure from the parasite and optimal growth conditions for the hosts likely enhanced the proliferation of the host population (Figure 1). Infections in $S$. acuminata peaked at 15 hours, impacting $61.12 \%$ of the population, with greater than $50 \%$ of remaining cells infected at the termination of the experiment after 96 hours. This translated to a ten-fold decline in the original host cell concentration. In the model framework proposed by Salomon and Stolte, the stable coexistence of host-parasite systems is modulated by the parameter K (carrying capacity in cells/L) when all other parameter values remain fixed (Salomon and Stolte, 2010 [Figure 3]). Modifications to the value K simulate non-stable host and parasite outcomes, such as those observed in the culture experiments. K values might also be adjusted to reflect environmental growth limitations when the nutrient concentrations and the host cell content are known. For example, the carbon content of a $S$. acuminata cell from the ST147 culture was estimated to be 2.27 pg C (Fayal et al., 2020) and the total organic carbon measured in the culture media was $7 \mathrm{mg} \mathrm{C} / \mathrm{L}$; therefore, approximately $10^{8}$ could theoretically exist in culture if carbon was the limiting nutrient. In Salomon and Stolte (2010), a model outcome where the host population persists and parasite population declines is labeled Type I and is associated with lower carrying capacities. $\mathrm{K}$ values were tested across eight orders of 
magnitude in an effort to replicate the Type I behavior observed in the H. triquetra-A120 culture; however, no K values significantly improved the model fit and it could not be inferred reliably by the model (Figure S1). On the other hand, the S. acuminata and A120 populations exhibited boom-bust population dynamics similar to Lotka-Volterra predation models. This pattern is described by Salomon and Stolte as Type III and is associated with higher K values. With K set to $10^{7}$ cells/L, a model fit was generated that closely replicated the culture-based observations. This suggests the equations within the existing model framework better reflect Type III infection behaviors and additional parameters may need to be considered in order to replicate other population dynamics.

The original Salomon and Stolte model equations and parameter values obtained from experimental data (Coats and Park, 2002) were compared to field data obtained in the Penzé estuary by Chambouvet et al. (2008), who reported in-situ dinospore concentrations that were much lower than predicted by the model. The parameter values used in the model were based on measurements from host-parasite cultures; however, parameter values may differ for protist populations interacting in the marine environment. We reexamined the Salomon and Stolte model using a Bayesian approach, which estimates parameter distributions rather than assigns fixed parameter values, and fit the model using observations of infection events documented in a high-resolution time series collected from Salt Pond (Falmouth, MA, USA) in 2018. In Salt Pond, between March and October, three infection events (July, August, and September) were documented by enumerating infected hosts and numbers of free-living parasite spores using fluorescence in-situ hybridization (FISH). iTag barcode libraries characterized parasite diversity during each of these infection events and, combined with microscopy data, informed on putative host identities (Sehein et al., in review). Group II-Clade 2 Syndiniales parasites represented over $95 \%$ of all parasite sequences on July $3^{\text {rd }}$ (Event 1) and microscopy identified scrippsielloid and heterocapsid host morphotypes. Event 2 infections reached a maximum on August $13^{\text {th }}$ with diverse parasite clades represented in iTag libraries at that time point, and multiple thecate and athecate dinoflagellate host morphologies present on FISH filters. Parasite infections during Event 3 peaked on September $14^{\text {th }}$, when $97 \%$ of parasite sequence reads were identified as Group II-Clade 30, and documented hosts only included one unidentified athecate dinoflagellate morphotype. Because these infection events each involved unique combinations of hosts and parasites, each event was modeled individually (Figure 2). Uninfected host concentrations were 
synthetically derived by the model because pools of host populations represented only a fraction of the overall protist community, and because not all individual host taxa could be accurately identified and enumerated based on morphological features on FISH filters. Similar to the outcome of the two laboratory culture-based studies, infection events that followed the Type III boom-bust cycle (Events 1 and 3) could be modeled by the Salomon and Stolte equations. Event 2 , on the other hand, exhibited very low dinospore concentrations throughout the infection event. It was hypothesized in Sehein et al. (in review) that increased grazing pressure from ciliates (Orsi et al., 2018) or other hydrodynamic processes may have more rapidly removed free-living spores from the system during this event. The a priori distributions for the parasite mortality term, $m$, were widened and the model was refit for Event 2; however, this did not improve the model outcome. Additional grazing terms may be needed to incorporate food web interactions that impact parasite spore production.

Unlike the fixed parameter values measured by Coats and Park (2002), Bayesian approaches produce a range of values that are probable based on observations (i.e. infected host and free-living spore concentrations). Parameter values for neither the S. acuminata-A120 laboratory experiment nor the field observations (Events 1 and 3) could be directly measured or calculated using the same methodologies described by Coats and Park (2002). However, modeling these interactions with Bayesian inference produced parameter distributions that allowed for comparison between the laboratory and field infection dynamics in this study (Figure 3 ) and previous experiments. For example, host growth rates $(r)$ differed between the laboratorygrown S. acuminata cells $\left(0.39 \mathrm{~d}^{-1}\right)$ and the host populations in Salt Pond (mean values of $0.18 \mathrm{~d}^{-}$ ${ }^{1}$ and $0.085 \mathrm{~d}^{-1}$ for events 1 and 3 , respectively). The estimated growth rate of the S. acuminata culture was close to the growth rate of the similarly-sized Karlodinium micrum calculated by Coats and Park (2002, $\left.0.35 \mathrm{~d}^{-1}\right)$, which aligns with previously reported relationships between cell size and growth rate in culture (Reynolds, 1984, Chisholm, 1992, Tang 1995). Growth rates in marine systems, however, can differ from culture-based observations due to variations in nutrient availability, light intensity, and other environmental factors (Irigoien et al., 2005). In contrast to previous applications of the Salomon and Stolte model where parameters were fixed, our Bayesian inference procedure estimated the parameters that are most consistent with the observed dynamics and prior knowledge of the parameters, thus improving the model fit and 
yielding parameters that better reflect the characteristics of the taxa in the marine environment that was sampled.

We modified the parameter for handling time, $h$, and assumed the duration was not equal for the different terms describing the initiation and termination of an infection. In practice, this distinction is not easily observed because the progression of an infection is continuous, but cells are observed at fixed time points. The parameter $h_{l}$ was used in the term $(\mathrm{aH} /(1+\mathrm{ah} 1 \mathrm{H})) \mathrm{P}$ seen in equations 1 and 2. Here, the handling time is describing the transformation of uninfected hosts to infected hosts. $h_{2}$, found in the term $\mathrm{I} / \mathrm{h} 2$ in equations 2 and 3 , describes the turnover time when the parasite infection lyses the host cell and the spores are released. When the model was fitted to the S. acuminata-A120 and Salt Pond observations, the $h_{1}$ and $h_{2}$ parameter distributions differed (Figure S2) suggesting the time to generate a new infection is not equal to the turnover time. This modification to the parameter term $h$ may improve model fits by better-characterizing infection dynamics that are not easily quantified.

The infection "initiation term," $\left(a / 1+a h_{1}\right)$, is represented by both the parasitoid search rate, $a$, and the initial handling time, $h_{1}$, with larger values indicating more host cells are transitioning from an uninfected to infected state. Values for this term were greater for the Salt Pond infection events than for the S. acuminata-A120 culture, which suggests parasite spores are more efficient in locating host cells and initiating new infections, even in more dilute environments. The initiation of new infections may be enhanced by cellular communication strategies that differ in native host and parasite populations; however, this research area remains largely unexplored for eukaryotic taxa. It could be interesting to investigate the role of signaling molecules in host-parasite interactions in future studies. The handling time of an active infection, $h_{2}$, was conserved between observations in the laboratory and Salt Pond. This is supported by parasite generation times (summarized as $h_{1}+h_{2}$ ) reported from 53-71 hours in other culturebased studies (Coats and Park, 2002, Kim et al., 2004).

Parasite mortality $(\mathrm{m})$ in the laboratory is regulated by the duration of time a spore can live outside of a host; however, many other factors, including top-down pressure from grazing, can impact spore mortality in marine systems. The mortality rate of A120 $\left(0.85 \mathrm{~d}^{-1}\right)$ was similar to the parasite strain infecting Levanderina fissa (Coats and Park, 2002) suggesting similar 
limitations of survival for these two strains in culture. Conversely, the mortality rates were twice as high $\left(2.2 \mathrm{~d}^{-1}\right)$ for the presumed Group II-Clade 2 (Event 1) and Group II-Clade 30 (Event 3) parasites in Salt Pond when compared to the A120 parasite strain infecting S. acuminata. Differences in spore production support the hypothesis that extant biological factors impact the enumerated concentrations of free-living spores, resulting in different parameter value estimates. The mean estimated spore production for infections in the S.acuminata-A120 culture is 360 spores/cell. Event 1, which included both infected scrippsielloid cells and other host morphotypes, and Event 3 both estimated approximately 85 spores per host. Spore concentrations were very low during Event 2, suggesting at times top-down pressures can be significant and may impact the ability to model some infection events with the existing framework.

The model proposed by Salomon and Stolte and modified in this study can predict the outcome of Type III infection events for which the host populations, and subsequently the parasite populations, collapse. Tracing the sources and sinks of carbon and nitrogen species released from these infection events, on the other hand, is more challenging. Cultures of $S$. acuminata and the parasite strain A120 maintained in antibiotic-treated media were used to estimate "per cell" quantities of carbon and nitrogen released through host cell lysis. Dissolved organic carbon released per cell was estimated to be $31.66 \mathrm{ng} / \mathrm{cell}$. In previous studies of Scrippsiella grown in culture, per cell carbon content was estimated to be $1.6 \mathrm{ng} / \mathrm{cell}$ in vegetative cells and $10.6 \mathrm{ng} / \mathrm{cell}$ in dormant cysts (Lirdwitayaprasit et al., 1990). When gene transcripts of another dinoflagellate, Alexandrium tamarense, were compared when grown in the presence and absence of syndinian parasites, researchers found significant upregulation of genes involved with energy supply, including glycolysis, fatty acid b-oxidation, the tricarboxylic acid cycle, oxidative phosphorylation, and the glyoxylate cycle in the presence of the parasites (Lu et al., 2016, Kayal et al., 2020, Decelle et al., 2021). Genes associated with secondary metabolites and cell signaling were also upregulated. Those data suggest that Scrippsiella may modify carbon content at different stages in the life cycle and upregulation of genes involved with energy supply may occur in response to the presence of parasites. The concentration of nitrate plus nitrite and ammonium released per host cell was estimated to be $0.05 \mathrm{ng} / \mathrm{cell}$ and 0.14 ng/cell, respectively. Lirdwitayaprasit et al. (1990) reported total nitrogen content was 0.3 ng per 
Scrippsiella cell, suggesting the estimated nitrogen released through host cell lysis is within the realm of previously measured nitrogen content.

Estimates of total infected cells for Event 1 and Event 3 in Salt Pond were 31,248 and 25,587 cells/ $\mathrm{L}$, respectively, which translates to a release of $1130 \mathrm{ugC} / \mathrm{L}$ and $920 \mathrm{ugC} / \mathrm{L}$ of dissolved organic carbon, $1.6 \mathrm{ug} / \mathrm{L}$ and $1.3 \mathrm{ug} / \mathrm{L}$ of nitrate and nitrite, and $4.4 \mathrm{ug} / \mathrm{L}$ and $3.6 \mathrm{ug} / \mathrm{L}$ ammonium from these two infection events in Salt Pond, respectively (Table 1). Nutrient turnover for protistan parasites remains largely unexplored; however, marine viral literature can be used to contextualize the abovementioned results. The Southern Ocean was sampled from December 2012 to March 2013 and found viral lysis of phytoplankton contributed to a seasonal carbon flux of $2674 \mu \mathrm{gC} / \mathrm{L}$ (Biggs et al., 2021). Incubation studies of bacteria and viruses sampled from the oligotrophic Indian Ocean and coastal False Creek (Vancouver, BC) found virus-mediated host lysis enhanced ammonium concentrations at both sites (Shelford et al., 2012), suggesting important roles for these types of interactions in nitrogen cycles across diverse marine ecosystems. The Penzé estuary was surveyed in 2004, 2005, and 2006 and found concentrations of Scrippsiella cells reached 20,000 cells/L with $20 \%$ infection prevalence $(4,000$ cells/L infected; Chambouvet et al., 2008). We do not have the data to integrate the total infected cells over time; however, if the concentration of infected in the Penzé estuary were similar to Salt Pond, we could assume comparable quantities of nutrients were released into the water column. In the Nauset Marsh System (Eastham, MA, USA), the dinoflagellate Alexandrium catenella was infected by Group II Syndiniales parasites during an annual red tide bloom in 2013 (Velo-Suarez et al., 2013). A rough integration of the total infected cells from observations made every two days indicated approximately 140,000 cells/L were infected over the duration of the bloom. The larger size of A. catenella $(\sim 40 \mu \mathrm{m})$ and abundant infections suggest this hostparasite dynamic may have a greater impact on local pools of dissolved and particulate organic matter. The effects of the nutrients released likely vary by environment. For example, in coastal, semi-enclosed ponds, like Salt Pond, stratification limits the turnover of key nutrients (including nitrogen; Behrenfeld et al., 2006, Doney, 2006, Boyce et al., 2010); therefore, the nitrogen species released through host cell lysis can supply essential nutrients for metabolic processes carried out by microbes occurring in the same water column layers where Syndiniales infections take place. 
The conclusions presented in this study support the application of the equations described by Salomon and Stolte to model host-parasite infections when populations of hosts and parasites have a "boom-bust" relationship. Relationships that followed this pattern were observed in a laboratory experiment that monitored infections in the dinoflagellate host, Scrippsiella acuminata, as well as in two infection events reported in a coastal pond. By quantifying carbon and nitrogen concentrations in the cultures, we attributed the source of the dissolved nutrients to lysed host cells and generated rough estimates of nutrients released during the infection events in Salt Pond. The estimates of carbon and nitrogen released per cell assumed the host cells observed in the field were a similar size and had similar nutrient profiles to S. acuminata; however, the contributions to pools of dissolved and particulate organic matter likely differ for hosts of varying sizes, which warrants further study and quantification. Testing the model with several unique culture and field datasets also highlighted limitations of the Salomon and Stolte equations in their current framework. The culture of H. triquetra appeared to be less susceptible to infection, which resulted in the decline of the parasite and growth of the host population. Additional culture-based studies may help quantify the susceptibility of host populations and generate a parameter that can be incorporated into the model equations. Although not included in the model equations, grazing in marine systems may also play a significant role in host-parasite interactions. Quantification of grazers in field samples and estimates of grazing rates may improve the model fittings of infection dynamics similar to Event 2. Overall, these findings validate an existing model framework that is sufficient to describe some host-parasite relationships and propose areas of research that could improve the model application to a broader range of infection dynamics.

\section{ACKNOWLEDGEMENTS}

We graciously thank the scientists the Roscoff Culture Collection (Station Biologique, Roscoff, France) for collecting and maintaining the host and parasite strains used in the culture-based experiments. We thank Jeremy Szymczak for executing the culture experiments and preparing the samples for nutrient analyses and CARD-FISH. This material is based upon work supported by the National Science Foundation Biological Oceanography OCE-1851012 to RG and VE, and by the National Science Foundation Graduate Research Fellowship under Grant No. 1745302. 


\section{REFERENCES}

Anderson, R.M. and May, R.M., 1980. Infectious diseases and population cycles of forest insects. Science, 210(4470), pp.658-661.

Anderson, R.M. and May, R.M., 1981. The population dynamics of microparasites and their invertebrate hosts. Philosophical Transactions of the Royal Society of London. B, Biological Sciences, 291(1054), pp.451-524.

Anderson, S.R. and Harvey, E.L., 2020. Temporal variability and ecological interactions of parasitic marine Syndiniales in coastal protist communities. Msphere, 5(3), pp.e00209-20.

Arbones, B., Castro, C.G., Alonso-Pérez, F. and Figueiras, F.G., 2008. Phytoplankton size structure and water column metabolic balance in a coastal upwelling system: the Ría de Vigo, NW Iberia. Aquatic Microbial Ecology, 50(2), pp.169-179

Biggs, T.E., Huisman, J. and Brussaard, C.P., 2021. Viral lysis modifies seasonal phytoplankton dynamics and carbon flow in the Southern Ocean. The ISME Journal, pp.1-8.

Bråte, J., Krabberød, A. K., Dolven, J. K., Ose, R. F., Kristensen, T., Bjørklund, K. R., and Shalchian-Tabrizi, K., 2012. Radiolaria associated with large diversity of marine alveolates. Protist, 163, pp.767-777.

Bachvaroff, T.R., Kim, S., Guillou, L., Delwiche, C. F., and Coats, D.W., 2012. Molecular diversity of the syndinean genus Euduboscquella based on single-cell PCR analysis. Applied Environmental Microbiology, 78, pp.334-345.

Behrenfeld, M.J., O’Malley, R.T., Siegel, D.A., McClain, C.R., Sarmiento, J.L., Feldman, G.C. et al., 2006. Climate-driven trends in contemporary ocean productivity. Nature, 444, pp.752755.

Boyce, D.G., Lewis, M.R., and Worm, B., 2010. Global phytoplankton decline over the past century. Nature, 466, pp.591-596.

Cachon, J. and Cachon, M., 1970. Ultrastructure des Amoebophryidae (Péridiniens Duboscquodinida) II Systèmes atractophriens et microtubulaires; leur intervention dans la mitose. Protistologica, 6, pp.57-70.

Cai, R., Kayal, E., Alves-De-Souza, C., Bigeard, E., Corre, E., Jeanthon, C., Marie, D., Porcel, B.M., Siano, R., Szymczak, J. and Wolf, M., 2020. Cryptic species in the parasitic Amoebophrya species complex revealed by a polyphasic approach. Scientific Reports, 10(1), pp.1-11.

Carpenter, B., Gelman, A., Hoffman, M.D., Lee, D., Goodrich, B., Betancourt, M., Brubaker, M., Guo, J., Li, P. and Riddell, A., 2017. Stan: A probabilistic programming language. Journal of Statistical Software, 76(1), pp.1-32. 
Cermeño, P., Marañón, E., Rodríguez, J. and Fernández, E., 2005. Size dependence of coastal phytoplankton photosynthesis under vertical mixing conditions. Journal of Plankton Research, 27(5), pp.473-483.

Cermeno, P., Maranón, E., Pérez, V., Serret, P., Fernández, E. and Castro, C.G., 2006. Phytoplankton size structure and primary production in a highly dynamic coastal ecosystem (Ría de Vigo, NW-Spain): Seasonal and short-time scale variability. Estuarine, Coastal and Shelf Science, 67(1-2), pp.251-266.

Chambouvet, A., Morin, P., Marie, D., and Guillou, L., 2008. Control of toxic marine dinoflagellate blooms by serial parasitic killers. Science, 322, pp.1254-1257.

Chambouvet, A., Laabir, M., Sengco, M., Vaquer, A., and Guillou, L., 2011. Genetic diversity of Amoebophryidae (Syndiniales) during Alexandrium catenella/tamarense (Dinophyceae) blooms in the Thau lagoon (Mediterranean Sea, France). Research in Microbiology, 162, pp.959-968.

Chambouvet, A., Alves-de-Souza, C., Cueff, V., Marie, D., Karpov, S. and Guillou, L., 2011 b. Interplay between the parasite Amoebophrya sp. (Alveolata) and the cyst formation of the red tide dinoflagellate Scrippsiella trochoidea. Protist, 162, pp.637-649.

Chisholm, S.W., 1992. Phytoplankton size. In: Falkowski PG, Woodhead WD (eds) Primary productivity and biochemical cycles in the sea. Plenum Press, New York, pp 137-213

Clarke, L.J., Bestley, S., Bissett, A. and Deagle, B.E., 2019. A globally distributed Syndiniales parasite dominates the Southern Ocean micro-eukaryote community near the sea-ice edge. The ISME Journal, 13(3), pp.734-737.

Coats, D.W. and Bockstahler, K.R., 1994. Amoebophrya ceratii in Chesapeake Bay populations of Gymnodinium sanguineum. Journal of Eukaryotic Microbiology, 41, pp.586-593.

Coats, D.W., Adam, E.J., Gallegos, C.L. and Hedrick, S., 1996. Parasitism of photosynthetic dinoflagellates in a shallow subestuary of Chesapeake Bay, USA. Aquatic Microbial Ecology, 11(1), pp.1-9.

Coats, D.W. and Park M.G., 2002. Parasitism of photosynthetic dinoflagellates by three strains of Amoebophrya (Dinophyta): parasite survival, infectivity, generation time, and host specificity. Journal of Phycology, 38(3), pp.520-528.

De Vargas, C., Audic, S., Henry, N., Decelle, J., Mahé, F., Logares, R., ... \& Carmichael, M., 2015. Eukaryotic plankton diversity in the sunlit ocean. Science, 348.

Decelle, J., Kayal, E., Bigeard, E., Gallet, B., Bougoure, J., Clode, P., Schieber, N., Templin, R., Hehenberger, E., Prensier, G. and Chevalier, F., 2021. Intracellular development and impact of a eukaryotic parasite on its zombified microalgal host in the marine plankton. bioRxiv.

Doney, S.C., 2006. Plankton in a warmer world. Nature, 444, pp.695-696. 
Edgcomb, V., Orsi, W., Bunge, J., Jeon, S., Christen, R., Leslin, C., Holder, M., Taylor, G.T., Suarez, P., Varela, R., Epstein, S., 2011. Protistan microbial observatory in the Cariaco Basin, Caribbean. I. Pyrosequencing vs Sanger insights into species richness. The ISME Journal, 5, pp.1344-1356.

Finkel, Z.V., Irwin, A.J. and Schofield, O., 2004. Resource limitation alters the 3/4 size scaling of metabolic rates in phytoplankton. Marine Ecology Progress Series, 273, pp.269-279.

Gelman, A. and Rubin, D.B., 1992. Inference from iterative simulation using multiple sequences. Statistical Science, 7(4), pp.457-472

Guillou, L., Viprey, M., Chambouvet, A., Welsh, R., Kirkham, A.R., Massana, R., Scanlan, D.J., and Worden, A.Z., 2008. Widespread occurrence and genetic diversity of marine parasitoids belonging to Syndiniales (Alveolata). Environmental Microbiology, 10, pp.3349-3365.

Hu, S.K., Herrera, E.L., Smith, A.R., Pachiadaki, M.G., Edgcomb, V.P., Sylva, S.P., Chan, E.W., Seewald, J.S., German, C.R. and Huber, J.A., 2021. Protistan grazing impacts microbial communities and carbon cycling at deep-sea hydrothermal vents. bioRxiv.

Irigoien, X., Flynn, K.J. and Harris, R.P., 2005. Phytoplankton blooms: a 'loophole'in microzooplankton grazing impact? Journal of Plankton Research, 27(4), pp.313-321.

Kayal, E., Alves-De-Souza, C., Farhat, S., Velo-Suarez, L., Monjol, J., Szymczak, J., Bigeard, E., Marie, D., Noel, B., Porcel, B.M. and Corre, E., 2020. Dinoflagellate host chloroplasts and mitochondria remain functional during Amoebophrya infection. Frontiers in Microbiology, 11.

Kim, S., Gil Park, M., Yih, W. and Coats, D.W., 2004. Infection of the bloom-forming thecate dinoflagellates Alexandrium affine and Gonyaulax spinifera by two strains of Amoebophrya (Dinophyta). Journal of Phycology, 40(5), pp.815-822.

Kim, S., 2006. Patterns in host range for two strains of Amoebophrya (Dinophyta) infecting thecate dinoflagellates: Amoebophrya spp. ex Alexandrium affine and ex Gonyaulax polygramma. Journal of Phycology, 42(6), pp.1170-1173.

Li, C., Song, S., Liu, Y. and Chen, T., 2014. Occurrence of Amoebophrya spp. infection in planktonic dinoflagellates in Changjiang (Yangtze River) Estuary, China. Harmful Algae, 37, pp.117-124.

Lirdwitayaprasit, T., Okaichi, T., Montani, S., Ochi, T. and Anderson, D.M., 1990. Changes in cell chemical composition during the life cycle of Scrippsiella trochoidea (Dinophyceae). Journal of Phycology, 26(2), pp.299-306.

Lopez-Garcia, P., Rodriguez-Valera, F., Pedros-Alio, C., Moreira, D., 2001. Unexpected diversity of small eukaryotes in deep-sea Antarctic plankton. Nature, 409, pp.603-607. 
Lu, Y., Wohlrab, S., Groth, M., Glöckner, G., Guillou, L. and John, U., 2016. Transcriptomic profiling of Alexandrium fundyense during physical interaction with or exposure to chemical signals from the parasite Amoebophrya. Molecular ecology, 25(6), pp.1294-1307.

May, R.M., 1978. Host-parasitoid systems in patchy environments: a phenomenological model. The Journal of Animal Ecology, pp.833-844.

Orsi, W.D., Wilken, S., Del Campo, J., Heger, T., James, E., Richards, T.A., Keeling, P.J., Worden, A.Z. and Santoro, A.E., 2018. Identifying protist consumers of photosynthetic picoeukaryotes in the surface ocean using stable isotope probing. Environmental Microbiology, 20(2), pp.815-827.

Pachiadaki, M.G., Taylor, C., Oikonomou, A., Yakimov, M.M., Stoeck, T. and Edgcomb, V., 2016. In situ grazing experiments apply new technology to gain insights into deep-sea microbial food webs. Deep Sea Research Part II: Topical Studies in Oceanography, 129, pp.223-231.

Pernice, M.C., Forn, I., Gomes, A., Lara, E., Alonso-Sáez, L., Arrieta, J.M., del Carmen Garcia, F., Hernando-Morales, V., MacKenzie, R., Mestre, M. and Sintes, E., 2015. Global abundance of planktonic heterotrophic protists in the deep ocean. The ISME Journal, 9(3), pp.782-792.

Proctor, L.M. and Fuhrman, J.A., 1991. Roles of viral infection in organic particle flux. Marine Ecology Progress Series, pp.133-142.

Raven, J.A., 1998. The twelfth Tansley Lecture. Small is beautiful: the picophytoplankton. Functional Ecology, 12(4), pp.503-513.

Reynolds, C.S., 1984. The ecology of freshwater phytoplankton. Cambridge university press.

Rocke, E., Pachiadaki, M.G., Cobban, A., Kujawinski, E.B. and Edgcomb, V.P., 2015. Protist community grazing on prokaryotic prey in deep ocean water masses. PLoS One, 10(4), pp.e0124505.

Rosenzweig, M.L., 1971. Paradox of enrichment: destabilization of exploitation ecosystems in ecological time. Science, 171(3969), pp.385-387.

Salomon, P.S., Granéli, E., Neves, M.H. and Rodriguez, E.G. (2009) Infection by Amoebophrya spp. parasitoids of dinoflagellates in a tropical marine coastal area. Aquatic Microbial Ecology, 55(2), pp.143-153.

Salomon, P.S. and Stolte, W., 2010. Predicting the population dynamics in Amoebophrya parasitoids and their dinoflagellate hosts using a mathematical model. Marine Ecology Progress Series, 419, pp.1-10.

Shelford, E.J., Middelboe, M., Møller, E.F. and Suttle, C.A., 2012. Virus-driven nitrogen cycling enhances phytoplankton growth. Aquatic microbial ecology, 66(1), pp.41-46. 
Sherr, E.B. and Sherr, B.F., 2002. Significance of predation by protists in aquatic microbial food webs. Antonie Van Leeuwenhoek, 81(1), pp.293-308.

Sherr, E.B. and Sherr, B.F., 2018. Protistan Grazing Rates via Uptake of Fluorescently Labeied Prey. In Handbook of methods in aquatic microbial ecology (pp. 695-701). CRC Press.

Siano, R., Alves-de-Souza, C., Foulon, E., Bendif, El M. Simon, N., Guillou, L., Not, F., 2011. Distribution and diversity of Amoebophryidae parasites across oligotrophic waters of the Mediterranean Sea. Biogeosciences, 8, pp.267-278.

Skovgaard, A., 2014. Dirty tricks in the plankton: Diversity and role of marine parasitic protists. Acta Protozoologica, 53, pp.51-62.

Stan Development Team., 2020. RStan: the R interface to Stan. R package version 2.21.2

Tang, E.P., 1995. The allometry of algal growth rates. Journal of Plankton Research, 17(6), pp.1325-1335.

Velo-Suárez L., Brosnahan, M. L., Anderson, D. M., and McGillicuddy, D. J. Jr., 2013. A quantitative assessment of the role of the parasite Amoebophrya in the termination of Alexandrium fundyense blooms within a small coastal embayment. PLoS One 8(12), pp.e81150.

Weinbauer, M.G. and Höfle, M.G., 1998. Significance of viral lysis and flagellate grazing as factors controlling bacterioplankton production in a eutrophic lake. Applied and Environmental Microbiology, 64(2), pp.431-438.

Worden, A.Z., Follows, M.J., Giovannoni, S.J., Wilken, S., Zimmerman, A.E. and Keeling, P.J., 2015. Rethinking the marine carbon cycle: factoring in the multifarious lifestyles of microbes. Science, 347(6223).

Yih, W. and Coats, D.W., 2000. Infection of Gymnodinium sanguineum by the dinoflagellate Amoebophrya sp.: effect of nutrient environment on parasite generation time, reproduction, and infectivity. Journal of Eukaryotic Microbiology, 47(5), pp.504-510. 


\section{TABLES}

Table 1. Summary of the data used to calculate per-cell carbon and nitrogen released by $S$. acuminata in culture when infected by the parasite strain A120. Per cell values then used to calculate the quantities of dissolved nutrients released through host cell lysis in Salt Pond.

\begin{tabular}{|l|c|c|c|c|c|}
\hline & \multicolumn{3}{|c|}{ Scrippsiella-A120 Co-culture } & Event 1 & Event 3 \\
\hline Total Infected Cells (per L) & \multicolumn{3}{|c|}{$1,557,195$} & 31,248 & 25,587 \\
\hline & T0 (mg/L) & T85 (mg/L) & Per Cell (ng/L) & $(\mathrm{mg} / \mathrm{L})$ & $(\mathrm{mg} / \mathrm{L})$ \\
\hline Dissolved Organic Carbon & 364.6 & 413.9 & 31.66 & 1.13 & 0.92 \\
\hline Nitrate+Nitrite & 10.045 & 10.119 & 0.05 & 0.0016 & 0.0013 \\
\hline Ammonium & 7.735 & 7.956 & 0.14 & 0.0044 & 0.0036 \\
\hline
\end{tabular}


FIGURES
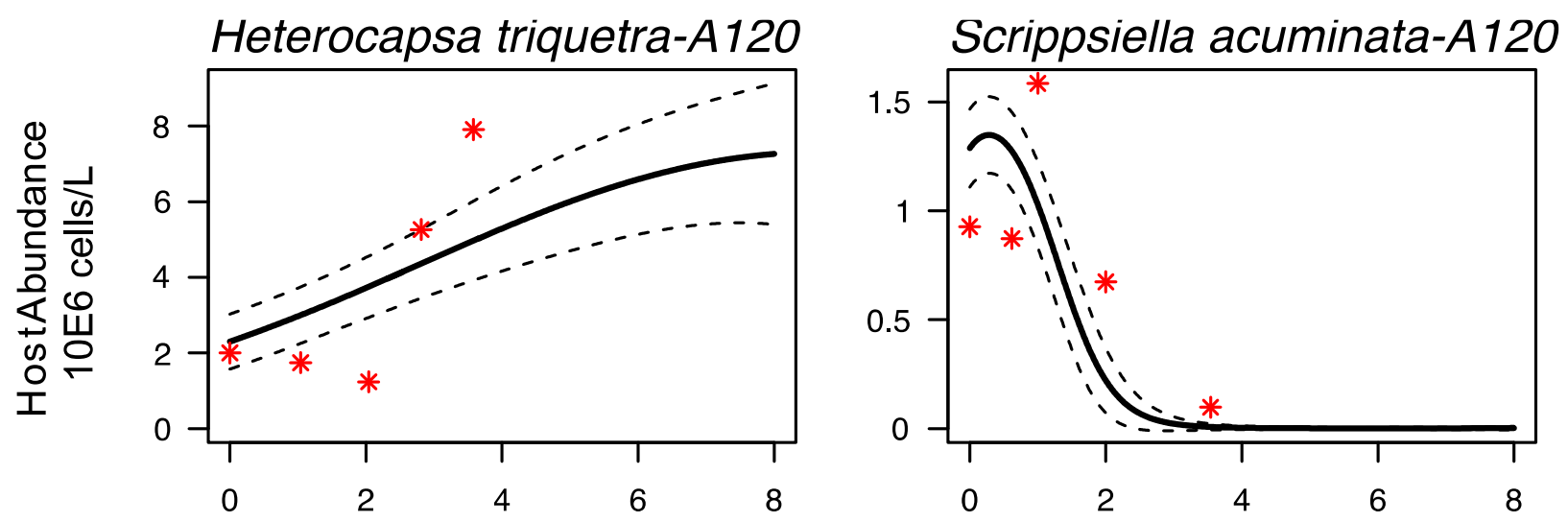

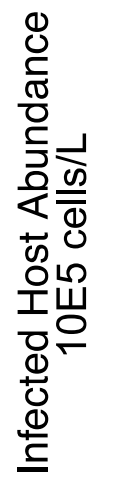
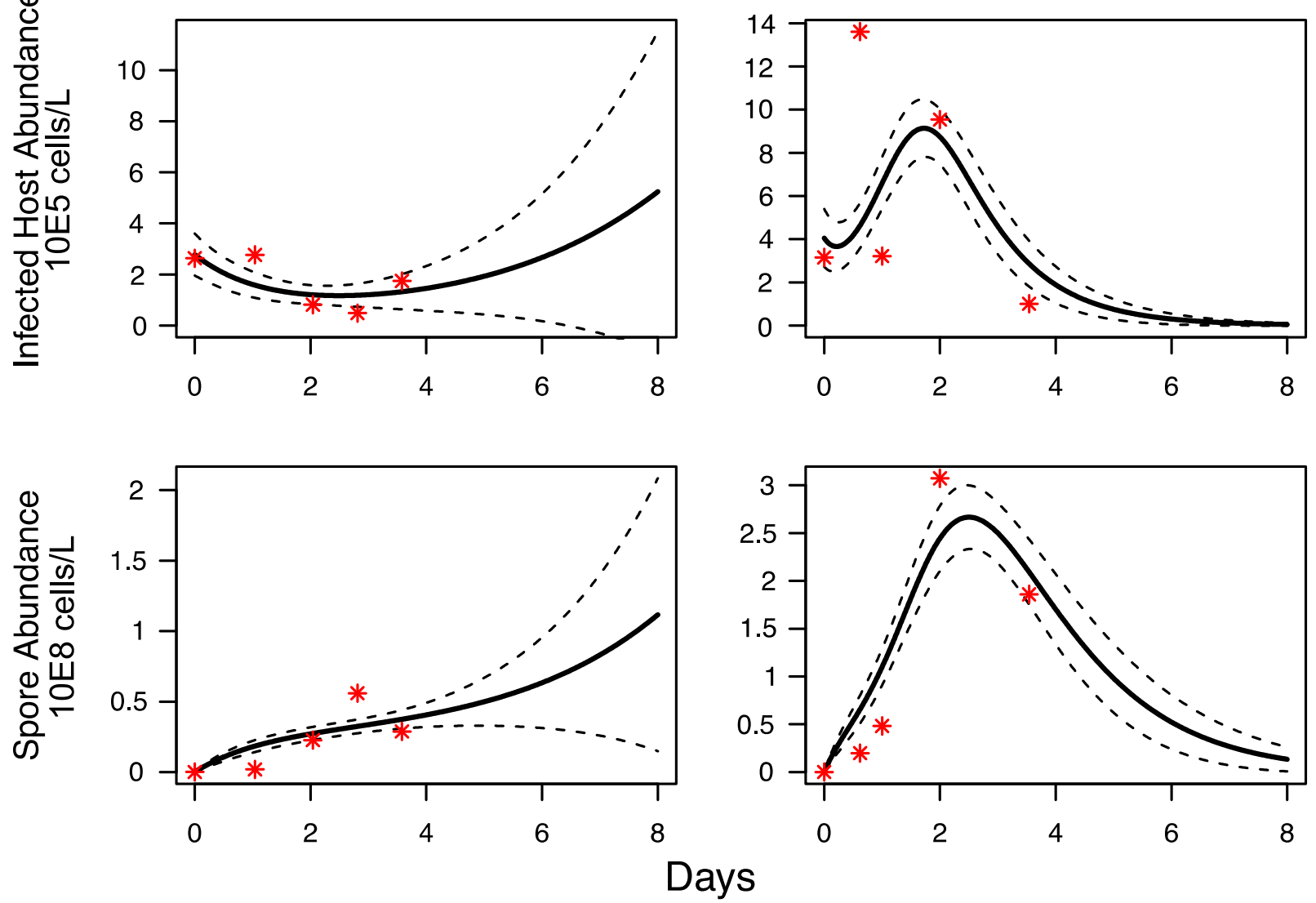

Figure 1. Plots indicate the concentrations of uninfected host, infected host, and free-living parasite cells enumerated by CARD-FISH and epifluorescent microscopy (red marks), and the means for the state variables (across the MCMC iterations; black lines) and 95\% credible intervals (dashed lines) for each of the two culture-based experiments. 

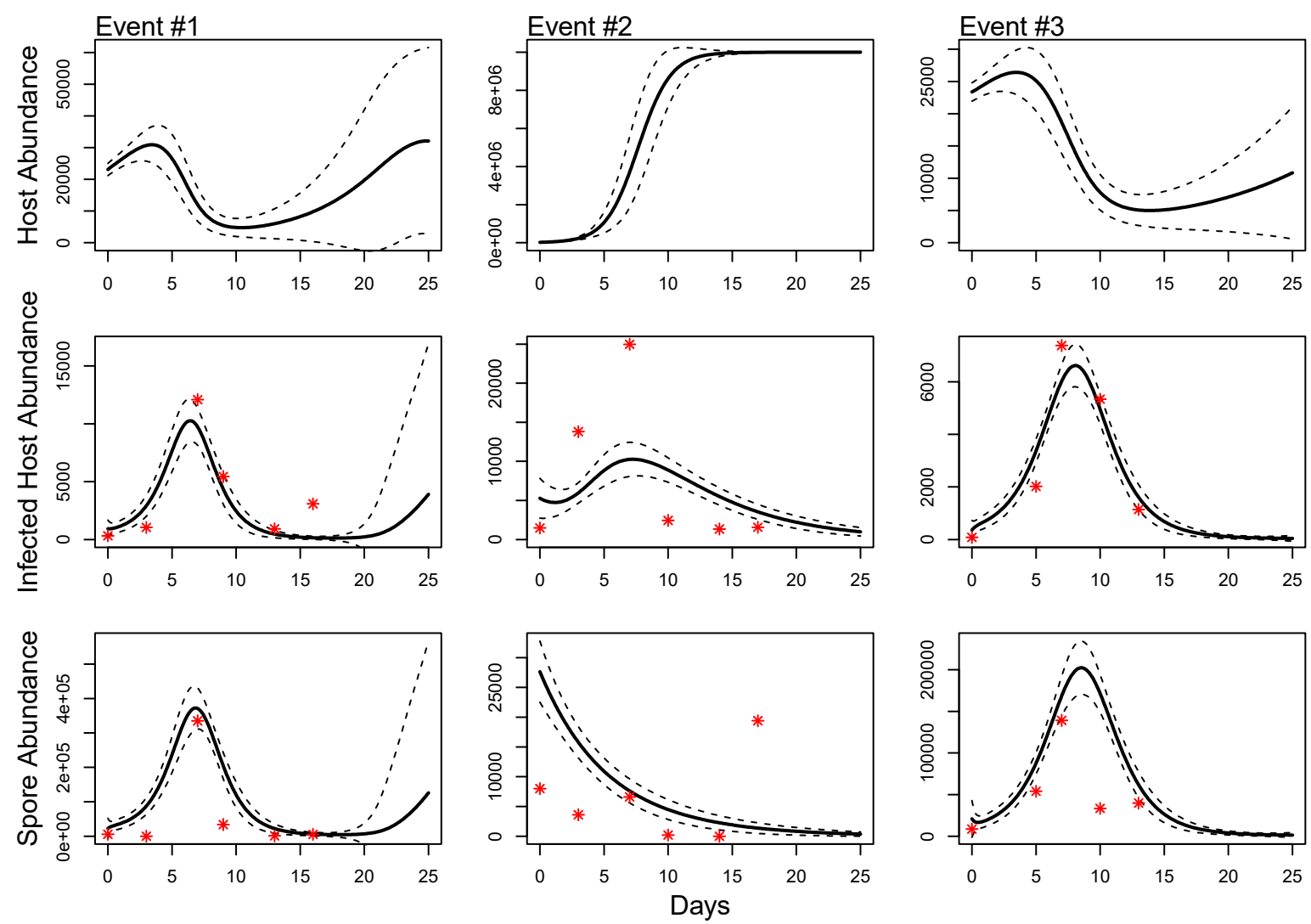

Figure 2. Plots indicate the concentrations of infected hosts and free-living parasite cells enumerated by CARD-FISH and epifluorescent microscopy (red marks) for the three infection events observed in Salt Pond (Event 1-July, Event 2-August, Event 3-September). Uninfected host concentrations were synthetically derived from the model from calculations of the state variables across the time series. Means for the state variables (across the MCMC iterations) and $95 \%$ credible intervals are presented as black and dashed lines, respectively. 
a.

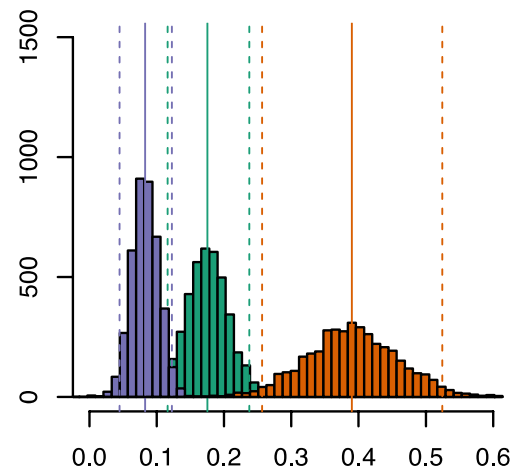

d.

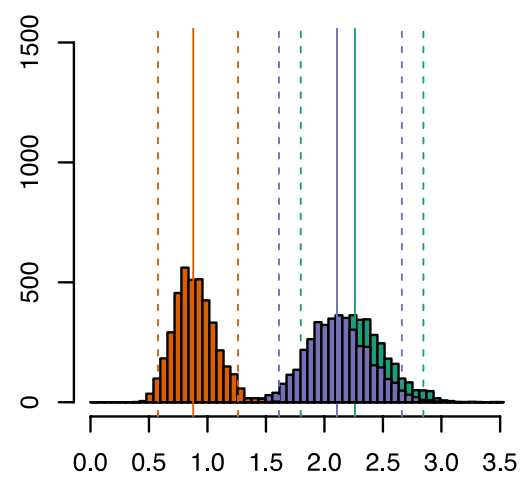

b.

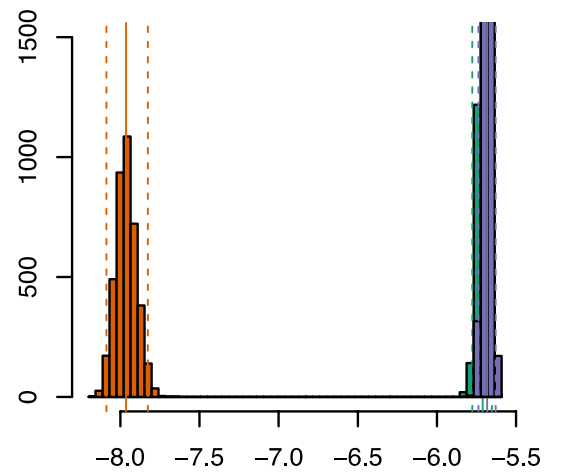

e.

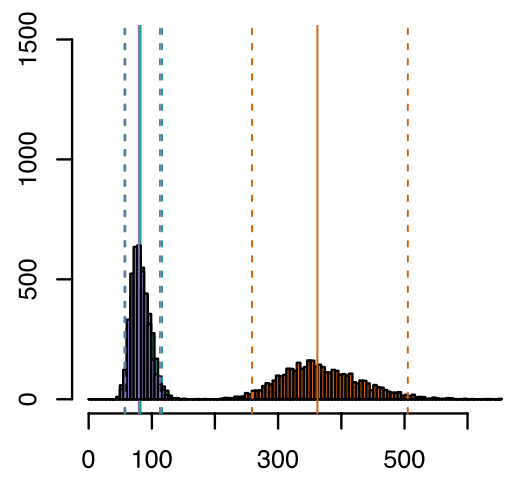

C.

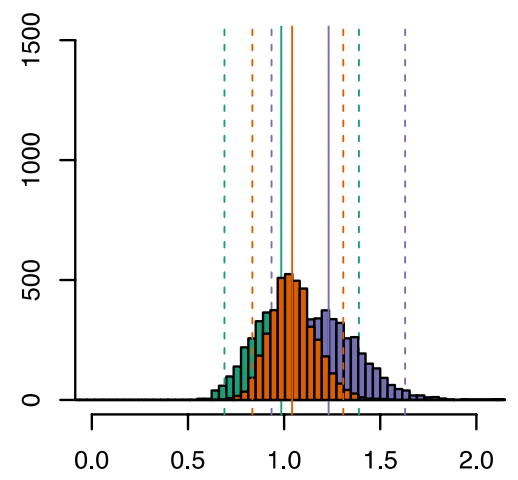

Event\#1

- Event\#3

- S. acuminata-A120

Figure 3. The values of the model parameters for a) host growth rate $r$ (per day), b) infection intensity $a /\left(1+a h_{1}\right)$, c) infection turnover time $h_{2}$ (days), d) parasite mortality $m$ (per day), and e) parasite reproductive output $e$ (spores per infection) were plotted for infection events for which model fits indicated higher degrees of certainty (described by narrower $95 \%$ credible intervals) and in-situ observations that closely matched model fits. The solid lines represent the mean parameter values, with $95 \%$ credible intervals represented by the dashed lines. Narrower distributions of parameter values indicate higher degrees of certainty for the given values. The overlap between parameter distributions indicates the degree of similarity for a given parameter. 


\section{SUPPLEMENTARY FIGURES}
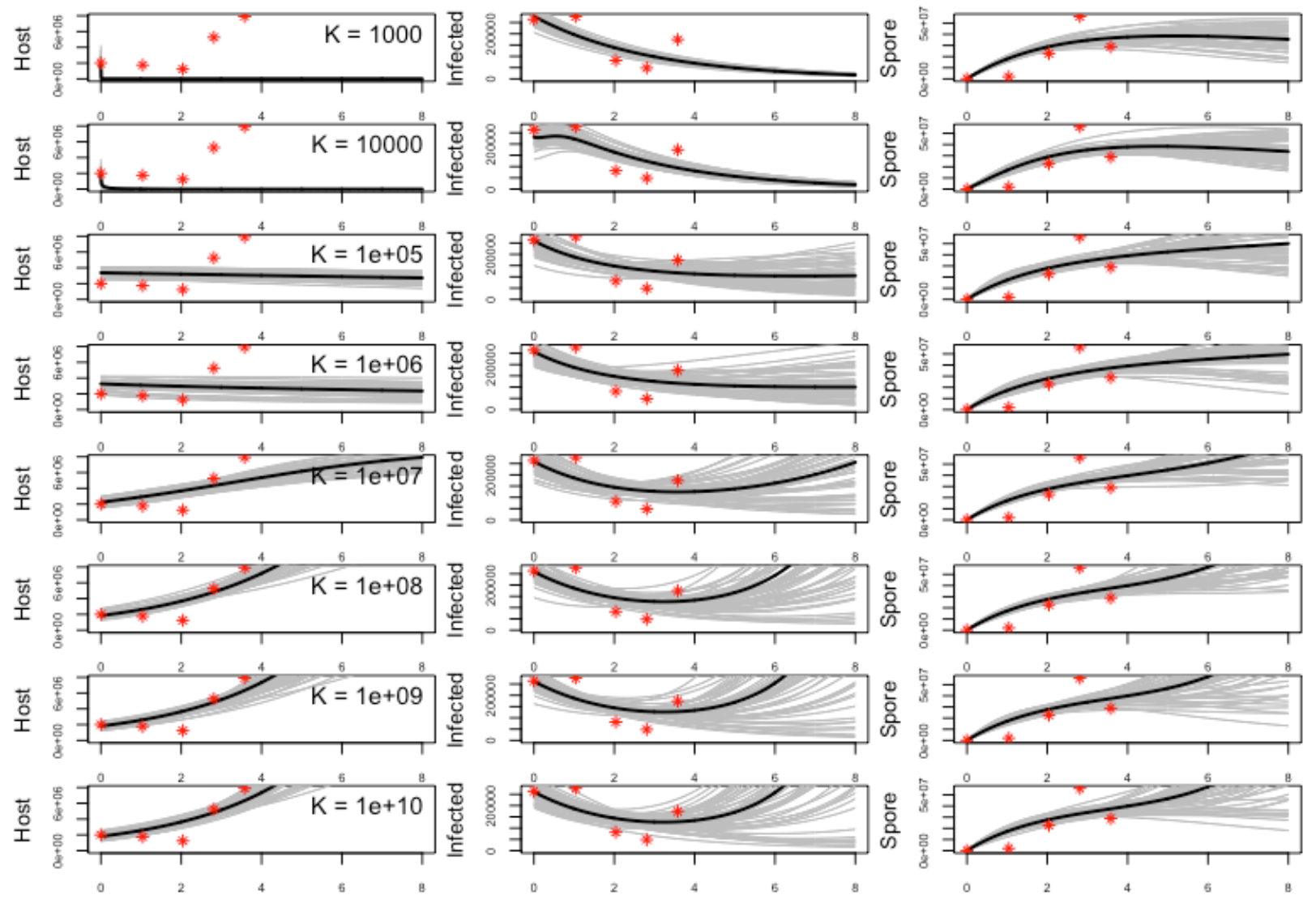

Figure S1. Plots showing the adjustment of the carrying capacity (K) and the changes to the model fits for observations of uninfected hosts, infected hosts, and free-living parasites (red marks) enumerated in the Heterocapsa triquetra-A120 culture experiment. 

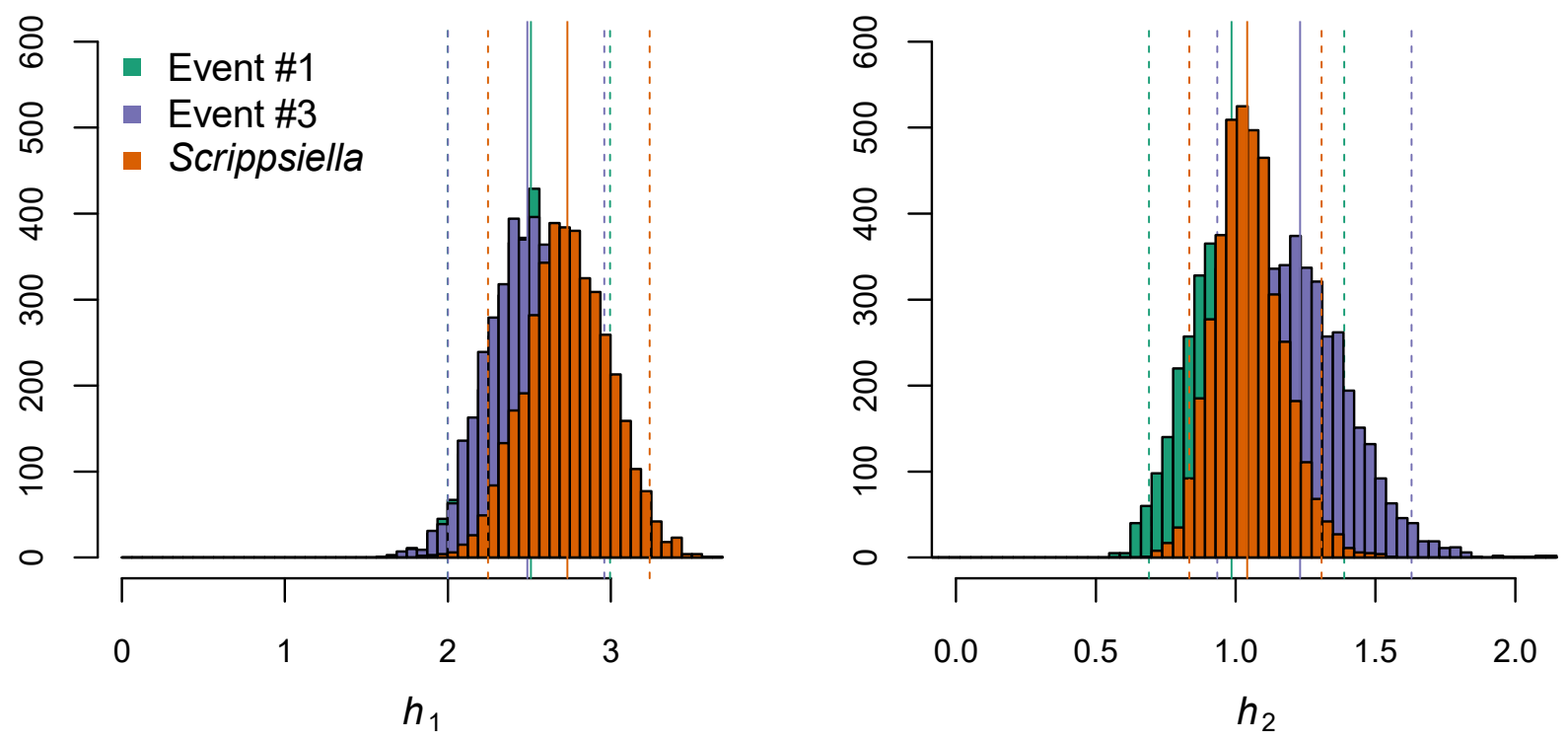

Figure S2. A comparison of the parameter distributions for infection handling time (days), $h_{1}$, and infection turnover time (days), $h_{2}$, plotted for model fits that closely matched in-situ observations. 


\section{REFLECTION}

The inspiration for this thesis began when studying harmful algal blooms in the Nauset Marsh System (Eastham, MA, USA) and potential biological controls on bloom-formation (Choi et al., 2015). In other coastal systems, including the Penzé estuary and the Chesapeake Bay, syndinian parasites infected large fractions of dinoflagellate populations and the peaks of these infections appeared to coincide with the termination of algal blooms (Coats et al., 1996, Chambouvet et al., 2008). Within Nauset Marsh, syndinian parasites were observed infecting both the red tide alga Alexandrium catenella and, in the absence of this host, blooming Heterocapsa triquetra. As I was making these first-hand observations, publications summarizing global ocean surveys were reporting abundant syndinian parasite sequences across diverse marine systems (de Vargas et al., 2015, Pernice et al., 2016), including polar waters (Cleary and Durbin, 2016, Clarke et al., 2019), oxygen minimum zones (Orsi et al., 2012), and oligotrophic seas (Siano et al., 2011). The synthesis of these observations inspired several fundamental research questions:

1. Who are the hosts for many clades of Syndiniales known only by their marker gene sequences?

2. How do seasonal changes to protist community composition impact syndinian parasite prevalence and diversity?

3. How does Syndiniales parasitism impact marine food webs and biogeochemical cycles?

The design of my thesis work incorporated high-resolution field sampling, molecular techniques, epifluorescent microscopy, statistical analyses, culture-based observations, and modeling of hostparasite interactions to address some of the persisting knowledge gaps in syndinian ecology.

Chapter one leveraged spatial and temporal metabarcode sequencing datasets collected at a long-term ecological research site (Saanich Inlet, Vancouver Island, BC) to infer interactions between Syndiniales and putative host taxa. Saanich Inlet is a seasonally-stratified coastal fjord where oxygen is depleted in the summer months. Oxygen is resupplied to deeper waters during a fall renewal period. Previous studies noted the abundance of syndinian parasite sequences in marker gene datasets throughout the year (Orsi et al., 2012), suggesting a significant role for these taxa in microbial communities. I examined how the annual stratification cycle in Saanich Inlet structured the protist community along the redoxcline and explored potential relationships 
between protist and metazoan populations and syndinian parasites using co-occurrence analyses. Notably, syndinian parasite sequences were found to be significantly correlated with the marine haptophyte, Phaeocystis antarctica. This co-occurrence pattern was echoed by studies in the English Channel (Monchy et al., 2012, Christaki et al., 2017); however, no direct observations of Phaeocystis infected by Syndiniales parasites have been made to date. Significant correlations between syndinian parasites and tintinnid ciliates and metazoa presented new questions about the roles of parasites in marine food webs. Ciliates and metazoa have been reported hosting some species of syndinian parasites (Skovgaard et al., 2005, Guillou et al., 2008, Bachvaroff et al., 2012, Zamora-Terol et al., 2021), but may also graze on parasite spores released from other infections. This study would have been improved by including sampling for microscopy so that parasites and their spores could have been visualized and I could have confirmed active syndinian infections. It also would have been improved by increasing sampling frequencies to better capture co-occurrence patterns of Syndiniales and bloom-forming taxa that may only be abundant in the water column for a few weeks.

The conclusions reached in chapter one helped guide a sampling regime at a field site proximal to Woods Hole Oceanographic Institution. Chapter two describes a high-resolution time series conducted in Salt Pond (Falmouth, MA, USA), a seasonally-stratified coastal tidal pond where syndinian parasites were first documented infecting multiple host morphotypes in 2017. Sampling was conducted every two to three days from March-October 2018 at three depths representing oxic, oxycline, and anoxic water column conditions during peak stratification. Microscopy data using the Group II-specific CARD-FISH probe and iTag barcode sequencing libraries determined infections were infrequent during the spring bloom (MarchMay). The protist community was dominated by primary producers, mainly diatoms and chlorophytes, that were not infected by Group II Syndiniales. Intensifying stratification appeared to promote dinoflagellate growth, which corresponded to Group II Syndiniales infection events in July, August, and September. The sequencing libraries identified different Group II Syndiniales clades present during each of the three infection events and microscopy revealed a variety of host morphologies. Unfortunately, direct host-parasite interactions could not be confirmed with these datasets. In the Nauset Marsh system, the red-tide-forming dinoflagellate Alexandrium catenella bloom concentrations reached $10^{6}$ cells/L and up to $30 \%$ of cells were infected (Velo-Suarez et al., 2013). By contrast, some host populations in Salt Pond were less 
abundant, but significantly impacted by infections. For example, the concentration of scrippsielloid cells observed on July $3^{\text {rd }}$ was approximately 16,000 cells/L (9.6\% of protists); however, $16 \%$ of cells with this morphology were infected by Group II Syndiniales. The methods I applied in Salt Pond highlighted the importance of visualizing biological interactions in conjunction with molecular analyses to learn about the seasonal diversity and infection dynamics of syndinian parasites; however, these methodologies do not provide evidence of specific host-parasite interactions, as morphologies of infected hosts observed on filters prepared for fluorescence in-situ hybridization are insufficient to make precise taxonomic identifications. Hosts of specific parasite clades need to be confirmed through culture-based analyses, single-cell sequencing of infected cells, or the development of species-specific probes.

In the third chapter, I aimed to test a previously-published model of host-parasite interactions with Bayesian approaches using new culture-based experiments and field observations. The outputs of the model and nutrient analyses from the laboratory experiments were then used to estimate the contributions of carbon and nitrogen from host cell lysis to pools of dissolved organic matter during individual infection events reported in Salt Pond. Equations first proposed by Salomon and Stolte (2010) were used as the framework to model host-parasite interactions both in culture and in the field. Culture-based experiments used two common coastal dinoflagellate taxa, Heterocapsa triquetra and Scrippsiella acuminata, and a Group II Clade 2 parasite (A120) compatible with both hosts. Observations of infected hosts and free-living parasite spores during the three infection events in Salt Pond (described in chapter two) were also used to test the model.

We found the equations of the model could be fitted to culture experiments and field observations that showed boom-bust infection patterns (S. acuminata-A120, Event 1 in July, and Event 3 in September). The H. triquetra culture was less susceptible to A120 and the host population continued to grow exponentially as the parasite population declined. This pattern was not successfully modeled by the Salomon and Stolte equations. Creating and testing an additional parameter to add to the model that describe parasite virulence may aid in modeling infection scenarios where the host outcompetes the parasite. Infection event 2, which occurred in Salt Pond in August 2018, could similarly not be modeled using the Salomon and Stolte equations. During this event, high concentrations of diverse host morphotypes infected by Group II Syndiniales parasites were recorded, but free-living spores were not abundant in samples 
collected at the same time points. Adjusting the parasite mortality term did not improve the model fit. Grazing, a component of all microbial food webs, may have exerted significant topdown pressure on parasite populations during event 2; therefore, incorporating additional grazing terms, as tested in Alves-de-Souza et al. (2015), may improve future iterations of the Salomon and Stolte model.

The total number of cells infected in the laboratory-based S. acuminata-A120 culture experiment was estimated using the model equations. Nutrient analyses from the initial time point and the termination of the experiment after four days provided information on the dissolved nutrients in the culture media that were likely released through host cell lysis. S. acuminata represented only one of several host morphotypes observed in Salt Pond; however, these "per cell" dissolved organic carbon, ammonium, nitrate and nitrite concentrations were used to provide a rough estimate of the nutrients released during the infection events in July and September 2018 in Salt Pond. The findings of chapter three highlighted the successful application of the Salomon and Stolte equations on datasets from culture-based experiments and observations in the field where host and parasite populations demonstrate peaks and crashes as a result of parasitic interactions. However, future research is needed to update the model equations to incorporate variations in host susceptibility/parasite virulence as well as external trophic pressures. It also would be wise to expand the range of culture experiments to quantify the nutrient fluxes associated with additional host and parasite interactions. Then, I believe we can develop more complex models to quantify the biogeochemical impacts of syndinian parasitism across the global ocean because the model will accommodate host-parasite interactions that show outcomes different from the boom-bust infection patterns.

The most exciting area for continued research is the realm of single-cell -omics. Single cell genomics can provide more definitive evidence of specific host-parasite interactions. However, this area of research requires targeted approaches to isolate cells of interest and may also benefit from advances in co-culturing hosts and parasites. During this thesis, I attempted to sequence and assemble the genomes of previously uncultured Syndiniales parasites by isolating infected host cells collected from Salt Pond in 2019. The pond was sampled daily for 40 days beginning on July 1, 2019 in an effort to capture another infection event similar to those observed during July, August, and September 2018. Approximately one liter of seawater was collected at one meter depth twice per week and placed on ice for transport to the laboratory. The 
water was filtered through a 5 um Nitex mesh to collect protist cells. Active syndinian parasites have a green autofluorescence that can be used to identify infections; therefore, a FACSCaliber (Becton-Dickinson) flow cytometer was used to sort based on fluorescence and concentrate potentially infected live cells. The concentrated samples after sorting were fixed with the cryopreservative glyTE (glycerol-Tris-EDTA) and stored at -80C.

An infection event comparable to 2018 was not observed in 2019. Instead, a Raphidophyte, which was not infected by syndinian parasites, bloomed in the pond. From the available samples, I determined the cells collected on July $26^{\text {th }}$ had the highest concentration of syndinian infections ( $\sim 1 \%$ of all protists, determined by CARD-FISH) and I manually isolated cells from the corresponding glyTE-preserved sample. Individual cells were washed in phosphate buffered saline, then placed in individual wells and stored at -80C. DNA extractions and whole genome amplifications were performed following the protocol detailed by Stepanauskas et al. (2017), but optimized the amplification time to 5.5 hours based on DNA quantification. Amplification durations have differed between studies of single cell protist genomes (Seeleuthner et al., 2018, Sieracki et al., 2019), suggesting this step may need to be adjusted to target the taxa of interest. The amplified genome products were then screened using a universal V4 primer set, which targets a hypervariable region of the $18 \mathrm{~S}$ gene in eukaryotes. Samples that amplified a product approximately 400 base pairs in length, suggesting the presence of amplifiable eukaryotic DNA, were selected for whole genome sequencing. The abbreviated methods detailed above reflect available equipment and laboratory resources; however, deviations from the sample preparation methods outlined in Stepanauskas et al. (2017) may have introduced contaminants or biased non-target taxa in amplification and sequencing steps.

The sequences of 28 isolated cells were assembled using Spades, idba, and Megahit. Total assembly sizes averaged approximately $30 \mathrm{Mbp}$. The total genome size of a Group II-Clade 2 Syndiniales is estimated to be $100 \mathrm{Mbp}$; therefore, I aimed to identify multiple samples containing closely-related Syndiniales $18 \mathrm{~S}$ marker gene sequences to co-assemble and generate a more complete Syndiniales genome. The estimated completeness of each sample was calculated with BUSCO (Benchmarking Universal Single Copy Orthologs) using the Alveolate database to target samples containing orthologous genes most similar to Syndiniales (Superphylum Alveolata). The sample with the highest estimated completeness was 29\%; however, most samples were only approximately $12 \%$ complete. In spite of the rigorous screening efforts, most 
of the raw sequence reads and assembled contigs were annotated to bacterial and viral sequences when compared to sequences in the NCBI Blast database and the MMETSP (Marine Microbial Eukaryote Transcriptome Sequencing Project). I compared the sequences from the Salt Pond cells to published Syndiniales genomes and found ten sequence reads (out of millions) mapped to the draft genome. Furthermore, a phylogenetic tree constructed from orthologous genes could not be constructed because too few genes were shared between the Salt Pond samples and reference taxa (8 genes out of 177). Based on these outcomes, I was unable to construct a syndinian genome from single-cell isolates collected in Salt Pond, or to make a firm taxonomic identificaiton of an infected host and its parasite.

There have been few studies that have fully reconstructed genomes of marine eukaryotes from environmental samples, underscoring the need to develop techniques that can be applied to study the uncultured majority of protists. The techniques used to PCR amplify and sequence samples isolated from Salt Pond may have biased bacterial contamination that originated in the laboratory or from DNA contained within food vacuoles of heterotrophic dinoflagellates or in the external microbiome of the cells. Alternatively, the databases used to annotate the contigs generated by the sequence assemblies may not contain closely-related taxa, producing misleading taxonomic assignments. To date, there are only twelve dinoflagellate genomes published in genetic repositories, representing $0.02 \%$ of the NCBI Genomes database. The first Group II Syndiniales genome was assembled by sequencing a cultured isolate maintained by the Roscoff culture collection (John et al., 2019). A subsequent study compared the genomes of three Amoebophrya (Group II) cultures and found 36-47\% of predicted proteins were shared between strains. The orthologs shared $48-51 \%$ of amino acid sequence similarity, which was comparable to the levels of similarity between Amoebophrya and the more distantly related perkinsid, Perkinsus marinus, and apicomplexan Plasmodium falciparum (Farhat et al., 2021). Efforts to assemble syndinian genomes from other marine systems (i.e. Tara Oceans) with single-cells and metagenome-assembled genomes have not yielded additional sequences (Seeleuthner et al., 2018, Alexander et al., 2021).

There are a number of rapidly-evolving fields in science and engineering that may one day facilitate the reconstruction of syndinian genomes from across the global ocean, unlocking opportunities to quickly establish host-parasite interactions and dive into the molecular mechanisms that underpin syndinian parasitism. Microfluidic techniques that isolate small cells 
in bubbles containing reagents for RNA or DNA amplification may increase sample throughput (Mazutis et al., 2013, Zilionis et al., 2017). Given the unknown prevalence of syndinian parasites at the time of sampling an environment, that higher throughput approach could increase the odds of detecting and successfully amplifying target taxa. Sequencing technologies are also evolving to include long-read approaches that may benefit the assembly of genomes with repetitive elements (Farhat et al., 2021). Dinoflagellate, and potentially Syndiniales, genomes contain these sections of non-coding DNA that are often difficult to assemble with short read sequences. Last, the software and databases used to assemble and annotate marine eukaryotes are ever-changing as new information is added. Cumulatively, these tools and techniques stand to change how we gather and interpret marine microbial datasets, and I look forward to revisiting the field of syndinian genomics in my future career. 


\section{REFERENCES}

Alexander, H., Hu, S.K., Krinos, A.I., Pachiadaki, M., Tully, B.J., Neely, C.J. and Reiter, T., 2021. Eukaryotic genomes from a global metagenomic dataset illuminate trophic modes and biogeography of ocean plankton. bioRxiv.

Alves-de-Souza, C., Pecqueur, D., Le Floc'h, E., Mas, S., Roques, C., Mostajir, B., Vidussi, F., Velo-Suares, L., Sourisseau, M., Fouilland, E., Guillou, L., 2015. Significance of plankton community structure and nutrient availability for the control of dinoflagellate blooms by parasites: a modeling approach. PLoS One. 2015; 10(6): e0127623.

Bachvaroff, T.R., Kim, S., Guillou, L., Delwiche, C.F. and Coats, D.W., 2012. Molecular diversity of the syndinean genus Euduboscquella based on single-cell PCR analysis. Applied and Environmental Microbiology, 78(2), pp.334-345.

Chambouvet, A., Morin, P., Marie, D. and Guillou, L., 2008. Control of toxic marine dinoflagellate blooms by serial parasitic killers. Science, 322(5905), pp.1254-1257.

Choi, C.J., Brosnahan, M.L., Sehein, T.R., Anderson, D.M. and Erdner, D.L., 2017. Insights into the loss factors of phytoplankton blooms: The role of cell mortality in the decline of two inshore Alexandrium blooms. Limnology and Oceanography, 62(4), pp.1742-1753.

Christaki, U., Genitsaris, S., Monchy, S., Li, L.L., Rachik, S., Breton, E. and Sime-Ngando, T., 2017. Parasitic eukaryotes in a meso-eutrophic coastal system with marked Phaeocystis globosa blooms. Frontiers in Marine Science, 4, p.416.

Clarke, L.J., Bestley, S., Bissett, A. and Deagle, B.E., 2019. A globally distributed Syndiniales parasite dominates the Southern Ocean micro-eukaryote community near the sea-ice edge. The ISME Journal, 13(3), pp.734-737.

Cleary, A.C. and Durbin, E.G., 2016. Unexpected prevalence of parasite 18S rDNA sequences in winter among Antarctic marine protists. Journal of Plankton Research, 38(3), pp.401-417.

Coats, D.W., Adam, E.J., Gallegos, C.L. and Hedrick, S., 1996. Parasitism of photosynthetic dinoflagellates in a shallow subestuary of Chesapeake Bay, USA. Aquatic Microbial Ecology, 11(1), pp.1-9.

De Vargas, C., Audic, S., Henry, N., Decelle, J., Mahé, F., Logares, R., Lara, E., Berney, C., Le Bescot, N., Probert, I. and Carmichael, M., 2015. Eukaryotic plankton diversity in the sunlit ocean. Science, 348(6237).

Farhat, S., Le, P., Kayal, E., Noel, B., Bigeard, E., Corre, E., Maumus, F., Florent, I., Alberti, A., Aury, J.M. and Barbeyron, T., 2021. Rapid protein evolution, organellar reductions, and invasive intronic elements in the marine aerobic parasite dinoflagellate Amoebophrya spp. BMC Biology, 19(1), pp.1-21. 
Guillou, L., Viprey, M., Chambouvet, A., Welsh, R.M., Kirkham, A.R., Massana, R., Scanlan, D.J. and Worden, A.Z., 2008. Widespread occurrence and genetic diversity of marine parasitoids belonging to Syndiniales (Alveolata). Environmental Microbiology, 10(12), pp.3349-3365.

John, U., Lu, Y., Wohlrab, S., Groth, M., Janouškovec, J., Kohli, G.S., Mark, F.C., Bickmeyer, U., Farhat, S., Felder, M. and Frickenhaus, S., 2019. An aerobic eukaryotic parasite with functional mitochondria that likely lacks a mitochondrial genome. Science Advances, 5(4), p.eaav1110.

Mazutis, L., Gilbert, J., Ung, W.L., Weitz, D.A., Griffiths, A.D. and Heyman, J.A., 2013. Singlecell analysis and sorting using droplet-based microfluidics. Nature Protocols, 8(5), pp.870-891.

Monchy, S., Grattepanche, J.D., Breton, E., Meloni, D., Sanciu, G., Chabé, M., Delhaes, L., Viscogliosi, E., Sime-Ngando, T. and Christaki, U., 2012. Microplanktonic community structure in a coastal system relative to a Phaeocystis bloom inferred from morphological and tag pyrosequencing methods. PLoS One, 7(6), p.e39924.

Orsi, W., Song, Y.C., Hallam, S. and Edgcomb, V., 2012. Effect of oxygen minimum zone formation on communities of marine protists. The ISME journal, 6(8), pp.1586-1601.

Pernice, M.C., Giner, C.R., Logares, R., Perera-Bel, J., Acinas, S.G., Duarte, C.M., Gasol, J.M. and Massana, R., 2016. Large variability of bathypelagic microbial eukaryotic communities across the world's oceans. The ISME Journal, 10(4), pp.945-958.

Salomon, P.S. and Stolte, W., 2010. Predicting the population dynamics in Amoebophrya parasitoids and their dinoflagellate hosts using a mathematical model. Marine Ecology Progress Series, 419, pp.1-10.

Seeleuthner, Y., Mondy, S., Lombard, V., Carradec, Q., Pelletier, E., Wessner, M., Leconte, J., Mangot, J.F., Poulain, J., Labadie, K. and Logares, R., 2018. Single-cell genomics of multiple uncultured stramenopiles reveals underestimated functional diversity across oceans. Nature Communications, 9(1), pp.1-10.

Siano, R., Alves-de-Souza, C., Foulon, E., Bendif, E.M., Simon, N., Guillou, L. and Not, F., 2011. Distribution and host diversity of Amoebophryidae parasites across oligotrophic waters of the Mediterranean Sea. Biogeosciences, 8(2), pp.267-278.

Sieracki, M.E., Poulton, N.J., Jaillon, O., Wincker, P., de Vargas, C., Rubinat-Ripoll, L., Stepanauskas, R., Logares, R. and Massana, R., 2019. Single cell genomics yields a wide diversity of small planktonic protists across major ocean ecosystems. Scientific reports, 9(1), pp.1-11.

Skovgaard, A., Massana, R., Balague, V. and Saiz, E., 2005. Phylogenetic position of the copepod-infesting parasite Syndinium turbo (Dinoflagellata, Syndinea). Protist, 156(4), pp.413423. 
Stepanauskas, R., Fergusson, E.A., Brown, J., Poulton, N.J., Tupper, B., Labonté, J.M., Becraft, E.D., Brown, J.M., Pachiadaki, M.G., Povilaitis, T. and Thompson, B.P., 2017. Improved genome recovery and integrated cell-size analyses of individual uncultured microbial cells and viral particles. Nature Communications, 8(1), pp.1-10.

Velo-Suárez, L., Brosnahan, M.L., Anderson, D.M. and McGillicuddy Jr, D.J., 2013. A quantitative assessment of the role of the parasite Amoebophrya in the termination of Alexandrium fundyense blooms within a small coastal embayment. PLoS One, 8(12), p.e81150.

Zamora-Terol, S., Novotny, A. and Winder, M., 2021. Molecular evidence of host-parasite interactions between zooplankton and Syndiniales. Aquatic Ecology, 55(1), pp.125-134.

Zilionis, R., Nainys, J., Veres, A., Savova, V., Zemmour, D., Klein, A.M. and Mazutis, L., 2017. Single-cell barcoding and sequencing using droplet microfluidics. Nature Protocols, 12(1), pp.44-73. 